\title{
SEARCH FOR LIGHT DARK MATTER PRODUCED IN A PROTON BEAM DUMP
}

\author{
Remington Tyler Thornton
}

Submitted to the faculty of the University Graduate School in partial fulfillment of the requirements

for the degree

Doctor of Philosophy in the Department of Physics,

Indiana University

February 2017 
Accepted by the Graduate Faculty, Indiana University, in partial fulfillment of the requirements for the degree of Doctor of Philosophy.

\section{Doctoral Committee}

Rex Tayloe, Ph.D. (Chair)

Charles Horowitz, Ph.D.

James Musser, Ph.D.

W. Michael Snow, Ph.D.

February 6, 2017 
Copyright (C) 2017

Remington Tyler Thornton 


\section{ACKNOWLEDGMENTS}

This work would not have been completed without the help and support of several people. My wife, Holly, has been supportive throughout the entire process, while my children, David and Samuel, have been a huge motivation, always ready to entertain me during much needed breaks. The teaching and encouragement I received while under the leadership of my professors at Abilene Christian University formed the bedrock of my success in graduate school. Thank you.

I am thankful Rex Tayloe took a chance on me: helped me get into Indiana University, was willing to be my advisor, and allowed my work schedule to be flexible once my children were born. Rex, Robert Cooper, and Lance Garrison helped me get my feet wet at IU and tought me how to work in/with a small collaboration. Rex, Robert, and Richard Van de Water encouraged me to make this analysis my own, not adhearing to what others had invision the analysis would be. I am thankful for their guidence and many productive conversations.

I am thankful for Ranjan Dharmapalan's hard work in getting this analysis started, as an extension to his thesis, and helping me get up to speed. Much apparition goes out to Zarko Pavlovic in his continue work on the MiniBooNE computers. None of the current MiniBooNE analyses can be done without his hard work. This analysis is an accumulation of over a decade of work by a large number of people: the MiniBooNE collaboration, Fermilab Accelerator Division, and the neutrino group at Los Alamos to name a few. Their work in building/understanding the Booster Neutrino Beamline and MiniBooNE detector for the oscillation and cross section analyses allowed this analysis to be completed. I am thankful for collaborators like Byron Roe, Bill Louis, and Mike Shaevitz who still contribute and phone-in to detailed analyses meetings.

This work could not have been completed with out the efforts of Patrick deNiverville 
and Brian Batell. I am so thankful for their work in developing the dark matter generator and being willing to answer my questions. 


\section{Remington Tyler Thornton}

\section{SEARCH FOR LIGHT DARK MATTER PRODUCED IN A PROTON BEAM DUMP}

Cosmological observations indicate that our universe contains dark matter (DM), yet we have no measurements of its microscopic properties. Whereas the gravitational interaction of DM is well understood, its interaction with the Standard Model is not. Direct detection experiments, the current standard, search for a nuclear recoil interaction and have a low-mass sensitivity edge of order $1 \mathrm{GeV}$. A path to detect DM with mass below $1 \mathrm{GeV}$ is the use of accelerators producing boosted low-mass DM. Using neutrino detectors to search for low-mass DM is logical due to the similarity of the DM and neutrino signatures in the detector. The MiniBooNE experiment, located at Fermilab on the Booster Neutrino Beamline, has produced the first proton beam-dump light DM search results. Using dark matter scattering from nucleons $90 \%$ confidence limits were set over a large parameter space and, to allow tests of other theories, a model independent DM rate was extracted.

Rex Tayloe, Ph.D. (Chair)

Charles Horowitz, Ph.D.

James Musser, Ph.D.

W. Michael Snow, Ph.D. 


\section{CONTENTS}

List of Abbreviations $\quad \mathrm{x}$

List of Symbols $\quad$ x

List of Appendices $\quad$ xi

List of Figures $\quad$ xii

List of Tables $\quad$ xxi

1 Introduction 1

2 Dark Matter Theory 6

2.1 Order of Magnitude Calculation . . . . . . . . . . . . . . . 6

2.2 Candidate Dark Matter Models . . . . . . . . . . . . . . . . 8

2.3 Light Dark Matter Theory . . . . . . . . . . . . . . . . . . . . 9 9

2.3.1 Muon Magnetic Moment . . . . . . . . . . . . . . . . . . . 11

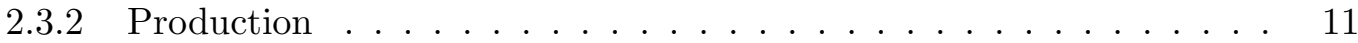

2.3 .3 Interaction . . . . . . . . . . . . . . . . . . . . . . . 13

2.4 Experimental Search . . . . . . . . . . . . . . . . . . . . 14

2.4.1 Dark Matter Direct Detectors . . . . . . . . . . . . . . . . . 14

2.4.2 Previous Beam Dump Experiments . . . . . . . . . . . . . 15

3 Experimental Setup $\quad 19$

3.1 Booster Neutrino Beamline _. . . . . . . . . . . . . . . . . . . . 19

3.2 MiniBooNE Detector . . . . . . . . . . . . . . . . . . . . 25

3.2 .1 DAQ and Trigger System . . . . . . . . . . . . 26

3.2.2 Measuring Beam Unrelated Backgrounds . . . . . . . . . . . . . . . 28 
3.2 .3 Detector Simulation . . . . . . . . . . . . . . . 30

3.2.4 Event Reconstruction _. . . . . . . . . . . . . . . . 33

4 Data Set Distributions $\quad 37$

4.1 Event Selection Cuts . . . . . . . . . . . . . . . . . . . . . 38

4.1.1 NCE Selection Criteria . . . . . . . . . . . . . . . 39

4.1.2 CCQE Selection Events . . . . . . . . . . . . . . . 44

4.1 .3 Definition of $Q_{Q E}^{2} \quad \ldots \ldots \ldots \ldots \ldots \ldots$

4.2 Off-Target Neutrino Simulation . . . . . . . . . . . . . . . . 46

4.2 .1 Simulated Geometry . . . . . . . . . . . . . . . . . 47

4.2 .2 Determining Central-Value Flux _ . . . . . . . . . . . . 50

4.2 .3 Testing CCQE $E_{\nu}^{\mathrm{QE}}$ Predictability $\ldots \ldots \ldots \ldots \ldots$. . . . . . . 61

4.3 Systematic Uncertainty . . . . . . . . . . . . . . . . . 63

4.3 .1 Off-Target Systematics . . . . . . . . . . . . . . . . . . 65

4.4 Final $Q_{Q E}^{2}$ Distributions . . . . . . . . . . . . . . . . 69

$\begin{array}{lll}5 & \text { Fit Procedure } & 73\end{array}$

5.1 Generating Fake Data . . . . . . . . . . . . . . . . 73

5.2 Background-Only Fit . . . . . . . . . . . . . . . . . 75

5.2 .1 Probability of Fit . . . . . . . . . . . . 80

5.2 .2 Propagating Nuisance Uncertainties . . . . . . . . . . . . . 83

5.3 Model Independent Fit . . . . . . . . . . . . . . . . . . . 86

5.3.1 Creating Efficiency Matrix . . . . . . . . . . . . . 86

5.3.2 Generating Predicted $Q_{Q E}^{2}$ Distribution . . . . . . . . . . 90

6 Generating Confidence Limits $\quad 94$

6.1 Light Dark Matter Simulation . . . . . . . . . . . . . . . . . . 94

6.2 Confidence Limits . . . . . . . . . . . . . . . . . . . . . 101 
6.2.1 Traditional $\chi^{2}$ Method . . . . . . . . . . . . . . . . . . 101

6.2 .2 Frequentist Method . . . . . . . . . . . . . . . . 103

7 Conclusion

Bibliography

119

Curriculum Vitae 


\section{LIST OF ABBREVIATIONS}

$\Lambda \mathrm{CDM}$ Lambda-Cold Dark Matter

BNB Booster Neutrino Beamline

BPM beam position monitor

BRB beam-related background

BUB beam-unrelated background

CCQE charged-current quasielastic

CDF cumulative distribution function

CDM cold dark matter

CI confidence interval

CL confidence limit

CMB cosmic microwave background

DAQ data acquisition

detMC detector simulation

dirtMC dirt simulation

DM dark matter

FNAL Fermi National Accelerator Laboratory

IFDB Intensity Frontier Database

JLab Jefferson National Laboratory

LDM light dark matter

LHC Large Hadron Collider

LM loss monitor

MC simulation

MIF model independent fit

MW multiwire

NCE neutral-current elastic

PDF probability distribution function

PMT photomultiplier tube

POT protons on target

ROI region of interest 
SBND Short Baseline Near Detector

SM standard model

SVD singular value decomposition

SW Sanford-Wang distribution

WIMP weakly interacting massive particle 


\section{LIST OF SYMBOLS}

$\alpha^{\prime} \quad$ The dark sector gauge coupling squared divided by $4 \pi$

$\mathrm{CCQE}_{\mathrm{Off}} \quad$ The CCQE sample from the off-target data set

$\mathrm{CCQE}_{\nu} \quad$ The CCQE sample from the $\nu$-Mode data set

$\chi$

Dark matter particle where the mass is given by $m_{\chi}$

$E_{b}$

Binding energy of the nucleon

$E_{\nu}$ or $E_{\nu}^{\text {true }} \quad$ The true neutrino energy

$E_{\nu}^{\mathrm{QE}}$

The reconstructed neutrino energy based off the quasi-elastic assumption

$\epsilon$

The kinetic mixing angle between the dark and light sector

$\Phi_{\nu} \quad$ The neutrino flux while in neutrino mode

$\Phi_{\text {Off }} \quad$ The neutrino flux while in off-target mode

$G_{N} \quad$ Newton's gravitional constant

$H \quad$ Hubble's parameter (time dependent)

$h \quad$ The reduced Hubble's constant set equal to $H_{0} /\left(100 \mathrm{~km} \mathrm{~s}^{-1} \mathrm{Mpc}^{-1}\right)$

$H_{0} \quad$ Hubble's constant

$n \quad$ Index of refraction

$\mathcal{L} \quad$ Lagrangian

$\Lambda \quad$ The cosmological constant used in general relativity

L Likelihood function

$-2 \ln L \quad$ Negative of the log-likelihood times two

$\Delta(-2 \ln L) \quad$ The $\log$ ratio of two likelihood values

$\Delta(-2 \ln L)_{\text {Null }}$ The difference between the null likelihood value and the best fit likeli-

hood value

$\Delta(-2 \ln L)_{\text {Sig }} \quad$ The difference between the signal likelihood value and the best fit like-

lihood value

$m_{\chi} \quad$ Mass of the dark matter particle

$m_{V} \quad$ Mass of the dark matter mediator

$m_{\mu} \quad$ Mass of the muon

$m_{n} \quad$ Mass of the neutron

$m_{n}^{\prime} \quad$ Adjusted mass of the neutron equal to $m_{n}-E_{b}$ 


$\begin{array}{ll}m_{P} & \text { Planck mass defined as } 1 / \sqrt{8 \pi G_{N}} \\ m_{p} & \text { Mass of the proton } \\ m_{Z} & \text { Mass of the Z-boson } \\ \text { NCE }_{\text {Off }} & \text { The NCE sample from the off-target data set } \\ \text { NCE }_{\nu} & \text { The NCE sample from the } \nu \text {-Mode data set } \\ \nu-\text { Mode } & \text { Data taken while the BNB horn was in neutrino mode } \\ \Omega_{b} & \text { The cosmological baryonic density } \\ \Omega_{b} h^{2} & \text { The cosmological reduced baryonic density } \\ \Omega_{c} & \text { The cosmological dark matter density } \\ \Omega_{c} h^{2} & \text { The cosmological reduced dark matter density } \\ \Omega_{\Lambda} & \text { The cosmological dark energy density } \\ Q_{Q E}^{2} & \text { The reconstructed four momentum transer based off the quasi-elastic } \\ & \text { assumption } \\ T & \text { Temperature } \\ V & \text { Dark matter mediator where the mass is given by } m_{V}\end{array}$




\section{LIST OF APPENDICES}

A Energy-Dependent $L_{t}^{p / e}$ Cut Study 131

B Effect of Correlations on Fits 133

B.1 Fit Calculations . . . . . . . . . . . . . . . . . . 133

B.2 Conditions on $\theta_{\min } \ldots \ldots \ldots \ldots \ldots \ldots \ldots \ldots \ldots$

C Singular Value Decomposition $\quad 135$ 


\section{LIST OF FIGURES}

1.1 Rotation curve of NGC 3198. From Ref. [3] . . . . . . . . . . . . . 2

1.2 The cosmic microwave background (CMB) temperature power spectrum from 2015 Planck results $[5] \ldots \ldots \ldots$. . . . . . . . . . . . 4

2.1 Schematic view of the role of $\epsilon$ and $\alpha^{\prime}$ in light dark matter (LDM). . . . 10

2.2 Feynman diagram showing two complex scalars annihilating into standard model $(\mathrm{SM})$ particles. . . . . . . . . . . . . . . . . . . 10

2.3 One-loop correction to the $\gamma$-fermion vertex in QED. . . . . . . . . . . 11

2.4 Production of dark matter (DM) in a proton fixed-target/beam dump beam-

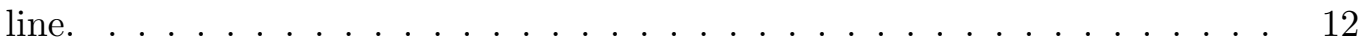

2.5 Interaction channels in LDM considered in this analysis. . . . . . . . . . 13

2.6 Current best limits in direct DM weakly interacting massive particle (WIMP) searches. . . . . . . . . . . . . . . . . . . . . 14

2.7 Visible decay limits. Dashed lines are predicted sensitivities for Jefferson National Laboratory (JLab) and Large Hadron Collider (LHC) experiments. The Seaquest regions are also predicted. All other curves are current limits. From Ref. [38]. . . . . . . . . . . . . . . . . . . . . 16

3.1 Schematic of how the neutrinos are produced at the Booster Neutrino Beamline $(\mathrm{BNB})$ with respect to the MiniBooNE detector. . . . . . . . . . . . 20

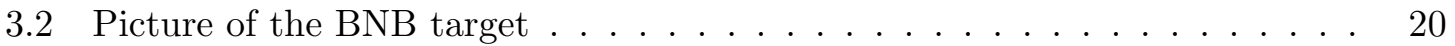

3.3 Results of a BNB target scan. . . . . . . . . . . . . . . . . . . . . . 21

3.4 The $\nu$-Mode flux as a function of true $\nu$ energy predicted by the BooNEG4BEAM simulation package. . . . . . . . . . . . . . . . . . . 24 
3.5 A schematic of the MiniBooNE detector showing (left) the cut-away drawing and (right) the location of the photomultiplier tubes as well as the separation between the signal and veto regions. Copied from Ref. [68] . . . . . . .

3.6 Checking the scaling of STROBE triggers to BEAM triggers. The blue line (top plot top line) is data from STROBE while the orange line (top plot bottom line) is data from BEAM. The axis is time in the data acquisition (DAQ) window. The green vertical lines and arrows shows the beam time cut discussed in Sec. 4.1.1. The bottom plot is the ratio of BEAM to STROBE it is in good agreement with what one would expect after adjusting the number of BEAM triggers to account for empty triggers. . . . . . . . . . . .

3.7 The geometry domain of the MiniBooNE GEANT3 simulation. The dirt region and overburden are indicated by the thick-lined cylinder and the conical frustum, the thin-lined cylinders indicate the electronics room and vault, the sphere and the top-hat determine the detector tank and the tophat. From

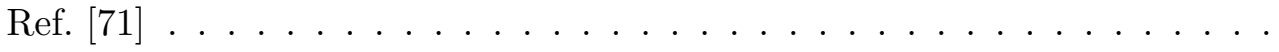

3.8 Schematic of the reconstructed geometry defining the values that go into $\mathbf{X}$. 34

4.1 Cartoon of what a typical (a) neutral-current elastic (NCE) and (b) chargedcurrent quasielastic (CCQE) events contains in the MiniBooNE detector. .

4.2 Diagram showing how previous trigger Clusters are made. . . . . . . . .

4.3 Example time difference between current event and previous triggers distributions for beam and beam-unrelated data where the previous trigger has the DET5 bit set. (a) Uses the normal time likelihood cut while (b) takes the

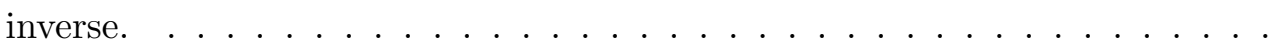


4.4 Percent change of $\mathcal{F}$ after applying a pre-trigger cut as a function of DET, VETO, and $\Delta T$. A DET0 or VETO0 means there was no constraint on that type of trigger, i.e. DET0 and VETO0 means any trigger not caused by an external

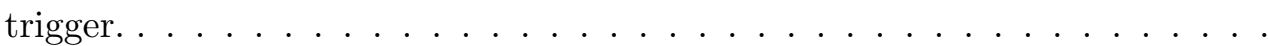

4.5 Schematic of a tube shape material in the simulations. The dark outer cylinder is the material of radius $r_{o}$ and the inner white cylinder is hollow with radius $r_{i}$. The half length of the tube (half $\left.-z\right)$ is also shown. . . . . .

4.6 Simulated beamline geometry. (b), (c), and (d) are zoomed in parts shown in (a). The zoomed in parts are not zoomed to the same scale. . . . . . .

4.7 $3-\sigma$ Projections of the beam with various starting parameters given at various

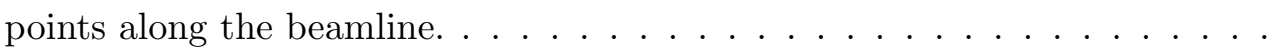

4.8 Comparing $\mathrm{CCQE}_{\mathrm{Off}} E_{\nu}^{\mathrm{QE}}$ data to three different Monte Carlo Predictions. Dotted red is the output of the nominal off-target beam profile, dashed red is the dotted red scaled by 1.62 , solid blue is the final central-value flux determined by averaging excursions. . . . . . . . . . . . .

4.9 (top) The $E_{\nu}$ distribution of the flux in off-target mode. (bottom) The ratio of off-target to $\nu$-Mode flux. Both show each neutrino species. . . . . . . . 53

4.10 (a) The total off-target central-value flux distribution with stat and beam profile systematic errors. (b) The total beam profile systematic correlation

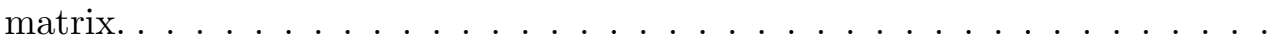

4.11 Predicted $\nu_{e}$ (top right), $\bar{\nu}_{e}$ (top left), $\nu_{\mu}$ (bottom left), and $\bar{\nu}_{\mu}$ (bottom right) flux at the MiniBooNE detector by secondary particle in beam offtarget mode. The black line is the total predicted flux. The rest of the lines (except the dashed black line) are from primary parent meson production. The dashed black line includes all other contributions. . . . . . . . . . . 
$4.12 E_{\nu}^{\text {true }}$ distributions for true (a) NCE and (n) CCQE events. The bold lines are after the corresponding selection cuts are made and the thinner lines are before detector effects and selection cuts. For each set $\nu$-Mode and off-target

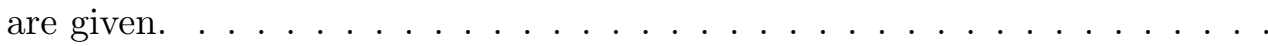

4.13 The average of the $\pi^{ \pm}$produced in the BooNEG4BEAm simulation, used as the $\pi^{0}$ distribution in simulating DM, for off-target and $\nu$-Mode. . . . .

$4.14 \Delta \chi_{\min }^{2}$ distributions of the NBP flux compared to data (solid black curve) and $\overline{C V}$ flux compared to data (long dashed black curve) when varying $b$. The scale applied to $\mathbf{E}^{\prime}$ is shown for NBP (solid gray curve) and for $\overline{C V}$ (long dashed gray curve). The black square marker shows where on the NBP flux $\Delta \chi_{\min }$ curve $b=0$ and the black circle marker shows where, on the same curve, $b=b_{\min _{\overline{C V}}}$. Horizontal gray dashed lines make it easier to see the flux-factor scale for NBP and $\overline{C V}$ when $b=0$. The scale for the flux-factors is given on the right while the scale for $\Delta \chi_{\min }^{2}$ is given on the left. . . . .

4.15 Example of the individual correlation matrices from the systematic uncertainty studies. Cross-section and Optical Model systematics are shown. All four distributions are plotted as functions of $Q_{Q E}^{2} \ldots \ldots \ldots$

4.16 The energy dependent systematic uncertainty correlation matrix between the four datasets. . . . . . . . . . . . . . .

4.17 The NCE/CCQE $\nu$-Mode/off-target distributions. Statistical uncertainty are shown in black and systematic uncertainty are shown in gray. . . . . . .

5.1 The data and total background predicted distributions for $\mathrm{CCQE}_{\nu}, \mathrm{NCE}_{\nu}$, $\mathrm{CCQE}_{\mathrm{Off}}, \mathrm{NCE}_{\mathrm{Off}}$ as a (a) chain and (b) ratio in $Q_{Q E}^{2} \ldots \ldots$

5.2 Schematic of how to generate fake data $\ldots \ldots \ldots$ 
5.3 The ratio of $\mathrm{NCE}_{\nu}$, after beam-unrelated background (BUB) and $\nu_{\text {dirt }}$ subtraction, data to $\nu_{\text {det }}$ as a function of $Q_{Q E}^{2}$ (black histogram) with fit results of the fit to Eq. (5.1) (red line) . . . . . . . . . . . . . 76

5.4 The results of the background-only fit. . . . . . . . . . .

5.5 The cumulative probability distribution for signal best fit and null fit to null fake data sets. This shows that the probability that the prediction compared to data yields a test statistic this small (or smaller) is $3 \% \ldots \ldots \ldots$

5.6 The cumulative distribution function distribution for the constrained fit test statistic when fitting null fake data with the null hypothesis, and where the background-only (null) fit to data falls in the cumulative distribution function distribution. The probability data is consistent with simulation (MC) predictions using the constrained fit is $8 \% \ldots \ldots \ldots$

5.7 The correlation matrix for (a) fit parameters in background-only fit and (b) number of events after propagating the best fit parameters. . . . . . . .

5.8 (a) $T_{p}^{\text {true }}$ coming from NUANCE, shown with the extrapolated distribution used to simulate every scattering is off a free nucleon. (b) Total efficiency of NCE events in the MiniBooNE detector. The total efficiency is the product of detector threshold, NCE selection criteria, and nuclear model so that results of model independent fit (MIF) and confidence limit are corrected to free nucleon scattering. . . . . . . . . . . . . .

5.9 (a) The predicted $T_{N}^{\text {true }}$ coming from the MiniBooNE NUANCE simulation extrapolating to $0 \mathrm{GeV}$ as stated in Fig. 5.8a. (b) The same distribution but rebinned into the bins used in the excess search. . . . . . . . . .

5.10 (a) The smearing matrix (to go from $Q_{Q E}^{2} \leftrightarrow T_{N}^{\text {true }}$ ) for NCE off-target $Q_{Q E}^{2}$ binning and the fine $T_{N}^{\text {true }}$ binning. (b) Same as (a) but with the coarse $T_{N}^{\text {true }} .89$ 
5.11 (a) Showing the $\varepsilon$ distribution given by Eq. (5.10), as well as (b) the $S^{\varepsilon}$ matrix shown with NCE off-target $Q_{Q E}^{2}$ binning. . . . . . . . . . . . 90

5.12 The results of the MIF as a function of $T_{N}^{\text {true }} \ldots \ldots \ldots \ldots$. . . . . 91

5.13 The true radius position for (a) $\mathrm{NUANCE}=1$ events passing CCQE cuts and (b) NUANCE $=2$ events passing NCE cuts. . . . . . . . . .

5.14 The excess number of events/(1e20 protons on target (POT)) as a function of reconstructed $Q_{Q E}^{2}$. The black points with error bars shows the data in Fig. 4.17a subtracted by the total prediction. The error bars on the black points is total uncertainty. The dotted line gives the results when adjusting the prediction by the initial guess nuisance parameters. The long dashed line gives the results of the MIF. The light dark matter (LDM) region of interest (ROI) is to the left of the vertical gray line. . . . . . . . . . . .

6.1 Schematic of vector portal LDM being produced in the off-target running configuration through $\pi^{0}$ decay. . . . . . . . . . . . . .

6.2 Ratio of number of protons to number of neutrons produced in DM-nucleon scattering with a low nuclear recoil cut off at $20 \mathrm{MeV} . \epsilon=10^{-3}$ and $\alpha^{\prime}=0.1 .97$

6.3 Predicted number of nucleons from DM scattering in MiniBooNE, assuming $2 \times 10^{20} \mathrm{POT}$, as a function of $m_{V}$ with $m_{\chi}=10 \mathrm{MeV}, \epsilon=10^{-3}$, and $\alpha^{\prime}=$ 0.1. Copied from $[28] . \ldots \ldots \ldots \ldots$. . . . . . . . . . . . . . . . 98

6.4 The predicted number of events/(1e20 POT) and mean true nucleon recoil energy coming out of BDNMC. Both plots use an efficiency equal to 1 as well as $\epsilon=10^{-3}$ and $\alpha^{\prime}=0.1$. The lighter $m_{V}, m_{\chi}$ combination the softer the recoil nucleon energy spectrum. . . . . . . . . . . . . .

6.5 (a) Produced $T_{N}^{\text {true }}$ distribution from BDNMC for different combinations of $m_{V}, m_{\chi}$. (b) Reweighted DM prediction as a function of $Q_{Q E}^{2}$ for the same combinations of $m_{V}, m_{\chi}$ used in (a). Both plots have $\epsilon=10^{-3}$ and $\alpha^{\prime}=0.1 .100$ 
6.6 The percent difference (a) corresponding $-\left(\epsilon^{4} \alpha^{\prime}\right)$ (b) as a function of $m_{V}$, $m_{\chi}$ are the results of the best fit to data. . . . . . . . . . 103

6.7 An illustration of the confidence limit (CL) procedure for a given $m_{V}, m_{\chi}$ combination. The data distribution is given by black points, the $90 \%$ values from frequentist fake data is the black line, a linear fit to the fake data is the red dashed line, the traditional $\chi^{2}$ value used is given by the solid orange line, and the predicted sensitivity using SensMethod1 is shown as a vertical blue dotted line. . . . . . . . . . . . . . . . . . . . . . . . . 104

6.8 (a) The null fake data $\Delta(-2 \ln L)_{\text {Null }}$ distribution compared to $\chi^{2}$ distributions with different number degrees of freedom, and (b) the probability distribution function of $\Delta(-2 \ln L)_{\mathrm{Null}}$ for different $\epsilon^{4} \alpha^{\prime}$ fake data. The vertical red line in (b) is the same as the vertical red line in (a) . . . . . . 106

6.9 The corresponding cumulative distribution function (CDF) where the vertical black line is the calculated mean and the green (yellow) regions are the $\pm 1 \sigma( \pm 2 \sigma)$ calculated limits. . . . . . . . . . . . . . . 108

6.10 Comparing SensMethod1 and SensMethod2 for (a) $Y$ vs. $m_{\chi}$ with $m_{V}=$ $3 m_{\chi}$ and $\alpha^{\prime}=0.5$, and (b) $\epsilon$ vs. $m_{V}$ with $m_{\chi}=10 \mathrm{MeV}$ and $\alpha^{\prime}=0.1 . . .109$

6.11 Comparing the two CL and sensitivity methods for (a) $Y$ vs. $m_{\chi}$ with $m_{V}=3 m_{\chi}$ and $\alpha^{\prime}=0.5$, and (b) $\epsilon$ vs. $m_{V}$ with $m_{\chi}=10 \mathrm{MeV}$ and $\alpha^{\prime}=0.1 .110$

6.12 The $90 \%$ CL on $\epsilon^{4} \alpha^{\prime}$ for each set of $m_{V}, m_{\chi}$ from using the (a) traditional and (b) frequentist approaches. The sensitivity is shown in (c) for SensMethod1 and $(\mathrm{d})$ for SensMethod2. . . . . . . . . . . . . . .

6.13 The CDF function for signal fake data where the signal parameters are set to the overall best fit results and the result from the signal best fit constrained fit. The probability that data is consistent with background predictions plus best fit dark matter using the constrained fit is $19 \% \ldots \ldots \ldots \ldots$ 
7.1 (a) The $90 \%$ CL on $\epsilon^{4} \alpha^{\prime}$ for each set of $m_{V}, m_{\chi}$ from using the frequentist approach discussed in Sec. 6.2. (b) The $90 \%$ sensitivity on $\epsilon^{4} \alpha^{\prime}$ for the same combinations of $m_{V}, m_{\chi}$ as in (a). . . . . . . . . . . . . 114

7.2 Slices of Fig. 6.12 in $Y$ vs. $m_{\chi}$ with a fixed ratio of $m_{\chi} / m_{V}$ and fixed $\alpha^{\prime}$. The blue shaded region is where this model reduces the muon g-2 discrepancy between theory and experiment to $\pm 3 \sigma$ [29-31]. E137 was an electron beam dump at SLAC originally searching for axions $[53,55]$. LSND was a stop pion neutrino detector at LANCE $[25,46,102]$. Direct detection includes limits from the most sensitive low mass results, currently from CDMSlight [103] and CRESST-II [34] for nucleon recoils and Xenon10 [104] for electron recoil. $K^{+} \rightarrow \pi^{+}+$invisible was a rare kion decay search done at BNL (E949) [32, $53,105] . \quad J / \Psi \rightarrow$ invisible was done by BES searching for missing energy in the $J / \Psi$ decay [106]. BaBar was a reinterpretation of $\Upsilon(3 S) \rightarrow \gamma+$ invisible [107, 108]. NA64 searched for missing energy [109]. Dot-dashed lines rely on DM coupling to leptons. . . . . . . . . . . . . . . 115

7.3 Slices of Fig. 6.12 in $\epsilon$ vs. $m_{V}$ with a fixed $m_{\chi}$ and $\alpha^{\prime}$. The blue shaded region is where this model reduces the muon g-2 discrepancy between theory and experiment to $\pm 3 \sigma$ [29-31]. E137 was an electron beam dump at SLAC originally searching for axions $[53,55]$. LSND was a stop pion neutrino detector at LANCE $[25,46,102] . K^{+} \rightarrow \pi^{+}+$invisible was a rare kion decay search done at BNL (E949) [32, 53, 105]. BaBar was a reinterpretation of $\Upsilon(3 S) \rightarrow \gamma+$ invisible [107, 108]. NA64 searched for missing energy [109]. Direct detection electron is from Xenon10 [104]. Dot-dashed lines rely on DM coupling to leptons. . . . . . . . . . . . . . . . . . . . 116

7.4 Leptophobic 90\% CL using the MIF results. Explanation of the previous limits are given in Ref. [110]. . . . . . . . . . . . . . . . . . 117 
A.1 (a) The $L_{p / e}^{t}$ distribution broken down by channel; beam unrelated background, neutrino NCE interactions, neutrino NCE-like interactions, and neutrino interactions in the dirt. The vertical line and arrow shows where the cut is placed and which events are kept after the cut. All other cuts are applied. (b) $L_{t}^{p / e}$ vs $T_{N}^{r e c o}$ where the columns are differences to the minimum $\mathcal{F}$ in that column. The white line shows the original cut, the tan vertical lines show the $T_{N}^{\text {reco }}$ cut, the dashed yellow (magenta) line shows the results of fitting the minimum in each bin with a 4th- (6th-) order polynomial. . . 132 


\section{LIST OF TABLES}

2.1 Previous/Current/Future experiments on proton beamlines where limits on LDM have/will be set. For each experiment, the amount of beam, target material, beam energy, and the experiment run period are listed. . . . . .

2.2 Previous/Current/Future experiments on proton beamlines where limits on LDM have/will be set. For each experiment, the amount of beam, target material, beam energy, and the experiment run period are listed. . . . . . .

3.1 The $\pi^{+}$and $\pi^{-}$Sanford-Wang distribution (SW) parameters $c_{1-9}$ used in the BooNEG4Beam simulation package. Copied from Ref. [62] . . . . . .

3.2 Interaction and Collision lengths for the various materials in the BNB. All values are given in meters $[67] \ldots \ldots \ldots \ldots \ldots$

3.3 The three external and seven comparator trigger settings. Copied from Ref. $[68] \ldots \ldots \ldots \ldots \ldots \ldots \ldots \ldots \ldots \ldots$

3.4 The NUANCE parameters used to generate the $\nu$-Mode detector simulation $(\operatorname{det} \mathrm{MC})$ events. $\mathrm{QE}=$ quasi-elastic, $\mathrm{CC}=$ charge-current, $\mathrm{NC}=$ neutralcurrent. Copied from Ref. $[76] \ldots \ldots \ldots \ldots \ldots$

4.1 List of cuts for the $\mathrm{NCE}$ and CCQE event selections. . . . . . . . . . . .

4.2 List of geometries upstream or around the target needed for off-target simulations. The location is with respect to the center of the target. When there is multiple information given for a specific geometry that is either because there are multiple pieces not connected, e.g. MWTGT foils, or the geometry spans over several mother volumes, e.g. Vacuum. BCA stands for bellows contact assembly. . . . . . . . . . . . . . . . . 
4.3 The nominal beam off-target staring parameters, where the uncertainties given are RMS errors for the given parameter. . . . . . . . . . . . . .

4.4 Beam off-target profile systematic percent error independent of energy for the various $\nu$ types, including correlations. $\Phi\left(E_{\nu}\right)$ integrated over $0.2<$

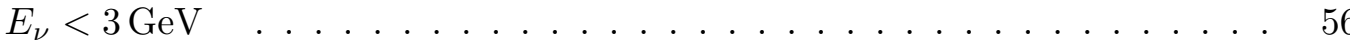

4.5 Predicted $\nu_{\mu} / \bar{\nu}_{\mu}$ (top) and $\nu_{e} / \bar{\nu}_{e}$ (bottom) fluxes at MiniBooNE detector while in beam off-target beam configuration. The contribution flux of the decay chains are provided for the primary parent meson production. . . . .

4.6 Breakdown of predicted $\nu_{\text {det }}$ by true neutrino interaction given by NUANCE. The first column gives the percentage of total events of each type, as determined at the primary vertex (not counting any final state interactions). The second and third columns gives the percentage of each event type for NCE and CCQE cuts. Other includes multiple $\pi$ production, deep inelastic scattering, elastic scattering off an electron, and delta radiative decay. . . . 58

4.7 The breakdown of the total number of $\pi^{ \pm}$per POT and by material in the beamline. A pion was counted if it had a total kinetic energy greater than $1 \mathrm{MeV}$, was traveling in the forward direction, and had a transverse momentum less than $1 \mathrm{GeV}$. Off-target, in this table, refers to the nominal beam position measured by the multiwires, not the average of the four excursions that is used as the neutrino flux CV. . . . . . . . . . . . .

4.8 Percent systematic uncertainty on total number of events for each channel

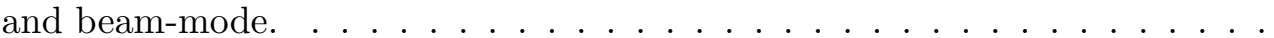

4.9 The integral number of events for each distribution. The experimental data, total background, breakdown of background, and fit results are given. The fit results are discussed in Sec. $5.2 \ldots \ldots \ldots \ldots \ldots$

5.1 The symbol and name of the nuisance parameters used for the analysis. . . 
5.2 The numerical values of the nuisance parameters from the initial guess and the background-only fits shown in Fig. 5.4. . . . . . . . . . . . . 78

5.3 The systematic and statistical uncertainty for each data set along with the propagated uncertainty looping only over the given data sets block. . . . . . 


\section{CHAPTER 1}

\section{INTRODUCTION}

Dark matter (DM) was initially proposed by Fritz Zwicky in 1933 [1]. Zwicky combined Hubble's results (using redshift to calculate distances) and van Maaen's results (calculating the angular movements of a cluster) to calculate the mass-to-light ratio of the Coma cluster. For both Hubble and van Maanen to be correct, Zwicky concluded the total matter density needed to be about 400 times the amount given by luminous matter alone; modern observations observe the total mass density to be 200 to 300 times the luminous density. Zwicky explained the difference by introducing a dark particle. Sinclair Smith did the same calculation for the Virgo cluster in 1936 [2], but it took another 35 years for the physics community to start accepting DM to be universal.

Today, evidence for DM comes from a wide range of cosmological scales including galaxies, clusters, cosmic microwave background (CMB), and gravitational lensing. For example, the Doppler shift of the $21 \mathrm{~cm}$ line from hydrogen is used to calculate rotation curves for single galaxies. Fig. 1.1 shows the rotation curve for NGC 3198. The main characteristic of the rotation curves is the long flat component that extends for most of the distribution. The best fit, shown in Fig. 1.1, is when the rotational curve is composed of a convolution of disk and halo like mass densities. The data cannot be explained fully by a mass density consistent of a disk, i.e. ordinary matter cannot fully explain the curve. The halo line is an assumption for the mass density of DM in the galaxy. At $30 \mathrm{kpc}$ the DM density is 


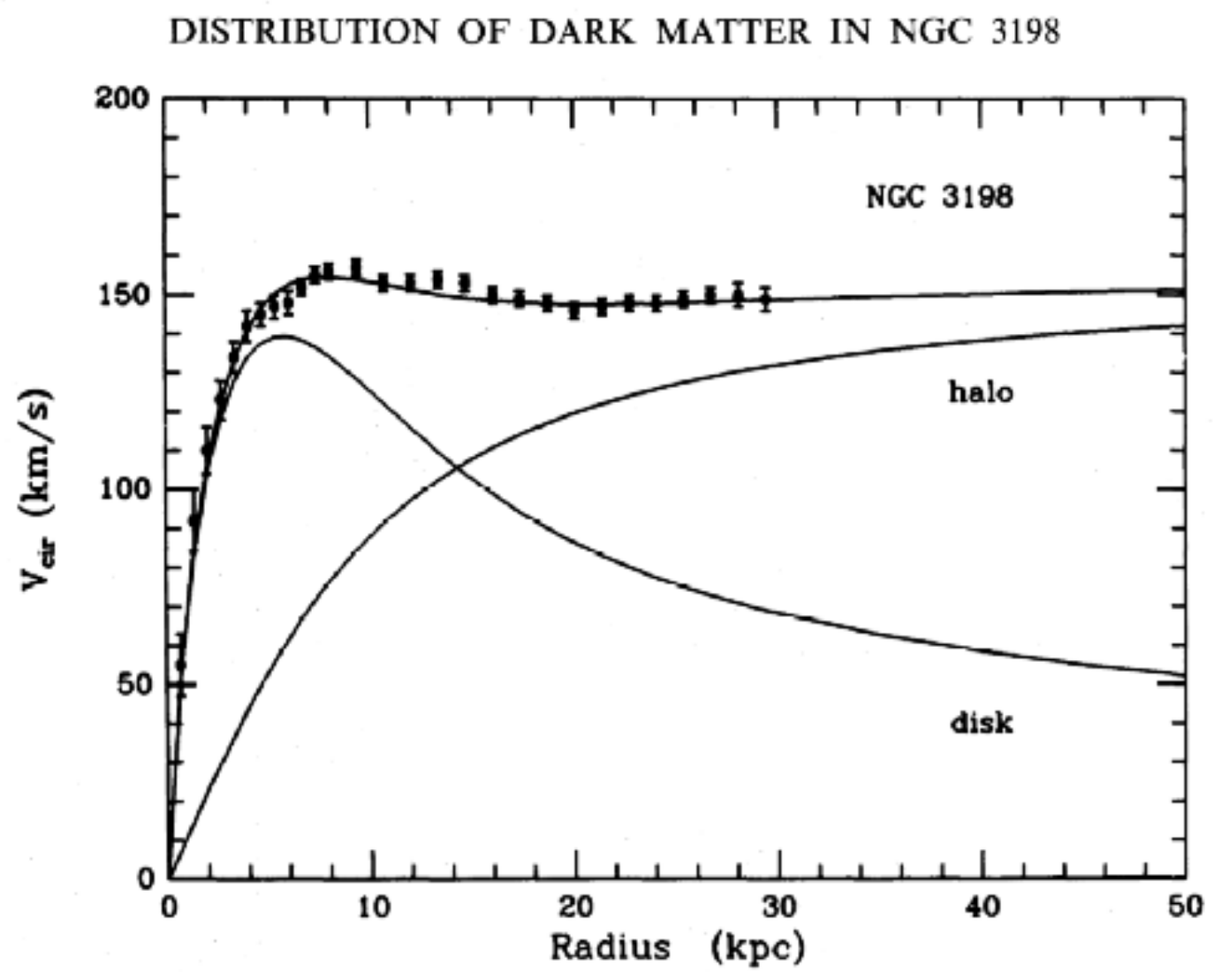

Figure 1.1: Rotation curve of NGC 3198. From Ref. [3] 
measured to be four times the amount of ordinary matter. Coma and Virgo are examples of galaxy clusters where evidence of DM is seen.

The CMB is known to be isotropic to $10^{-5}$, once the galactic plane and dipole contributions are subtracted [4]. The observed temperature anisotropies are expanded by spherical harmonics $Y_{l m}(\theta, \phi)$ given by

$$
\frac{\delta T}{T}(0, \phi)=\sum_{l=2}^{+\infty} \sum_{m=-l}^{+l} a_{l m} Y_{l m}(0, \phi),
$$

where the variance on $a_{l m}$ is given by

$$
C_{l} \equiv\left\langle\left|a_{l m}\right|^{2}\right\rangle \equiv \frac{1}{2 l+1} \sum_{m=-l}^{+l}\left|a_{l m}\right|^{2} .
$$

The multipole $l$ is related to the distance between nodes by

$$
l=k d_{A}^{c},
$$

where $k$ is the wave number, $d_{A}^{c}$ is the comoving angular diameter distance defined by

$$
d_{A}^{c} \equiv \frac{\lambda^{c}}{\theta}=\frac{(1+z) \lambda_{\text {phys }} l}{2 \pi} .
$$

The power spectrum of the CMB, Fig. 1.2 , shows $D_{l} \equiv l(l+1) C_{l} / 2 \pi$ vs $l[4]$. The peaks in the power spectrum measure different properties of the universe, which can be determined by knowing how various properties effect different length scales. The relative size between the first two peaks is dependent on how much of the universe is baryons. The first peak also provides information about the shape of the universe. The remaining peaks, if measured well, characterize how much non-relativistic matter there is in the universe. Combining the temperature power spectrum with the polarization and temperaturepolarization cross-correlation power spectra the Planck Collaboration (Planck) fit the data to the Lambda-Cold Dark Matter $(\Lambda \mathrm{CDM})$ cosmological model to determine various cosmological parameters [5].

$\Lambda \mathrm{CDM}$ is considered the standard model of Big Bang cosmology that incorporates the cosmological constant $\Lambda$ representing dark energy and cold dark matter into general 


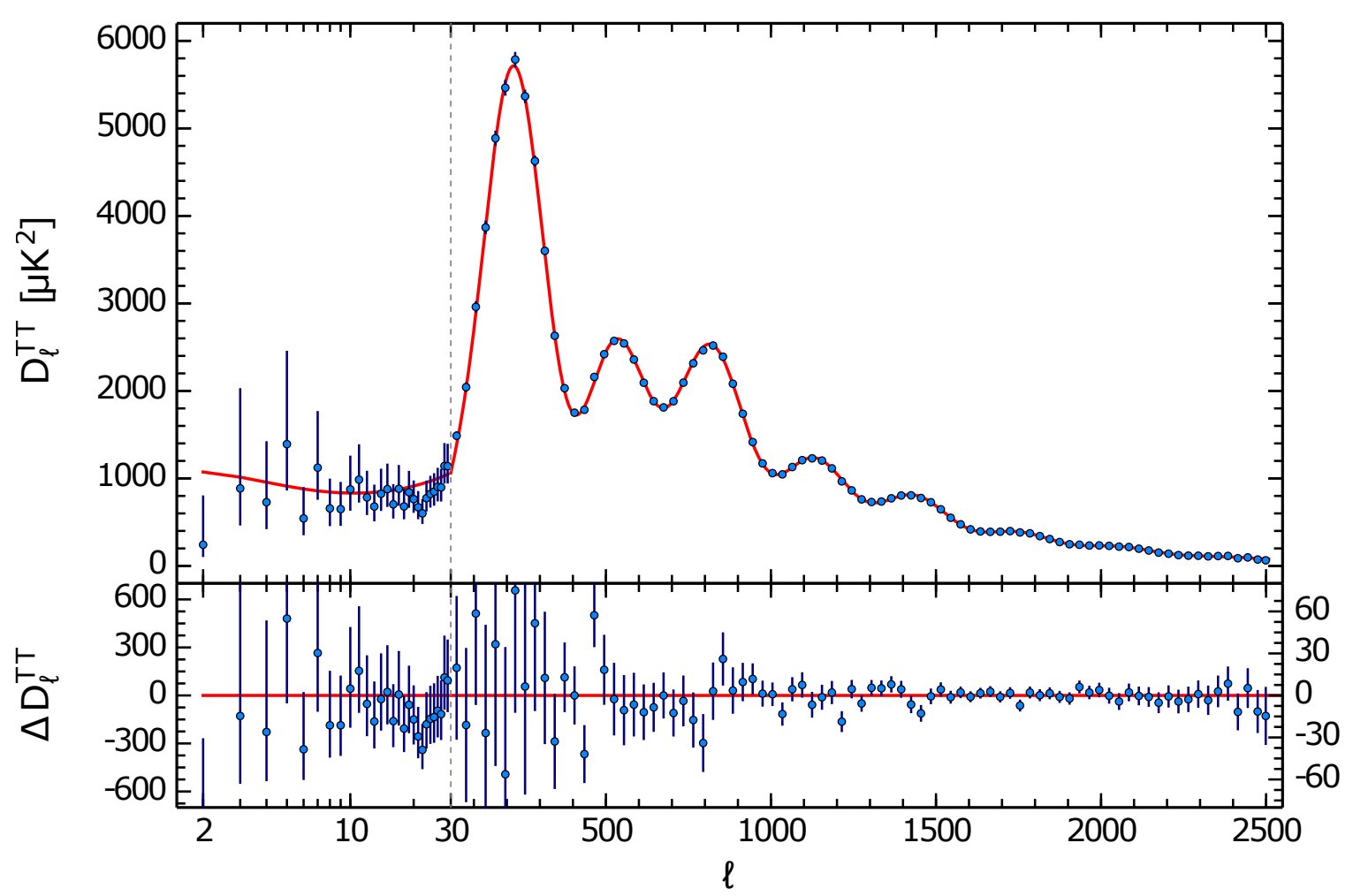

Figure 1.2: The CMB temperature power spectrum from 2015 Planck results [5].

relativity. For a specifically flat $\Lambda \mathrm{CDM}$ model, the model consists of six fit parameters; two of the fit parameters are the physical baryon density $\Omega_{b} h^{2}$ and the physical DM density $\Omega_{c} h^{2}[5] . \Omega_{x}$ is defined as the ratio of the density of $x$ and the critical density of the universe $\rho_{\text {crit }}$, and the critical density of the universe is the needed energy/matter density to produce a flat universe. $\rho_{\text {crit }}$ is approximately equal to $10^{-6} \mathrm{GeV} \mathrm{cm}^{-3}[4,6]$. Hubble's constant $H_{0}$ and the dark energy baryon density parameter $\Omega_{\Lambda}$, along with many other parameters, are derived from the fit parameters, where the reduced Hubble's constant is defined as $h \equiv H_{0} /\left(100 \mathrm{~km} \mathrm{~s}^{-1} \mathrm{Mpc}^{-1}\right)$. Planck measured $\Omega_{b} h^{2}=0.02230 \pm 0.00014$, $\Omega_{c} h^{2}=0.1188 \pm 0.0010, H_{0}=(67.70 \pm 0.46) \mathrm{km} \mathrm{s}^{-1} \mathrm{Mpc}^{-1}$, and $\Omega_{\Lambda}=0.6911 \pm 0.0062[7]$. Using the measured $H_{0}$ to calculate $\Omega_{b}, \Omega_{c}$, and $\Omega_{\Lambda}$, gives the current measured composition of the universe to be $69.1 \%$ dark energy, $25.9 \%$ DM, and $4.9 \%$ baryonic matter [7].

Dark matter evidence also comes from gravitational lensing. The Bullet cluster is an 
example of leftover mass seen from two galaxy clusters colliding. After taking into account gravitational lensing, the mass distribution is measured to be concentrated further away from the "collision" point than what X-ray data suggests [8].

With all the evidence in cosmology for DM, DM interactions with the particles in the standard model of particle physics remains a mystery. The subject of this work is to search for interactions of DM, produced in a proton beam dump, with standard model particles in the MiniBooNE detector. Chapter 2 will highlight several proposed theories and their limitations, and describe in more detail the theory tested by this analysis. Chapter 3 gives the experimental setup that was used in this analysis. Chapters 4 to 6 describes the analysis cuts, fit procedure, and method for determining confidence limits. Chapter 7 compares this result to previous experiments. There is also a set of appendices to provide more details of the analysis. 


\section{CHAPTER 2}

\section{DARK MATTER THEORY}

There is a zoo of theories predicting how dark matter (DM) interacts within the standard model (SM). This chapter starts with an order of magnitude calculation to determine a likely cross secion/mass scale for a DM search, and an overview of some of the proposed theories. A detailed look into the theory tested in this analysis is then presented followed by a discussion of previous experimental data that has been used to set limits on the benchmark model.

\subsection{ORDER OF MAGNITUDE CALCULATION}

The following calculation follows from Ref. [6]. Independent of the DM model, the number density for DM should follow

$$
\begin{array}{r}
n_{r e l} \sim T^{3} \text { for } m_{\chi} \ll T, \\
n_{n o n-r e l} \sim\left(m_{\chi} T\right)^{3 / 2} \exp \left(-\frac{m_{\chi}}{T}\right) \text { for } m_{\chi} \gg T,
\end{array}
$$

where $m_{\chi}$ is the mass of the DM particle $\chi$, and $T$ is the temperature of the thermal bath in which the DM sits [6]. Setting the following assumptions:

1. the decay rate of producing standard model particles from DM annihilation/decay $\Gamma$ is equal to the DM number density times the DM interaction cross section, $\Gamma=n \sigma$,

2. $\Gamma$ is equal to the Hubble parameter $H$, 
3. DM is cold, $m_{\chi} \gg T$,

allows the following condition on the number of DM particles at freeze-out

$$
n_{f . o .} \sim \frac{T_{f . o .}^{2}}{m_{P} \cdot \sigma},
$$

where $m_{P}=1 / \sqrt{8 \pi G_{N}}$, and $G_{N}$ is Newton's gravitational constant [6]. Freeze-out is when the probability of two particles annihilating becomes insignificant due to the temperature of the universe cooling causing $n$ to become small. Eq. (2.1) can be rewritten as

$$
\frac{m_{\chi}^{3}}{x^{3 / 2}} e^{-x}=\frac{m_{\chi}^{2}}{x^{2} \cdot m_{P} \cdot \sigma},
$$

where $x \equiv m_{\chi} / T$ and assuming DM is nonrelativistic. Solving for $x$ produces

$$
\sqrt{x} e^{-x}=\frac{1}{m_{\chi} \cdot m_{P} \cdot \sigma}
$$

which is dependent on the values of $m_{\chi}$ and $\sigma$. If an "electro-weak interacting" DM particle is assumed, i.e. $\sigma \sim G_{F}^{2} m_{\chi}^{2}$ with $m_{\chi}$ between 4.6 and $10^{4} \mathrm{GeV}, x$ has a value between 20 and $50[6]$.

By definition the DM relic density is equal to

$$
\Omega_{c}=\frac{\rho_{\chi}}{\rho_{\text {crit }}}
$$

Knowing that $\rho_{\chi}=m_{\chi} n_{\chi}\left(T=T_{0}\right), \Omega_{c}$ can be rewritten as

$$
\Omega_{c}=\frac{m_{\chi} n_{\chi}\left(T=T_{0}\right)}{\rho_{c r i t}}
$$

where $T_{0}=2.75 \mathrm{~K} \sim 10^{-4} \mathrm{eV}$. Assuming an iso-entropic universe, where acceleration of the universe times the temperature of the universe is constant, the ratio of $n / T^{3}$ at freeze-out is the same as $T_{0}[6]$. Applying the iso-entropic universe approximation along with known values for $\rho_{\text {crit }}, m_{P}$, and $T_{0}$, produces

$$
\frac{\Omega_{c}}{0.2} \simeq \frac{x_{f . o .}}{20}\left(\frac{10^{-8} \mathrm{GeV}^{-2}}{\sigma}\right)
$$


Substituting $\sigma v$ for $\sigma$ in Eq. (2.2) produces

$$
\langle\sigma v\rangle \sim 3 \times 10^{-26} \mathrm{~cm}^{3} \mathrm{~s}^{-1}
$$

where $\Omega_{c} \sim 0.2$, and $v \sim c / 3$ for $x \sim 20$.

Eq. (2.2) tells us that $0.1 \mathrm{eV} \ll m_{\chi}<120 \mathrm{TeV}$ if $\sigma \sim 10^{-8} \mathrm{GeV}^{-2}$; this leads to the weakly interacting massive particle (WIMP) "miracle" because the electro-weak cross section is on the order of $10^{-8} \mathrm{GeV}^{-2}$. Many DM candidates have been proposed and Eq. (2.3) is used as a guide to see if the candidate is valid or not.

\subsection{CANDIDATE DARK MATTER MODELS}

The discussion of this section follows that of Ref. [4]. References listed are taken from Ref. [4]. Supersymmetry particles are a popular choice for DM. The supersymmetric candidates include neutralinos, sneutrinos, gravitinos, and axinos. Neutralinos have a large possible DM parameter space because of the large number of free parameters in the minimal supersymmetric standard model. Neutralinos are made up of four Majorana fermionic mass eigenstates composed by the superpartners of the photon, Z-boson, and neutral Higgs bosons [4]. Sneutrinos are ruled out due to a high cross section with the nucleon [9]. Gravitinos require a low temperature in the reheating epoch in the early universe [10], but that can be circumvented [11-13]. Axinos are restricted to being cold if they are to be a DM candidate $[14,15]$.

The neutrino was originally considered as a DM candidate, but the neutrino relic density is calculated to be less than 0.07 due to constraints on the mass limit [16]. This is too low

for the DM relic density of about 0.118 (see Chapter 1). Sterile neutrinos were proposed as a DM candidate in Ref. [17]. The possible light mass also rules out the sterile neutrino, $O(10 \mathrm{eV})$, but if there is a small lepton asymmetry then a cold sterile neutrino DM may be possible [18]. 
Other models include axions, brane world DM, charged massive particles, cryptons, D-matter, DM from little Higgs models, heavy fourth generation neutrinos, Kahuza-Hlein states, mirror particles, Q-balls, self interacting DM, super heavy DM, superweakly interacting DM, etc. For more information on these models follow references in Ref. [4].

\subsection{LIGHT DARK MATTER THEORY}

Another DM theory to add to the possible list of explanations is called light dark matter (LDM) $[19,20]$. LDM was developed to explain the observed $511 \mathrm{keV}$ excess the INTEGRAL satellite observed from the galactic bulge [21, 22]. The WIMP model assumes the mediator between the dark sector and the SM $(V)$ to be the Z-boson. LDM is the simplest extension to this model where the mass of the mediator $m_{V}$ is unknown and the coupling between the dark sector and SM is not necessarily in the weak scale. Models were proposed and then used to show that current neutrino detectors should be sensitive in a region of the LDM parameter space [23-26].

LDM is a spontaneously broken theory, e.g. $U(1)$, that is not limited by the LeeWeinberg bound [27]. Because the DM annihilation cross section is dependent on $m_{\chi}^{2} / m_{V}^{4}$ and the Lee-Weinberg bound assumes $V$ is the Z-boson, the bound forbids a massive particle to satisfy the observed DM relic density to be less than $O(\mathrm{GeV})$. LDM has no assumption on the mass of the mediator, therefore, the dark matter particle can have any mass as long as the ratio $m_{\chi}^{2} / m_{V}^{4}$ does not get too small. The discussion in this section will only consider the vector mediator, a.k.a. vector portal, and will follow the discussion of Ref. [28].

With the vector mediator an extra spontaneously broken $U(1)^{\prime}$ symmetry is added to the SM using

$$
\begin{aligned}
\mathcal{L} & =\mathcal{L}_{\chi}-\frac{1}{4} F_{\mu \nu}^{\prime} F^{\prime \mu \nu}+\frac{1}{2} m_{V}^{2} V_{\mu} V^{\mu}-\frac{\epsilon}{2} F_{\mu \nu} F^{\prime \mu \nu}, \\
\mathcal{L}_{\chi} & =\left\{\begin{array}{ll}
\imath \bar{\chi}\left(\partial_{\mu}-\imath g^{\prime} V_{\mu}\right) \chi-m_{\chi} \bar{\chi} \chi & \text { Dirac fermion DM } \\
\left|\left(\partial_{\mu}-\imath g^{\prime} V_{\mu}\right) \chi\right|^{2}-m_{\chi}^{2}|\chi|^{2} & \text { Complex scalar DM }
\end{array} .\right.
\end{aligned}
$$


The extra Lagrangian $\mathcal{L}$ adds four free parameters to the SM: $\epsilon$ is the kinetic mixing angle between the dark and light sectors, $\alpha^{\prime}=g^{\prime 2} / 4 \pi$ is the square of the gauge coupling between the dark mediator and particle, $m_{V}$, and $m_{\chi}$. Fig. 2.1 shows schematically the role of $\epsilon$ and $\alpha^{\prime}$. There is nothing limiting $\chi$ to be a fermion or a scalar particle, but for this paper

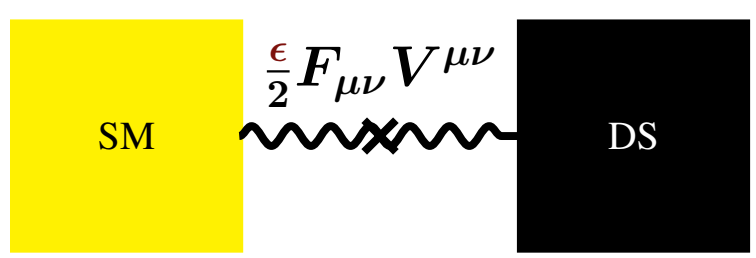

(a) Role of $\epsilon$

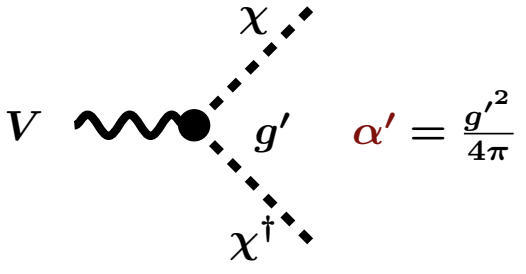

(b) Role of $\alpha^{\prime}$

Figure 2.1: Schematic view of the role of $\epsilon$ and $\alpha^{\prime}$ in LDM.

$\chi$ is assumed to be a complex scalar. The annihilation cross section, assuming $\epsilon$ is small, is given by

$$
\sigma v \sim \alpha^{\prime} \epsilon^{2} \alpha\left(\frac{m_{\chi}^{2}}{m_{V}^{4}}\right),
$$

where $\alpha$ is the electromagnetic fine structure constant, and the Feynman diagram is shown in Fig. 2.2.

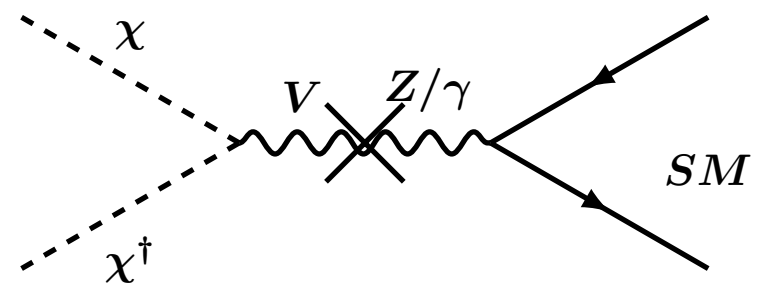

Figure 2.2: Feynman diagram showing two complex scalars annihilating into SM particles.

Defining

$$
Y=\alpha^{\prime} \epsilon^{2}\left(\frac{m_{\chi}}{m_{V}}\right)^{4},
$$

allows Eq. (2.5) to be written in terms of $Y$ which will be used as a convenient combined variable in Chapter 6. 


\subsubsection{MUON MAGNETIC MOMENT}

The current experimental measurements of the electron magnetic moment is consistent with theory, however, the muon shows a discrepancy greater than $3 \sigma$ [29-31]. LDM has the capability to reduce the discrepancy on the predicted and observed muon $g-2[32$, 33]. This is done by adding a 1-loop correction to the $\gamma$-fermion vertex, see Fig. 2.3. This

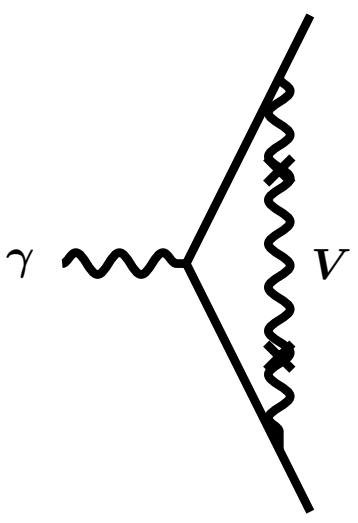

Figure 2.3: One-loop correction to the $\gamma$-fermion vertex in QED.

correction adjusts the electromagnetic fine structure constant, $\alpha$. One of the goals of this analysis was to test the parameter space where LDM solves the muon $g-2$ discrepancy.

\subsubsection{PRODUCTION}

For MiniBooNE, the production of $V$ in the beamline comes from two channels, $\pi^{0}, \eta$ decay-in-flight and proton bremsstrahlung. The Feynman diagrams are shown in Fig. 2.4.

\section{$\pi^{0}, \eta$ DECAY IN FLIGHT}

Decay-in-flight is assumed because the lifetime of the $\pi^{0}$ is $8.4 \times 10^{-8} \mathrm{~ns}$, the lifetime of $\eta$ is $5.02 \times 10^{-10} \mathrm{~ns}$, and the particles are neutral. Defining $\varphi=\pi^{0}, \eta$ the production channel for $\mathrm{DM}$ is

$$
\varphi \rightarrow \gamma+V \rightarrow \gamma+\chi^{\dagger}+\chi
$$




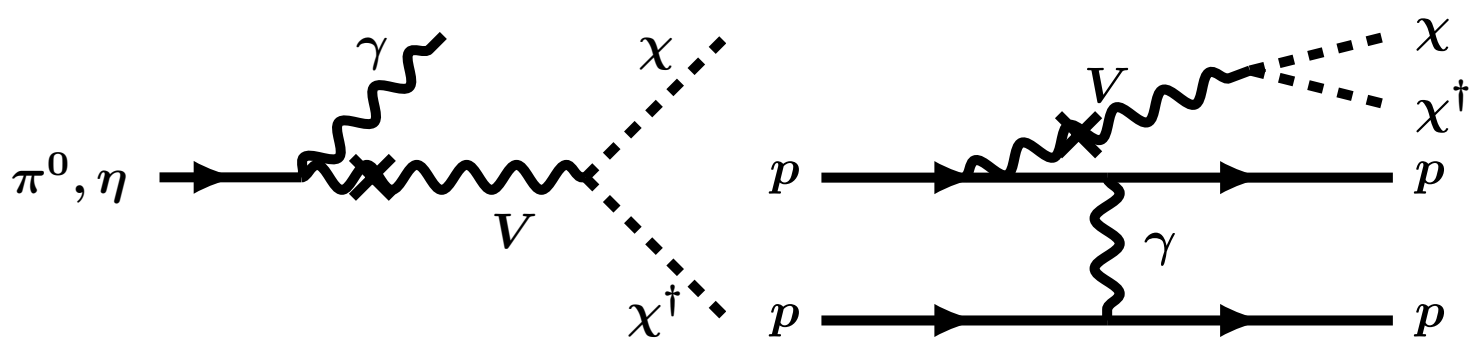

(a) Neutral Meson Decay

(b) Proton bremsstrahlung

Figure 2.4: Production of DM in a proton fixed-target/beam dump beamline.

The amplitude is then given by

$$
|\mathcal{M}|^{2}=\frac{k_{V}^{(1)} \alpha f\left(q^{2}, p \cdot k_{1}, p \cdot k_{2}\right)}{\pi f_{\varphi}^{2}\left[\left(q^{2}-m_{V}^{2}\right)^{2}+m_{V}^{2} \Gamma_{V}^{2}\right]},
$$

where $f=\left(q^{2}-4 m_{\chi}^{2}\right)\left(m_{\varphi}^{2}-q^{2}\right)^{2}-4 q^{2}\left(p \cdot k_{1}-p \cdot k_{2}\right)^{2}$ and $k_{V}^{(n)}=\epsilon^{2} \alpha\left(\alpha^{\prime}\right)^{n}$. In Eq. (2.6) $p$ is the photon momentum, $q$ is the momentum of $V, k_{1,2}$ are the dark sector particles' momentum in the final state where $q=k_{1}+k_{2}$, and $\Gamma_{V}$ is the decay width of $V$. No form factor is included, though one can be added to account for the virtuality dependence.

\section{PROTON BREMSSTRAHLUNG}

The other relevant production channel for MiniBooNE is through proton bremsstrahlung

$$
p+N \rightarrow p+N+V
$$

Although the total production rate through proton bremsstrahlung is lower than DM produced from $\pi^{0}, \eta$ decay, the angular spread is less, i.e. forward-peaked, thus contributing to more DM particles crossing the MiniBooNE detector. DM production through proton bremsstrahlung is still predicted to be uniform in MiniBooNE. The production rate of the number of $V$ is dependent upon: (i) $z$ the fraction of the beam momentum carried by the produced $V$, (ii) $p_{\perp}$ the perpendicular momentum of $V$ to the beam momentum, (iii) $m_{V}$, (iv) $\epsilon,(\mathrm{v}) \alpha^{\prime}$, and (vi) the form factor used. Vector particle mixing, e.g. $\rho \rightarrow V$, can 
be included in the same model as proton bremsstrahlung using a timelike form factor, $F_{1, N}\left(q^{2}\right)$ [28]. For this paper, the timelike form factor is used, therefore, DM production through bremsstrahlung includes vector particle mixing in the scope of this analysis. Ref. [28] shows the differential $V$ production rate as a function of $z$ and $p_{\perp}$ with the assumption

$$
E_{p}, E_{V}, E_{p}-E_{v} \gg m_{p}, m_{V},\left|p_{\perp}\right|
$$

where $E_{p}=P+m_{p}^{2} /(2 P), P$ is the momentum of the incident proton, and $E_{V}=z P+$ $\left(p_{\perp}^{2}+m_{V}^{2}\right) /(2 P z)[28]$. For this experiment $z \in[0.3,0.7]$ and $\left|p_{\perp}\right|<0.2 \mathrm{GeV}$.

\subsubsection{INTERACTION}

For this analysis, the only interaction considered is neutral-current elastic scattering of DM with either free or bound nucleons. The Feynman diagrams are shown in Fig. 2.5. The left diagram in Fig. 2.5 is the same as the right except the right includes nuclear

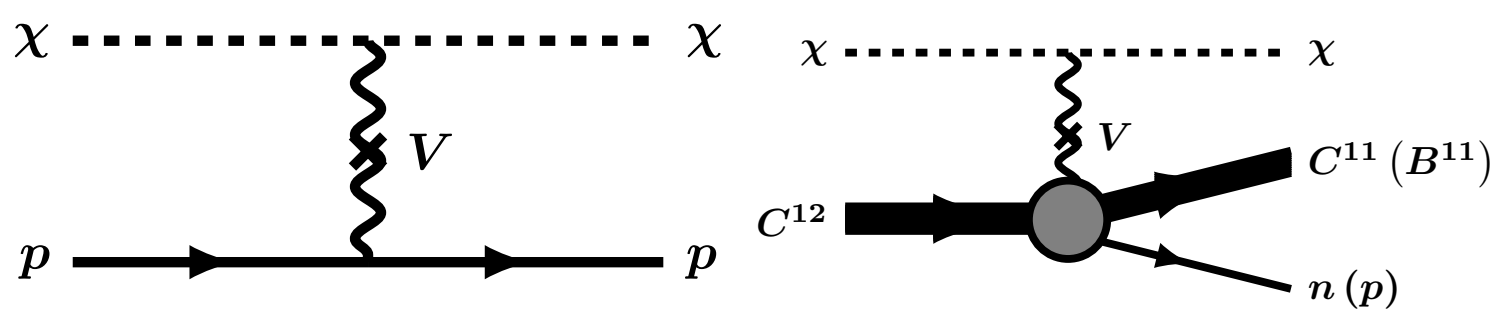

Figure 2.5: Interaction channels in LDM considered in this analysis.

effects for nucleons bound in carbon. The LDM theory developed to date only considers interactions with free nucleons. Applying the nuclear effects for nucleons bound in carbon will be discussed in Sec. 6.1.

The DM nucleon scattering cross section on free nucleons has the form

$$
\frac{d \sigma_{\chi N}}{d E_{\chi}}=4 \pi k_{V}^{(1)} Q_{N}^{2} G_{D}\left(Q^{2}\right) \frac{2 m_{N} E E_{\chi}-m_{\chi}^{2}\left(E-E_{\chi}\right)}{\left(E^{2}-m_{\chi}^{2}\right)\left(m_{V}^{2}+Q^{2}\right)^{2}}+\ldots
$$

where $Q_{N}$ is the nucleon electric charge, $E_{\chi}$ is the recoil DM energy, $Q^{2}=2 m_{N}\left(E-E_{\chi}\right)$ 
is the four momentum transfer with $E$ being the energy of the incoming DM particle, and $G_{D}\left(Q^{2}\right)$ is the Sachs form-factor, $G_{D}\left(Q^{2}\right)=1 /\left(1+Q^{2} / M^{2}\right)^{2}$, where $M=0.843 \mathrm{GeV}$. The next leading term includes dipole form factors and are included in the simulations described in Sec. 6.1 but are not shown for simplicity.

\subsection{EXPERIMENTAL SEARCH}

The discussion of experimental searches for DM is intertwined with specific types of DM. The discussion here focuses on direct search experiments.

\subsubsection{DARK MATTER DIRECT DETECTORS}

Many experiments have tried to detect DM in our solar system directly, that is, observation of DM interaction with particles in a purpose-built detector. Fig. 2.6 gives the best limits to date. From Fig. 2.6a the low mass sensitivity of the direct DM detectors searching for

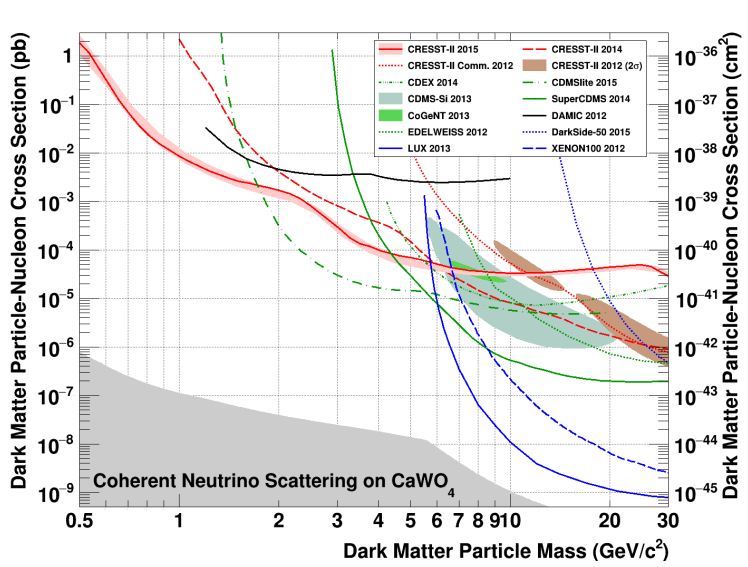

(a) Low Mass Limit [34]

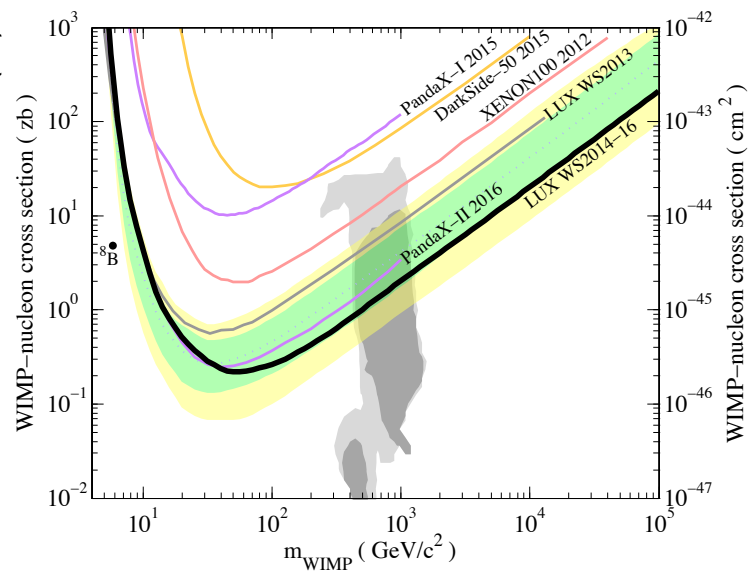

(b) Overall Limit [35]

Figure 2.6: Current best limits in direct DM WIMP searches.

WIMPs is $O(\mathrm{GeV})$. A way to lower the mass sensitivity is to search for boosted DM produced from an accelerator. The boosted DM will be able to exchange more energy in the detector, or decay into more energetic daughters making it easier to detect. Current 
collider experiments are able to search for missing energy, or the decay of the DM particle into standard model particles $[36,37]$. Fixed-target experiments are also able to detect the DM directly.

\subsubsection{PREVIOUS BEAM DUMP EXPERIMENTS}

Fixed-target searches have been preformed for decades to search for weakly interacting particles. Tables 2.1 and 2.2 lists some previous/current/future experiments who place limits on LDM. Table 2.1 (Table 2.2) shows experiments on proton (electron) beamlines. The table is not exhaustive, but gives a good idea of the variation of experiments which are sensitive to LDM. The initial goal for all experiments in Tables 2.1 and 2.2, except APEX and the third MiniBooNE run, was to search for non-DM axions, neutrinos, or rare decays. The limits from these experiments shown for LDM come from reinterpretation of the experiment's data; references for the reinterpretations are given in Tables 2.1 and 2.2 when applicable.

The APEX, Heavy Photon Search (HPS), and DarkLight experiments are currently being developed to search for $V$ produced in the electron beam at Jefferson National Laboratory (JLab). The signature of $V$ would be the standard model daughter products. SeaQuest at Fermi National Accelerator Laboratory (FNAL), is planning on doing a dedicated run to search for $V \rightarrow \mu^{-} \mu^{+}$[38]. Fig. 2.7 shows the comparison between current and proposed visible decay limits on $V$, a.k.a. $A^{\prime}$. Current limits on the invisible decay $2 m_{\chi}<m_{V}$ are given in Figs. 7.2 and 7.3.

MiniBooNE, with this analysis, is the first to do a dedicated proton beam dump run to set a limit on LDM. The following chapter explains the experimental setup of this run. 


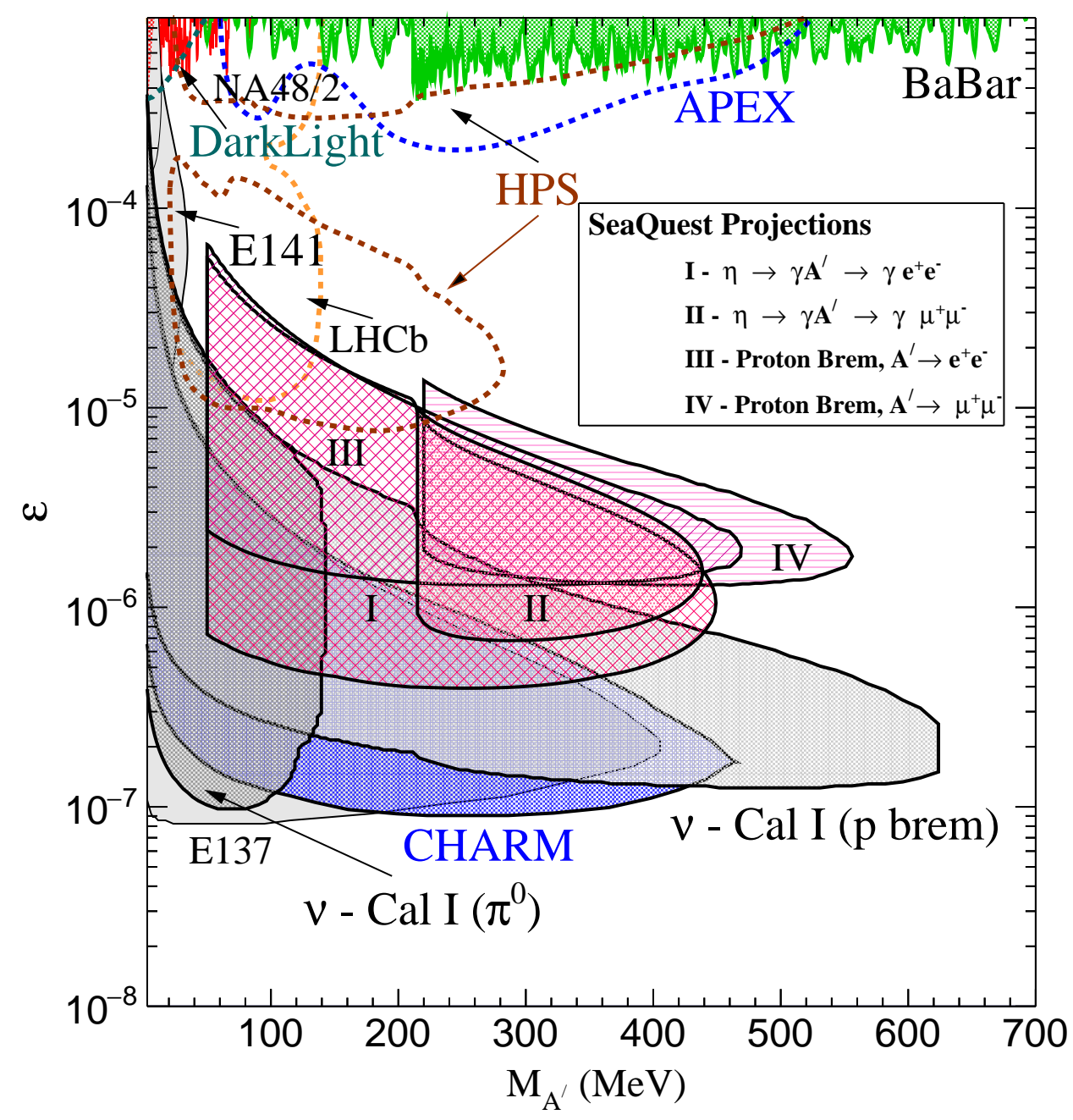

Figure 2.7: Visible decay limits. Dashed lines are predicted sensitivities for JLab and Large Hadron Collider (LHC) experiments. The Seaquest regions are also predicted. All other curves are current limits. From Ref. [38]. 


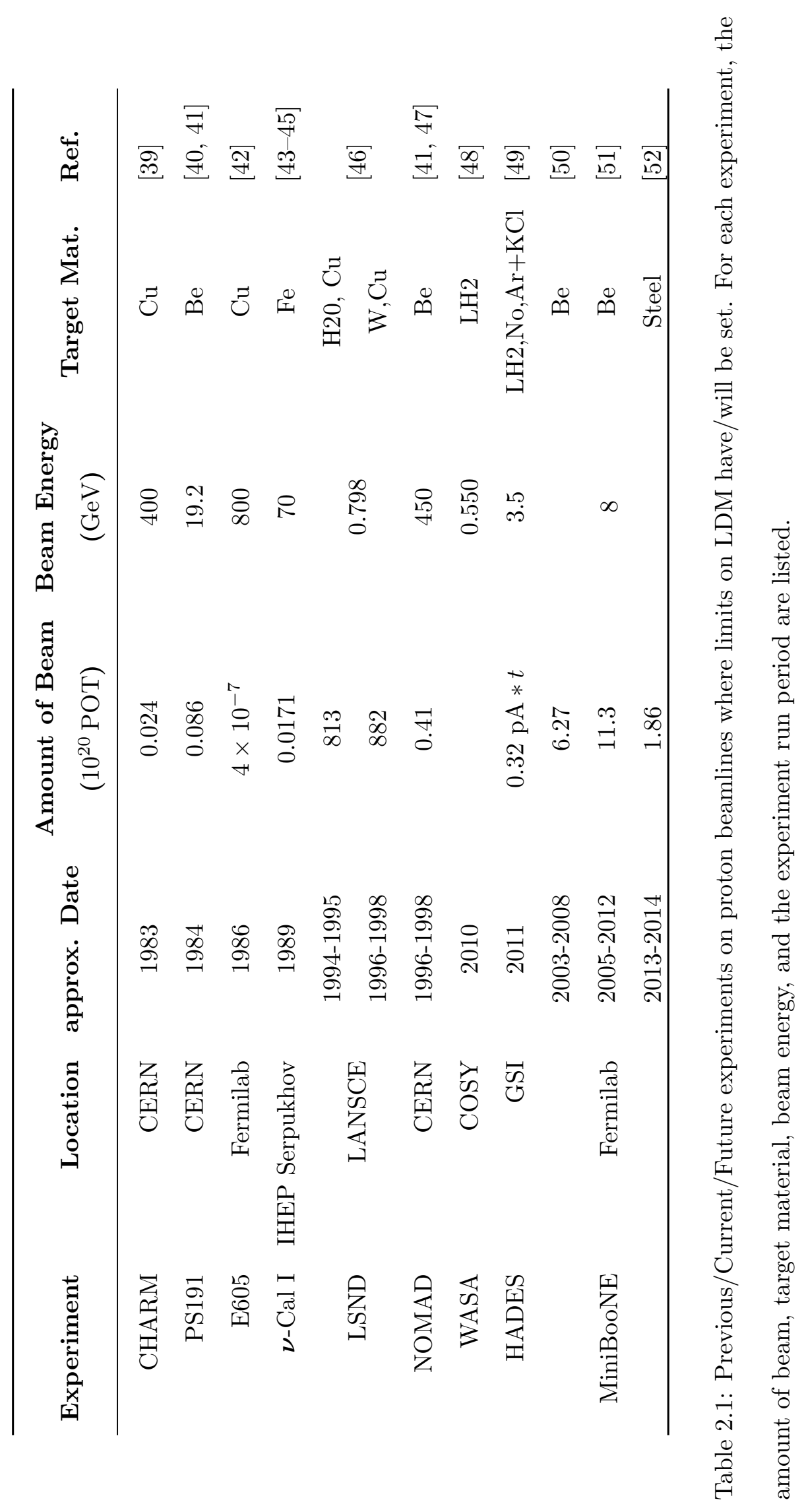




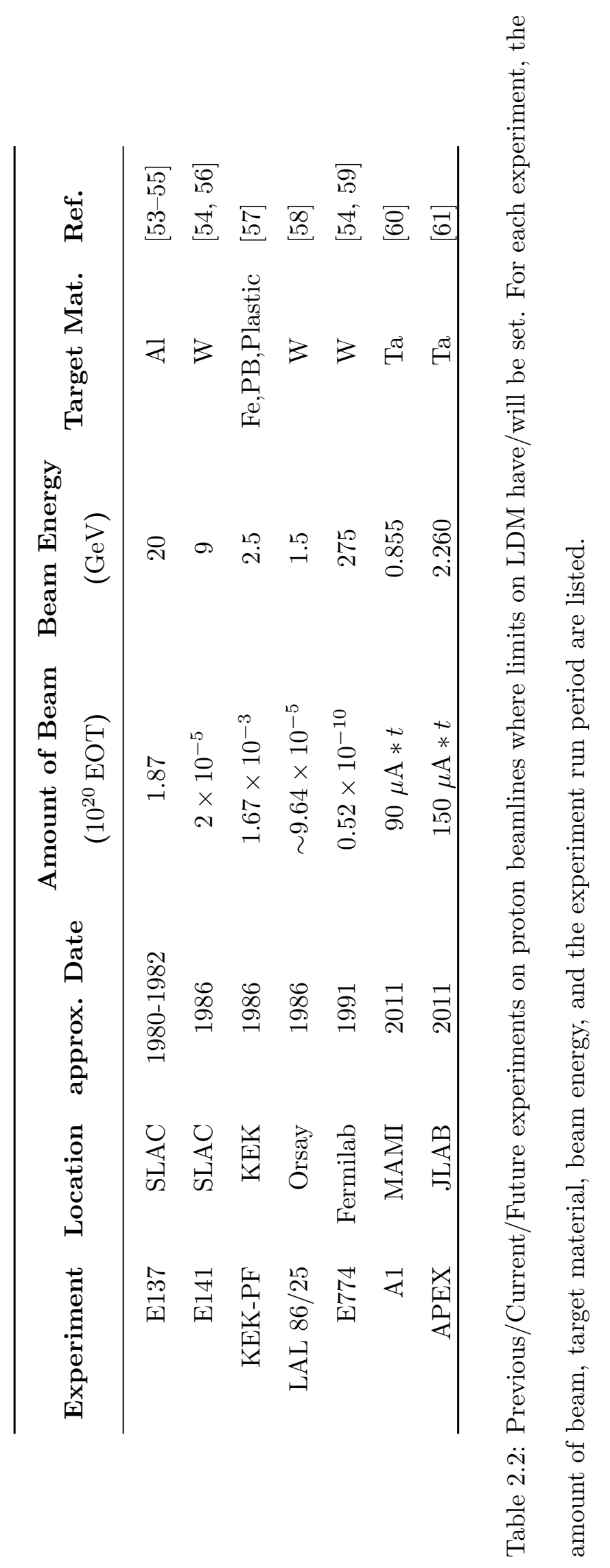




\section{CHAPTER 3}

\section{EXPERIMENTAL SETUP}

A search for dark matter (DM) produced in the Booster Neutrino Beamline (BNB) beamline at Fermi National Accelerator Laboratory (FNAL) was conducted using the MiniBooNE detector. MiniBooNE was chosen because it was already well understood after it had run for about a decade before doing the dedicated dark matter search. This chapter gives an overview of the BNB, the MiniBooNE detector, simulations, and track reconstruction. Details of the dark matter analysis follow in Chapters 4 to 6 .

\subsection{BOOSTER NEUTRINO BEAMLINE}

The MiniBooNE detector, discussed in Sec. 3.2 is located $541 \mathrm{~m}$ downstream of the BNB at FNAL, schematically shown in Fig. 3.1. The BNB delivers $8 \mathrm{GeV}$ protons to a beryllium target producing decay-in-flight neutrinos. The proton beam intensity ranges from $(1$ to 5$) \times 10^{12}$ protons per pulse $(\mathrm{ppp})$, where each pulse is $1.6 \mu \mathrm{s}$ long with a $53 \mathrm{MHz}$ microstructure. The microstructure of the beam consists of 2 ns wide "buckets" separated by $18 \mathrm{~ns}$.

Fig. 3.2 shows the $7.1 \mathrm{~cm}$-long, $0.51 \mathrm{~cm}$-radius beryllium target. The target is held in a beryllium shell by three beryllium fins creating a gap where air is circulated to cool the target. The target-shell structure is placed in an aluminum-alloy electromagnet, a.k.a. horn, to enhance either neutrino or anti-neutrino running [62]. A $214 \mathrm{~cm}$ concrete collimator 

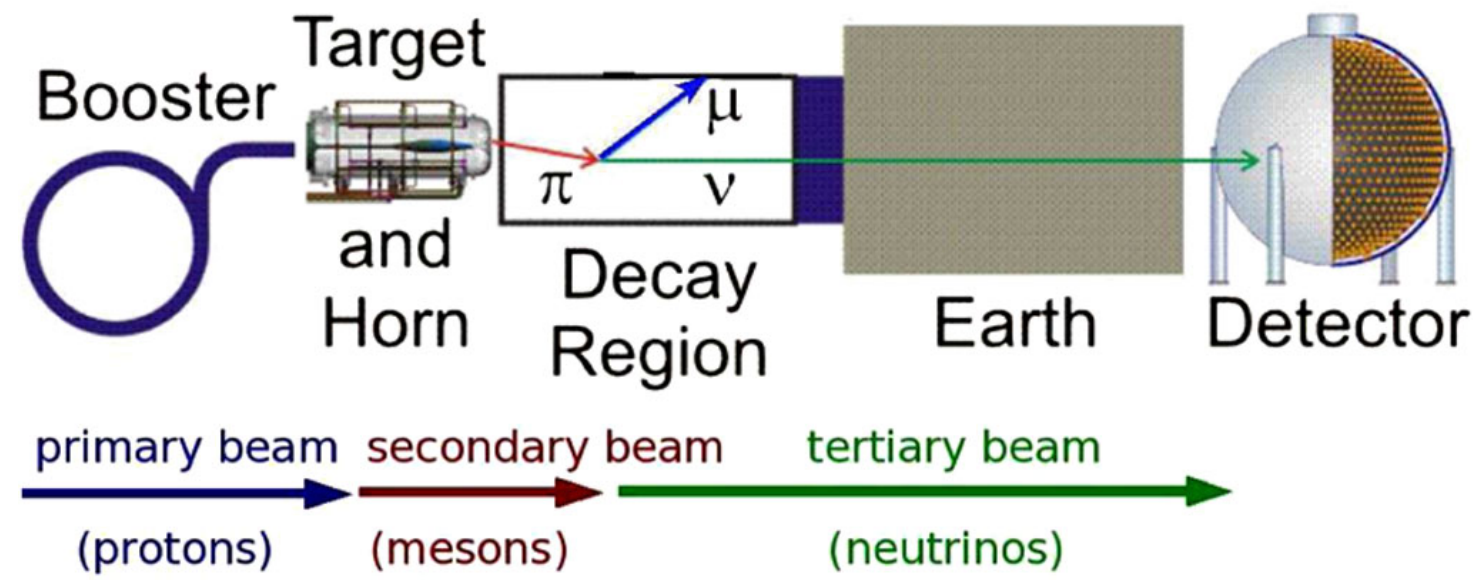

Figure 3.1: Schematic of how the neutrinos are produced at the BNB with respect to the MiniBooNE detector.

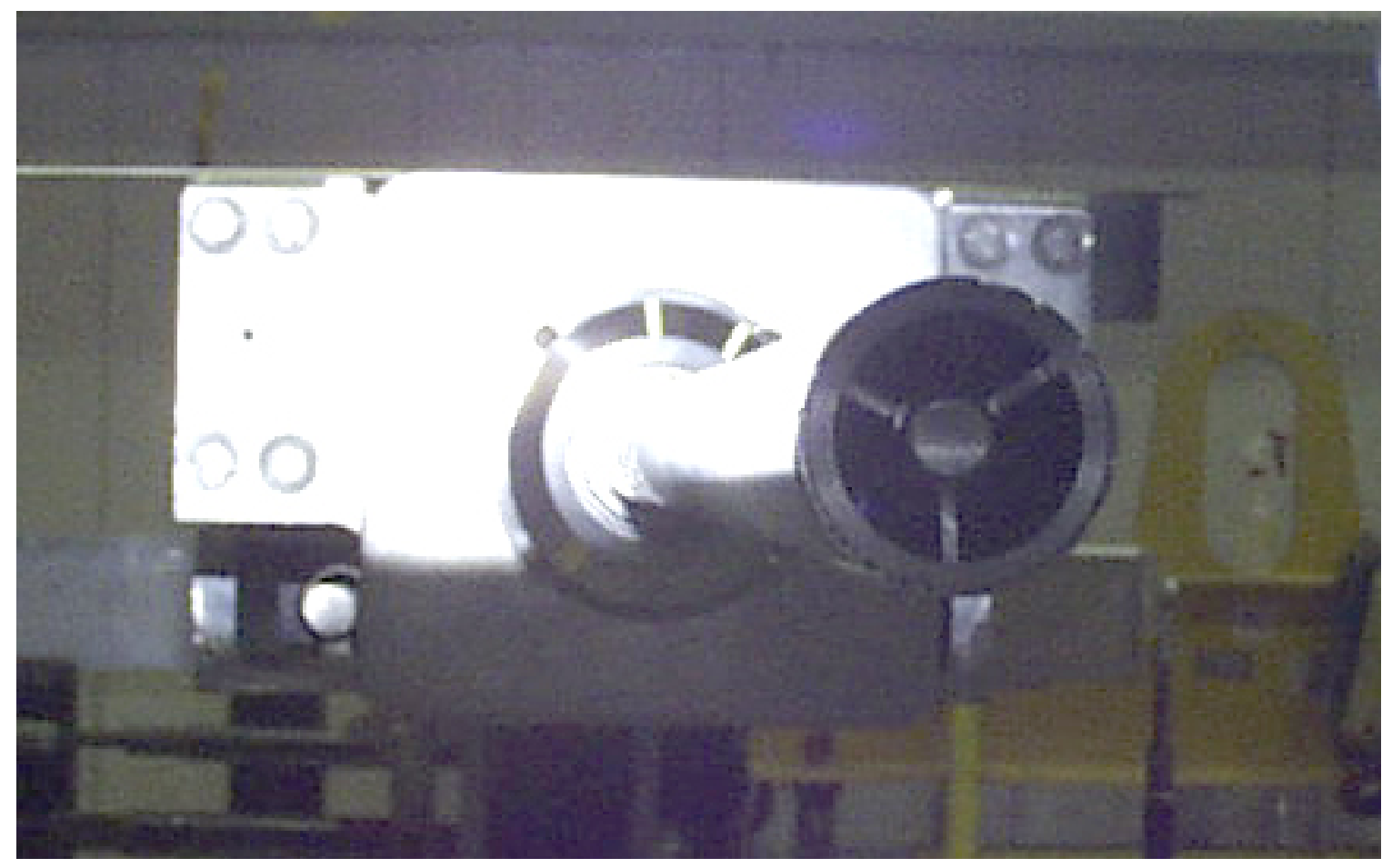

Figure 3.2: Picture of the BNB target 
is placed right after the horn to remove particles that would not contribute significantly to the neutrino flux. Downstream from the collimator there is $45 \mathrm{~m}$ of air, before the beam dump, acting as a decay pipe. The beam dump is composed of layers of steel and concrete.

Prior to the target, there consists a series of beam position monitors (BPMs) and loss monitors to determine the beam position during running operations. Before running, the beam is adjusted to find the target with respect to the BPMs. Fig. 3.3 is the output of a target scan, and shows that the BPM are calibrated well, although it looks like there is

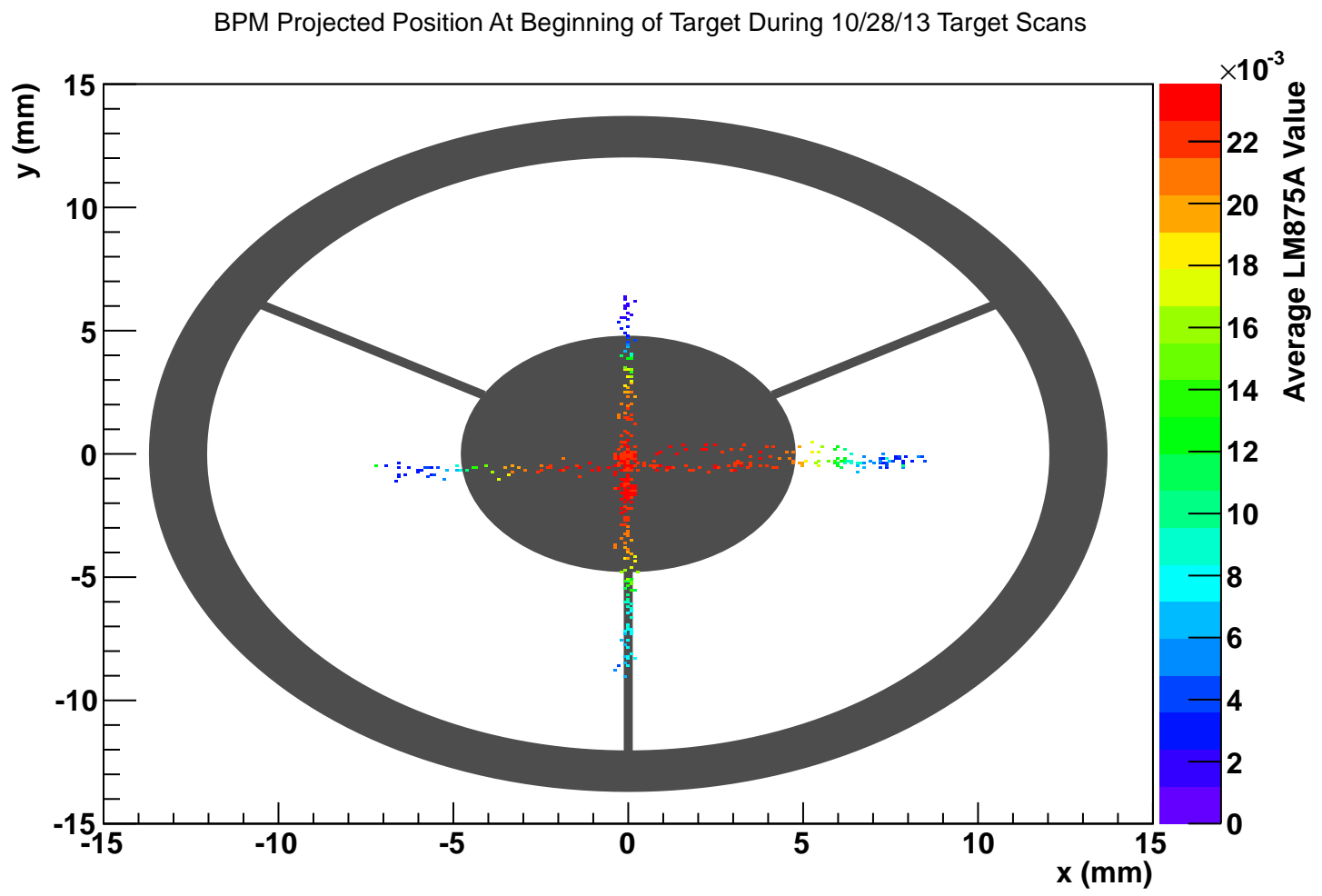

Figure 3.3: Results of a BNB target scan.

more loss in $+x$ than in $-x$. This is due to either the target not being exactly parallel with the beam or some material downstream of the target not aligned. The distinction cannot be made from this data.

There are three sets of multiwire detectors upstream of the target to have more precise proton bunch projection information to be used off-line. MWTGT is placed about $64 \mathrm{~cm}$ in- 
front of the face of the target and consists of 24 tungsten wires in each direction, with a radius of $1.5 \mathrm{mil}$ and separated by $0.5 \mathrm{~mm}$. The wires are attached to a $80 \mathrm{mil}$ thick $\mathrm{Al}_{2} \mathrm{O}_{3}$ ceramic disk with an inner radius of 625 mil. Just before and right after the wires is an aluminum foil that has an inner radius of 250 mil and is 10 mil thick. MWTGT was not in operation during most of the MiniBooNE runs but the beam does interact with the material. MW876 is placed around $440 \mathrm{~m}$ upstream of the face of the target and MW875 is about $1 \mathrm{~m}$ upstream of MW876. They consists of the same tungsten wires used in MWTGT but are place $1 \mathrm{~mm}$ apart. There is also a foil before and after the wires, but the beam does not interact with it. MW875 and MW876 were upgraded before the 2013-2014 run period.

A detailed study of the neutrino and anti-neutrino modes, labeled $\nu$-Mode and $\bar{\nu}$-Mode respectively, was published in Ref. [62]. BooNEG4BEAM is a GEANT4-based simulation [63] package where the cross sections for $\pi^{ \pm}$and $K^{ \pm}$were tuned for beryllium and aluminum based on Harp [64] and BNL E910 data [65]. BooNEG4BEAM simulates the particles that are generated when protons travel down the BNB target hall, decay pipe, and beam dump. To save computation time the results from BooNEG4BEAM are passed through BooBeAmNT to redecay each neutrino that got generated $N$ times to calculate the flux in units of $\nu /$ protons on target (POT) that passes the MiniBooNE cross sectional area.

The cross sections used in BooNEG4BEAM are parameterized with the Sanford-Wang distribution $(\mathrm{SW})$ for a given meson [66]

$$
\frac{d^{2} \sigma}{d p d \Omega}(p, \theta)=c_{1} p^{c_{2}}\left(1-\frac{p}{p_{B}-c_{9}}\right) \exp \left(-c_{3} \frac{p^{c_{4}}}{p_{B}^{c_{5}}}-c_{6} \theta\left(p-c_{7} p_{B} \cos ^{c_{8}} \theta\right)\right)
$$

where the double differential cross section is calculated with respect to the momentum $p$ (in $\mathrm{GeV} / \mathrm{c}$ ) and angle $\theta$ (in radians) of the meson. The angle is with respect to the incident proton. $p_{B}$ is the incident proton momentum in $\mathrm{GeV} / \mathrm{c}$ and $c_{1} \ldots c_{9}$ are fit parameters, where $c_{9}$ is not used for the pion distributions. Table 3.1 gives the results of the fit parameters for both $\pi^{+}$and $\pi^{-}$. The DM generator discussed in Sec. 6.1 defaults to using the 


\begin{tabular}{cccccccccc}
\hline Meson & $c_{1}$ & $c_{2}$ & $c_{3}$ & $c_{4}$ & $c_{5}$ & $c_{6}$ & $c_{7}$ & $c_{8}$ & $c_{9}$ \\
\hline$\pi^{+}$ & 220.7 & 1.080 & 1.000 & 1.978 & 1.32 & 5.572 & 0.0868 & 9.686 & 1.000 \\
$\pi^{-}$ & 213.7 & 0.9379 & 5.4545 & 1.210 & 1.284 & 4.781 & 0.07338 & 8.329 & 1.000 \\
\hline
\end{tabular}

Table 3.1: The $\pi^{+}$and $\pi^{-}$SW parameters $c_{1-9}$ used in the BooNEG4BEAm simulation package. Copied from Ref. [62]

average of the SW $\pi^{+}$and SW $\pi^{-}$distributions to simulate the $\pi^{0}$ distribution. Fig. 3.4 shows the $\nu$-Mode total flux $\Phi_{\nu}$ as a function of the true neutrino energy $E_{\nu}$. Integrating 0.2 to $3 \mathrm{GeV}$ gives $\Phi_{\nu}=5 \times 10^{-10} \nu \mathrm{POT}^{-1} \mathrm{~cm}^{-2}$ at the MiniBooNE location.

Table 3.2 gives different interaction/collision lengths for the various materials in the BNB. To reduce the neutrino background during the DM search, either the protons need

\begin{tabular}{rllllr}
\hline Interaction & $\mathrm{Be}$ & $\mathrm{Al}$ & $\mathrm{Fe}$ & Air & Concrete \\
\hline Nuclear Collision & 0.2993 & 0.2582 & 0.1037 & 508.8 & 0.2832 \\
Nuclear Interaction & 0.4210 & 0.3970 & 0.1677 & 747.7 & 0.4239 \\
Pion Collision & 0.4460 & 0.3541 & 0.1359 & 734.8 & 0.3996 \\
Pion Interaction & 0.5947 & 0.5064 & 0.2042 & 1013 & 0.5592 \\
Radiation & 0.3528 & 0.08897 & 0.01757 & 303.9 & 0.1155 \\
\hline
\end{tabular}

Table 3.2: Interaction and Collision lengths for the various materials in the BNB. All values are given in meters [67].

to pass through air or a "thick" target of material more dense than beryllium. Therefore, for this search the beam was steered between one of the air gaps around the target with the horn turned off. The protons then traveled through air down to the $50 \mathrm{~m}$ beam dump. Any charged particle produced in the beam dump would interact before decaying lowering both the flux of neutrinos and the energy of the neutrinos that are produced. Data was taken in 2013-2014 in this mode, resulting in $1.86 \times 10^{20}$ POT compared to $6.46 \times 10^{20} \mathrm{POT}$ 


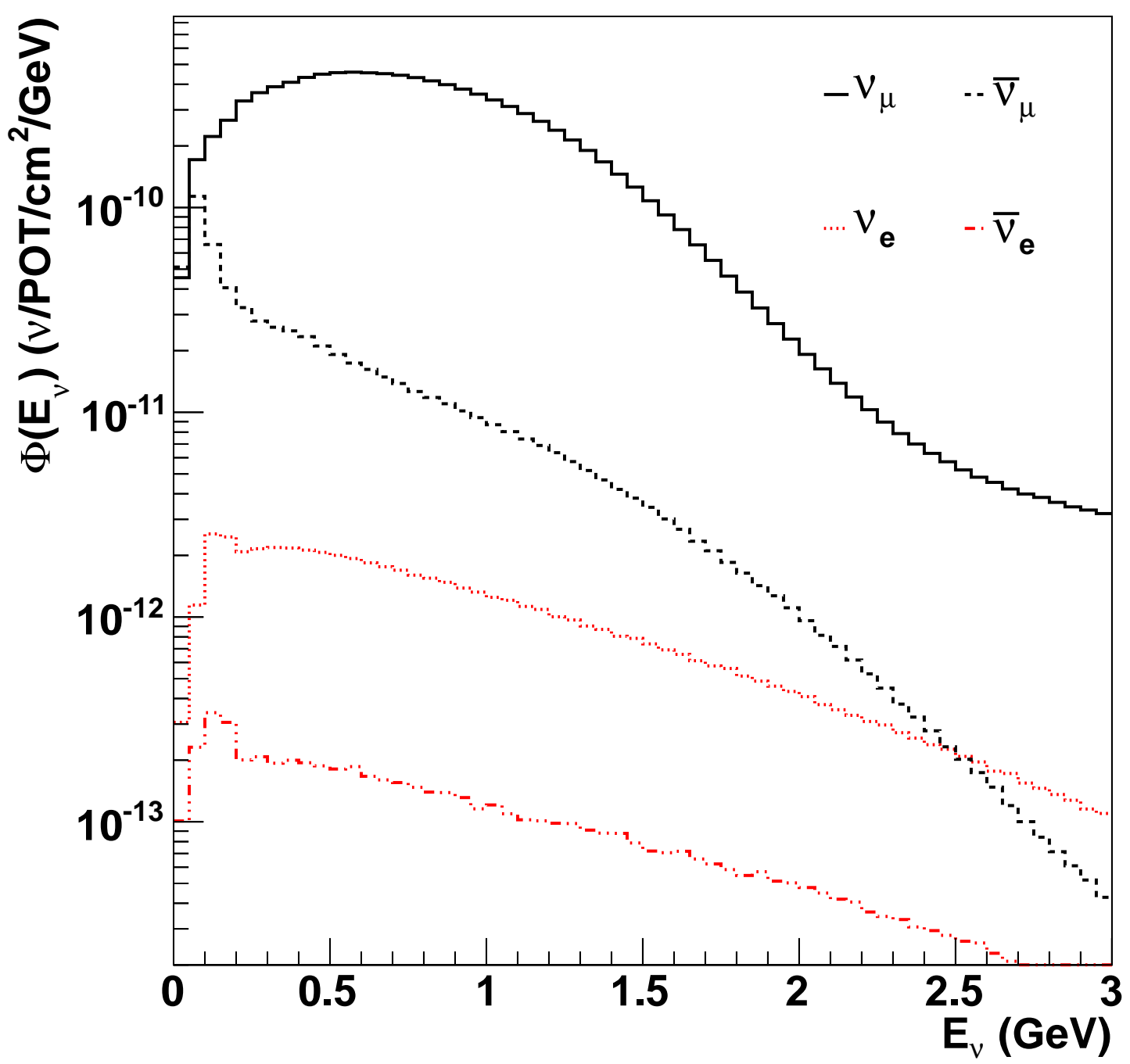

Figure 3.4: The $\nu$-Mode flux as a function of true $\nu$ energy predicted by the BooNEG4BEAM simulation package. 
obtained in $\nu$-Mode. The target of the run was the beam dump, but in this paper it is referred to as "off-target" because the beryllium target and horn were still in the beamline. Sec. 4.2 describes the development and study of the off-target flux.

\subsection{MINIBOONE DETECTOR}

The MiniBooNE detector, Fig. 3.5, is a mineral oil Cherenkov detector with a scintillation component [68]. The mineral oil sits inside a $610 \mathrm{~cm}$ radius sphere with two regions sepa-
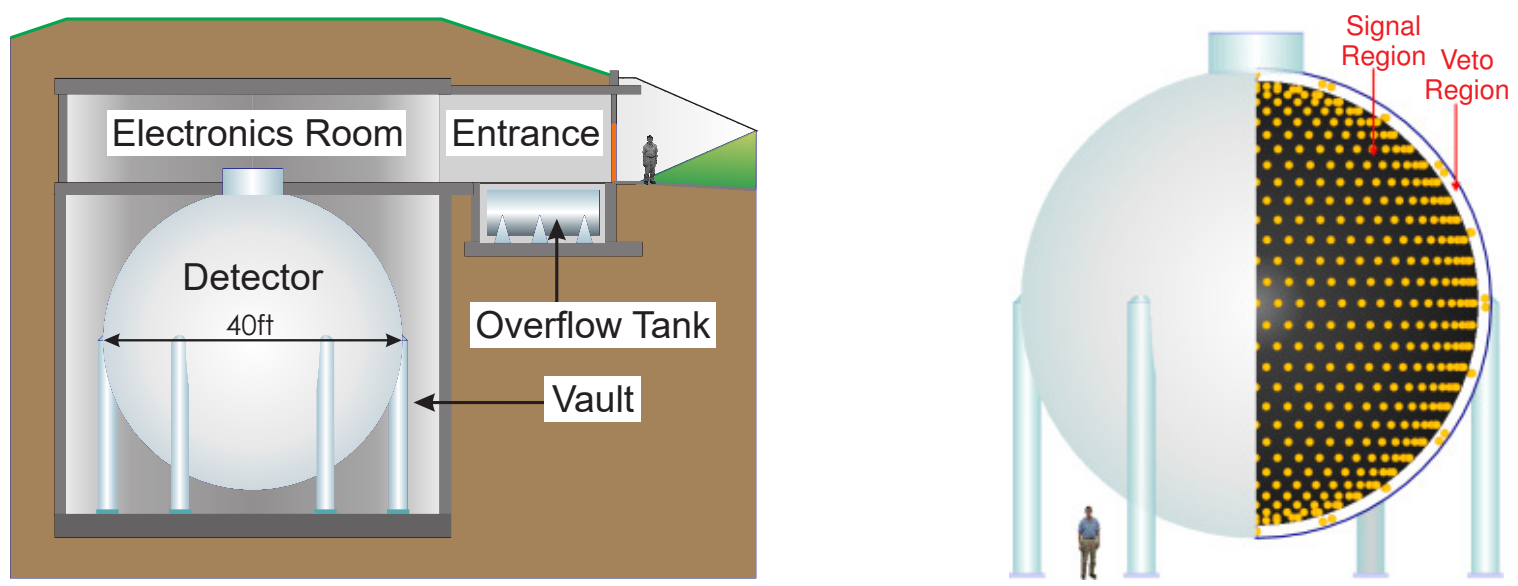

Figure 3.5: A schematic of the MiniBooNE detector showing (left) the cut-away drawing and (right) the location of the photomultiplier tubes as well as the separation between the signal and veto regions. Copied from Ref. [68]

rated by an optical barrier at $575 \mathrm{~cm}$. The inner, signal region consists of 1280 inward facing 8-inch photomultiplier tubes (PMTs) that have a total photocathode coverage of $11.3 \%$. The outer, veto region consists of 240 PMTs arranged in back-to-back pairs. The veto region thickness was chosen to best optimize signal region size and to reject beam-unrelated muons and beam-related neutrino-induced events outside the detector to greater than $99 \%$. The PMTs have an intrinsic time resolution of $\sim 1 \mathrm{~ns}$ and have a charge resolution of $15 \%$ at 1 photoelectron.

MiniBooNE is filled with 807 tons of Marcol-7 mineral oil $\left(\mathrm{CH}_{2}\right)$ with an index of re- 
fraction of $n=1.47$ and a density of $0.85 \mathrm{~g} \mathrm{~cm}^{-3}$. The PMTs have an index of refraction of $n \simeq 1.5$. Nitrogen is circulated constantly through the mineral oil to purge the detector of oxygen. This is important for light yield and structure longevity. Impurities in the mineral oil cause the scintillation light [68].

\subsubsection{DAQ AND TRIGGER SYSTEM}

The MiniBooNE data acquisition (DAQ) system uses 14 QT VME crates to record and analyze the detector and veto PMT hits which are recorded with a $10 \mathrm{MHz}$ sampling rate [68]. Charge and time information for each PMT hit is processed on eight-channel QT cards (16 per crate). If the preamp PMT signal exceeds about 0.1 photoelectron, the discriminator signal is activated digitizing for $200 \mathrm{~ns}$. The single photoelectron resolution is about 4 bits corresponding to a full scale of 20 to 30 photoelectrons. The preamplifier is continuously integrated with a capacitive circuit having a decay constant of about $700 \mathrm{~ns}$.

The charge measurement is set to the digitized value of at each $10 \mathrm{MHz}$ clock tick. A ramp voltage is generated using a fixed transistor ramp circuit triggered by the discriminator being activated. The voltage is digitized at $10 \mathrm{MHz}$ and quickly returns to baseline after two subsequent clock ticks. The time of the hit is determined by extrapolating two successive measurements of the ramp voltage to baseline. The analog-to-digital conversion happens independently of the trigger (see below).

Each digitized signal is stored in a buffer for approximately $200 \mu$ s for the trigger to use before the signal is overwritten. The data rate loss in normal running in less than 0.1\%. Each QT crate contains an integrator board that sums the total number of hits and the total charge the create accumulated for each clock tick. This information is sent to the triggering create and is used to create trigger definitions as described below. More information about the DAQ can be found in Ref. [68].

A complex trigger system was designed to create different trigger windows depending 
on the number of tank/veto hits as well as outside signals. Table 3.3 gives the condition and reason for each of the comparators, and external trigger settings.

\begin{tabular}{llll}
\hline Input & Name & PMT hits & Purpose \\
\hline External 1 & BEAM & n/a & Beam to MiniBooNE \\
External 2 & RANDOM & n/a & Strobe, NuMI, Debuncher \\
External 3 & CALIB & n/a & Calibration event \\
& & & \\
Comparator 1 & DET1 & \# tank hits $\geq 10$ & Activity Monitor \\
Comparator 2 & DET2 & \# tank hits $\geq 24$ & Michel electron \\
Comparator 3 & DET3 & \# tank hits $\geq 200$ & High-energy neutrino \\
Comparator 4 & DET4 & \# tank hits $\geq 100$ & Neutrino candidate \\
Comparator 5 & DET5 & \# tank hits $\geq 60$ & Supernova $\nu$ candidate \\
Comparator 6 & VET01 & \# veto hits $\geq 6$ & Cosmic veto \\
Comparator 7 & VET02 & \# veto hits $\geq 4$ & Cosmic activity \\
\hline
\end{tabular}

Table 3.3: The three external and seven comparator trigger settings. Copied from Ref. [68].

If any of the external triggers, DET1, or VET01 are true a word containing the state of all comparators and external triggers at the $100 \mathrm{~ns}$ clock tick is stored in the trigger FIFO register (2048 words deep). The words are composed in pseudo-realtime to select events for readout. For each event that is stored to disk the previous 90 trigger words are stored regardless of time. This information will be used to reduce the amount of beam-unrelated background (BUB), discussed in Sec. 4.1.1.

Various triggers are defined depending on the trigger word or group of words that are built. Two of the external triggers, BEAM and STROBE, both open a 19.2 $\mu$ s DAQ window with no condition on the detector activity. The BEAM trigger is defined as seeing the "beam to MiniBooNE" accelerator signal while the STROBE trigger comes from a "random" clock. In the BEAM trigger the beam arrives about $5 \mu$ s into the trigger; this provides a measurement of the BUB using the pre-beam information. For on-target running, BUB was a small 
percentage of the background $[50,51,69,70]$. Chapter 4 explains how lowering the produced neutrinos affects the percentage of BUB in this analysis. See Ref. [68] for more information on the trigger.

\subsubsection{MEASURING BEAM UNRELATED BACKGROUNDS}

Beam unrelated backgrounds come from cosmics and radioactive material in the ground surrounding the detector. The purpose of the STROBE trigger is to measure the BUB that would be seen underneath the beam signal. It is important for STROBE data to be collected coincidentally with BEAM data so that the detector conditions are the same in the two data sets. For on-target running the STROBE was set to $2.01 \mathrm{~Hz}$ while off-target used a rate of 10 to $15 \mathrm{~Hz}$. The increase in rate was necessary because the BUB was going to be a larger percentage of events with the reduction of the neutrino flux and therefore a larger sample was needed to reduce the BUB statistical uncertainty contribution. BUB was also measured by using the pre-beam information as a check. In off-target mode, 4.5 times more STROBE triggers were analyzed than for $\nu$-Mode. Not all the $\nu$-Mode STROBE triggers were used in the analysis.

Each STROBE event's weight is the ratio of the number of BEAM triggers to the number of STROBE triggers. The BNB trigger that BEAM is based on comes from a clock that is independent of if there is beam or not. Sometimes the clock is left running although no beam is being sent down the beamline. The Intensity Frontier Database (IFDB) is used to find the fraction of triggers that did not contain beam. For off-target running this fraction was $7.9 \%$. IFDB is also used as a check in the number of beam triggers recorded in the MiniBooNE database. For off-target running the difference between the two databases was $0.4 \%$.

For $\nu$-Mode running IFDB was not up and running, so the pre-beam data was used as a check [71]. A large discrepancy was found between the ratio of pre-beam and the 

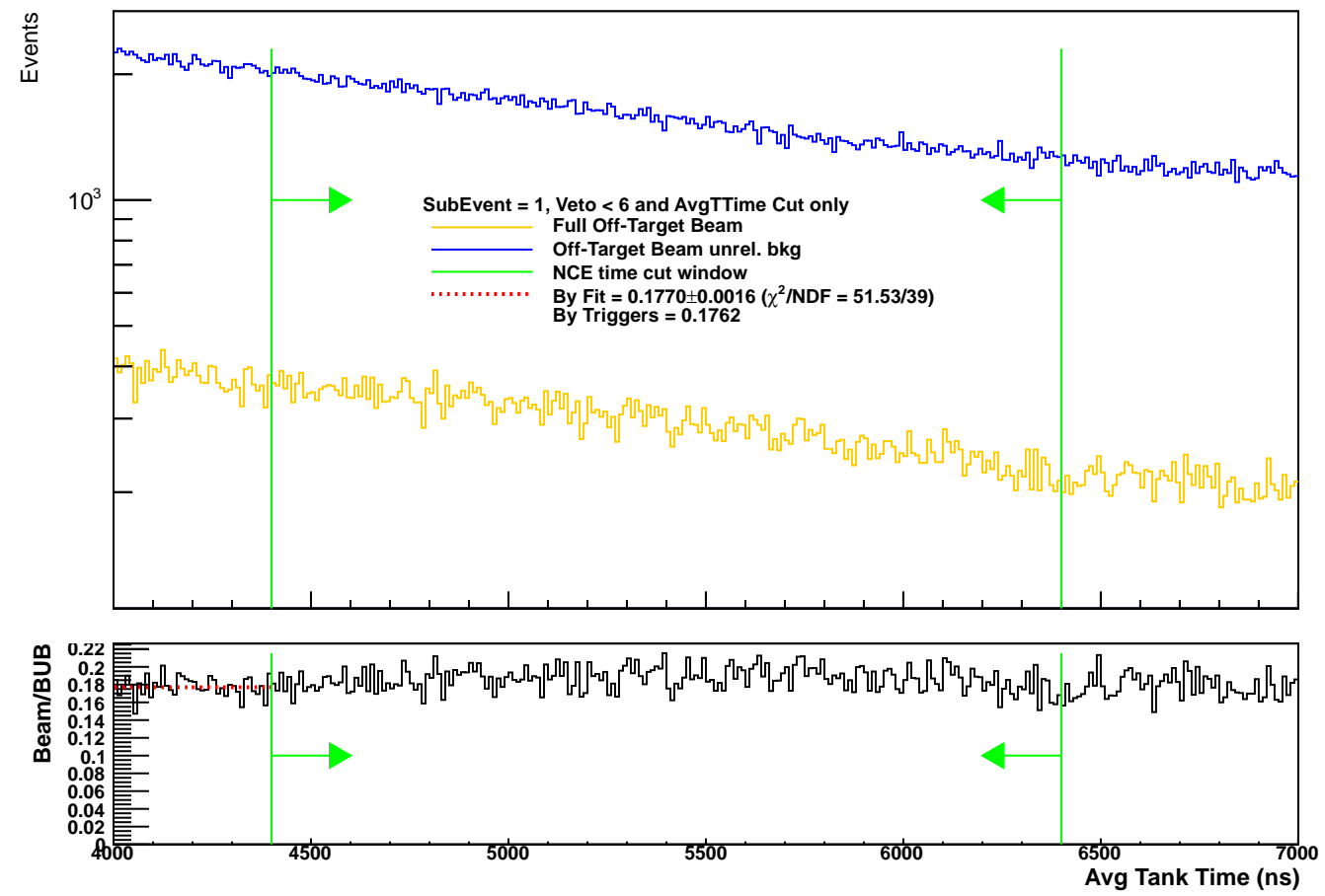

Figure 3.6: Checking the scaling of STROBE triggers to BEAM triggers. The blue line (top plot top line) is data from STROBE while the orange line (top plot bottom line) is data from BEAM. The axis is time in the DAQ window. The green vertical lines and arrows shows the beam time cut discussed in Sec. 4.1.1. The bottom plot is the ratio of BEAM to STROBE it is in good agreement with what one would expect after adjusting the number of BEAM triggers to account for empty triggers. 
ratio of triggers [71], so the uncertainty on the STROBE scaling was set to $100 \%$. This adds a systematic uncertainty of only $1.3 \%$ to the $\nu$-Mode neutral-current elastic (NCE) events due to the small percentage of BUB (see Table 4.8). The same check was done for off-target with the $7.9 \%$ correction and good agreement was found, see Fig. 3.6. The systematic uncertainty on the off-target STROBE scaling was set to $0.4 \%$ which adds a systematic uncertainty of $0.2 \%$ to the off-target NCE sample (see Table 4.8).

\subsubsection{DETECTOR SIMULATION}

The simulation (MC) of the beam related neutrino events was split into two, detector simulation (detMC) and dirt simulation (dirtMC). The only difference between detMC and dirtMC was the size of the volume the neutrinos were allowed to interact in; detMC (dirtMC) used a radius of $610.6 \mathrm{~cm}(14 \mathrm{~m})$. The $\mathrm{MC}$ was split into neutrino cross section modeling and particle transport/detector response.

\section{NEUTRINO CROSS SECTION MODEL}

The NUANCE V3 neutrino event generator [72] was used to simulate neutrino interactions in $\mathrm{CH}_{2}$. Refs. [50] and [69] describe the relevant simulation models for NCE and chargedcurrent quasielastic (CCQE) respectively. The relativistic Fermi gas model of Smith and Moniz [73] is used to describe both NCE and CCQE events on carbon, while LlewellynSmith was used for free hadrons [74]. For this paper NCE is defined as the incoming particle interacting with a single nucleon causing the nucleon to recoil. This is in contrast with Super-K which includes the detection of the de-excitation gammas from excited nucleons [75].

Final-state interactions of the nucleons in the carbon nucleus are included, which includes pion absorption. The axial form factor is assumed to be of dipole form with an axial mass $M_{A}$ and a Pauli-blocking parameter $\kappa$ is introduced as an extra degree of freedom to 
model the low- $Q^{2}$ events in MiniBooNE correctly.

Table 3.4 gives the parameters that were used to generate the $\nu$-Mode events. Ref. [69]

\begin{tabular}{lc}
\hline Parameter & Value \\
\hline$M_{A}$ for QE events on carbon & $1.2341 \mathrm{GeV}$ \\
Binding energy for carbon & $34.0 \mathrm{MeV}$ \\
Fermi momentum for carbon & $220.0 \mathrm{MeV}$ \\
$\Delta s$, the axial vector isoscalar term & 0.0 \\
$M_{A}$ for CC and NC single pion events & $1.1 \mathrm{GeV}$ \\
$M_{A}$ for CC and NC multiple pion events & $1.3 \mathrm{GeV}$ \\
Scale factor for NC coherent $\pi^{0}$ events & 1.302 \\
Scale factor for NC and CC $\Delta$ radiative events & 1.00 \\
Scale factor for deep inelastic scattering events & 1.00 \\
Pauli blocking scale factor, $\kappa$ & 1.0220 \\
$M_{A}$ for for CC single coherent events (not coherent $\left.\mathrm{NC} \pi^{0}\right)$ & $1.030 \mathrm{GeV}$ \\
Scale factor for NC resonant $\pi^{0}$ events & 1.00 \\
$M_{A}$ for QE events on hydrogen & $1.13 \mathrm{GeV}$ \\
\hline
\end{tabular}

Table 3.4: The NUANCE parameters used to generate the $\nu$-Mode detMC events. QE $=$ quasi-elastic, $\mathrm{CC}=$ charge-current, $\mathrm{NC}=$ neutral-current. Copied from Ref. [76]

measured the effective axial mass $M_{A}^{\text {eff }}$ and $\kappa$ to be $1.35 \mathrm{GeV}$ and 1.007 , and reported an extra 1.08 scale-factor to match simulations with $\nu$-Mode data. For this analysis, all detector and dirt simulated events were reweighted to these updated measured values, while only the CCQE distributions includes the scale-factor. The overall scaling is allowed to float (see Sec. 5.2).

Kinematic equations are used to determine $Q_{Q E}^{2}$, the reconstructed four momentum transfer for both NCE and CCQE events under the assumption the target nucleus is at rest. These equations are given in Eqs. (4.1) and (4.2) for CCQE and NCE events. 


\section{PARTICLE TRANSPORT/DETECTOR RESPONSE}

A GEANT3 simulation [77] was developed to simulate the particle transport in the Mini-

BooNE detector. Fig. 3.7 shows the detector geometry modeled in the detector simulation.

Light can be generated through ionization, Cherenkov radiation, scintillation, fluorence, or

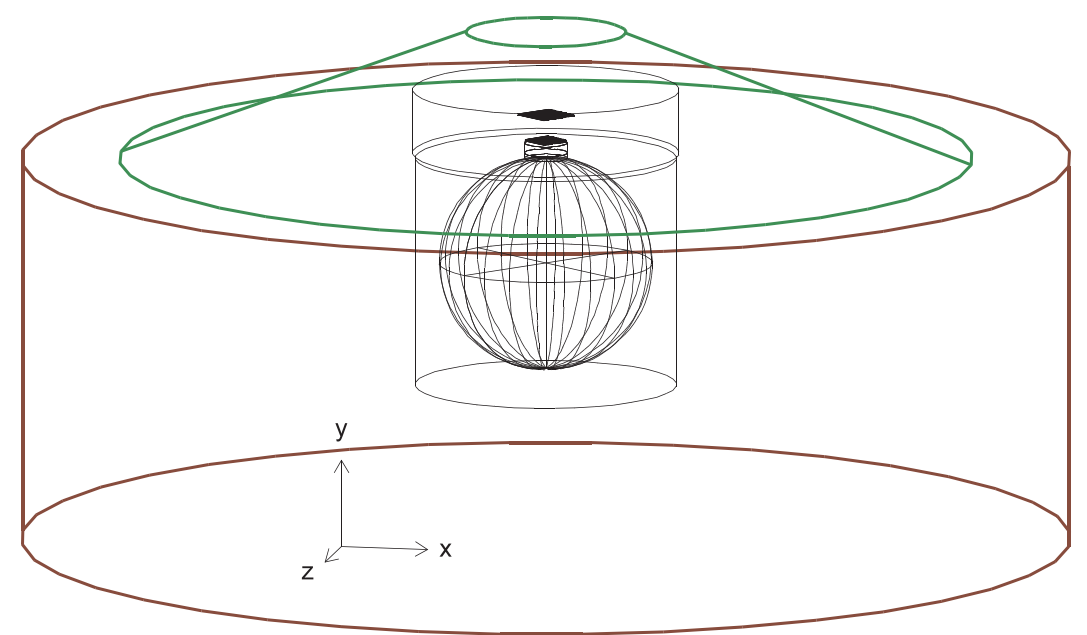

Figure 3.7: The geometry domain of the MiniBooNE GEANT3 simulation. The dirt region and overburden are indicated by the thick-lined cylinder and the conical frustum, the thin-lined cylinders indicate the electronics room and vault, the sphere and the top-hat determine the detector tank and the tophat. From Ref. [71]

scattering. Ionization occurs when a particle transfers enough energy to a bound electron causing it to become a free electron. Cherenkov radiation occurs when a particle's relative velocity to the speed of light $\beta$ is greater than $1 / n$. The kinetic energy of a particle required to produce Cherenkov light is

$$
T_{C h}=m\left(\frac{n}{\sqrt{n^{2}-1}}-1\right)
$$

where $m$ is the mass of the particle. Electrons have $T_{C h}^{e} \simeq 0.20 \mathrm{MeV}$ and protons have $T_{C h}^{p} \simeq 350 \mathrm{MeV}$. The primary light generation in MiniBooNE for electrons (protons) is Cherenkov (scintillation).

Scintillation light occurs when energy from the traveling particle excites a bound elec- 
tron to a higher level state. The de-excitation light from the electron is scintillation. The amount of scintillation light generated is proportional to the particle's ionization loss and quenching factor. Birks' law [78] relates the scintillation light yield to ionization energy loss per unit length

$$
\frac{d E_{s c i}}{d x}=\frac{d E_{i o n} / d x}{1+k_{B} \frac{d E_{i o n}}{d x}+k_{B}^{\prime}\left(\frac{d E_{i o n}}{d x}\right)^{2}}
$$

where $k_{B}=0.014 \mathrm{~g} \mathrm{~cm}^{2} \mathrm{MeV}^{-1}$ and $k_{B}^{\prime}=0$ were obtained empirically for MiniBooNE's mineral oil. While Cherenkov light is prompt scintillation light is delayed based on the lifetime of the electron in the excited state. For the MiniBooNE mineral oil the scintillation life time is $\sim 35$ ns [79].

Photons produced in the detector can interact in the detector while traveling to the PMTs. Scattering is the process when the photon gets absorbed and readmitted at the same wavelength but possibly a different direction. Fluorescence is the process when the admitted photon is a different frequency than the absorbed photon. Scattering, fluorescence, ionization and loss of photons from absorption, were measured and included in the MiniBooNE GEANT3 simulation [80, 81].

Digitization of the PMT signal was also simulated so the output from the simulations was in the same form as that from data. BUB data was overlayed on top of the simulations, based on analysis of the $\nu$-Mode STROBE trigger, to correctly model beam events that get cut because of coincident BUB events modifying the reconstructed charge/time of the neutrino event or causing there to be too many events in the DAQ window. The single BUB event rate changed from $4.24 \mathrm{kHz}$ in $\nu$-Mode to $4.40 \mathrm{kHz}$ in off-target.

\subsubsection{EVENT RECONSTRUCTION}

Not knowing a priori which type of production the event is, e.g. electron, muon, nucleon, different particle hypothesis tests were developed based on the topology for each event. For example, a high energy muon is expected to be a sharp Cherenkov ring while an electron 
will be a fuzzy Cherenkov ring. Most of the nucleon events have PMTs that contain charge and time consistent with scintillation light. The following describes the NCFitter developed to reconstruct proton recoil events in the detector. Discussion is based on Ref. [76, 79].

As previously stated, the proton has a Cherenkov threshold of $350 \mathrm{MeV}$. Most of the neutral-current elastic events seen in MiniBooNE are below this threshold, nonetheless $\mathrm{NC}$ Fitter uses both the scintillation and Cherenkov light to reconstruct the outgoing nucleon.

The predicted charge for a given $\mathrm{PMT}, \mu_{i}$ given track parameters $\mathbf{X}$ is given by

$$
\begin{aligned}
& \mu_{s c i}(\mathbf{X})=\epsilon \phi_{s c i}(E) \frac{\exp \left[-r / \lambda_{s c i}(R)\right]}{r^{2}} f(\cos \eta) F_{s c i}(E, \cos \theta, R) \operatorname{Cor} r(E, \cos \alpha) \\
& \mu_{c e r}(\mathbf{X})=\epsilon \phi_{c e r}(E) \frac{\exp \left(-r / \lambda_{c e r}\right)}{r^{2}} f(\cos \eta) F_{c e r}(E, \cos \theta, R) \operatorname{Corr}(E, \cos \alpha)
\end{aligned}
$$

where sci stands for scintillation and cer stands for Cherenkov. The rest of the parameters are as follows:

- $\mathbf{X}=\left(x_{0}, y_{0}, z_{0}, t_{0}, \theta_{0}, \phi_{0}, E_{0}\right)$ as shown in Fig. 3.8.

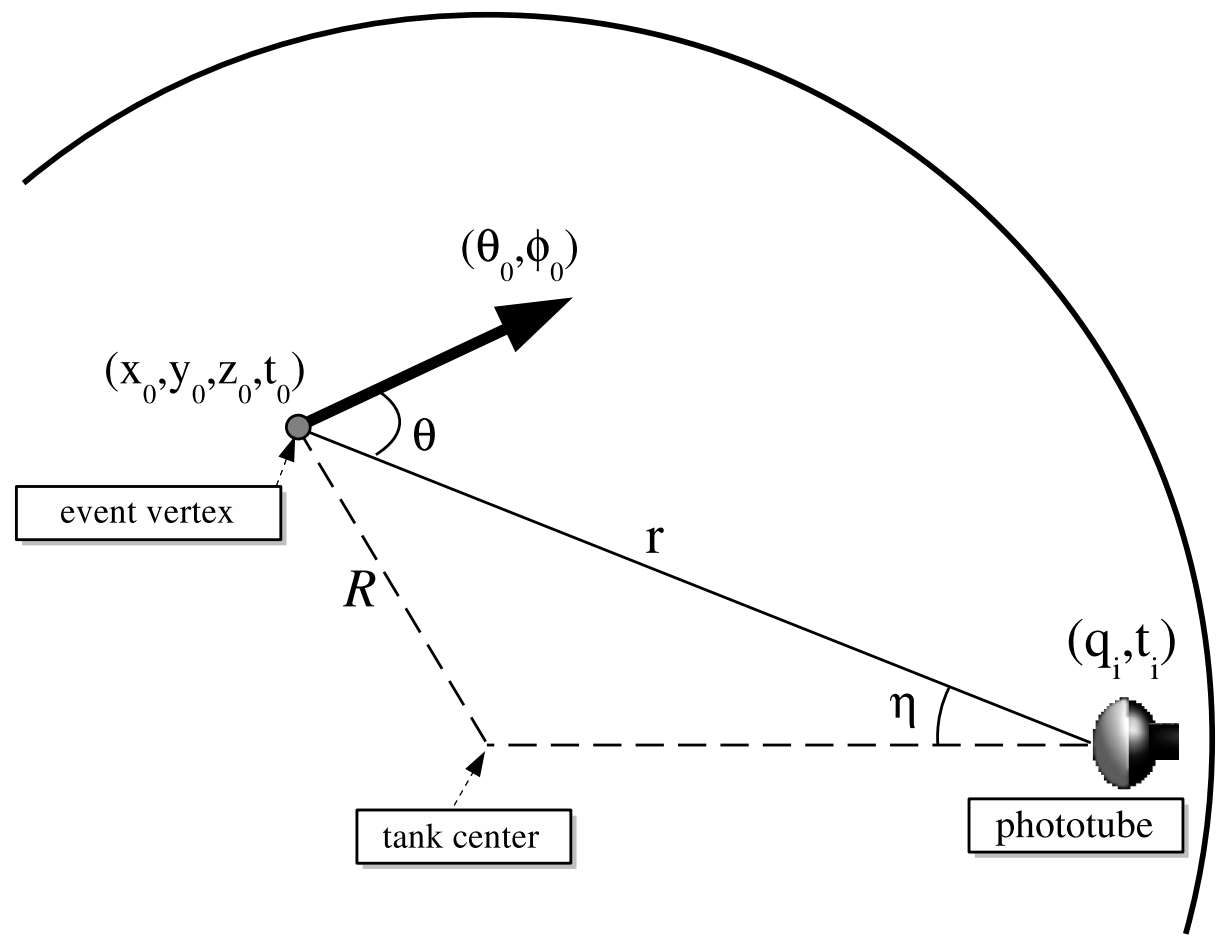

Figure 3.8: Schematic of the reconstructed geometry defining the values that go into $\mathbf{X}$.

- $\epsilon$ is the quantum efficiency of the PMTs. 
- $\phi$ is the number of photons per solid angle for a given event.

- $\lambda$ is the effective attenuation length. For scintillation $\lambda$ is dependent on where in $R$ the event occurred.

- $f(\cos \eta)$ is the angular acceptance of the PMTs, where $\eta$ is the angle of the incident light with respect to the PMT normal.

- $F(E, \cos \theta, R)$ is the angular emission profile with $\theta$ being the angle between the particle's direction and the direction from the vertex to the PMT.

- $\operatorname{Corr}(E, \cos \alpha)$ is a correction function for the probability of the proton leaving the detector before depositing all of its energy. $\alpha$ is the angle between the line from the center of the tank and the event direction.

The best fit $\mathbf{X}$ for a given event with a specific particle hypothesis is when the following likelihood is maximized

$$
\mathcal{L}(\mathbf{X})=\prod_{\text {nohits }} f_{q}(0, \mathbf{X}) \prod_{i, h i t s} f_{q}\left(q_{i}, \mathbf{X}\right) f_{t}\left(t_{i}, \mathbf{X}\right)
$$

In Eq. (3.1) $q_{i}$ and $t_{i}$ are the measured charge and time for the $i$ th PMT, $f_{q}$ is the probability distribution function (PDF) for the $i$ th $\mathrm{PMT}$ to measure $q_{i}$ given $\mathbf{X}$, and $f_{t}$ is the PDF $t_{i}$ will be measured given $\mathbf{X}$. $f_{q}$ and $f_{t}$ can be written in terms of $\mu_{i}$ by

$$
\begin{aligned}
& f_{q}\left(q_{i}, \mathbf{X}\right)=f_{q}\left(q_{i}, \mu_{i}(\mathbf{X})\right) \\
& f_{t}\left(t_{i}, \mathbf{X}\right)=f_{t}\left(t_{i}, \mu_{i}(\mathbf{X}), E\right)
\end{aligned}
$$

Independent of particle hypothesis, the charge likelihood $f_{q}(q>0, \mu)$ was measured by laser calibration data in the MiniBooNE detector, see Ref. [68]. $f_{t}\left(t_{i}, \mu_{i}(\mathbf{X}), E\right)$ is calculated from

$$
f_{t}\left(t_{c o r r}, \mathbf{X}, E\right)=\frac{\mu_{s c i}}{\mu_{s c i}+\mu_{c e r}} f_{i}^{s c i}\left(t_{c o r r}, \mu_{s c i}, E\right)+\frac{\mu_{c e r}}{\mu_{c e r}+\mu_{s c i}} f_{i}^{c e r}\left(t_{c o r r}, \mu_{c e r}, E\right)
$$


where $t_{\text {corr }}$ is the time of the event corrected for the photon propagation time from the vertex of the event to the PMT. Cherenkov light's time PDF is assumed to be Gaussian distribution while scintillation's time PDF is modeled by convolution of a Gaussian with two exponentials. The exponentials represent the time response of the scintillator and the Gaussian represents the response of the PMTs. More detail about the MiniBooNE reconstructed method is given in Ref. [79]. Details about the NCFitter is given in Ref. [82]. Results from using the NCFitter for $\nu$-Mode-NCE cross section analysis is given in Ref. [50] and for $\bar{\nu}$-Mode-NCE cross section in Ref. [51]. 


\section{CHAPTER 4}

\section{DATA SET DISTRIBUTIONS}

Selection criteria were used to enhance the predicted dark matter (DM) neutral-current elastic (NCE) signal in the off-target data set. In addition, three other distributions were included to constrain the flux and neutrino cross section systematic uncertainties on the DM sample.

The four data sets used in this analysis were: $\nu$-Mode-CCQE $\left(\mathrm{CCQE}_{\nu}\right), \nu$-Mode-NCE $\left(\mathrm{NCE}_{\nu}\right)$, off-target-CCQE $\left(\mathrm{CCQE}_{\mathrm{Off}}\right)$ and off-target-NCE $\left(\mathrm{NCE}_{\mathrm{Off}}\right)$. Here $\mathrm{CCQE}_{\nu}, \mathrm{NCE}_{\nu}$, $\mathrm{CCQE}_{\mathrm{Off}}$, and $\mathrm{NCE}_{\mathrm{Off}}$ refer to the selection sample that was used and are defined in Sec. 4.1. $\nu$-Mode data comes from runs when the beam was hitting the beryllium target and the horn was focusing positive charge mesons; off-target data comes from runs where the beam was steered past the target to hit the beam dump and the horn was powered off. The off-target mode was run to reduce the number of decay-in-flight neutrinos being produced since all neutrino interactions are counted as background in a DM analysis. The cuts described are built upon Refs. [69, 83] for charged-current quasielastic (CCQE) and Refs. [50, 82] for NCE.

$\mathrm{CCQE}_{\nu}$ and $\mathrm{CCQE}_{\mathrm{Off}}$ are used to constrain flux systematics, and $\mathrm{NCE}_{\nu}$ constrains cross section systematics for $\mathrm{NCE}_{\mathrm{Off}}$, the signal channel. This chapter will discuss the selection cuts for both CCQE and NCE, how the off-target flux was determined, and the systematic uncertainty on each distribution. The chapter will conclude with a summary of the four 
distributions that were used.

\subsection{EVENT SELECTION CUTS}

Cartoons of NCE and CCQE events in the MiniBooNE detector are shown in Fig. 4.1. The definition of NCE and CCQE events follow from previous MiniBooNE analyses where

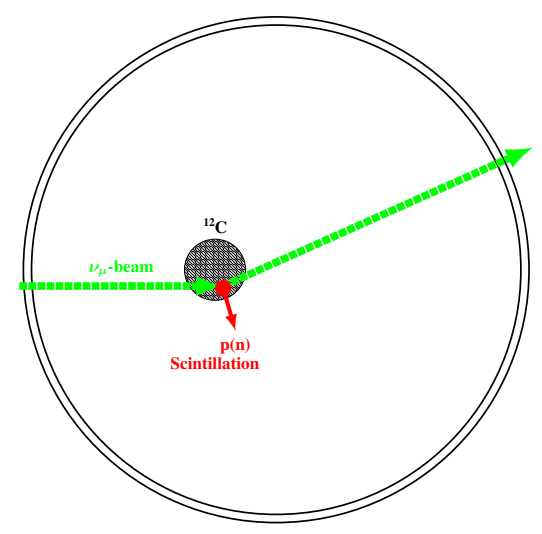

(a) NCE

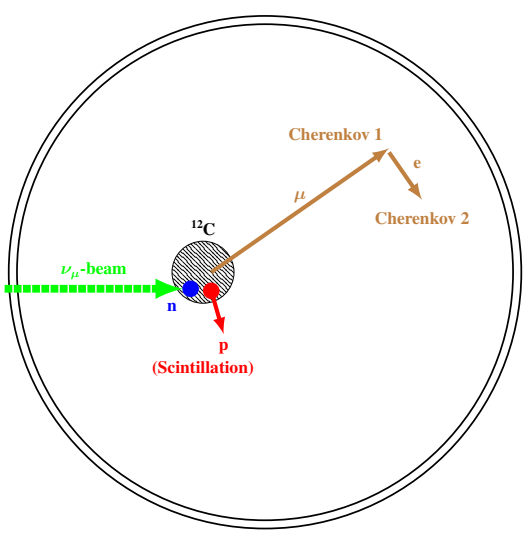

(b) CCQE

Figure 4.1: Cartoon of what a typical (a) NCE and (b) CCQE events contains in the MiniBooNE detector.

a NCE (CCQE) candidate event is really NCE-like (CCQE-like). The "like" is added because the cut is not based on the true neutrino interaction but the final state particles. For example the true neutrino interaction could have been CCQE but the muon did not leave the carbon atom and so only the proton was seen. This event is then seen as NCE-like.

In contrast, the DM signal is assumed to be purely DM scattering off a nucleus, i.e. NCE. For the rest of this discussion the "like" will be dropped. When cuts are being described NCE (CCQE) will refer to NCE-like (CCQE-like), but when the actual processes are being discussed NCE and CCQE refer to the true interaction.

A NCE event is expected to have a single outgoing nucleon, assuming no other final state interactions, while a CCQE event is expected to have an outgoing muon and an outgoing 
proton. All MiniBooNE analyses to-date have been fully contained analyses, therefore, for the CCQE event selection, the muon is required to decay in the MiniBooNE detector and the Michel electron is detected. The lifetime of a muon in mineral oil is about $2 \mu \mathrm{s}$. The CCQE analysis search selecting for a muon followed by an electron is simplified by the high Cherenkov threshold of protons compared to muons in mineral oil. For CCQE events the Cherenkov light produced by the muon will dominate the scintillation light produced from the proton.

\subsubsection{NCE SELECTION CRITERIA}

The NCE cuts in this analysis follow those of Ref. [51] and are shown in Table 4.1. An individual 19.2 $\mu$ s DAQ window with all photomultiplier tube (PMT) hit information is called an event, therefore, groups of hits localized in time inside the DAQ window are called subevents. A hit is defined as a single PMT fired above threshold regardless of the amount of charge on the PMT. A subevent is defined as a group of hits, independent of spatial location, that are not separated by more than $10 \mathrm{~ns}$ and must have at least 10 hits. NCE events are expected to produce a single subevent in the DAQ window resulting in NCE1.

NCE2 ensure the event is coming from the beam. This assumes the interacting particle, neutrino or $\mathrm{DM}$, is traveling near the speed of light, $\beta \simeq 1$. With $491 \mathrm{~m}$ of mainly dirt from the front of the beam dump to the center of the detector to stop any charged or slow moving neutral particles, it is reasonable to believe events outside the beam timing region are coming from only beam unrelated sources.

NCE3 is split between requiring a minimum number of tank hits for reconstruction

purposes and reconstructed nucleon kinetic energy $T_{N}^{\text {reco }}$ region where the neutrino NCE reaction dominates over beam-related background events. In simulations, there were no events that violated the tank hit cut but satisfy the energy cut. This relationship between 


\begin{tabular}{ll}
\hline cut \# & \multicolumn{1}{c}{ description } \\
\hline neutral-current elastic $(\mathrm{NCE})$ & \# total subevents $=1$ \\
NCE1 & event time widnow $4.4<T(\mu \mathrm{s})<6.4$ \\
NCE2 & \# tank hits $>12$ and \\
NCE3 & reconstructed kinetic energy between 0.035 and $0.65 \mathrm{GeV}$ \\
& NCE4 of veto hits $<6$ \\
NCE5 & Reconstructed vertex radius $<500 \mathrm{~cm}$ \\
NCE6 & $p / e$ log-likelihood ratio $<0.42$ \\
NCE7 & Trigger with less than 60 hits $10 \mu \mathrm{s} \mathrm{before} \mathrm{event} \mathrm{trigger}$ \\
& \\
charged-current quasielastic $(\mathrm{CCQE})$ \\
CCQE1 & $\#$ total subevents $=2$ \\
CCQE2 & all subevents, \# of veto hits $<6$ \\
CCQE3 & 1st subevent, \# of tank hits $>200$ \\
CCQE4 & 1st subevent, event time window, $4.4<T(\mu \mathrm{s})<6.4$ \\
CCQE5 & 1st subevent, reconstructed vertex radius $<500 \mathrm{~cm}$ \\
CCQE6 & 1st subevent, kinetic energy $>200 \mathrm{MeV}$ \\
CCQE7 & 1st subevent, $\mu / e$ log-likelihood ratio $>0.0$ \\
CCQE8 & $\mu-e$ vertex distance $>100 \mathrm{~cm}$ and \\
& $\mu-e$ vertex distance $>\left(500 \times T_{\mu}(\mathrm{GeV})-100\right) \mathrm{cm}$ \\
\hline
\end{tabular}

Table 4.1: List of cuts for the NCE and CCQE event selections. 
the tank hits and reconstructed energy was used to make sure there was no overlap between the minimum number of tank hits and $T_{N}^{\text {reco }}$. The upper bound on $T_{N}^{\text {reco }}$ was set where the neutrino background to NCE events became greater than the NCE signal when running in $\nu$-Mode.

To reduce the number of beam-unrelated background (BUB), where the interacting particle was generated outside the detector, NCE4 was used. With the back-to-back nature of the PMT positioning and the noise of the PMTs the maximum number of allowed veto PMTs was set to 6. Cosmic muons and electrons produced outside are greatly rejected by this cut. The veto hit cut reduces the cosmic ray background by a factor of $10^{4}$ [68].

The apex of the tank PMTs are at a radius of $\sim 550 \mathrm{~cm}$. A fiducial volume, the volume required to contain the starting position of the track, was determined for reconstructed efficiency (NCE5). The $\mathrm{NCE}_{\nu}$ cross section analysis applied an energy-dependent fiducial volume cut to reduce more of the dirt background [50]. For this analysis an energy independent fiducial volume was used for all distributions.

NCE6 separates events that are more Cherenkov- or scintillation-like by utilizing the difference in the time response of the PMTs between Cherenkov and scintillation light. All events are fitted with both hypothesis and NCE6 is a ratio of the two hypothesis, denoted as $L_{t}^{p / e}$, to reject more Cherenkov like events. Details of a study to search for a $T_{N}^{\text {reco }}$ dependent $L_{t}^{p / e}$ cut is given in appendix A. The gain from the $T_{N}^{\mathrm{reco}}$-dependent cut was not enough to merit the additional complication. The cut $L_{t}^{p / e}<0.42$ determined by Refs. [50, 70], was used.

NCE7 uses previous trigger information (see Sec. 3.2.1) to further reduce the amount of BUB. For an event to survive the cut the event cannot be correlated with a trigger that contained more than 60 tank hits within $10 \mu \mathrm{s}$ of the events trigger. The following explains the study done to set this cut. 


\section{PREVIOUS TRIGGER CUT STUDY}

A study was done to see if adding a cut using the previous trigger information (see Sec. 3.2.1) would reduce the amount of BUB in the $\mathrm{NCE}_{\text {Off }}$ sample. All combinations of the DET, VETO triggers and time difference between previous trigger and the subevent trigger $(\Delta T)$ were considered. To find the best combination of DET, VETO, and $\Delta T$ the following equation for the figure of merit $\mathcal{F}$ was maximized

$$
\mathcal{F}=\frac{N_{\text {Beam }}-N_{\text {BUB }}}{\sqrt{N_{\text {Beam }}+\sigma_{N_{\text {BUB }}}^{2}}},
$$

where $N_{\text {Beam }}\left(N_{\text {BUB }}\right)$ is the number of beam (beam-unrelated) events after applying a DET, VETO, and $\Delta T$ cut, $\sigma_{N_{\text {BUB }}}^{2}$ is the uncertainty on $N_{\text {BUB }} . N_{\text {BUB }}$ is measured by using the STROBE trigger (see Sec. 3.2.2). $\mathcal{F}$ is approximately equal to one over the fractional uncertainty on the beam-related background (BRB).

The simulations do not simulate the previous trigger, therefore $N_{\text {Beam }}$ has to come from beam data. To keep the DM search blind, this study only used the first $0.32 \times 10^{20}$ POT of the off-target data, $\sim 17 \%$ of the total sample. Two different trigger types are defined, Word and Cluster. A single previous trigger's clock-tick information is called a Word. A Cluster is defined as a group of Words with no more than two clock-ticks separating two continuous Words, as shown in Fig. 4.2. The trigger information in a Cluster is the logical

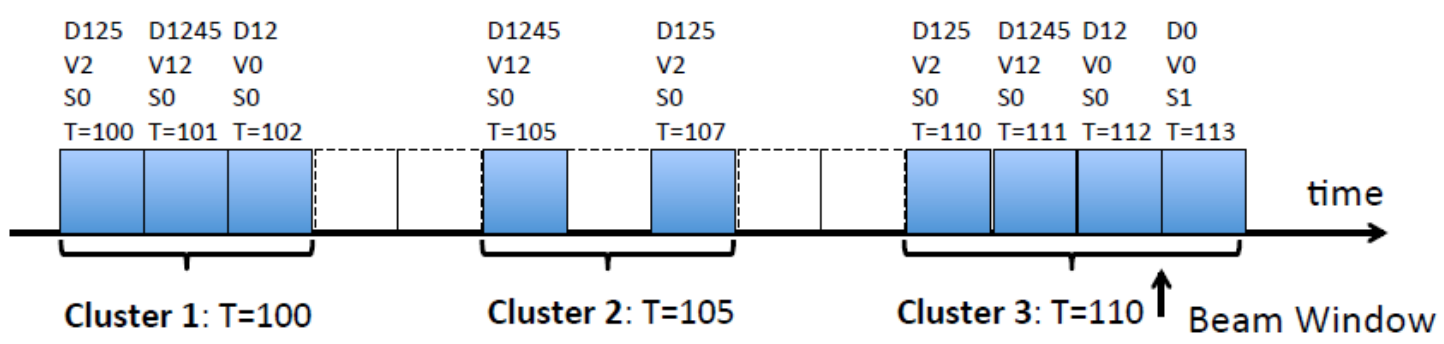

Figure 4.2: Diagram showing how previous trigger Clusters are made.

OR of all the Words that make up the cluster. As shown in Fig. 4.2, if an internal event occurs a clock-tick or two before an external trigger the Cluster would register the internal 
trigger as part of the external trigger. A detailed analysis showed cutting on Word had a higher efficiency than cutting on Clusters [84]. The following description focuses on the Words analysis .

To require a previous trigger to be correlated with the subevent's trigger, the previous trigger cannot come from an external trigger. Figure 4.3 shows an example $\Delta T$ distribution for both $N_{\text {Beam }}$ and $N_{\text {BUB }}$ applying all the other NCE selection cuts (NCE0) and taking the opposite of the time likelihood cut (NCE1).

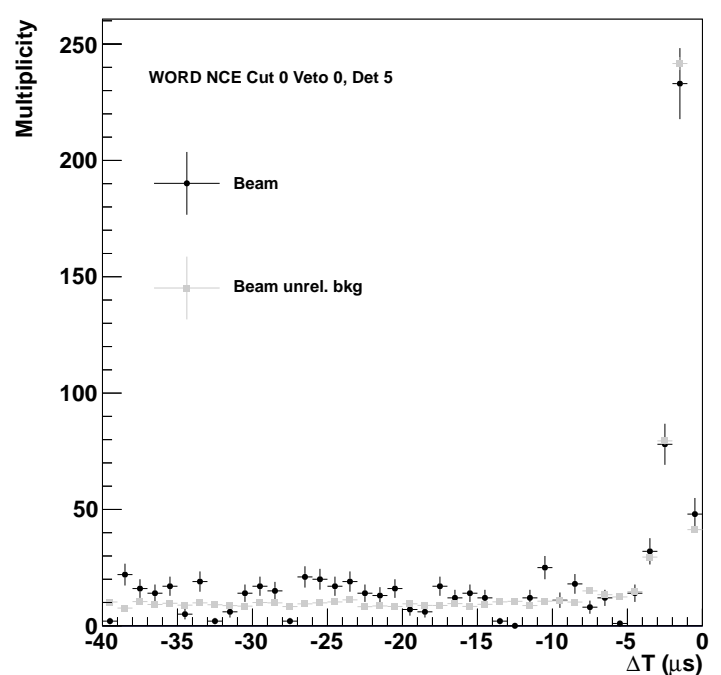

(a) Normal $L_{t}^{p / e}$ cut

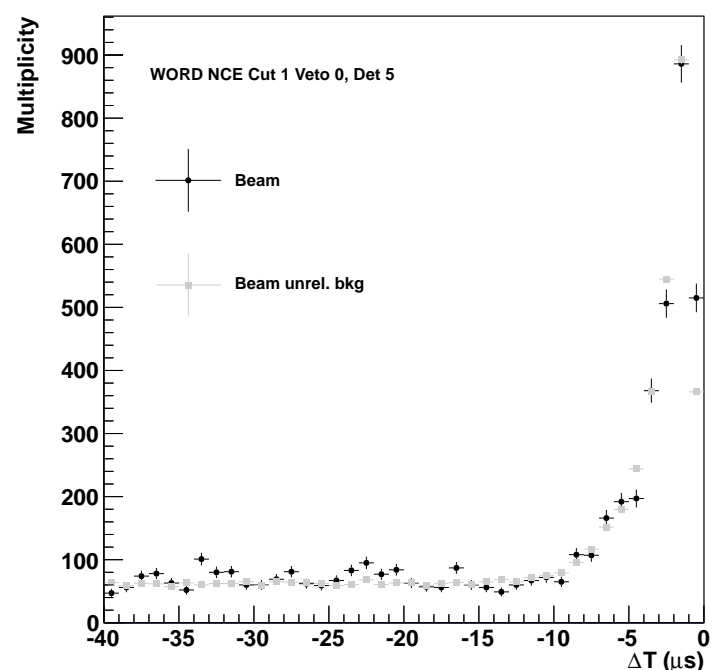

(b) Reverse $L_{t}^{p / e}$ cut

Figure 4.3: Example time difference between current event and previous triggers distributions for beam and beam-unrelated data where the previous trigger has the DET5 bit set. (a) Uses the normal time likelihood cut while (b) takes the inverse.

The lifetime of the NCE0 distribution is $0.8 \mu \mathrm{s}$, which is not consistent with the muon lifetime. NCE1, on the other hand, has a lifetime of $2.2 \mu \mathrm{s}$, which is consistent with the muon lifetime. The fact NCE0 does not produce a muon lifetime suggests that events in the detector with a previously correlated trigger are from BUB neutrons most likely produced by muon spallation in the rock where the muon missed the MiniBooNE detector. 
To calculate the uncorrelated, or accidental rate the following was used

$$
\mathcal{R}=\frac{\# \text { events fail cut in time window }}{(\# \text { original events) (length of time window) }}
$$

for a period in $\Delta T$ where no correlated signal is expected. For this analysis the window between -20 and $-40 \mu$ s was used. The accidental efficiency is calculated by

$$
\mathcal{P}_{S}=1-\mathcal{R} \Delta T
$$

Fig. 4.4 shows the percent change in $\mathcal{F}$ before and after applying a previous trigger cut for all the different combinations of DET, VETO, and $\Delta T$. The combination that gives the maximum $\mathcal{F}$ is DET5 with no VETO constraint at $\Delta T=14 \mu$ s. With little change in $\mathcal{F}$ and lower $\mathcal{R}$, the cut chosen was to reject events with a previous trigger containing at least DET5 with no external trigger within $10 \mu$ s of the subevent's trigger. The BUB is reduced by $42.5 \%$ while only reducing the number of beam related events by $(4.7 \pm 0.2) \%$. It was assumed the previous trigger rate for off-target was the same for $\nu$-Mode.

\subsubsection{CCQE SELECTION EVENTS}

The CCQE cuts in this analysis follow those of Ref. [83] and are shown in Table 4.1. With the expectation of detecting the muon followed later in time by the decay electron, the number of subevents required equals two. The veto rejection cut is required on all subevents while the event time window cut, tank hit cut, and fiducial volume are all requirements only on the first subevent. The tank hit cut was set to 200 to distinguish between electrons and muons at low visible energy. The log-likelihood cut to separate muons from electrons uses the full likelihood, not just the time likelihood used in the NCE selection. A distance cut between the starting positions of the first and second subevent reduces events where the first and second subevent are uncorrelated.

Refs. $[69,83]$ discuss a side band analysis where the number of allowed subevents was set to three and the distance cut between the first and second subevent was removed. This was 


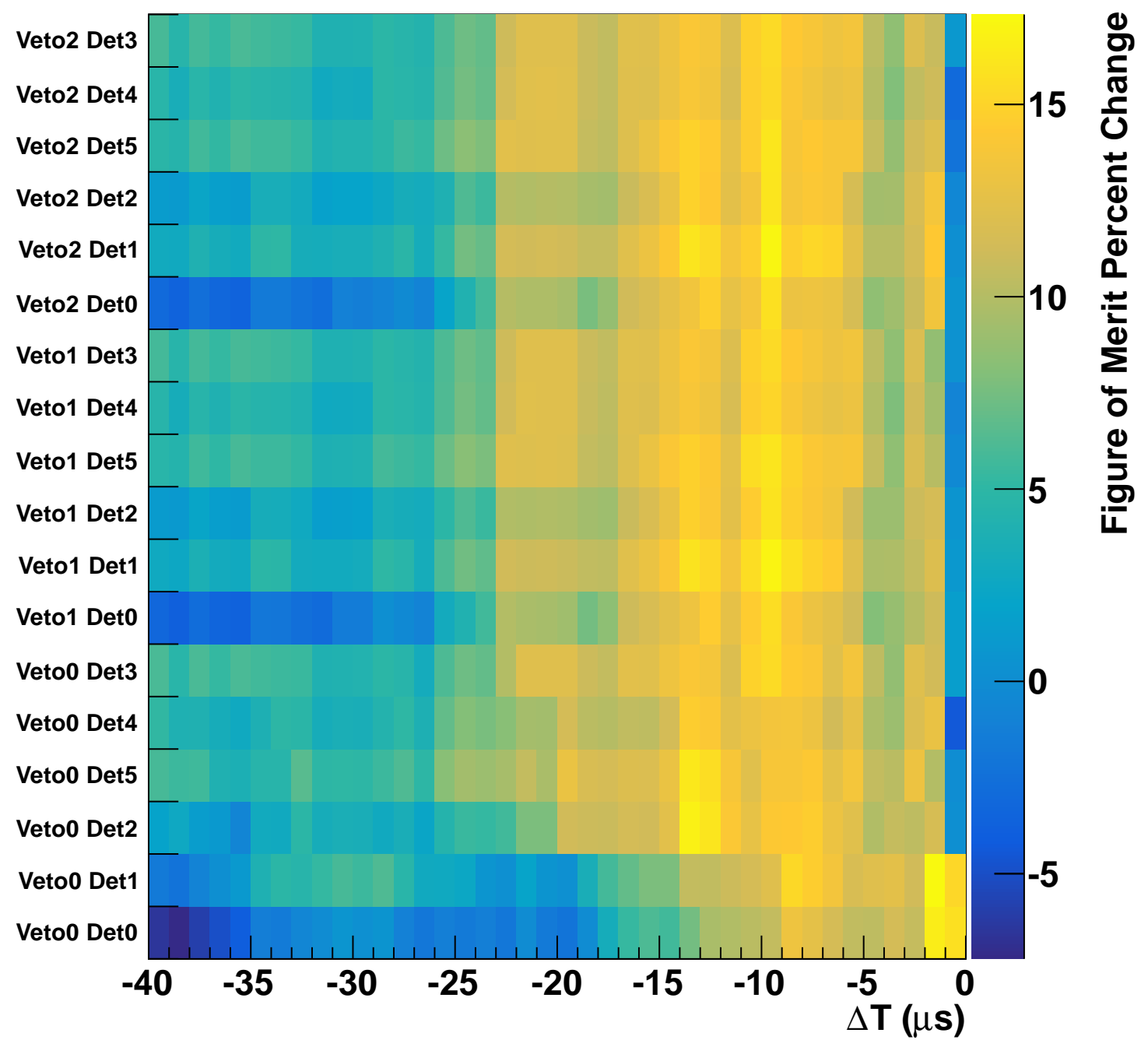

Figure 4.4: Percent change of $\mathcal{F}$ after applying a pre-trigger cut as a function of DET, VETO, and $\triangle T$. A DET0 or VETO0 means there was no constraint on that type of trigger, i.e. DET0 and VETO0 means any trigger not caused by an external trigger. 
to measure the $\mathrm{CC} 1 \pi^{+}$contribution to the CCQE-like background. With limited statistics in $\mathrm{CCQE}_{\mathrm{Off}}$, the correction to the low energy $\mathrm{CC} 1 \pi^{+}$cross section used in $\mathrm{CCQE}_{\nu}$ was used for $\mathrm{CCQE}_{\mathrm{Off}}$.

\subsubsection{DEFINITION OF $Q_{Q E}^{2}$}

Kinematic equations relate reconstructed energy/angle to the four-momentum transfer $Q^{2}$. Both CCQE and NCE label the reconstructed four-momentum under the quasi-elastic hypothesis with $Q_{Q E}^{2}$. For CCQE, $Q_{Q E}^{2}$ is defined by [69]

$$
Q_{Q E}^{2}=-m_{\mu}^{2}+2 E_{\nu}^{Q E}\left(E_{\mu}-\sqrt{E_{\mu}^{2}-m_{\mu}^{2}} \cos \theta_{\mu}\right)
$$

where

$$
E_{\nu}^{Q E}=\frac{2 m_{n}^{\prime} E_{\mu}-\left[\left(m_{n}^{\prime}\right)^{2}+m_{\mu}^{2}-m_{p}^{2}\right]}{2\left[m_{n}^{\prime}-E_{\mu}+\sqrt{E_{\mu}^{2}-m_{\mu}^{2}} \cos \theta_{\mu}\right]},
$$

$m_{n}^{\prime}=m_{n}-E_{b}$ is the neutron mass adjusted for the binding energy of carbon, set to $34 \mathrm{MeV}$, $m_{p}$ is the mass of the proton, $m_{\mu}$ is the mass of the muon, $E_{\mu}$ is the total reconstructed energy under the muon hypothesis, and $\theta_{\mu}$ is the reconstructed angle with respect to the beam direction. For NCE, $Q_{Q E}^{2}$ is defined as [50]

$$
Q_{Q E}^{2}=2 m_{N} T_{N}^{\mathrm{reco}}
$$

where $m_{N}$ is the mass of the nucleon. This analysis uses the definitions of $Q_{Q E}^{2}$ instead of the true $Q^{2}$, because the true $Q^{2}$ is impossible to define experimentally, and $Q_{Q E}^{2}$ is a well-defined approximation of $Q^{2}$ based on reconstructed quantities. In later sections $\mathrm{CCQE}_{\nu}, \mathrm{NCE}_{\nu}, \mathrm{CCQE}_{\mathrm{Off}}$, and $\mathrm{NCE}_{\mathrm{Off}}$ will be plotted as functions of $Q_{Q E}^{2}$.

\subsection{OFF-TARGET NEUTRINO SIMULATION}

With approximately $500 \mathrm{~m}$ of distance and shielding between the beam dump and the MiniBooNE detector the BRB is assumed to be composed of only neutrino induced events. 
$\mathrm{BRB}$ are arranged into two subcategories, 1) beam related events interacting in the detector $\left(\nu_{\text {det }}\right)$, and 2) beam related events that interact in the dirt around the detector $\left(\nu_{\text {dirt }}\right)$. To understand these backgrounds, the neutrino flux needs to be well understood. For the off-target neutrino flux, updates were made to BooneG4BEAm to better simulate the offtarget running conditions. These updates include the beam profile and the addition of materials that change the on-target flux by less than a percent. This section will discuss theses changes, and show the official off-target flux prediction.

\subsubsection{SIMULATED GEOMETRY}

Materials listed in Table 4.2 were added to BooNEG4BEAm. Most of the geometry in the simulations are either a rectangular box or are concentric cylinders with an inner and outer radius as shown in Fig. 4.5. The inner radius is bound between 0 and the length of the outer radius. An inner radius equal to 0 means there is no gap in the material at the center of the cylinder. The added materials came in the following classes: 1) material that should have been included due to having no inner radius, 2) material with an inner radius that has the off-target beam now interacting with the material, and 3) material that is down stream of interacting material but does not interact with the beam. The last class is added because although the material does not interact with the beam directly, any particles produced upstream can see the material thus generating more secondary particles.

To simulate the effect of particles scattering off wires, multiwire planes were added to the simulations. MW876 and MW875 were positioned an arbitrary distance in-front of the start of the beam. An end cap was discovered during the beam pipe inspection that was not in the simulations. Without having much information about the material or thickness of the end cap, a steel end cap located $49.92 \mathrm{~m}$ from the target center with a half length $0.516 \mathrm{~cm}$ was added to the simulation. With this position, an air gap of $9.91 \mathrm{~cm}$ between the end cap and the steel beam dump was assumed. Schematics of the entire beam-line 


\begin{tabular}{|c|c|c|c|c|}
\hline Geometry & Material & $\begin{array}{c}\text { Inner Radius } \\
\text { (mil) }\end{array}$ & $\begin{array}{c}\text { Half Length } \\
\text { (mil) }\end{array}$ & $\begin{array}{l}\text { Location } \\
\qquad(\mathrm{cm})\end{array}$ \\
\hline \multirow{3}{*}{ Vacuum } & \multirow{3}{*}{ vacuum } & 0 & 51929 & -214.1 \\
\hline & & 0 & 19685 & -395.1 \\
\hline & & 0 & 7874 & -464.1 \\
\hline \multirow{2}{*}{ MW wires } & \multirow{2}{*}{ W } & 0 & 1.5 & -476.1 \\
\hline & & 0 & 1.5 & -475.6 \\
\hline \multirow{2}{*}{ MWTGT foils } & \multirow{2}{*}{$\mathrm{Al}$} & 250 & 5 & -103.4 \\
\hline & & 250 & 5 & -98.3 \\
\hline MWTGT Ceramic & $\mathrm{Al}_{2} \mathrm{O}_{3}$ & 625 & 40 & -100.7 \\
\hline \multirow{2}{*}{ MWTGT wires } & \multirow{2}{*}{ W } & 0 & 1.5 & -100.8 \\
\hline & & 0 & 1.5 & -100.6 \\
\hline End of vacuum window & $\mathrm{Ti}$ & 0 & 0.6 & -82.2 \\
\hline Target Back Plate & $\mathrm{Al}$ & 500 & 250 & -56.2 \\
\hline Target Window & $\mathrm{Be}$ & 0 & 15 & -47.7 \\
\hline Target Base Block & $\mathrm{Al}$ & 543 & 1000 & -45.1 \\
\hline Target BCA & $\mathrm{Al}$ & 575 & 905.5 & -40.3 \\
\hline Upstream fin locator & $\mathrm{Be}$ & 475 & 2500 & -47.6 \\
\hline Downstream fin locator & $\mathrm{Be}$ & 475 & 405.5 & 34.5 \\
\hline \multirow{2}{*}{ Horn Window } & \multirow{2}{*}{$\mathrm{Al}$} & 0 & 5 & 143.9 \\
\hline & & 13012 & 375 & 143.9 \\
\hline
\end{tabular}

Table 4.2: List of geometries upstream or around the target needed for off-target simulations. The location is with respect to the center of the target. When there is multiple information given for a specific geometry that is either because there are multiple pieces not connected, e.g. MWTGT foils, or the geometry spans over several mother volumes, e.g. Vacuum. BCA stands for bellows contact assembly. 


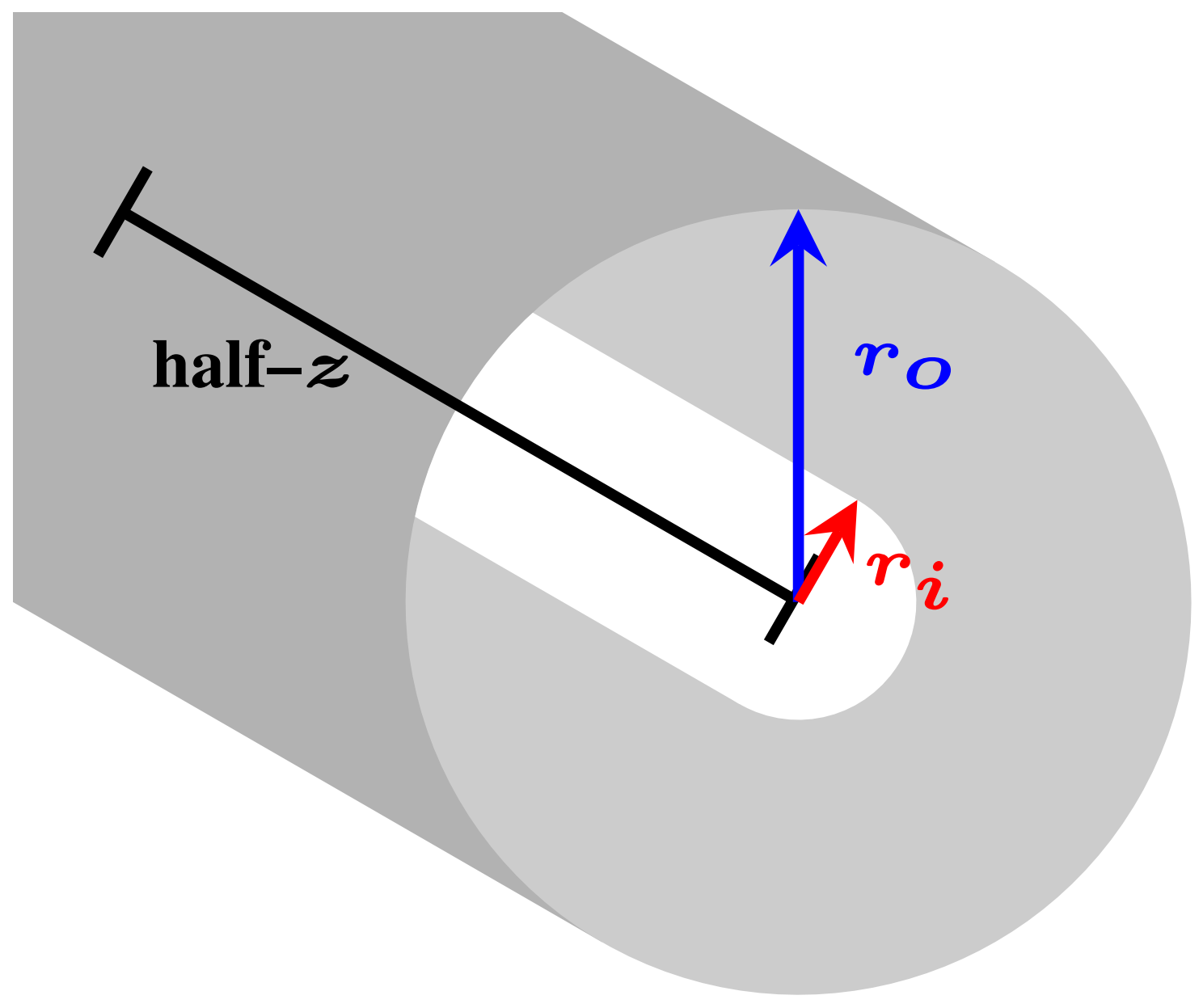

Figure 4.5: Schematic of a tube shape material in the simulations. The dark outer cylinder is the material of radius $r_{o}$ and the inner white cylinder is hollow with radius $r_{i}$. The half length of the tube (half- $z$ ) is also shown. 
simulation can be found in Figs. 4.6a to 4.6d.

BMLN CAVE DKO1 IABC DKTN FABC
Dolomite

(a) The entire BooNE Beam-line simulated geometry. BMLN stands for beam-line, CAVE is where the target hall sits, DK01 contains the upstream decay pipe, IABC houses the $25 \mathrm{~m}$ absorber, DKTN contains the downstream decay pipe, and FABC houses the $50 \mathrm{~m}$ beam dump.

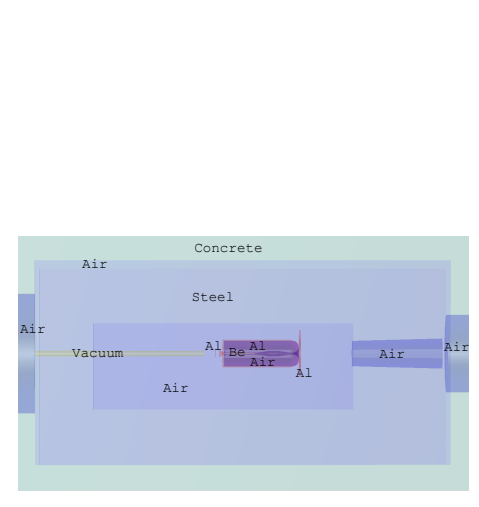

(b) The target hall

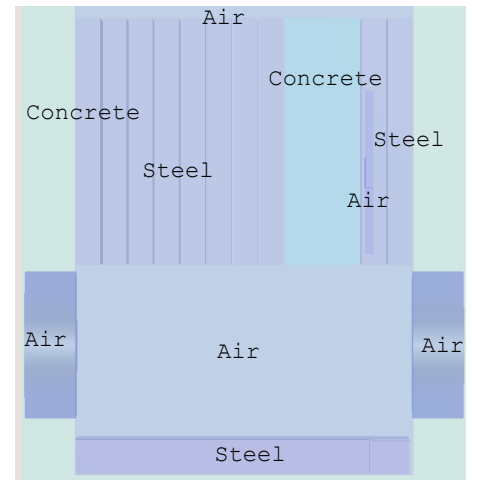

(c) The $25 \mathrm{~m}$ absorber

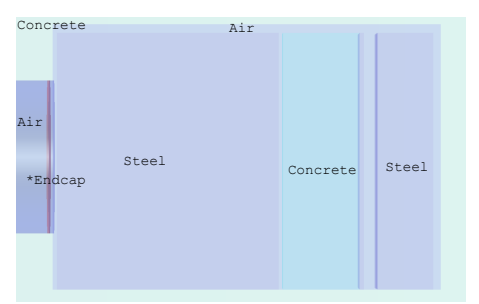

(d) The $50 \mathrm{~m}$ absorber

Figure 4.6: Simulated beamline geometry. (b), (c), and (d) are zoomed in parts shown in (a). The zoomed in parts are not zoomed to the same scale.

\subsubsection{DETERMINING CENTRAL-VALUE FLUX}

The beam starting parameters, Table 4.3 , for the off-target simulation of the neutrino flux were taken from measurements by MW875 and MW876 during off-target running. The beam divergence was determined for on-target running and was not changed for this analysis. Fig. 4.7 gives the $3-\sigma$ profile of the off-target beam at various points along the beamline with different variations in the beam profile.

The nominal beam profile with the updated geometry produced $62 \%$ fewer CCQE events than measured, as shown in Fig. 4.8. Four variations, or excursions, were found to reproduce the number of CCQE events to within $15 \%$ with little scraping of the target. The four 


\begin{tabular}{crcll}
\hline Parameter & Value & Uncertainty & Unit & Extra Description \\
\hline$x$ & -4.098 & 0.44 & $\mathrm{~mm}$ & \\
$y$ & 6.399 & 0.511 & $\mathrm{~mm}$ & \\
$z$ & -4.372 & & $\mathrm{~m}$ & \\
$\theta_{x}$ & 0.93 & 0.21 & $\mathrm{mrad}$ & \\
$\theta_{y}$ & 0.42 & 0.31 & $\mathrm{mrad}$ & \\
$\sigma_{x}$ & 2.88 & 0.19 & $\mathrm{~mm}$ & Beam Spread \\
$\sigma_{y}$ & 1.11 & 0.05 & $\mathrm{~mm}$ & \\
$\sigma_{\theta_{x}}$ & 0.66 & & $\mathrm{mrad}$ & Beam Divergence \\
$\sigma_{\theta_{y}}$ & 0.40 & & $\mathrm{mrad}$ & \\
\hline
\end{tabular}

Table 4.3: The nominal beam off-target staring parameters, where the uncertainties given are RMS errors for the given parameter.
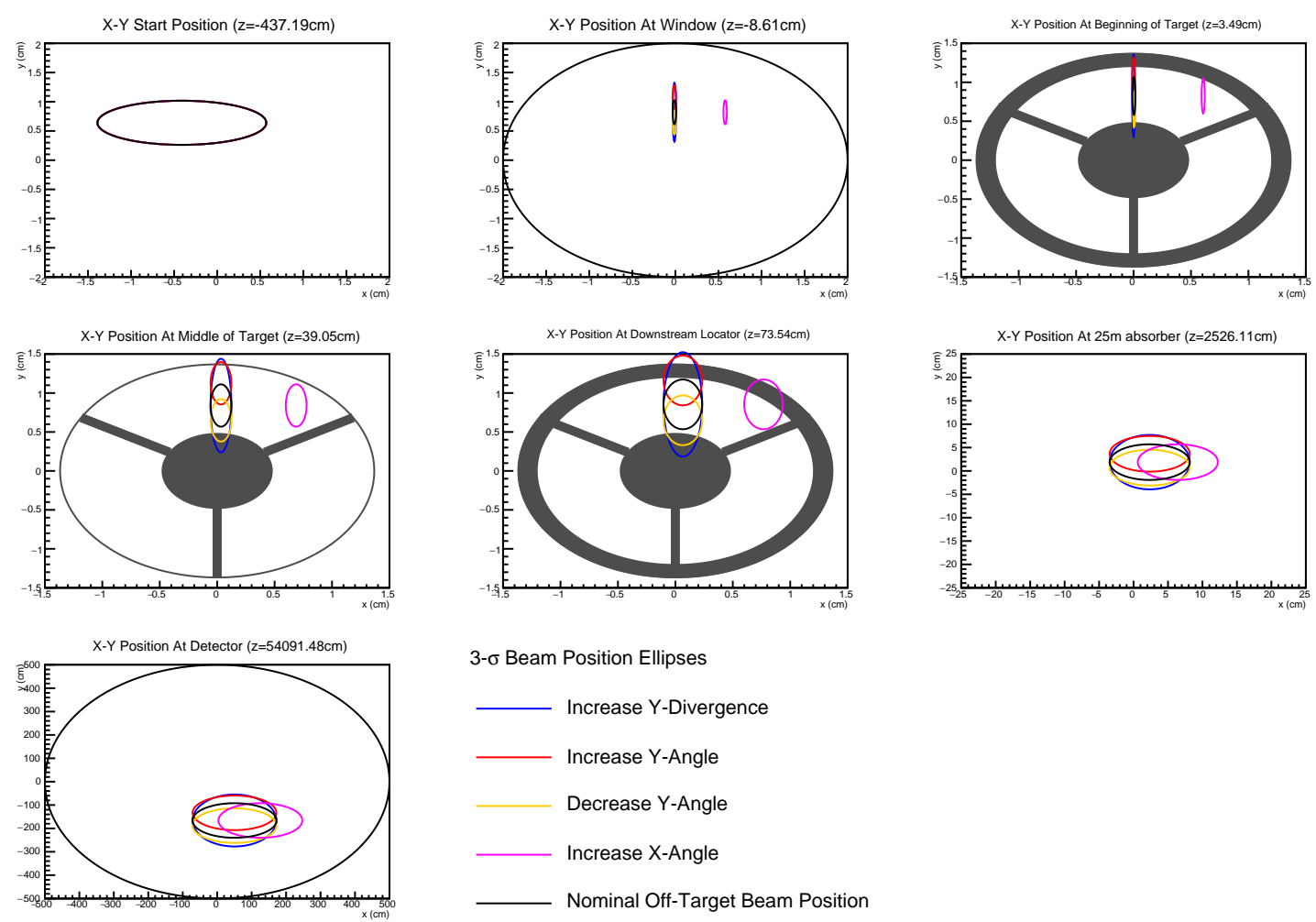

Figure 4.7: 3- $\sigma$ Projections of the beam with various starting parameters given at various points along the beamline. 


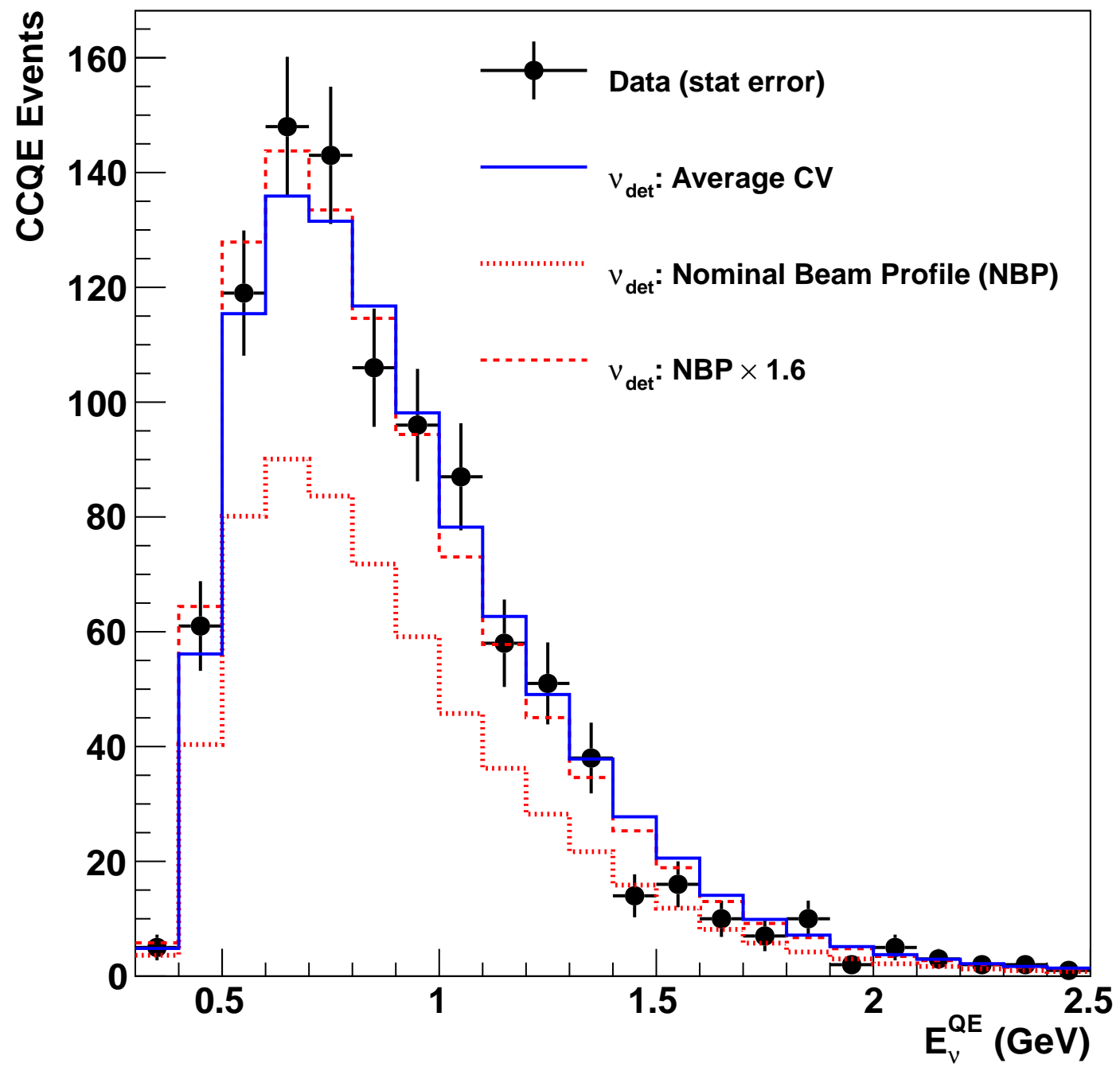

Figure 4.8: Comparing $\mathrm{CCQE}_{\mathrm{Off}} E_{\nu}^{\mathrm{QE}}$ data to three different Monte Carlo Predictions. Dotted red is the output of the nominal off-target beam profile, dashed red is the dotted red scaled by 1.62 , solid blue is the final central-value flux determined by averaging excursions. 
excursions were: 1) decrease $\theta_{y}$ by $\left.0.22 \mathrm{mrad}, 2\right)$ increase $\theta_{y}$ by $\left.0.60 \mathrm{mrad}, 3\right)$ increase $\theta_{x}$ by $1.375 \mathrm{mrad}$, and 4 ) increase $\sigma_{\theta_{y}}$ by $0.02 \mathrm{mrad}$. Each excursion except for the increase of $\theta_{x}$ is within 2- $\sigma$ of the uncertainty from MW875 and MW876 measurements. The central value of the off-target flux $\Phi_{\text {off }}$ was taken to be the average of the four excursions not knowing which one, or combination of, is reality. Fig. 4.9 shows $\Phi_{\text {off }}$ as a function of true neutrino energy $E_{\nu}$ as well as the energy dependent ratio of off-target to $\nu$-Mode flux. $5 \times 10^{10}$ protons on target $(\mathrm{POT})$ were simulated for each excursion.

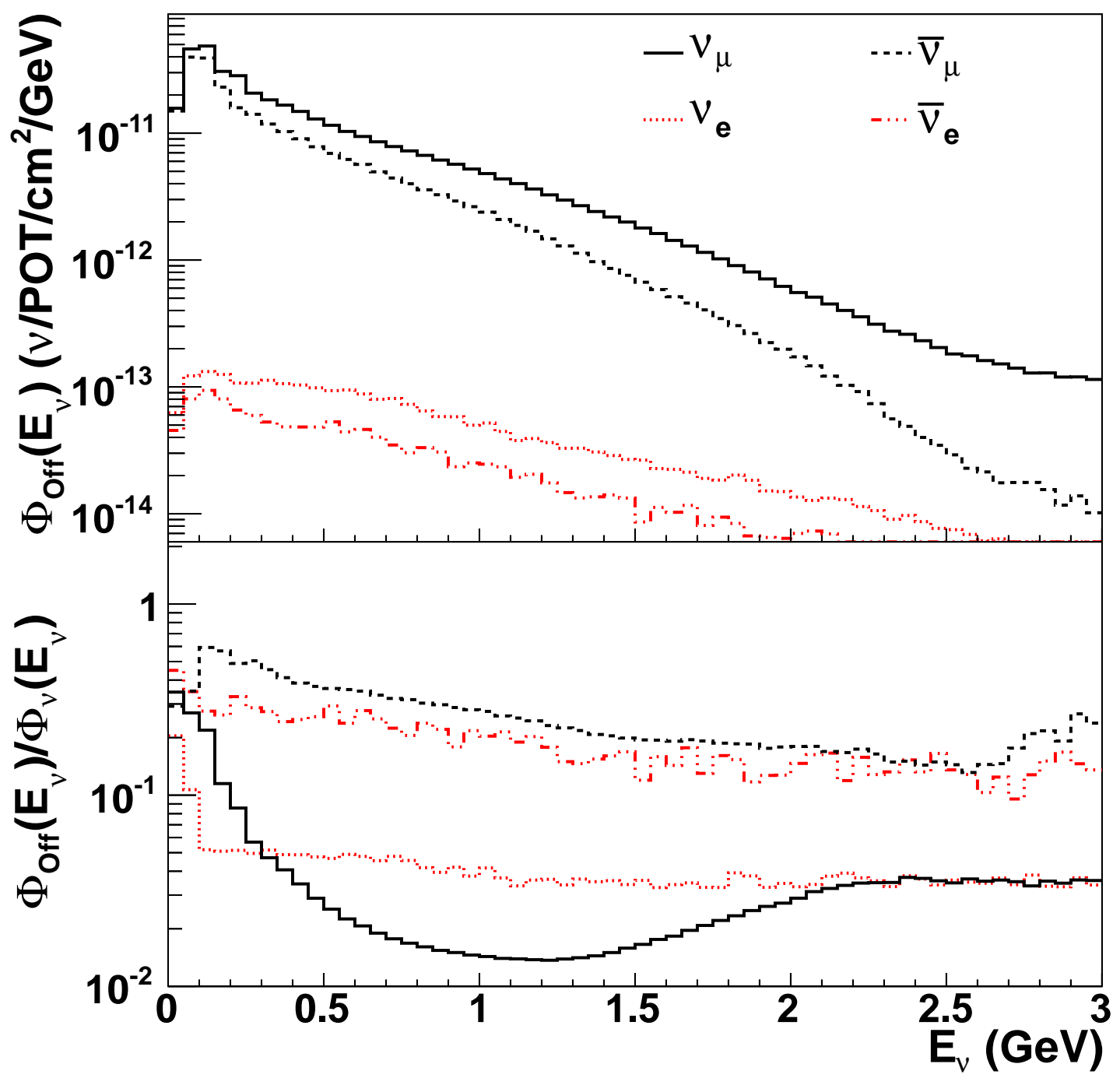

Figure 4.9: (top) The $E_{\nu}$ distribution of the flux in off-target mode. (bottom) The ratio of off-target to $\nu$-Mode flux. Both show each neutrino species. 
Uncertainty in the flux was determined by the nominal beam profile, 1- $\sigma$ excursions around the nominal beam profile, and the excursions that made up the central value. The 1- $\sigma$ excursions and the nominal beam profile were scaled by 1.62 so the nominal beam profile reproduced the number of CCQE events. The energy dependent flux covariance matrix is calculated using

$$
M_{i j}=\sum_{l} \sum_{k}\left(N_{i}^{l, k}-N_{i}^{l, \mathrm{CV}}\right)\left(N_{j}^{l, k}-N_{j}^{l, \mathrm{CV}}\right)
$$

where $i$ and $j$ index the energy bins, $k$ indexes the systematic excursions and $l$ indexes the neutrino species. Fig. 4.10 shows the uncertainty of the total flux with its correlation matrix. The off-target flux is $(1.9 \pm 1.1) \times 10^{-11} \nu \mathrm{POT}^{-1} \mathrm{~cm}^{-2}$ integrated over $0.2<E_{\nu}<3 \mathrm{GeV}$
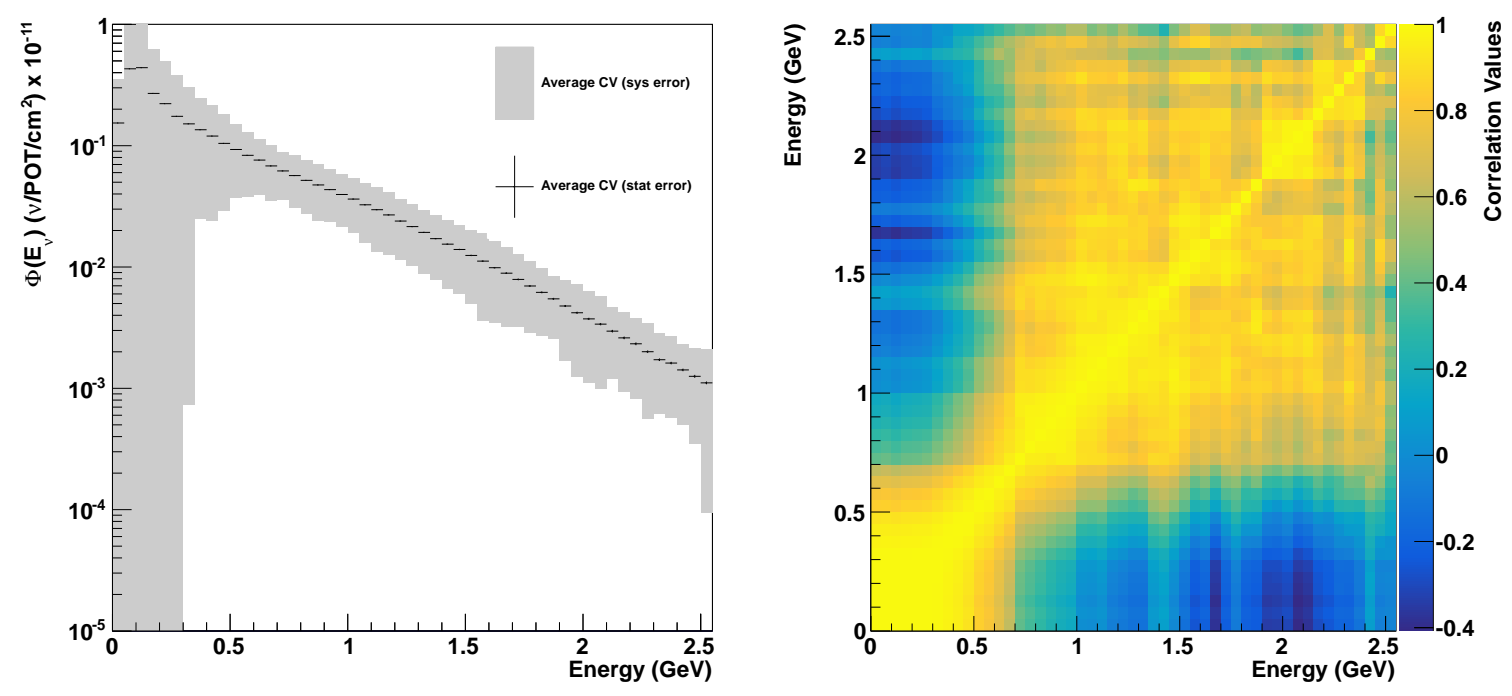

Figure 4.10: (a) The total off-target central-value flux distribution with stat and beam profile systematic errors. (b) The total beam profile systematic correlation matrix.

with a mean energy of $657 \mathrm{MeV}$. The off-target flux has a reduction factor of 26.6 compared to $\nu$-Mode and is softer by $176 \mathrm{MeV}$. The breakdown of the integrated off-target flux by species is given in Table 4.4 .

Fig. 4.11 and Table 4.5 give the breakdown of the parent mesons that produced neutrinos as a function of neutrino species. These are to be compared to Figs. 29 and 30 and Table 

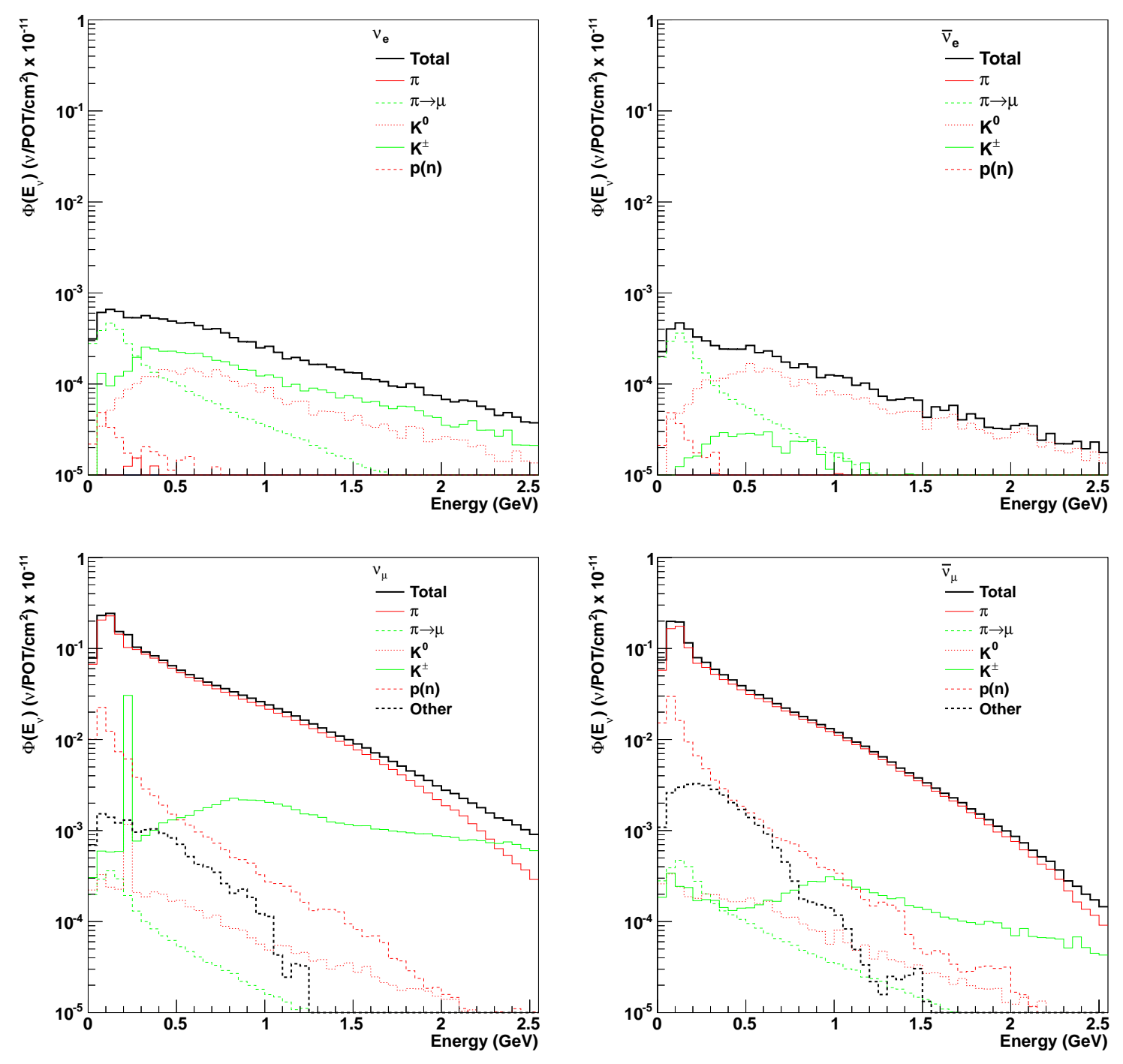

Figure 4.11: Predicted $\nu_{e}$ (top right), $\bar{\nu}_{e}$ (top left), $\nu_{\mu}$ (bottom left), and $\bar{\nu}_{\mu}$ (bottom right) flux at the MiniBooNE detector by secondary particle in beam off-target mode. The black line is the total predicted flux. The rest of the lines (except the dashed black line) are from primary parent meson production. The dashed black line includes all other contributions. 


\begin{tabular}{|c|c|c|c|}
\hline \multirow{2}{*}{$\nu$ species } & \multicolumn{2}{|c|}{$\Phi\left(E_{\nu}\right)\left(\nu \mathrm{POT}^{-1} \mathrm{~cm}^{-2}\right) \times 10^{-12}$} & \multirow{2}{*}{ Perc. Of Tota } \\
\hline & Value & Uncertainty & \\
\hline Total & 19 & 11 & \\
\hline$\nu_{\mu}$ & 12 & 6 & 63.7 \\
\hline $\bar{\nu}_{\mu}$ & 6.6 & 4.7 & 35.4 \\
\hline$\nu_{e}$ & 0.11 & 0.09 & 0.6 \\
\hline $\bar{\nu}_{e}$ & 0.05 & 0.04 & 0.3 \\
\hline
\end{tabular}

Table 4.4: Beam off-target profile systematic percent error independent of energy for the various $\nu$ types, including correlations. $\Phi\left(E_{\nu}\right)$ integrated over $0.2<E_{\nu}<3 \mathrm{GeV}$

9 in Ref. [62]. In particular, the $\nu$-Mode flux is made up of $93.6 \% \nu_{\mu}, 5.9 \% \bar{\nu}_{\mu}$, and $0.5 \%$ $\nu_{e}, \bar{\nu}_{e}$ while the off-target flux is composed of $63.7 \% \nu_{\mu}, 35.4 \% \bar{\nu}_{\mu}$, and $0.9 \% \nu_{e}, \bar{\nu}_{e}$. In on-target running the parent mesons that produce neutrinos that intersect the MiniBooNE detector, are primarily produced in beryllium and aluminium, but in off-target running the break-down is $55 \%$ air, $30 \%$ beryllium, $10 \%$ steel, $3 \%$ aluminium, and $2 \%$ concrete. Above neutrino energies of $500 \mathrm{MeV}$, air and beryllium contribute about equally to the flux.

Table 4.6 shows the breakdown of predicted $\nu_{\text {det }}$ events by true neutrino interaction given by NUANCE. The true neutrino interaction is determined at the primary vertex, i.e. not including any final state interactions. Table 4.6 shows the breakdown for both $\nu$-Mode (top) and off-target (bottom), what is produced by NUANCE before cuts are applied (first column), and after applying NCE (second column)and CCQE (third column) cuts. There is a slight change in the breakdown coming from NUANCE between $\nu$-Mode and off-target. The difference is explained by the softer flux.

Fig. 4.12 shows how the NCE (CCQE) $E_{\nu}^{\text {true }}$ distributions change when comparing true events coming from NUANCE, after applying NCE (CCQE) cuts, and changing from $\nu$-Mode to off-target. The softer off-target neutrino flux causes the peaks in the no-cut samples to be softer when compared to $\nu$-Mode. The $\mathrm{NCE}_{\mathrm{Off}} E_{\nu}^{\text {true }}$ distribution is softer 


\begin{tabular}{|c|c|c|c|c|}
\hline & \multicolumn{2}{|r|}{$\nu_{\mu}$} & \multicolumn{2}{|r|}{$\bar{\nu}_{\mu}$} \\
\hline Flux $\left(\nu \mathrm{POT}^{-1} \mathrm{~cm}^{-2}\right)$ & & $1.91 \times 10^{-11}$ & & $1.25 \times 10^{-11}$ \\
\hline Frac. of Total & & $60.07 \%$ & & $39.31 \%$ \\
\hline \multirow[t]{7}{*}{ Composition } & $\pi^{ \pm}:$ & $89.07 \%$ & $\pi^{ \pm}$ & $87.96 \%$ \\
\hline & $K^{ \pm}:$ & $5.56 \%$ & $p(n):$ & $8.21 \%$ \\
\hline & $p(n):$ & $4.22 \%$ & Other: & $2.50 \%$ \\
\hline & Other: & $0.76 \%$ & $K^{ \pm}:$ & $0.72 \%$ \\
\hline & $K^{0}:$ & $0.26 \%$ & $K^{0}:$ & $0.33 \%$ \\
\hline & $\pi^{ \pm} \rightarrow \mu^{ \pm}:$ & $0.12 \%$ & $\pi^{ \pm} \rightarrow \mu^{ \pm}:$ & $0.28 \%$ \\
\hline & \multicolumn{2}{|r|}{$\nu_{e}$} & \multicolumn{2}{|r|}{$\bar{\nu}_{e}$} \\
\hline Flux $\left(\nu \mathrm{POT}^{-1} \mathrm{~cm}^{-2}\right)$ & & $1.29 \times 10^{-13}$ & & $6.69 \times 10^{-14}$ \\
\hline Frac. of Total & & $0.40 \%$ & & $0.21 \%$ \\
\hline \multirow[t]{6}{*}{ Composition } & $K^{ \pm}$ & $42.43 \%$ & $K^{0}:$ & $49.87 \%$ \\
\hline & $\pi^{ \pm} \rightarrow \mu^{ \pm}:$ & $27.21 \%$ & $\pi^{ \pm} \rightarrow \mu^{ \pm}:$ & $33.63 \%$ \\
\hline & $K^{0}:$ & $25.77 \%$ & $K^{ \pm}:$ & $8.97 \%$ \\
\hline & $p(n):$ & $2.81 \%$ & $p(n):$ & $4.29 \%$ \\
\hline & $\pi^{ \pm}:$ & $1.50 \%$ & $\pi^{ \pm}:$ & $2.09 \%$ \\
\hline & Other: & $0.27 \%$ & Other: & $1.14 \%$ \\
\hline
\end{tabular}

Table 4.5: Predicted $\nu_{\mu} / \bar{\nu}_{\mu}$ (top) and $\nu_{e} / \bar{\nu}_{e}$ (bottom) fluxes at MiniBooNE detector while in beam off-target beam configuration. The contribution flux of the decay chains are provided for the primary parent meson production. 


\begin{tabular}{rccc}
\hline $\boldsymbol{\nu}$-Mode $\boldsymbol{\nu}$ Interaction & NUANCE $(\%)$ & $\mathrm{NCE}(\%)$ & $\mathrm{CCQE}(\%)$ \\
\hline $\mathrm{CCQE}$ & 40 & 2 & 84 \\
$\mathrm{CC} 1 \pi^{ \pm}$ & 24 & 1 & 13 \\
$\mathrm{NCE}$ & 17 & 76 & $<1$ \\
$\mathrm{NC} 1 \pi^{0}$ & 6 & 11 & $<1$ \\
$\mathrm{NC} 1 \pi^{ \pm}$ & 4 & 10 & $<1$ \\
$\mathrm{CC} 1 \pi^{0}$ & 4 & $<1$ & 2 \\
$\mathrm{Other}$ & 4 & $<1$ & $<1$ \\
\hline Off-Target: $\nu$ Interaction & $\mathrm{NUANCE}(\%)$ & $\mathrm{NCE}(\%)$ & $\mathrm{CCQE}(\%)$ \\
\hline $\mathrm{CCQE}$ & 42 & 4 & 84 \\
$\mathrm{CC} 1 \pi^{ \pm}$ & 20 & 1 & 13 \\
$\mathrm{NCE}$ & 19 & 77 & $<1$ \\
$\mathrm{NC} 1 \pi^{0}$ & 6 & 10 & $<1$ \\
$\mathrm{Other}$ & 6 & $<1$ & 1 \\
$\mathrm{CC} 1 \pi^{0}$ & 4 & $<1$ & 2 \\
$\mathrm{NC} 1 \pi^{ \pm}$ & 3 & 8 & $<1$ \\
\hline
\end{tabular}

Table 4.6: Breakdown of predicted $\nu_{\text {det }}$ by true neutrino interaction given by NUANCE. The first column gives the percentage of total events of each type, as determined at the primary vertex (not counting any final state interactions). The second and third columns gives the percentage of each event type for NCE and CCQE cuts. Other includes multiple $\pi$ production, deep inelastic scattering, elastic scattering off an electron, and delta radiative decay. 


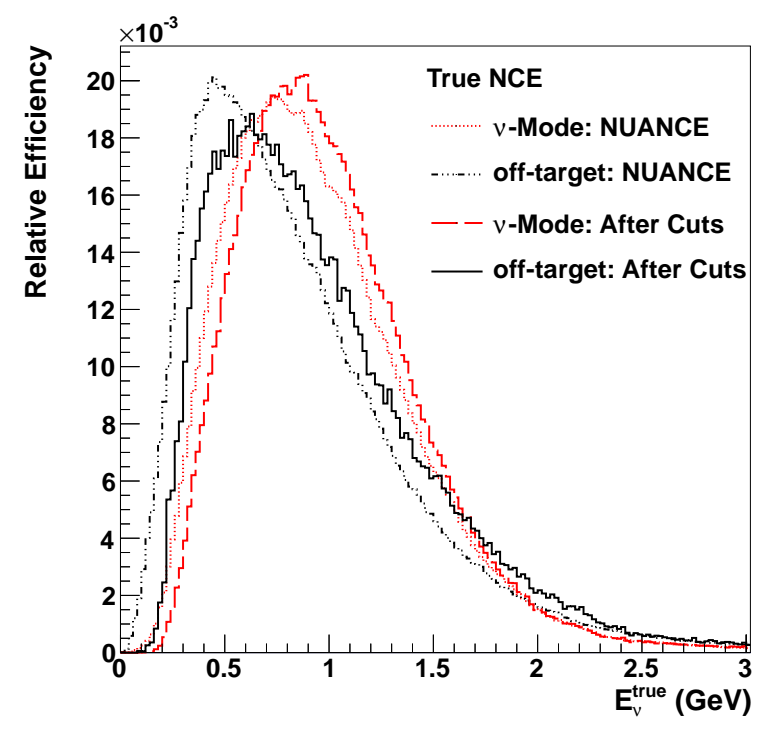

(a) NCE

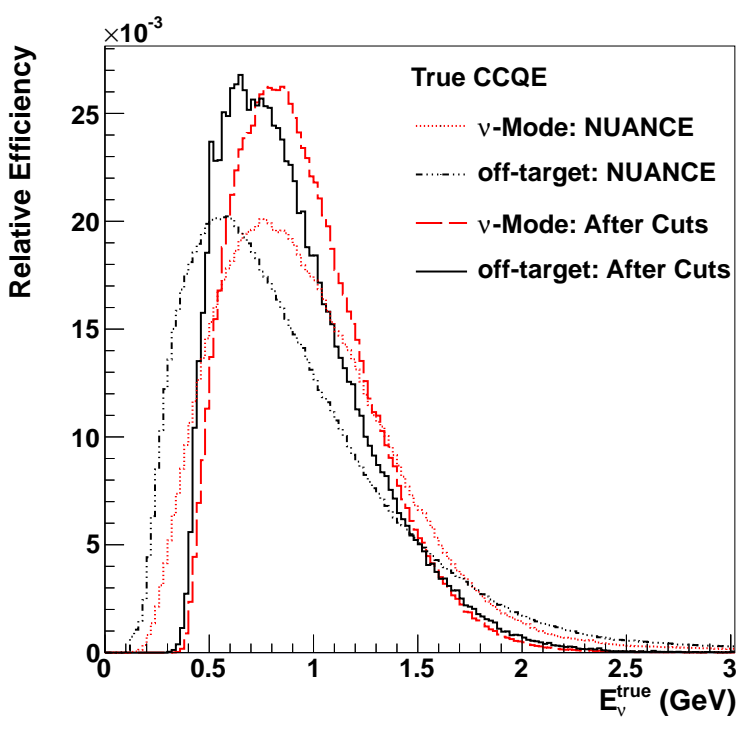

(b) CCQE

Figure 4.12: $E_{\nu}^{\text {true }}$ distributions for true (a) NCE and (n) CCQE events. The bold lines are after the corresponding selection cuts are made and the thinner lines are before detector effects and selection cuts. For each set $\nu$-Mode and off-target are given.

than the corresponding $\mathrm{NCE}_{\nu}$, but the difference is reduced in the CCQE distributions. The selection cuts reduces the sensitivity to the low energy events; CCQE has a harder low energy "cut" than NCE.

A key feature to understand the predicted number of DM events is knowing the total $\pi^{0}$ production. The average of the total $\pi^{ \pm}$, independent of if a neutrino was produced or not, distributions were used for the $\pi^{0}$ prediction because of the tuning of BooNEG4BEAM's $\pi^{ \pm}$distributions. Taking the average of $\pi^{+}$and $\pi^{-}$is consistent with the expected $\pi^{0}$ distribution $[85,86]$. For off-target $90 \%$ of the total pions are generated in the steel beam dump. Although the neutrino distribution changes depending on how the beam scrapes the target, the overall $\pi^{0}$ distribution remains constant over the off-target excursions because of the percentage of pions generated in the beam dump. Table 4.7 gives the break down of the material the $\pi^{ \pm}$were generated in for both off-target and $\nu$-Mode. Fig. 4.13 gives the 


\begin{tabular}{|c|c|c|}
\hline & $\pi^{+}$ & $\pi^{-}$ \\
\hline off-target meson/POT & 2.48 & 2.36 \\
\hline \multicolumn{3}{|l|}{ Composition } \\
\hline Air & $3.6 \%$ & $3.0 \%$ \\
\hline Aluminium & $0.2 \%$ & $0.2 \%$ \\
\hline Beryllium & $0.2 \%$ & $0.2 \%$ \\
\hline Concrete & $3.6 \%$ & $4.1 \%$ \\
\hline Dolomite & $0.1 \%$ & $0.1 \%$ \\
\hline Steel & $92.3 \%$ & $92.4 \%$ \\
\hline Neutrino Mode meson/POT & 2.54 & 2.51 \\
\hline \multicolumn{3}{|l|}{ Composition } \\
\hline Air & $1.7 \%$ & $1.4 \%$ \\
\hline Aluminium & $5.3 \%$ & $5.2 \%$ \\
\hline Beryllium & $29.5 \%$ & $27.6 \%$ \\
\hline Concrete & $28.0 \%$ & $27.6 \%$ \\
\hline Dolomite & $0.1 \%$ & $0.2 \%$ \\
\hline Steel & $35.4 \%$ & $38.0 \%$ \\
\hline
\end{tabular}

Table 4.7: The breakdown of the total number of $\pi^{ \pm}$per POT and by material in the beamline. A pion was counted if it had a total kinetic energy greater than $1 \mathrm{MeV}$, was traveling in the forward direction, and had a transverse momentum less than $1 \mathrm{GeV}$. Offtarget, in this table, refers to the nominal beam position measured by the multiwires, not the average of the four excursions that is used as the neutrino flux $\mathrm{CV}$. 
$\pi^{0}$ angle vs $\pi^{0}$ momentum 2D distribution for both $\nu$-Mode and off-target.

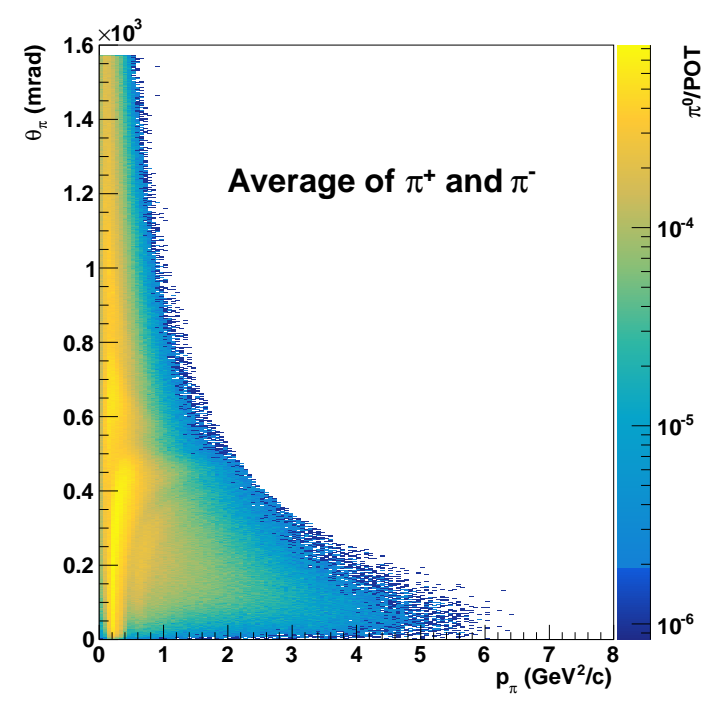

(a) Off-Target

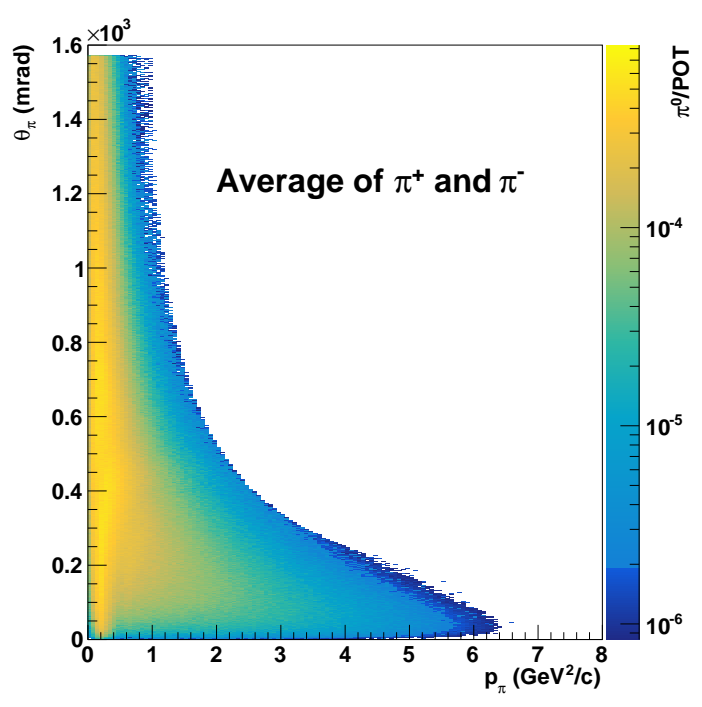

(b) $\nu$-Mode

Figure 4.13: The average of the $\pi^{ \pm}$produced in the BooNEG4BEAM simulation, used as the $\pi^{0}$ distribution in simulating DM, for off-target and $\nu$-Mode.

\subsubsection{TESTING CCQE $E_{\nu}^{\mathrm{QE}}$ PREDICTABILITY}

The off-target central-value flux was determined by looking at the integral number of CCQE

events. Can the CCQE $E_{\nu}^{\mathrm{QE}}$ distribution, with its distinct shape, rule out different excursions as candidate for the off-target flux? The following study looked to answer that question.

Define the smearing matrix between CCQE $E_{\nu}^{\text {true }}$ and $E_{\nu}^{\mathrm{QE}}$ as $\mathbf{M}$ so that for a given true distribution $\mathbf{S}$ the reconstructed $E_{\nu}^{\mathrm{QE}}$ distribution can be calculated by

$$
\mathbf{E}^{\prime}=\mathbf{S M}
$$

where $\mathbf{E}^{\prime}$ is a column matrix representing the predictive $E_{\nu}^{\mathrm{QE}}$ distribution.

Define a given neutrino flux output from BooNEG4BEAm by $\Phi\left(E_{\nu}\right)$. Modify the flux by $\Phi\left(E_{\nu}\right) \rightarrow \Phi\left(E_{\nu}\right)\left(1+b E_{\nu}\right)$ where $b$ is a floating parameter. For each $b, \mathbf{E}^{\prime}$ is calculated 
using Eq. (4.3), e.g. $S_{E_{\nu}=1 \mathrm{GeV}}=\Phi(1 \mathrm{GeV})[1+b(1 \mathrm{GeV})]$. To make the test shape only, the resulting $\mathbf{E}^{\prime}$ is scaled so the predicted number of CCQE events matches data. A fit was done using ROOT's implantation of MINUIT [87] to find the minimum $b$ for both the nominal off-target flux NBP and the off-target central-value flux $\overline{C V}$.

For a given $b$, the difference between the corresponding $\chi^{2}$ and the minimum $\chi^{2} \Delta \chi_{\min }^{2}$ was plotted as a function of $b$ to see how sensitive the off-target CCQE distribution is to $b$ for a given input flux. Fig. 4.14 shows $\Delta \chi_{\min }^{2}$ vs $b$ for both NBP and $\overline{C V}$. The scale used to

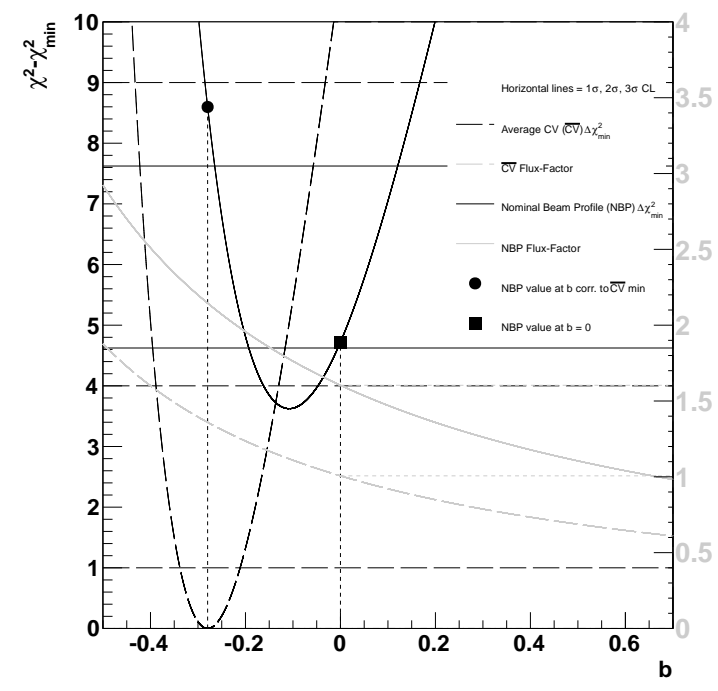

(a) No Systematics

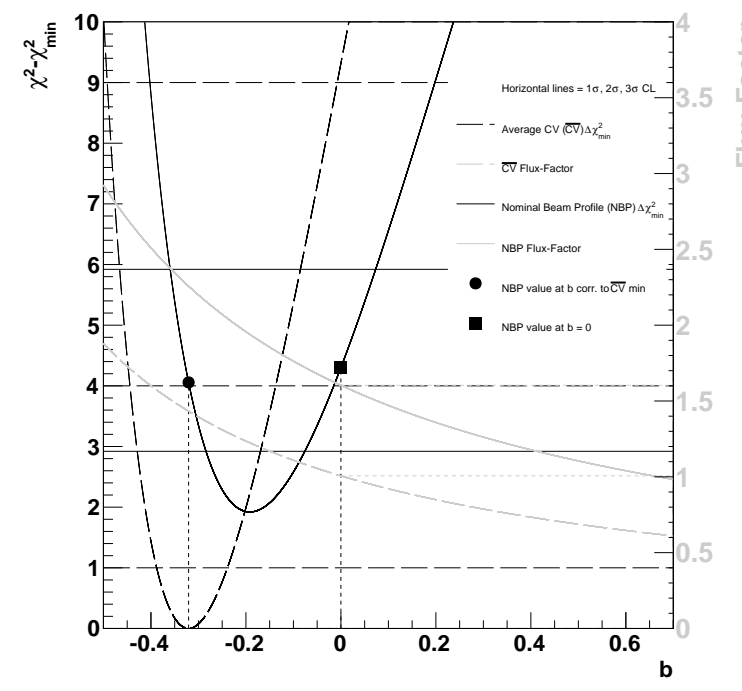

(b) With $15 \%$ Systematics

Figure 4.14: $\Delta \chi_{\min }^{2}$ distributions of the NBP flux compared to data (solid black curve) and $\overline{C V}$ flux compared to data (long dashed black curve) when varying $b$. The scale applied to $\mathbf{E}^{\prime}$ is shown for NBP (solid gray curve) and for $\overline{C V}$ (long dashed gray curve). The black square marker shows where on the NBP flux $\Delta \chi_{\text {min }}$ curve $b=0$ and the black circle marker shows where, on the same curve, $b=b_{\min _{\overline{C V}}}$. Horizontal gray dashed lines make it easier to see the flux-factor scale for NBP and $\overline{C V}$ when $b=0$. The scale for the flux-factors is given on the right while the scale for $\Delta \chi_{\min }^{2}$ is given on the left.

match the predicted and reconstructed number of CCQE events is also shown. The black 
markers on the plots show where on the NBP curve $b=0$ and $b=b_{\min _{\overline{C V}}}$ are located. The NBP default output, $b=0$, is practically at the minimum of the data curve, and the $\overline{C V}$ curve corresponds to less than $2 \sigma$ on the data curve.

The same analysis was performed assuming an uniform $15 \%$ uncorrelated systematic uncertainty on the flux. The results are shown in Fig. 4.14b. Including systematics reduces the number of sigma $\overline{C V}$ is away from NBP.

The study shows some sensitivity to the shape of the flux in the CCQE $E_{\nu}^{\mathrm{QE}}$ distribution, but no distinction between NBP, $\overline{C V}$, or any of the excursions can be made based on the $\mathrm{CCQE} E_{\nu}^{\mathrm{QE}}$ distribution. So for this analysis, the $\overline{C V}$ flux with systematic uncertainties, as described in the next section, were used.

\subsection{SYSTEMATIC UNCERTAINTY}

The results of the systematics study for neutrino oscillations was used for this analysis. See Refs. [82, 83, 88-90] for more information.

Systematic uncertainties for MiniBooNE are calculated with two different methods: 1) unisims, and 2) multisims. Unisims are generally used when the effect on the final physics parameters are changed, e.g. higher light yield, number and energy of pions produced change in beam, etc., while multisims are generally used when the covariance matrix of the input parameters is known. An unisim covariance matrix $M^{\text {unisim }}$ is generated by simulating a 1- $\sigma$ excursion around a particular input parameter, e.g. proton beam angle. The resulting uncertainty is given by

$$
M_{i j}^{\text {unisim }}=\left(N_{i}^{\text {unisim }}-N_{i}^{\mathrm{cv}}\right)\left(N_{j}^{\text {unisim }}-N_{j}^{\mathrm{cv}}\right)
$$

where $N_{i}^{\mathrm{unisim} / \mathrm{cv}}$ is the number of events predicted for the ith bin by the excursion (unisim) or by the central value simulation $(\mathrm{CV})$. The resulting $M_{i j}^{\text {unisim }}$ is assumed to be a 1- $\sigma$ uncertainty around the central value. The resulting covariance matrix for each unisim is added 
together. Unisims considered in this analysis were beam unisims, effects of the discriminator and effects of the QT boards, uncertainty on POT counting, previous trigger accidental efficiency, scaling the STROBE triggers, and uncertainty in the dirt contribution $[76,82]$.

The unisim method falls short if there are correlations between parameters. The multisim method handles correlations between parameters because it takes as input a covariance matrix of certain parameters. The following is an example input covariance matrix for the cross section uncertainties

$$
M_{x s e c}^{\text {input }}=\left[\begin{array}{ccc}
\operatorname{var}\left(M_{A}\right) & \operatorname{cov}\left(M_{A}, \kappa\right) & 0 \\
\operatorname{cov}\left(M_{A}, \kappa\right) & \operatorname{var}(\kappa) & 0 \\
0 & 0 & \operatorname{var}(\Delta s)
\end{array}\right]
$$

where $\Delta s$ is the strange contribution to the proton spin. Several trials are run to see how the reconstructed distribution changes by picking different parameter values based off the input covariance matrix. This is possible because the analytic and/or simulated relationship between the input parameters and the true energy of the event is known. The covariance matrix from the multisim method was calculated by

$$
M_{i j}^{\text {multisim }}=\frac{1}{\# \text { trials }} \sum_{s=1}^{\# \text { trials }}\left(N_{i}^{s}-N_{i}^{\mathrm{cv}}\right)\left(N_{j}^{s}-N_{j}^{\mathrm{cv}}\right) .
$$

Multisims considered in this analysis were cross section, hadronic interactions, and changes in pion and kaon production due to uncertainties in the Sanford-Wang distribution parameters. Although the optical properties of the mineral oil were well studied, changes in the parameters had complex changes in the results.

The optical model uncertainty was handeled differently than the other multisim systematics. Other multisim systematics reweighted the CV based off a ratio of the new to original true energy. New simulations were not needed allowing statistical uncertainty to be ignored. Adjusting the optical model however causes the range of energy, the timing, amount of Cherenkov verses scintillation light, etc. to be different than original, thus affecting the reconstruction output, and removing the ability to analytically reweight previously 
run events. About 70 sets of simulations were run where each set was a change in the optical model properties based off measurements, see Sec. 3.2.3. These sets of simulations were run with lower statistics and therefore statistical uncertainty had to be considered in generating the systematic covariance matrix. Eq. (4.4) was still used to calculate the final uncertainty since a given set of parameters was chosen based on the correlation matrix of the optical model parameters.

The final covariance matrix is then a simple sum of all the covariance matrices generated, where each category of unisims and multisims are considered independent.

\subsubsection{OFF-TARGET SYSTEMATICS}

Correlations were calculated between different $Q_{Q E}^{2}$ bins and between different data sets.

Fig. 4.15 shows correlation matrices for the cross section and optical model uncertainties.

For the cross section and optical model uncertainties, the expectation was to see corre-

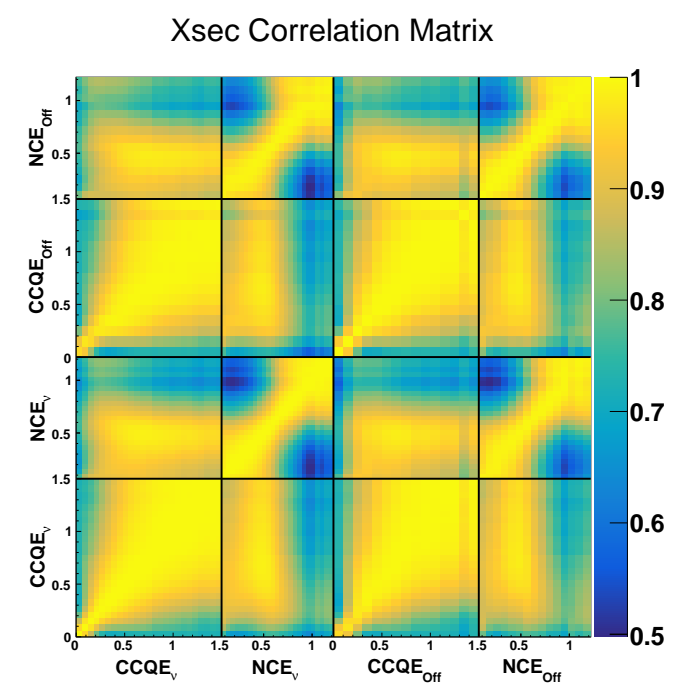

(a) Cross-Section

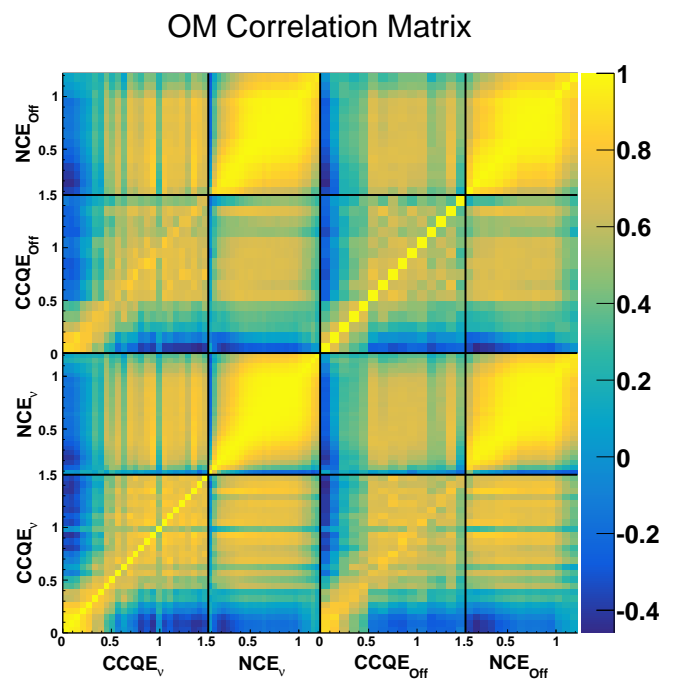

(b) Optical Model

Figure 4.15: Example of the individual correlation matrices from the systematic uncertainty studies. Cross-section and Optical Model systematics are shown. All four distributions are plotted as functions of $Q_{Q E}^{2}$ 
lations between data sets of the same channel type, e.g. if an increase in a parameter increases the number of events for $\mathrm{NCE}_{\nu}$, it increases for $\mathrm{NCE}_{\mathrm{Off}}$, and Fig. 4.15 shows that. The total correlation matrix is given in Fig. 4.16, with the breakdown of each systematic for each distribution given in Table 4.8. The total systematic uncertainties are domi-

\section{Total Correlation Matrix}

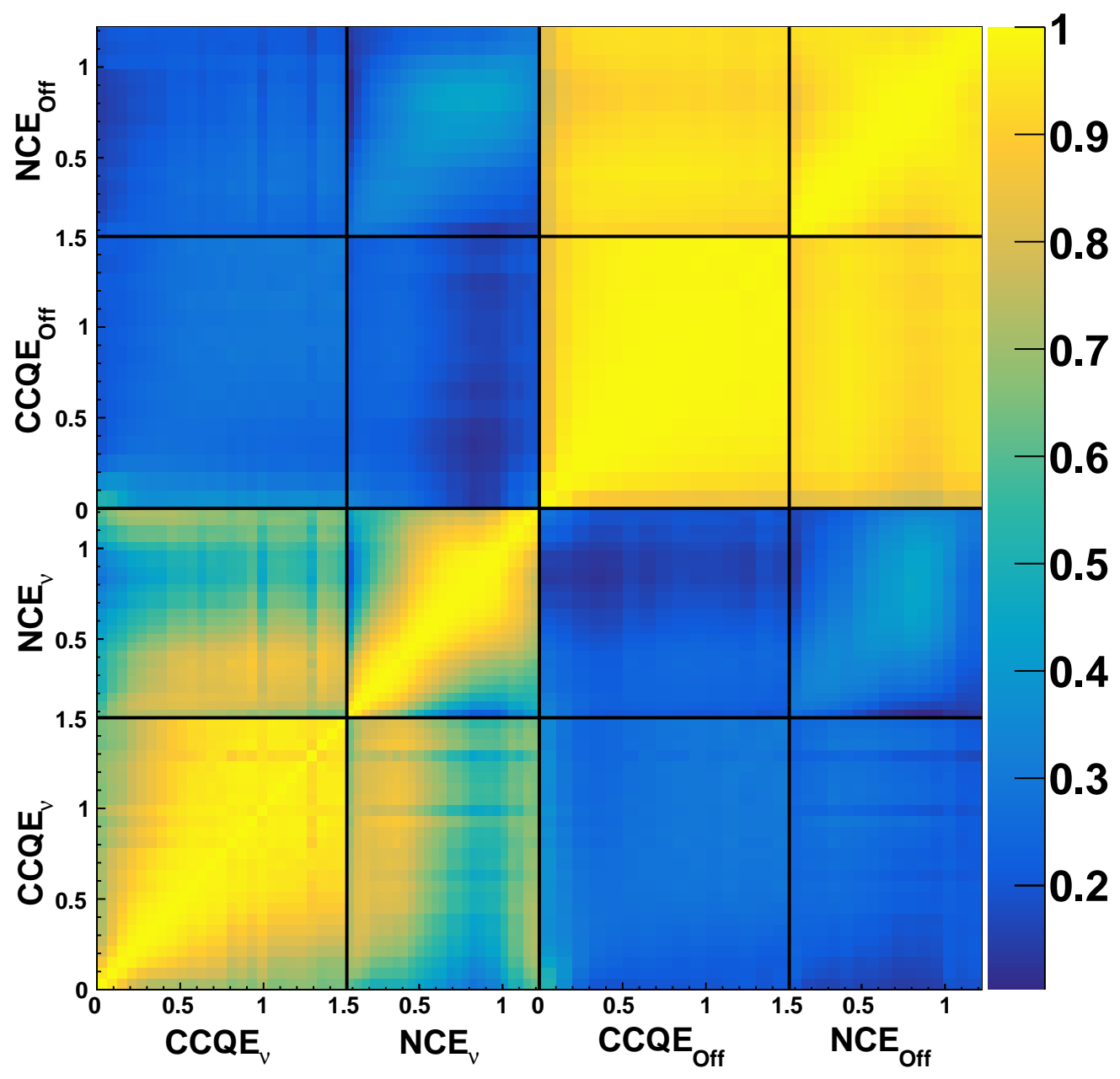

Figure 4.16: The energy dependent systematic uncertainty correlation matrix between the four datasets.

nated by cross section, optical model and flux uncertainties. The flux and cross section uncertainties contain a dominate overall scaling term, i.e. number of events all go up or 


\begin{tabular}{|c|c|c|c|c|}
\hline Systematic & $\mathrm{CCQE}_{\nu}$ & $\mathrm{NCE}_{\nu}$ & $\mathrm{CCQE}_{\mathrm{Off}}$ & $\mathrm{NCE}_{\mathrm{Off}}$ \\
\hline \multicolumn{5}{|l|}{ Cross-sections } \\
\hline Cross-Section & 16.6 & 13.3 & 18.2 & 7.9 \\
\hline Hadronic & $<0.1$ & $<0.1$ & $<0.1$ & $<0.1$ \\
\hline \multicolumn{5}{|l|}{ Detector Model } \\
\hline Discriminator and QTCorr & 1.4 & 2.0 & 1.3 & 1.5 \\
\hline Optical Model & 2.3 & 13.2 & 2.1 & 8.1 \\
\hline Dirt & $<0.1$ & 2.2 & $<0.1$ & 1.0 \\
\hline POT & 1.9 & 1.6 & 1.9 & 0.9 \\
\hline \multicolumn{5}{|l|}{$\nu$ Flux } \\
\hline Beam Unisims & 4.3 & 4.0 & 50.5 & 31.3 \\
\hline$K^{-}$Prod & $<0.1$ & $<0.1$ & 0.3 & 0.2 \\
\hline$K^{0}$ Prod & $<0.1$ & $<0.1$ & 0.1 & 0.1 \\
\hline$K^{+}$Prod & 0.2 & 0.5 & 0.8 & 0.6 \\
\hline$\pi^{-}$Prod & 0.2 & 0.1 & 2.7 & 1.0 \\
\hline$\pi^{+}$Prod & 4.0 & 3.8 & 3.5 & 2.6 \\
\hline \multicolumn{5}{|l|}{ Misc. } \\
\hline Strobe Scale & 0.0 & 1.3 & 0.0 & 0.2 \\
\hline Previous Trigger Cut & 0.0 & 0.2 & 0.0 & 0.1 \\
\hline Total Systematic & 17.9 & 19.9 & 54.0 & 33.5 \\
\hline Statistical & 0.3 & 0.3 & 3.2 & 2.6 \\
\hline
\end{tabular}

Table 4.8: Percent systematic uncertainty on total number of events for each channel and beam-mode. 
go down together. These dominant uncertainties drive the total correlation matrix to have no negative correlations and the large uncertainty on the off-target flux drives all $\mathrm{CCQE}_{\mathrm{Off}}$ and $\mathrm{NCE}_{\mathrm{Off}}$ bins to be almost fully correlated.

The covariance matrix could be calculated either around the CV or around the mean of the multisims. The latter method would then require the resulting uncertainty to be scaled to the CV. For this analysis the former was chosen to be consistent with the oscillation analysis. The former will almost always produce larger uncertainty than the latter. To keep correlations between data sets, the multisim random number seeds were chosen to be the same between those data sets.

In the published $\mathrm{CCQE}_{\nu}$ [69] and $\mathrm{NCE}_{\nu}$ [50] cross section papers, the cross section uncertainties did not include the true uncertainty on the CCQE or NCE events respectively because the cross section for these channels were measured and not subject to simulation (MC) predicted signal uncertainties. With all neutrino interactions treated as background for this analysis the uncertainties on true CCQE and $\mathrm{NCE}$ events were included in all samples.

As stated in Sec. 3.2.3, the default $M_{A}^{\mathrm{eff}}$ and $\kappa$ values used to run the simulation were $1.23 \pm 0.20$ and $1.019 \pm 0.011$, respectively. The measured values from $\mathrm{CCQE}_{\nu}$ were $M_{A}^{\mathrm{eff}}=$ $1.035 \pm 0.170$ and $\kappa=1.007 \pm 0.009$ [83]. The updated values were used in this analysis, so in calculating the uncertainty in the cross section the central value was set to the new values and the input covariance matrix was updated to include the uncertainty in those values. The off-target beam unisims do not include the unisim cross section uncertainties used in $\nu$-Mode because the uncertainties from interactions in aluminum and beryllium are small with the increased percentage of neutrinos coming from the air and steel, and the position unisims dominate the uncertainty in off-target running.

$\mathrm{NCE}_{\mathrm{Off}}$ has smaller systematic uncertainty compared to both $\mathrm{NCE}_{\nu}$ and $\mathrm{CCQE}_{\mathrm{Off}}$ because the larger percentage of BUB which is known statistically to an order of a percent 
for off-target running. $\mathrm{CCQE}_{\mathrm{Off}}$ peaks at a lower $E_{\nu}^{Q E}$ value than $\mathrm{CCQE}_{\nu}$, which drives the cross section uncertainty in $\mathrm{CCQE}_{\mathrm{Off}}$ to be higher than $\mathrm{CCQE}_{\nu}$. The kaon and pion production uncertainties have the same underlying covariance matrix for both $\nu$-Mode and off-target.

\subsection{FINAL $Q_{Q E}^{2}$ DISTRIBUTIONS}

Now, with the selection criteria for each sample defined (see Sections 4.1.1 and 4.1.2), the predicted neutrino flux determined, and the correlated uncertainties set, the four distribution as a function of $Q_{Q E}^{2}$ are shown in Fig. 4.17. Each plot shows the predicted or measured number of events/(1e20POT) to account for the different number of POT in $\nu$-Mode and off-target samples. The data is shown with statistical uncertainty bars and the predicted background is shown with systematic uncertainty bars. The breakdown of the backgrounds is also shown.

The CCQE distributions have a very small component of dirt; small enough to be ignored as was done in the $\nu$-Mode analysis [69]. Fig. 4.17a shows the BUB is a high percentage of the background at low $Q_{Q E}^{2}$ for $\mathrm{NCE}_{\mathrm{Off}}$ compared to $\mathrm{NCE}_{\nu}$. For $\mathrm{NCE}_{\mathrm{Off}}$, 1579 events $\pm 33.5 \%$ (sys.) were predicted and 1465 events $\pm 2.6 \%$ (stat.) were observed. There is a slight deficit but consistent with prediction.

Fig. $4.17 \mathrm{~b}$ shows the total background prediction is high in every bin compared to data. It is hypothesized that either the cross section of NCE events or NCE-like events is wrong in NUANCE. For $\nu$-Mode and $\bar{\nu}$-Mode analyses, this was not addressed as the goal of the analysis was a measurement of data, not a model/data comparison. For this analysis; however, if no correction is made then the resulting confidence limit may result in a stronger confidence limit than reality. The correction that is made is discussed in Sec. 5.2.

Table 4.9 gives the number of predicted and observed events. Although the off-target

flux is reduced by a factor of 26.6 (see Sec. 4.2.2), the CCQE and NCE event rate is 


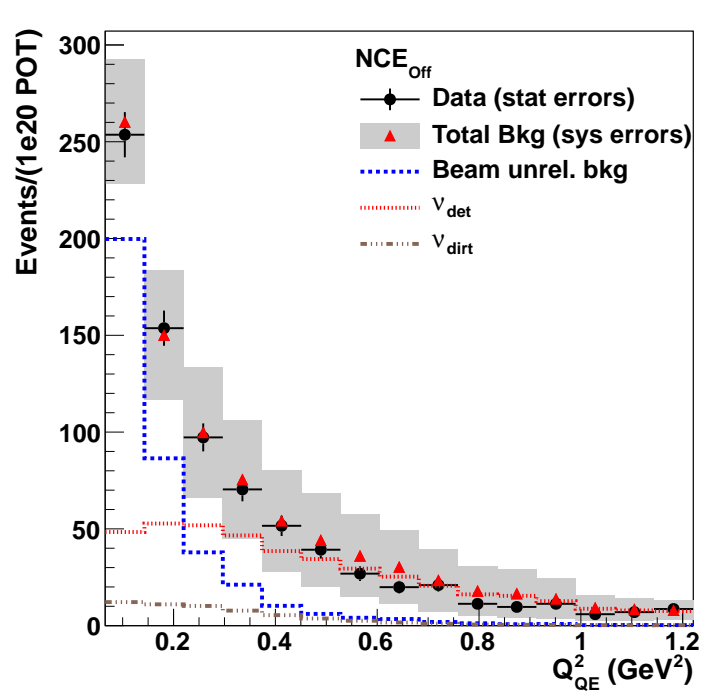

(a) $\mathrm{NCE}_{\text {Off }}$

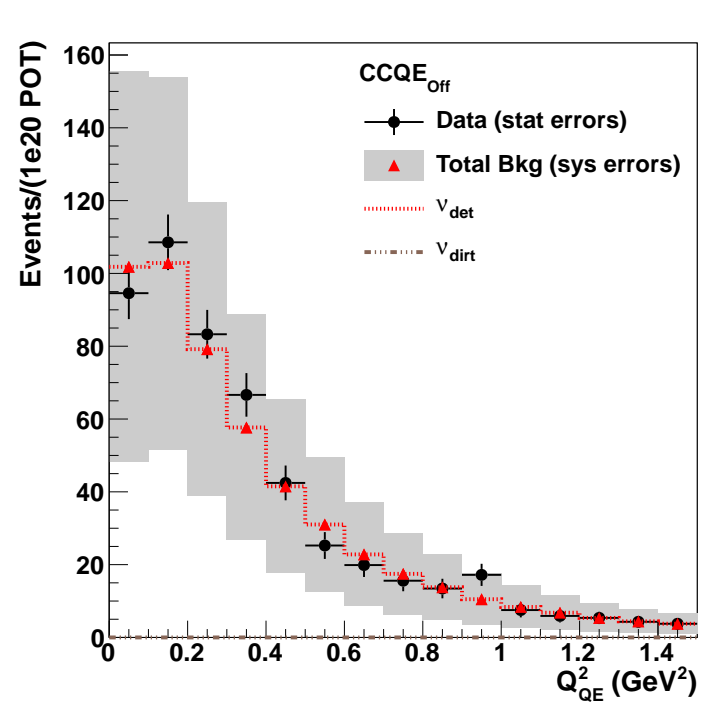

(c) $\mathrm{CCQE}_{\mathrm{Off}}$

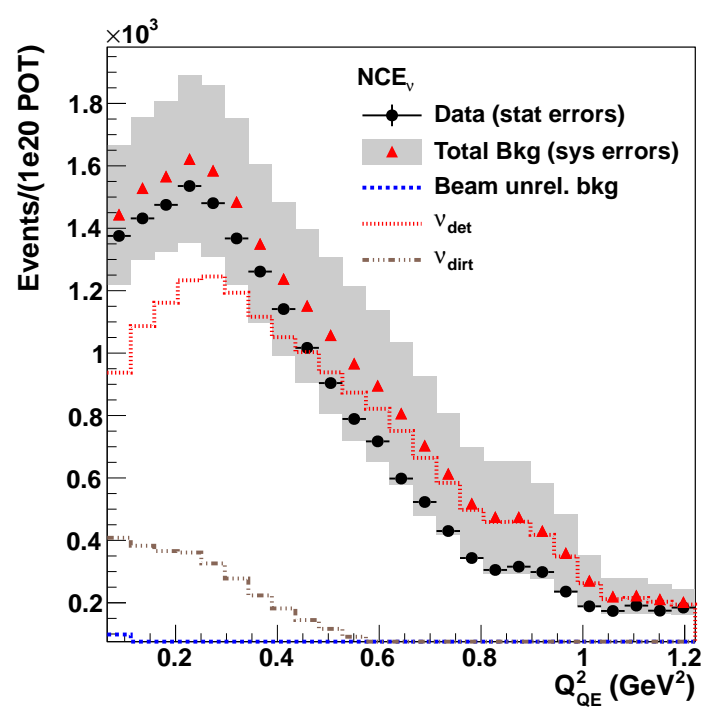

(b) $\mathrm{NCE}_{\nu}$

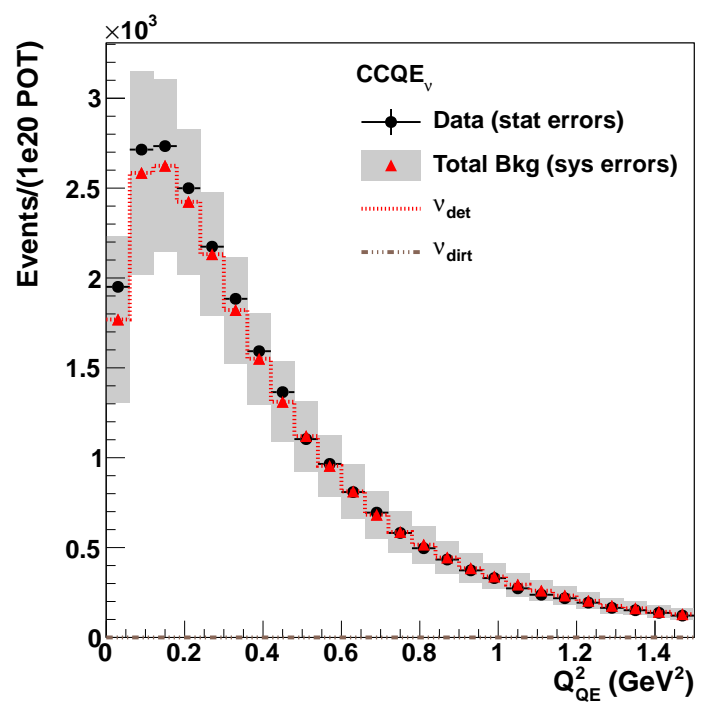

(d) $\mathrm{CCQE}_{\nu}$

Figure 4.17: The NCE/CCQE $\nu$-Mode/off-target distributions. Statistical uncertainty are shown in black and systematic uncertainty are shown in gray. 


\begin{tabular}{|c|c|c|c|c|}
\hline \multirow[b]{2}{*}{$\mathrm{NCE}$} & \multicolumn{2}{|c|}{ Off-Target } & \multicolumn{2}{|c|}{$\nu$-Mode } \\
\hline & \# events & uncertainty & \# events & uncertainty \\
\hline exp. data & 1465 & 38 & 119255 & 354 \\
\hline \multicolumn{5}{|l|}{ pred. backgounds } \\
\hline beam-unrelated & 697 & 11 & 1819 & 1821 \\
\hline$\nu_{\mathrm{det}}$ & 775 & 454 & 20591 & 3645 \\
\hline$\nu_{\text {dirt }}$ & 107 & 81 & 115854 & 26067 \\
\hline Total background & 1579 & 529 & 138264 & 27514 \\
\hline Propagated Fit Results & 1548 & 198 & 133011 & 14422 \\
\hline Constrained Fit Results & 1454 & 101 & 119246 & 345 \\
\hline CCQE & \# events & uncertainty & \# events & uncertainty \\
\hline exp. data & 956 & 31 & 156340 & 395 \\
\hline \multicolumn{5}{|l|}{ pred. backgounds } \\
\hline$\nu_{\mathrm{det}}$ & 944 & 510 & 152875 & 27365 \\
\hline$\nu_{\text {dirt }}$ & 0.011 & 0.015 & 1.8 & 0.3 \\
\hline Total background & 944 & 510 & 152877 & 27365 \\
\hline Propagated Fit Results & 999 & 233 & 162042 & 19718 \\
\hline Constrained Fit Results & 950 & 31 & 156327 & 395 \\
\hline
\end{tabular}

Table 4.9: The integral number of events for each distribution. The experimental data, total background, breakdown of background, and fit results are given. The fit results are discussed in Sec. 5.2. 
decreased by a factor of 47.6, after adjusting for the difference in off-target and $\nu$-Mode POT. The difference is due to the softer flux combined with the NCE and CCQE cross sections decreasing with lower $E_{\nu}^{\text {true }}$. 


\section{CHAPTER 5}

\section{FIT PROCEDURE}

The purpose of a fit the data distributions described in the previous chapter is to find the best estimate of the amount of dark matter $(\mathrm{DM})$ in the $\mathrm{NCE}_{\mathrm{Off}}$ sample given experimental uncertainties. To best use the constraining information in the other MiniBooNE samples is to use a simultaneous combined fit of all four distributions, referred to as chain in this discussion. The term chain came from showing the distributions in one plot where the distributions were put together in a chain, as can be seen in Fig. 5.1a, which made keeping track of the systematic correlations easier. The correlation matrix for the fit is shown in Fig. 4.16. Another viable fitting method is to take a double ratio, $\mathrm{NCE}_{\mathrm{Off}} \mathrm{CCQE}_{\nu} /\left(\mathrm{NCE}_{\nu} \mathrm{CCQE}_{\mathrm{Off}}\right)$, as a function of $Q_{Q E}^{2}$. The data compared to background prediction after taking the ratio is shown in Fig. 5.1b. The full covariance matrix is needed for both methods. An advantage the chain method has over the ratio is that each distribution in the chain does not have to have the same independent variable, or the same number of bins. This chapter explains how fake data sets get generated, the results from the background only and model independent fits.

\subsection{GENERATING FAKE DATA}

Fake data sets are used throughout the discussion of fits and when setting confidence limit (see Sec. 6.2.2). This section will discuss how the fake data sets are generated. Fake data 


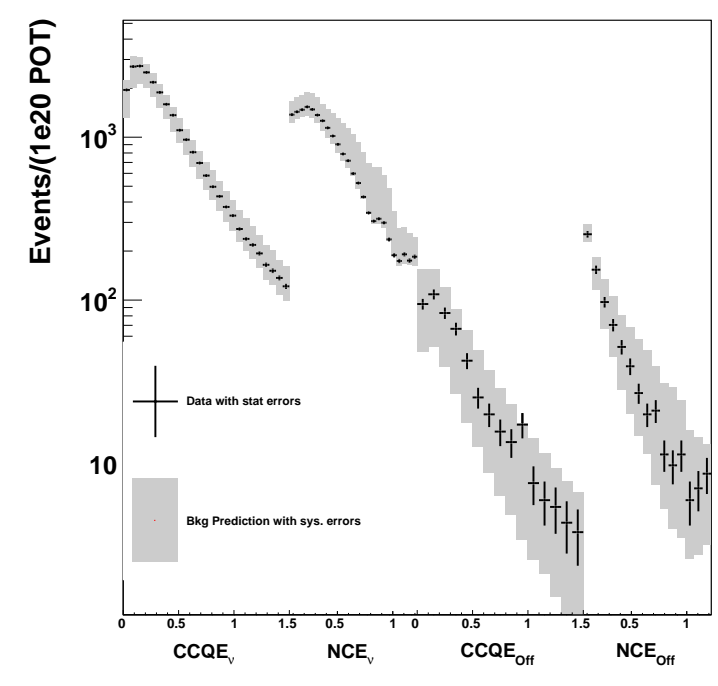

(a) Chain

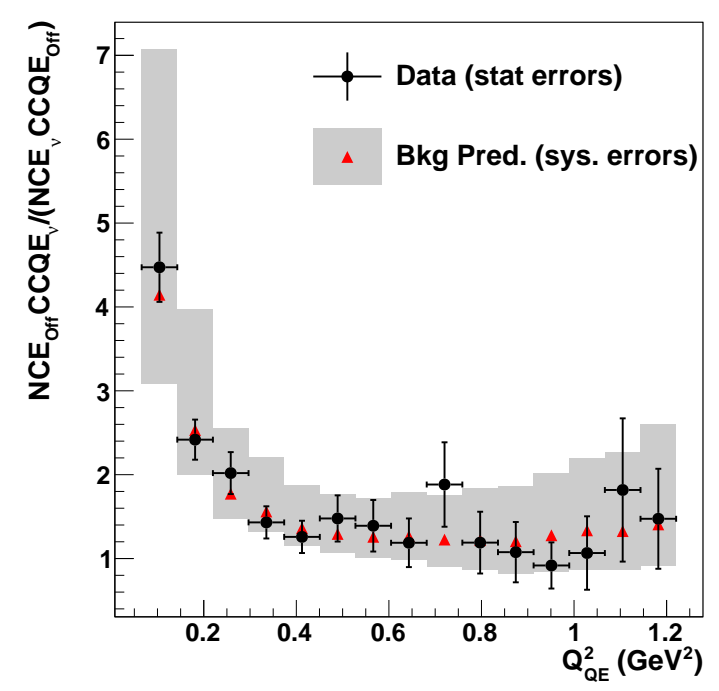

(b) Ratio

Figure 5.1: The data and total background predicted distributions for $\mathrm{CCQE}_{\nu}, \mathrm{NCE}_{\nu}$, $\mathrm{CCQE}_{\mathrm{Off}}, \mathrm{NCE}_{\mathrm{Off}}$ as a (a) chain and (b) ratio in $Q_{Q E}^{2}$

is generated around a given central value and the corresponding covariance matrix, see Fig. 5.2 for a schematic of the process.

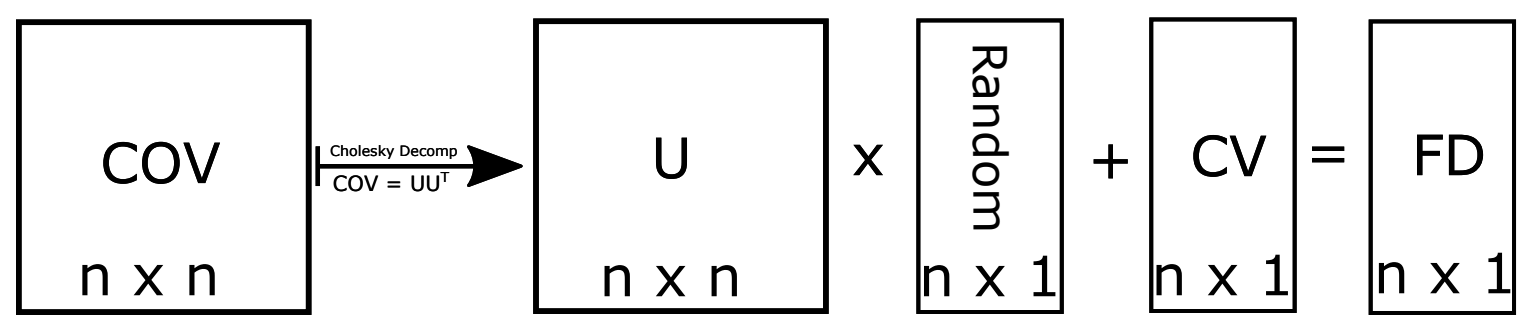

Figure 5.2: Schematic of how to generate fake data

The covariance matrix is decomposed by Cholesky decomposition [91, 92], $M=L L^{\top}$. Depending on the definition used, the result is an upper, or lower triangular matrix. For each bin in the distribution an uncorrelated random number is generated. The random numbers were generated from a Gaussian distribution with a mean of zero and a standard deviation of one. Multiplying the triangular matrix by the vector of uncorrelated random numbers produced a vector of correlated random numbers. The resulting fake data set is 
generated by adding the correlated random numbers to the central value distribution. If any element in fake data is negative it is set to zero because number of events has a lower bound of zero.

To produce $N$ fake data sets, $N$ uncorrelated sets of random numbers are generated. Each set is multiplied by the triangular matrix giving $N$ fake data sets whose elements are correlated.

\subsection{BACKGROUND-ONLY FIT}

A background-only fit was performed to determined how predicted background compares to data, and determine nuisance parameters. The six nuisance parameters used in this analysis are summarized in Table 5.1. The $\nu$-Mode and off-target flux factors are to allow

\begin{tabular}{ll}
\hline Symbol & Name \\
\hline$f_{\nu}$ & $\nu$-Mode Flux Factor \\
$f_{\text {Off }}$ & off-target Flux Factor \\
$y_{0}$ & neutral-current elastic (NCE) cross section offset \\
$\mathcal{A}$ & NCE cross section Gaussian amplitude \\
$x_{0}$ & NCE cross section Guassian energy off-set \\
$\sigma$ & NCE cross section Guassian energy uncertainty \\
\hline
\end{tabular}

Table 5.1: The symbol and name of the nuisance parameters used for the analysis.

the integral number of events predicted by the simulation (MC) to be adjusted, within uncertainties and beyond the initial tuning, to better match the data. The other four nuisance parameters are parameters of the following equation

$$
G(x)=y_{0}-\mathcal{A} \exp \left[\frac{-\left(x-x_{0}\right)^{2}}{2 \sigma^{2}}\right] \text {. }
$$

The purpose of these nuisance parameters was to adjust the $\mathrm{NCE}_{\nu}$ prediction to data, within uncertainties. The Gaussian correction to $\nu_{\text {det }}$, as shown in Fig. 5.3, matches to 
data well. No distinction is made between true NCE and NCE-like events because they are

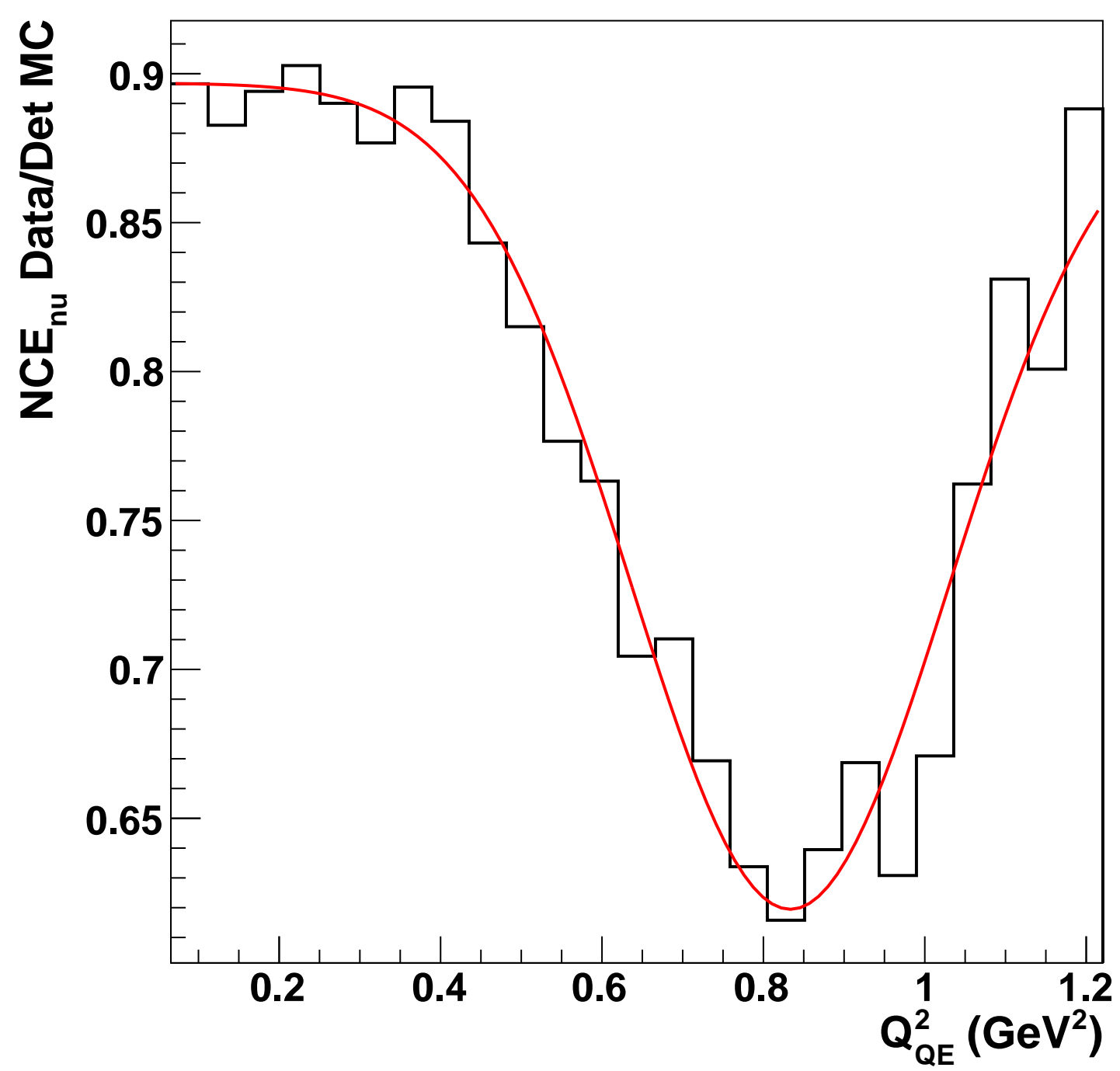

Figure 5.3: The ratio of $\mathrm{NCE}_{\nu}$, after beam-unrelated background (BUB) and $\nu_{\text {dirt }}$ subtraction, data to $\nu_{\text {det }}$ as a function of $Q_{Q E}^{2}$ (black histogram) with fit results of the fit to Eq. (5.1) (red line).

both backgrounds for this analysis and can be combined. 
The nuisance fit parameters in Table 5.1 are incorporated into the fit function by

$$
N_{i}^{\mathrm{Pred}^{\prime}}=\left\{\begin{array}{ll}
\mathrm{CCQE}_{\nu_{i}}^{\mathrm{MC}} / f_{\nu} & i \in \mathrm{CCQE}_{\nu}, \\
\left(\mathrm{NCE}_{\nu_{i}}^{\operatorname{detMC}} * G\left(x_{i}\right)+\mathrm{NCE}_{\nu_{i}}^{\text {dirtMC }}\right) / f_{\nu}+\mathrm{NCE}_{\nu_{i}}^{\mathrm{BUB}} & i \in \mathrm{NCE}_{\nu} \\
\mathrm{CCQE}_{\mathrm{Off}_{i}}^{\mathrm{MC}} / f_{\mathrm{Off}} & i \in \mathrm{CCQE}_{\mathrm{Off}} \\
\left(\mathrm{NCE}_{\mathrm{Off}_{i}}^{\text {detMC }} * G\left(x_{i}\right)+\mathrm{NCE}_{\mathrm{Off}_{i}}^{\text {dirtMC }}\right) / f_{\nu}+\mathrm{NCE}_{\mathrm{Off}}^{\mathrm{BUB}} & i \in \mathrm{NCE}_{\mathrm{Off}}
\end{array},\right.
$$

where $i$ is the bin number in the chain; $x_{i}$ corresponds to the $Q_{Q E}^{2}$ value for the $i$ th bin, and $N_{i}^{\mathrm{X}}$ is the number of events predicted/measured for the $\mathrm{X}$ background, where $\mathrm{X}$ can be $\nu_{\text {det }}, \nu_{\text {dirt }}$ or BUB.

The fit uses MINUIT to minimize the six nuisance parameter space to calculate the nuisance parameter covariance matrix. The initial guess passed to MINUIT is calculated for $f_{\nu}\left(f_{\mathrm{Off}}\right)$ by setting them to the ratio of prediction to data for $\mathrm{CCQE}_{\nu}\left(\mathrm{CCQE}_{\mathrm{Off}}\right)$. The NCE/NCE-like cross section adjustment parameters are generated by subtracting BUB and $\nu_{\text {dirt }}$ from $\mathrm{NCE}_{\nu}$ data and then fitting the $Q_{Q E^{-}}^{2}$ dependent ratio of data to $\nu_{\text {det }}$ with Eq. (5.1), (see Fig. 5.3). The initial guess for the flux factors does not include correlations between the bins while the NCE/NCE-like cross section adjustment does use the diagonal bins of the systematic covariance matrix in the fit. Table 5.2 gives the initial and fitted values for the nuisance parameters.

The fit method uses a log-likelihood test statistic [93] given by

$$
-2 \ln (\mathcal{L})=\chi^{2}+\ln (|M|),
$$

where

$$
\chi^{2}=\Delta^{\top} M^{-1} \Delta, \Delta_{i}=N_{i}^{\text {Data }}-N_{i}^{\text {Pred }^{\prime}}
$$

is the standard correlated $\chi^{2}$ test statistic. The underlying probability distribution used for the likelihood is given by a correlated multiparameter Gaussian probability distribution function instead of the Poisson distribution needed for low count experiments. With an initial guess at what the nuisance parameters are expected to be, Eq. (5.3) can be expanded 


\begin{tabular}{ccrr}
\hline Fit Param & Initial Guess & Result & Uncertainty \\
\hline$f_{\nu}$ & 0.98 & 0.94 & 0.11 \\
$f_{\text {Off }}$ & 0.99 & 0.95 & 0.22 \\
$y_{0}$ & 0.90 & 0.98 & 0.08 \\
$\mathcal{A}$ & 0.27 & 0.27 & 0.13 \\
$\sigma$ & 0.20 & 0.25 & 0.04 \\
$x_{0}$ & 0.83 & 0.89 & 0.04 \\
$-2 \ln (\mathcal{L})$ & & 442.0 & \\
$\ln (|M|)$ & & 393.9 & \\
$\chi^{2} / N D F$ & & $48.1 / 74$ & \\
\hline
\end{tabular}

Table 5.2: The numerical values of the nuisance parameters from the initial guess and the background-only fits shown in Fig. 5.4.

to

$$
-2 \ln (\mathcal{L})=\chi^{2}+\ln (|M|)+\sum_{i}^{n_{\text {params }}} \frac{\left(p_{i}-p_{i}^{\text {init }}\right)^{2}}{\sigma_{p_{i}^{\text {init }}}^{2}},
$$

where $p_{i}$ is the $i$ th fit parameter, $p_{i}^{\text {init }}$ is the initial guess on the $i$ th fit parameter, and $\sigma_{p_{i}^{i n i t}}$ is the uncertainty on the initial guess for the $i$ th parameter. Since the flux factors should be independent of the correlations between bins, Eq. (5.4) is used instead of Eq. (5.3). The difference between using Eq. (5.3) and Eq. (5.4) is small and consistent with each other. Eq. (5.4) was used over Eq. (5.3) to include information from the initial guess.

When using Eq. (5.4), the only nuisance parameters that are constrained are $f_{\nu}$ and $f_{\text {Off }}$ and the uncertainty on the initial guess is set to $30 \%$ of the initial guess to ensure the parameters do not wander off to silly values.

During the fit the fractional covariance matrix

$$
F_{i j}=\frac{M_{i j}}{N_{i}^{\text {Pred }} N_{j}^{\text {Pred }}}
$$

where $M$ is the covariance matrix (described in Sec. 4.3) was held constant. This was done for the oscillation analysis [93] and is achieved in an iterative method where the covariance 
matrix was held constant while the nuisance parameters were determined using MINUIT. The covariance matrix was then adjusted to the results of the nuisance parameters; the fit was performed again. This pattern repeats itself until the change in the test statistic is less than a percent.

Fig. 5.4 shows the fit compared to data and predicted background, and the resulting nuisance parameters are given in Table 5.2. The strong correlations between energy bins

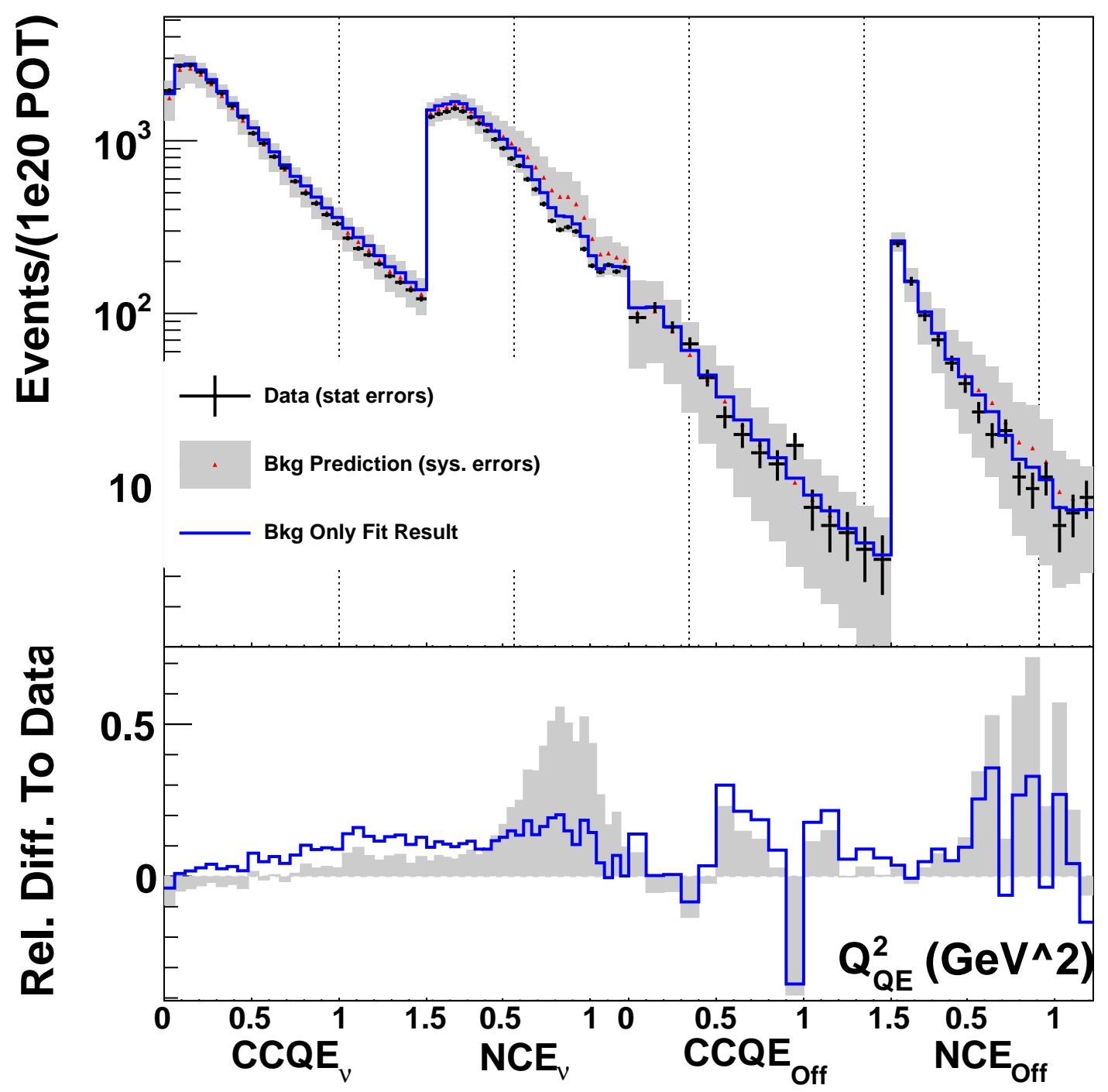

Figure 5.4: The results of the background-only fit.

and between data sets are driving the fits to a best value that is somewhat counterintuitive, 
i.e failed the "eye" test. It can be shown that if the correlations are strong then the fit between two points can be either above or below both points. A test case of this is given in appendix B. A fit using the singular value decomposition of the covariance matrix was done to see how the results from the fit compare in an uncorrelated basis. The singular value decomposition, as explained in more detail in appendix $\mathrm{C}$, rotates a correlated matrix to an uncorrelated basis. The fit results are more intuitive when viewed in the uncorrelated basis, i.e. the "eye" test works.

\subsubsection{PROBABILITY OF FIT}

Based off the $\chi^{2}$-test statistic, there is a $0.1 \%$ chance to get the small $\chi^{2}$ value obtained from the background-only fit - the fit is too good. Fig. 5.5 shows that comparing with fake data the probability is about $3 \%$ - fit is still too good. Fitting the $Q_{Q E}^{2}$ distribution with an upper limit of $0.6 \mathrm{GeV}^{2}$ produces a $\chi^{2}$ fit probability of $11 \%$. The high $Q_{Q E}^{2} \mathrm{NCE}$ optical model uncertainties are driving the lower $\chi^{2}$ value, suggesting that the optical model uncertainty is conservative. Due to the conservative nature of the systematics, the resulting confidence limits will be conservative. The low $3 \%$ probability of the test statistic can be small due to the MC being tuned to previous MiniBooNE data. The next section describes an analytical fit that further constrains the MC to data to see what the true probability of the fit is.

\section{CONSTRAINED FIT}

A constrained fit was done by applying the nuisance parameters from the background-only fit to determine how probable the background-only fit is knowing the $\mathrm{MC}$ was tuned by

previous MiniBooNE measurements. All calculations are done in units of events/1e20 protons on target (POT). Denoting the number of events for a given bin to be $\mathcal{N}_{i}$, the scaled 


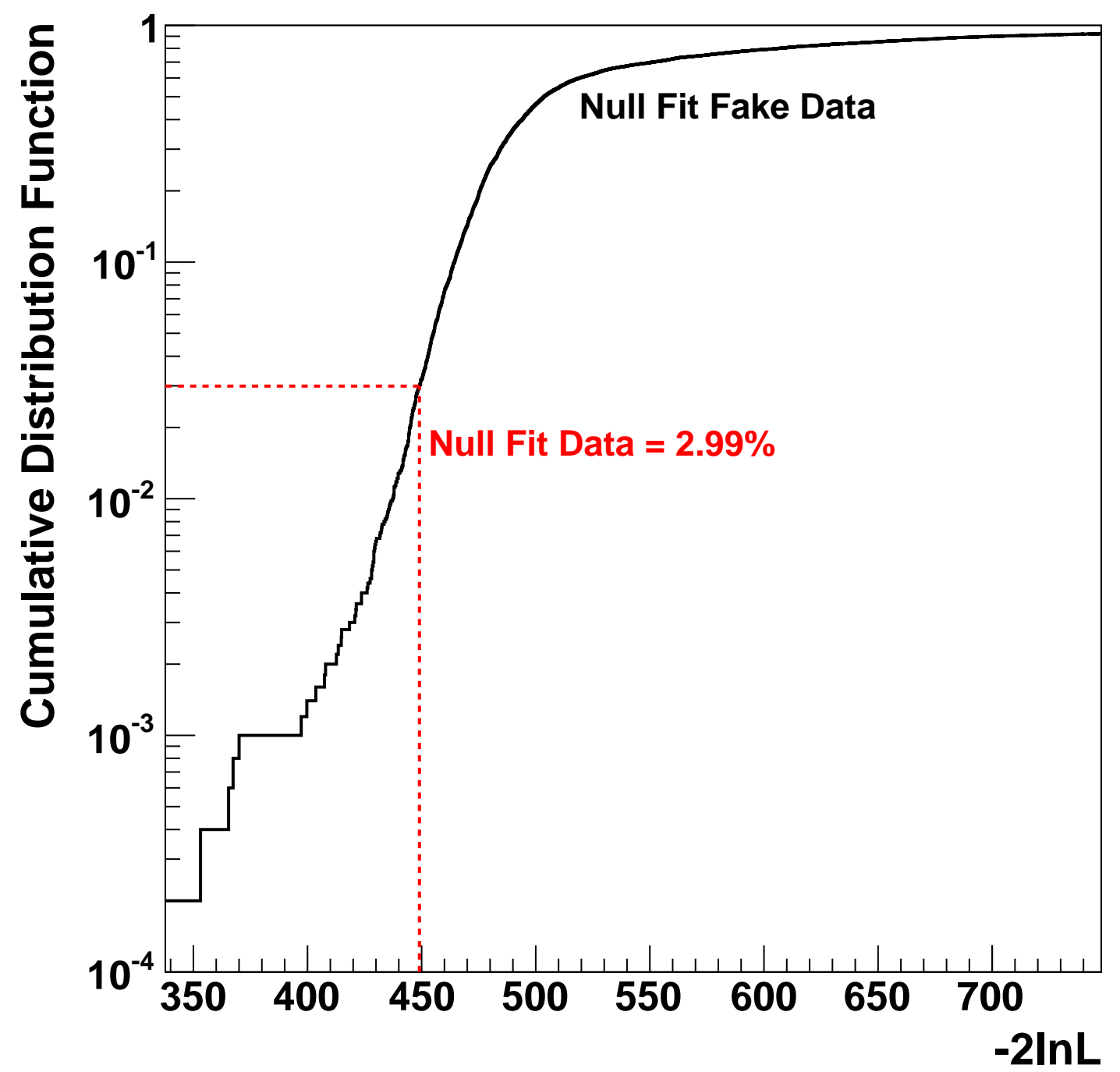

Figure 5.5: The cumulative probability distribution for signal best fit and null fit to null fake data sets. This shows that the probability that the prediction compared to data yields a test statistic this small (or smaller) is $3 \%$. 
number of events is given by

$$
N_{i}=\frac{\mathcal{N}_{i}}{f_{\mathrm{POT}_{i}}}
$$

where $f_{\mathrm{POT}_{i}}$ is the POT scaling in units of $1 \mathrm{e} 20 \mathrm{POT}$ for the data set corresponding to the $i$ th bin. For $\nu$-Mode (off-target) $f_{\mathrm{POT}_{i}}$ is equal to 6.461 (1.861). This means the statistical uncertainty for a given bin is then

$$
\sigma_{\text {stat }_{i}}=\frac{\sqrt{\mathcal{N}_{i}}}{f_{\text {POT }_{i}}}=\sqrt{\frac{N_{i}}{f_{\mathrm{POT}_{i}}}}
$$

and the covariance matrix scales as the following

$$
M_{i j}=\frac{M_{\mathcal{N}_{i j}}}{f_{\mathrm{POT}_{i}} f_{\mathrm{POT}_{j}}}
$$

where $M$ is in units of events/1e20 POT and $M_{\mathcal{N}}$ is in units of events. The constrained $\chi^{2}$ is given by

$$
\chi^{2}=\sum_{i, j}^{\text {all bins }}\left\{\left(N_{i}^{f i t}-N_{i}^{M C}\right) M_{i j}^{-1}\left(N_{j}^{f i t}-N_{j}^{M C}\right)+\frac{\left(N_{i}^{f i t}-N_{i}^{\text {data }}\right)^{2} f_{\mathrm{POT}_{i}} \delta_{i j} \delta_{i, \mathrm{con}}}{N_{i}^{\text {data }}}\right\},
$$

where $\delta_{i j}$ is the Kronecker delta, $N_{i}^{x}$ is the number of events/1e20 POT predicted in the $i$ th bin from source $x$, and $x$ can come from fit, MC or data. MC refers to the predicted background corrected by the background-only fit nuisance parameter results, and $M_{i j}$ is scaled so the fractional uncertainty stays the same. The fit distribution will be solved for assuming this $\chi^{2}$ distribution can be minimized. $\delta_{i, \text { con }}$ is given by

$$
\delta_{i, \mathrm{con}}=\left\{\begin{array}{cl}
1 & i \in \mathrm{CCQE}_{\nu} \\
1 & i \in \mathrm{NCE}_{\nu} \\
1 & i \in \mathrm{CCQE}_{\mathrm{Off}} \\
0 & i \in \mathrm{NCE}_{\mathrm{Off}}
\end{array}\right.
$$

and makes sures that only the non- $\mathrm{NCE}_{\mathrm{Off}}$ bins are being constrained by data.

To solve for $N_{i}^{f i t}$, the number of constrained events, the first derivative of Eq. (5.5) with respect to $N_{i}^{f i t}$ is set equal to zero. The steps are worked out in [89, 94, 95]. The 
final solution is given by

$$
N_{i}^{f i t}=\sum_{k} B_{i k} \sum_{j}\left\{M_{k j}^{-1} N_{j}^{M C}+f_{\mathrm{POT}_{k}} \delta_{k j} \delta_{k, \text { con }}\right\}
$$

where $\sigma_{m c, p r e d}$ stat $_{k}$ is the predicted statistical uncertainty given the MC prediction at the $k$ th bin, and

$$
B_{i j}^{-1}=\left\{\begin{array}{ll}
M_{i j}^{-1} & \delta_{i, \mathrm{con}} \text { or } \delta_{j, \mathrm{con}}=0 \\
M_{i j}^{-1}+\frac{f_{\mathrm{POT}_{i}} \delta_{i j}}{N_{i}^{\text {data }}} & \delta_{i, \mathrm{con}} \text { and } \delta_{j, \mathrm{con}}=1
\end{array} .\right.
$$

The test statistic $\chi_{\text {con }}^{2}$ is calculated to give how probable the constrain background-only fit. $\chi_{\text {con }}^{2}$ is given by

$$
\chi_{\text {con }}^{2}=\sum_{i, j}\left(N_{i}^{f i t}-N_{i}^{d a t a}\right) B_{i j}^{-1}\left(N_{j}^{f i t}-N_{j}^{d a t a}\right),
$$

and the constrained log-likelihood is

$$
-2 \mathcal{L}_{B}=\chi_{\text {con }}^{2}+\ln (|B|)
$$

Fig. 5.6 shows the probability for a null fit to data is consistent with MC predictions using Eq. (5.8) as the test statistic to be $8 \%$.

\subsubsection{PROPAGATING NUISANCE UNCERTAINTIES}

The resulting nuisance covariance matrix, shown in Fig. 5.7a, can be propagated to find the fit uncertainty for each bin.

Assume there is a function $w=g(u, v, \ldots)$; the difference between a given $w$ and the mean is

$$
w_{i}-\bar{w}=\left.\left(u_{i}-\bar{u}\right) \frac{\partial g}{\partial u}\right|_{x=w_{i}}+\left.\left(v_{i}-\bar{v}\right) \frac{\partial g}{\partial v}\right|_{x=w_{i}}+\cdots
$$

Using

$$
\sigma_{w_{i}}^{2}=\left(w_{i}-\bar{w}\right)^{2} \text { and } \sigma_{w_{i} w_{j}}=\left(w_{i}-\bar{w}\right)\left(w_{j}-\bar{w}\right)
$$

the variance on $w_{i} \sigma_{w_{i}}^{2}$ can be written as

$$
\sigma_{w_{i}}^{2}=\sigma_{u_{i}}^{2}\left(\left.\frac{\partial g}{\partial u}\right|_{x=w_{i}}\right)^{2}+\sigma_{v_{i}}^{2}\left(\left.\frac{\partial g}{\partial v}\right|_{x=w_{i}}\right)^{2}+\left.\left.2 \sigma_{u_{i} v_{i}} \frac{\partial g}{\partial u}\right|_{x=w_{i}} \frac{\partial g}{\partial v}\right|_{x=w_{i}}+\cdots
$$




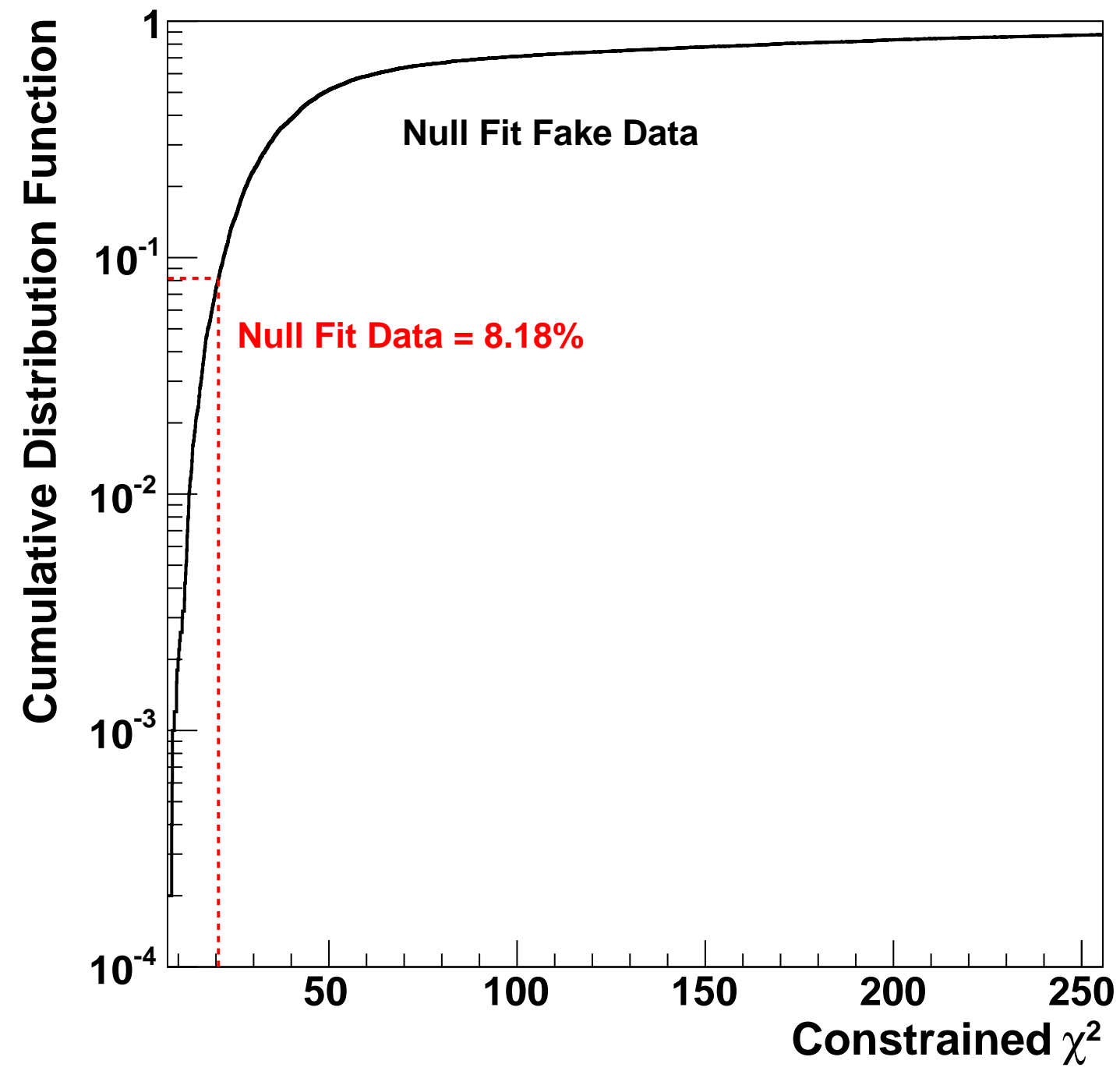

Figure 5.6: The cumulative distribution function distribution for the constrained fit test statistic when fitting null fake data with the null hypothesis, and where the background-only (null) fit to data falls in the cumulative distribution function distribution. The probability data is consistent with MC predictions using the constrained fit is $8 \%$. 


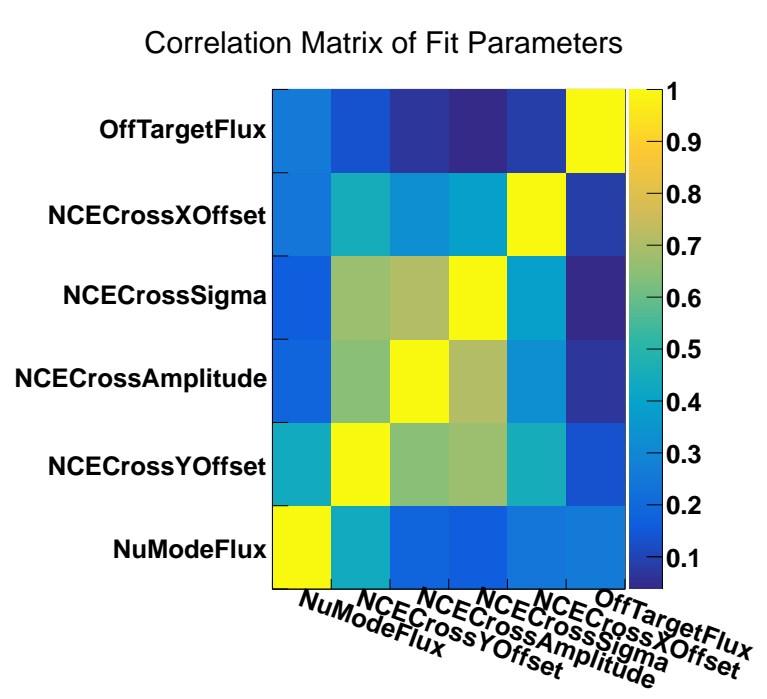

(a) Fit-Parameters

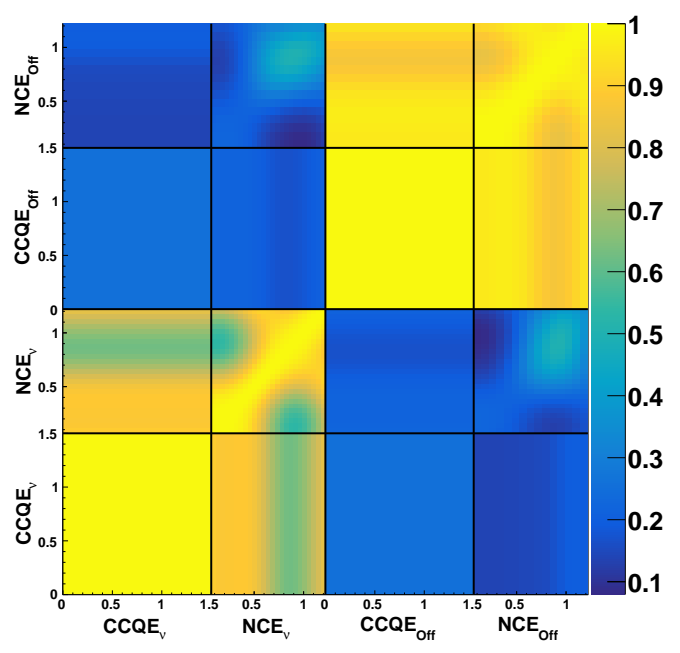

(b) Number of events as a function of $Q_{Q E}^{2}$

Figure 5.7: The correlation matrix for (a) fit parameters in background-only fit and (b) number of events after propagating the best fit parameters.

Extending Eq. (5.9) to include cross terms, the covariance $\sigma_{w_{i} w_{j}}$ is written as

$$
\begin{aligned}
\sigma_{w_{i} w_{j}}= & \left.\left.\sigma_{u_{i} u_{j}} \frac{\partial g}{\partial u}\right|_{x=w_{i}} \frac{\partial g}{\partial u}\right|_{x=w_{j}}+\left.\left.\sigma_{v_{i} v_{j}} \frac{\partial g}{\partial v}\right|_{x=w_{i}} \frac{\partial g}{\partial v}\right|_{x=w_{j}}+ \\
& \left.\left.\sigma_{u_{i} v_{j}} \frac{\partial g}{\partial u}\right|_{x=w_{i}} \frac{\partial g}{\partial v}\right|_{x=w_{j}}+\left.\left.\sigma_{v_{i} u_{j}} \frac{\partial g}{\partial v}\right|_{x=w_{i}} \frac{\partial g}{\partial u}\right|_{x=w_{j}}+\cdots
\end{aligned}
$$

Using Eq. (5.2) for the definition of $w=g(u, v, \ldots)$ and the results of the backgroundonly fit produces the propagated correlation matrix shown in Fig. 5.7b. Table 5.3 gives the propagated uncertainty for each data set with the assumption that the other three data sets are constraining that data set. Because $\mathrm{CCQE}_{\nu}, \mathrm{NCE}_{\nu}$ and $\mathrm{CCQE}_{\mathrm{Off}}$ are used to constrain $\mathrm{NCE}_{\mathrm{Off}}$, the chain fit reduces the total uncertainty on $\mathrm{NCE}_{\mathrm{Off}}$ from $33.5 \%$ to $12.8 \%$.

The low test statistic of the background-only fit suggests that the covariance matrix used is conservative. Regardless of the probability, fitting all four distributions at the same time decreases the effects of the systematics on the $\mathrm{NCE}_{\mathrm{Off}}$ sample by almost half. This suggests that sensitivity to a DM signal will be increased. The next step in fitting is to 


\begin{tabular}{lcccccc}
\hline \multirow{2}{*}{ Dist. } & \multicolumn{5}{c}{ Source total uncertainty $(\%)$} \\
& $\nu$ Flux & cross sections & det. model & total sys & stat & Fit Result \\
\hline $\mathrm{CCQE}_{\nu}$ & 5.9 & 16.6 & 3.0 & 17.9 & 0.3 & 12.2 \\
$\mathrm{NCE}_{\nu}$ & 5.5 & 13.2 & 13.5 & 19.9 & 0.3 & 10.8 \\
$\mathrm{CCQE}_{\text {Off }}$ & 50.7 & 18.2 & 2.8 & 54.0 & 3.2 & 23.4 \\
$\mathrm{NCE}_{\mathrm{Off}}$ & 31.4 & 7.9 & 8.2 & 33.5 & 2.6 & 12.8 \\
\hline
\end{tabular}

Table 5.3: The systematic and statistical uncertainty for each data set along with the propagated uncertainty looping only over the given data sets block.

assume a signal. A model independent fit was performed before testing the vector portal light dark matter model.

\subsection{MODEL INDEPENDENT FIT}

An efficiency-corrected excess true nucleon recoil energy $T_{\mathrm{N}}^{\text {true }}$ distribution was produced by adding fit parameters to the background-only fit. The excess has no assumption on shape, but requires the excess events come from a true NCE interaction and pass NCE selection cuts, and the neutrino nuclear and final state models in NUANCE are a good approximation. These assumptions are DM model independent and are termed model independent fit (MIF). The excess distribution is provided as a data release for theorists to use to test other DM models. This section describes developing the efficiency/smearing matrices and the fit results.

\subsubsection{CREATING EFFICIENCY MATRIX}

Fig. 5.8 shows the raw proton recoil energy $T_{p}^{\text {true }}$, defined as the difference in the incoming and outgoing neutrino energies, and the total efficiency as a function of $T_{N}^{\text {true }}$ for both protons and neutrons. The efficiency is about $35 \%$ above $T_{N}^{\text {true }} \geq 150 \mathrm{MeV}$ and drops to 


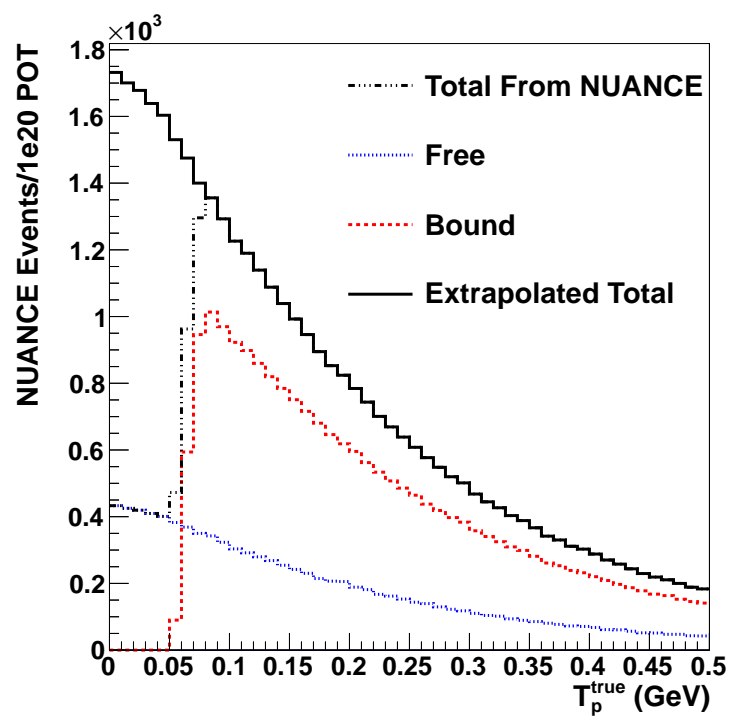

(a) $T_{p}^{\text {true }}$ from NUANCE

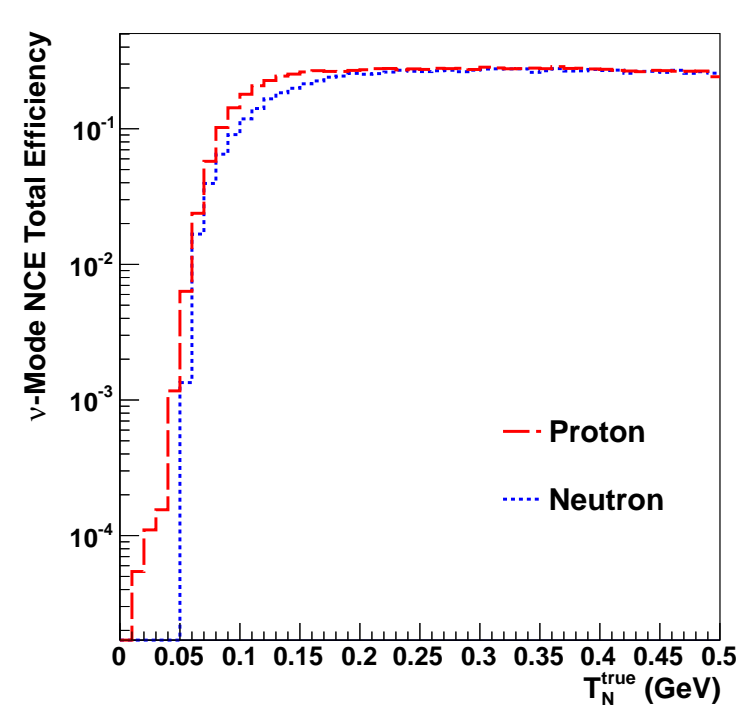

(b) NCE Total Efficiency

Figure 5.8: (a) $T_{p}^{\text {true }}$ coming from NUANCE, shown with the extrapolated distribution used to simulate every scattering is off a free nucleon. (b) Total efficiency of NCE events in the MiniBooNE detector. The total efficiency is the product of detector threshold, NCE selection criteria, and nuclear model so that results of MIF and confidence limit are corrected to free nucleon scattering. 
less than a percent around $50 \mathrm{MeV}$. The drop in the efficiency is due to detector efficiency, the requirement of needing 10 tank hits to generate a subevent, and the minimum $T_{N}^{\mathrm{reco}}$ cut (see Sec. 4.1.1). The efficiency also includes undoing of the nuclear model effects in carbon modeled by Pauli blocking at low $Q^{2}, T_{N}^{\text {true }} \lesssim 90 \mathrm{MeV}$. The Pauli blocking correction was treated as an efficiency so that results of the MIF are to be compared with scattering from free nucleons. The extrapolated proton distribution is used for the neutrons below the Pauli blocking threshold because there are no free neutrons in the detector. If the Pauli blocking correction was not made then the efficiency would be four times higher ( 8 total protons $/ 2$ free protons) below $90 \mathrm{MeV}$. This is a small adjustment to the dropping detector efficiency.

The new total shown in Fig. 5.9 is used as the true distribution to come up with the efficiency of MiniBooNE seeing a nuclear recoil in the detector with a given $T_{N}^{\text {true }}$ and applying NCE selection cuts (see Sec. 4.1.1). Fig. 5.9a shows the true distribution with fine

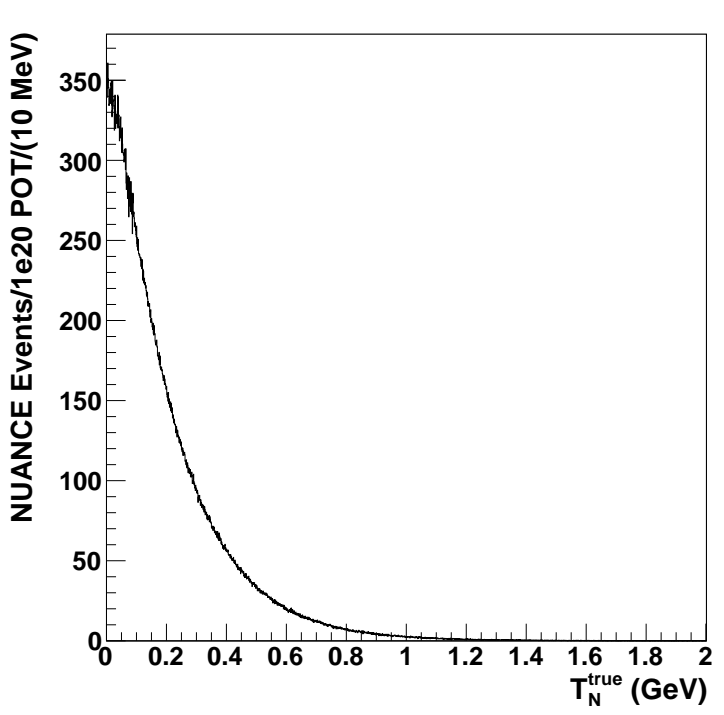

(a)

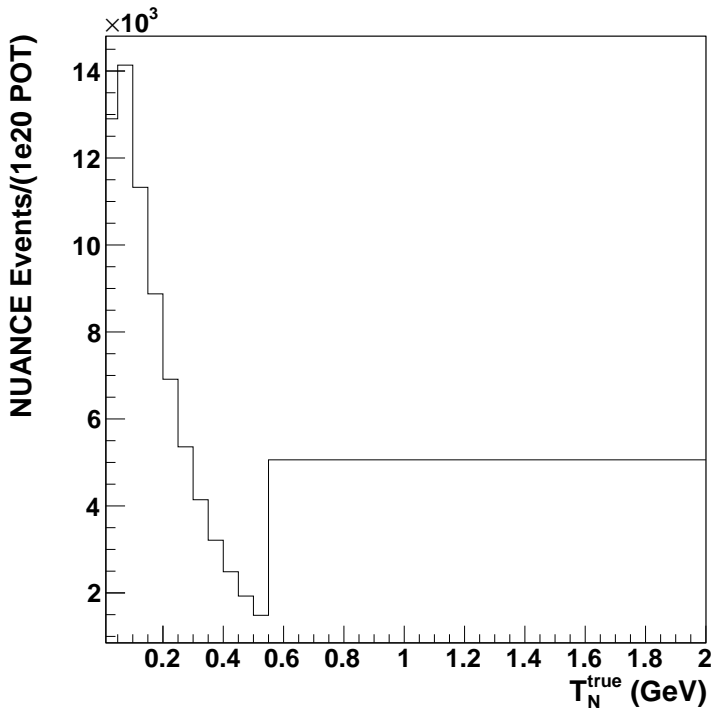

(b)

Figure 5.9: (a) The predicted $T_{N}^{\text {true }}$ coming from the MiniBooNE NUANCE simulation extrapolating to $0 \mathrm{GeV}$ as stated in Fig. 5.8a. (b) The same distribution but rebinned into the bins used in the excess search. 
binning while Fig. $5.9 \mathrm{~b}$ shows the true distribution with the binning used in the MIF.

The smearing matrices, to go from $Q_{Q E}^{2} \leftrightarrow T_{N}^{\text {true }}$, are shown in Fig. 5.10. The efficiency

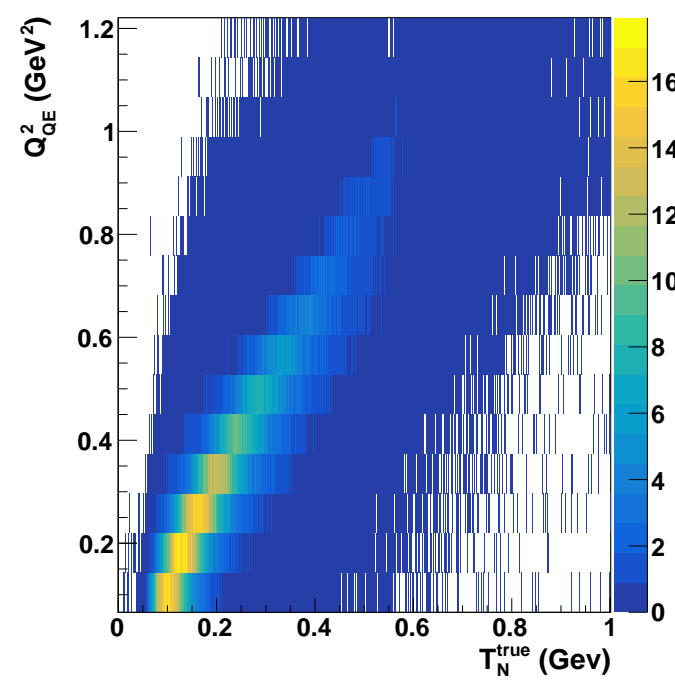

(a)

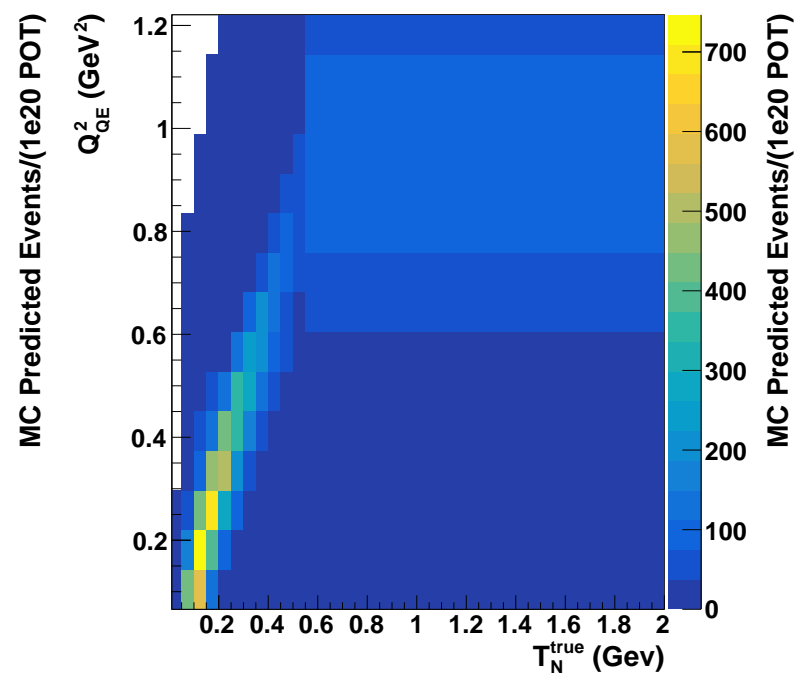

(b)

Figure 5.10: (a) The smearing matrix (to go from $Q_{Q E}^{2} \leftrightarrow T_{N}^{\text {true }}$ ) for NCE off-target $Q_{Q E}^{2}$ binning and the fine $T_{N}^{\text {true }}$ binning. (b) Same as (a) but with the coarse $T_{N}^{\text {true }}$.

for a given $T_{N}^{\text {true }}$ bin $x$ is given by

$$
\varepsilon_{x}=\frac{\sum_{y=1}^{n_{y}} S_{x y}}{N_{x}},
$$

where $n_{y}$ is the number of $Q_{Q E}^{2}$ bins, $S_{x y}$ is the $x, y$ bin in the smearing matrix, and $N_{x}$ is the number of true events in the $x$ bin. $S$ can then be normalized by

$$
S_{x y}^{\varepsilon}=\frac{S_{x y}}{\sum_{y^{\prime}=1}^{n_{y}} S_{x y^{\prime}}} .
$$

Fig. 5.11 shows $S^{\varepsilon}$ and $\varepsilon$ as a function of $T_{N}^{\text {true }}$. 


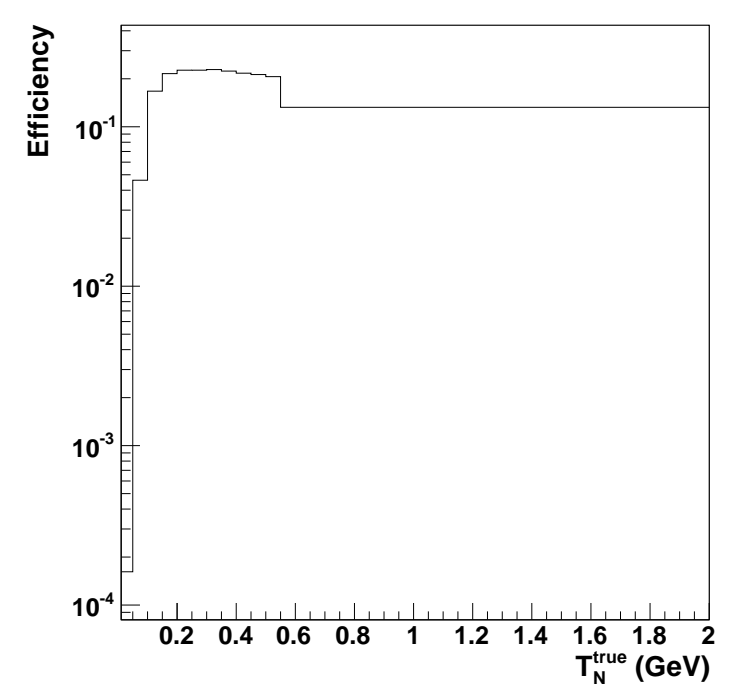

(a)

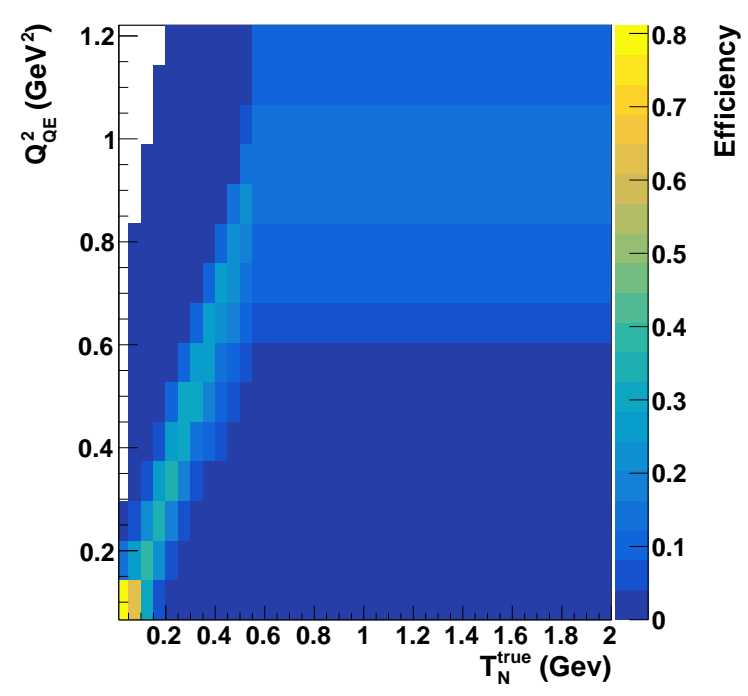

(b)

Figure 5.11: (a) Showing the $\varepsilon$ distribution given by Eq. (5.10), as well as (b) the $S^{\varepsilon}$ matrix shown with NCE off-target $Q_{Q E}^{2}$ binning.

\subsubsection{GENERATING PREDICTED $Q_{Q E}^{2}$ DISTRIBUTION}

Now that the $\varepsilon$ and $S^{\varepsilon}$ distributions are known the predicted reconstructed $Q_{Q E}^{2}$ distribution for a randomly given $T_{N}^{\text {true }}$ distribution is given by

$$
N_{y}^{\text {excess }}=\sum_{x=1}^{n_{x}} \varepsilon_{x} S_{x y}^{\varepsilon} N_{x}^{\text {excess }},
$$

were $N_{y}^{\text {excess }}\left(N_{x}^{\text {excess }}\right)$ is the predicted number of excess events for a given $Q_{Q E}^{2}\left(T_{N}^{\text {true }}\right)$ bin.

A fit of the possible number of events in the $T_{N}^{\text {true }}$ bins allowing only excess events in the $\mathrm{NCE}_{\mathrm{Off}}$ sample was performed using MINUIT. The resulting excess distribution is given in Fig. 5.12. The model independent fit yields $(130 \pm 270)$ excess events/(1e20 POT) integrated over $0.1<T_{N}^{\text {true }}<2 \mathrm{GeV}$.

When generating any efficiency a true radius has to be chosen. For the $\mathrm{CCQE}_{\nu}$ cross section analysis the true radius reported was $550 \mathrm{~cm}$ due to there being no events that pass cuts with a true radius greater than that number (see Fig. 5.13a). For the NCE analysis though true events go out to $610.6 \mathrm{~cm}$ (see Fig. 5.13b). The true radius used in calculating 


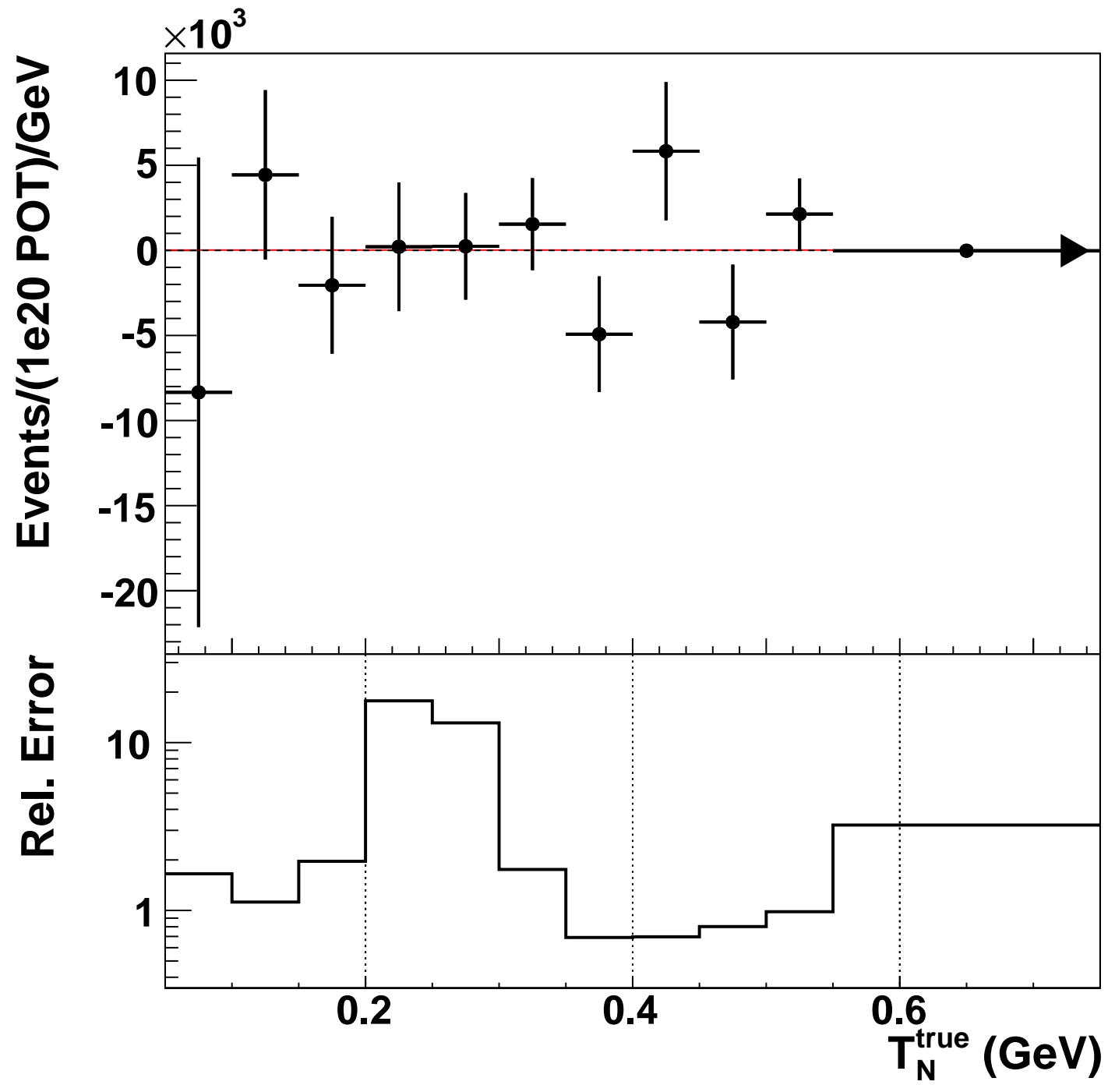

Figure 5.12: The results of the MIF as a function of $T_{N}^{\mathrm{true}}$. 


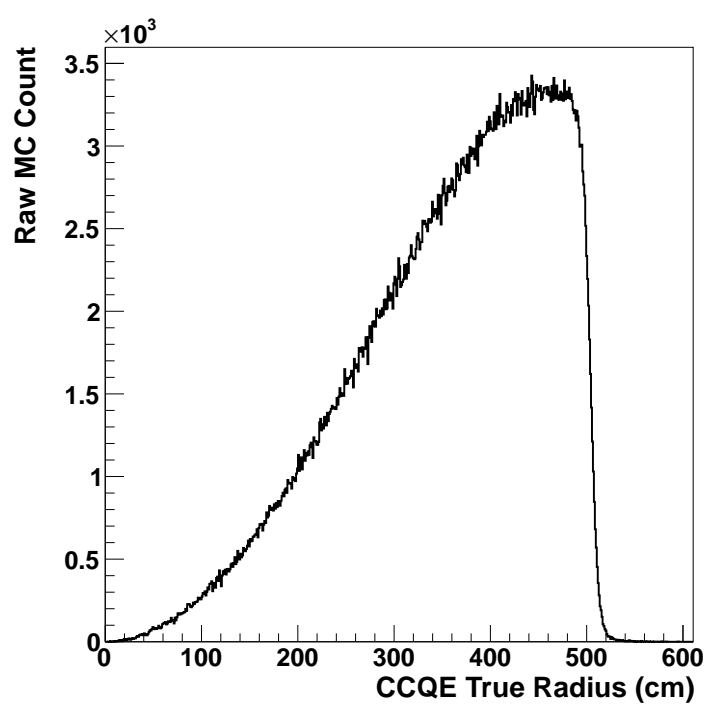

(a) charged-current quasielastic (CCQE)

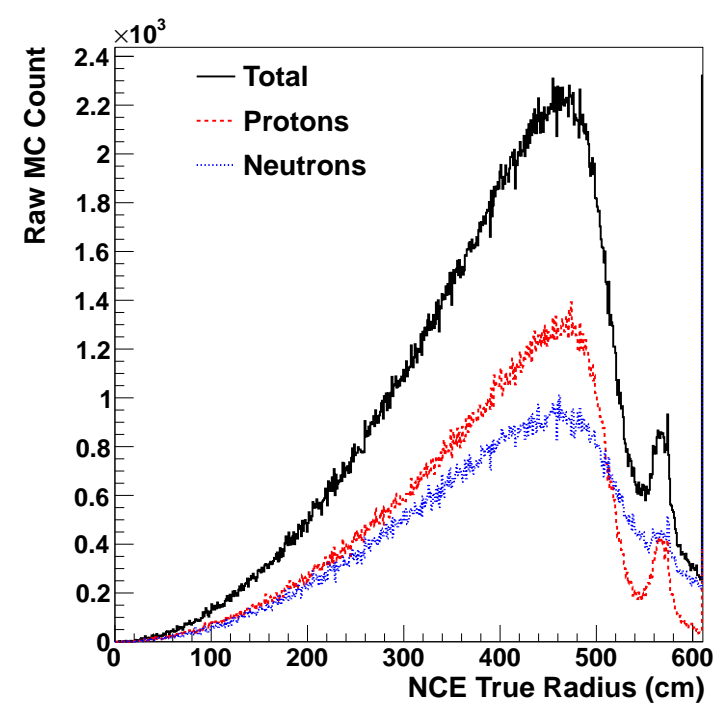

(b) NCE

Figure 5.13: The true radius position for (a) NUANCE $=1$ events passing CCQE cuts and (b) NUANCE $=2$ events passing NCE cuts.

the MIF was $610.6 \mathrm{~cm}$.

Fig. 5.14 shows the raw excess number of events/(1e20 POT) as a function of reconstructed $Q_{Q E}^{2}$. The black points show the result before applying nuisance parameters and gives an excess of -61 events/(1e20 POT). After applying initial guess nuisance parameters, the dotted line, the integrated excess is 7 events/(1e20 POT). The MIF result gives -7 events/(1e20 POT). The region of interest for sensitive light dark matter is to the left of the gray line. Although Table 4.9 shows there is a deficit in the $\mathrm{NCE}_{\mathrm{Off}}$ data, by applying the nuisance parameters an excess is seen, but the results from the MIF also suggests there is a deficit. Of course the deficit/excess are consistent with each other.

With the MIF and propagated fit results at hand, it is time to move on to setting confidence limits. 


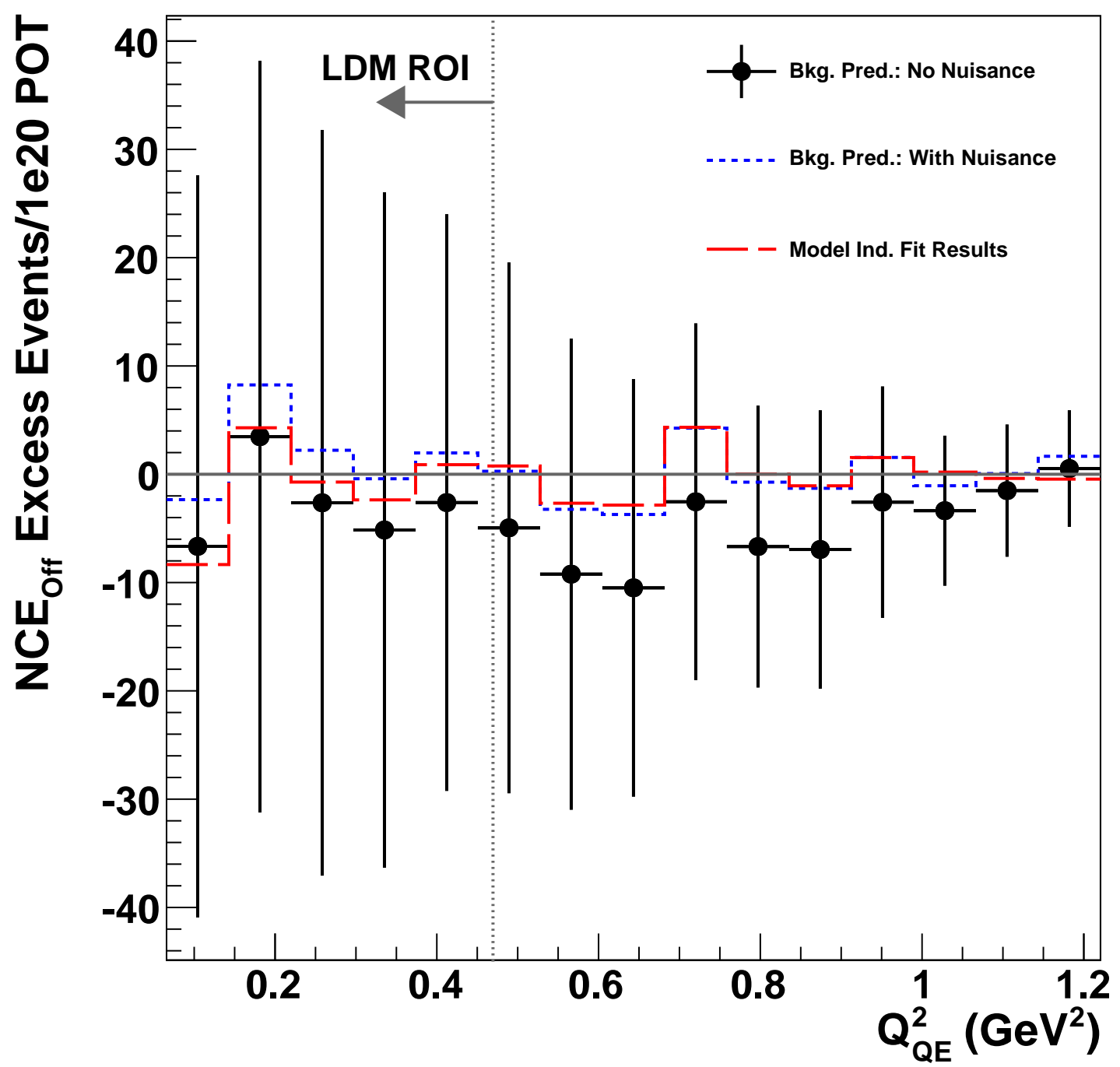

Figure 5.14: The excess number of events/(1e20 POT) as a function of reconstructed $Q_{Q E}^{2}$. The black points with error bars shows the data in Fig. 4.17a subtracted by the total prediction. The error bars on the black points is total uncertainty. The dotted line gives the results when adjusting the prediction by the initial guess nuisance parameters. The long dashed line gives the results of the MIF. The light dark matter (LDM) region of interest (ROI) is to the left of the vertical gray line. 


\section{CHAPTER 6}

\section{GENERATING CONFIDENCE LIMITS}

A confidence interval (CI) is the observed interval where an experiment knows, to a given confidence level, where the unobservable true value of the measurement lies based off the observable data at hand [96]. The confidence level is a predefined probability value that represents the probability of another experiment setup the exact same way will observe the same CI. A CI contains two sides and is therefore referred to as a bound limit. Each side of a CI is named a confidence limit (CL). If an experiment does not measure a significant amount of observable data over background then a lower or upper CL is reported.

To measure the MiniBooNE CL on light dark matter (LDM) using the nuclear recoil signature, a detailed simulation was developed to predict the LDM interaction in the MiniBooNE detector. The output was used as input for the MiniBooNE simulation (MC) to determine the predicted event distribution for LDM in MiniBooNE. Using these predictions in the fitting procedure (see Chapter 5) 90\% CL were produced. The CL were calculated using a frequentist technique. This chapter will explain the LDM simulation, and the CL analysis.

\subsection{LIGHT DARK MATTER SIMULATION}

A detailed customizable $\mathrm{C}++11$ simulation tool, BDNMC [28], was developed in paral-

lel with this analysis to simulate vector portal LDM production in the Booster Neutrino 
Beamline (BNB) in off-target mode (see Fig. 6.1). BDNMC does what BooNEG4BEAM,

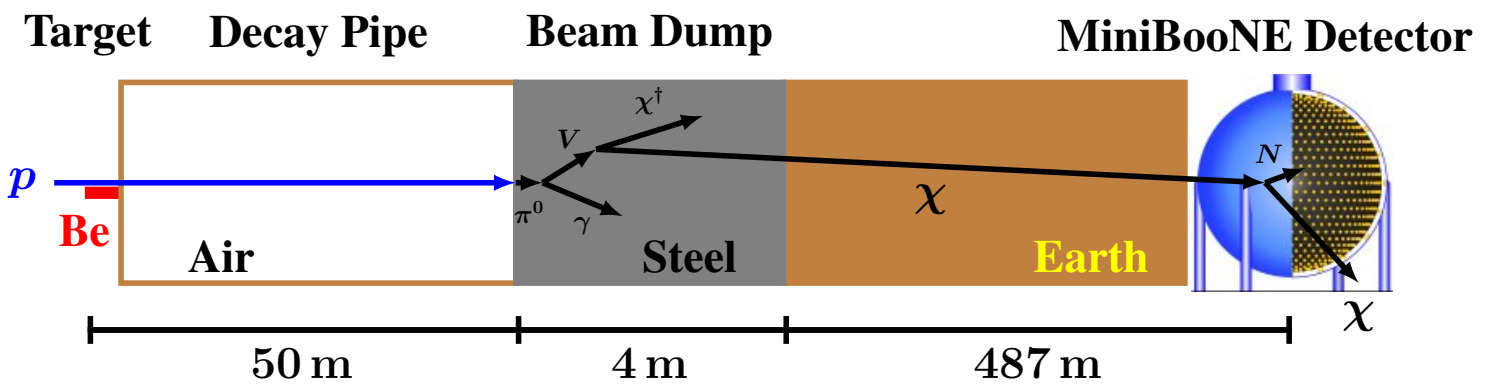

Figure 6.1: Schematic of vector portal LDM being produced in the off-target running configuration through $\pi^{0}$ decay.

BooBeamNT, and NuAnce (see Sections 3.1 and 3.2.3) do for neutrino production but for dark matter (DM) instead.

$\mathrm{BDNMC}$ is a generic simulation package that simulates the production of complex scalar LDM produced in a beam dump via various production channels and simulates the DM interacting in spherical or cylindrical detectors. Production channels used for this analysis are the $\pi^{0}, \eta$ on-shell decay and proton bremsstrahlung. The input meson energy vs angle distribution default for MiniBooNE is the Sanford-Wang distribution [62], though a particle list can be provided. The particle list is used when the Sanford-Wang distribution does not describe the meson production accurately. When using BDNMC, unless otherwise stated, the particle list was provided, because the average $\pi^{0}$ distribution simulated by BooNEG4BEAM is a composite of distributions of many materials, i.e. Sanford-Wang distribution for beryllium is not accurate enough (see Fig. 4.13a). The $\eta$ particle lists were generated by reweighting the $\pi^{0}$ distribution by mass.

BDNMC can simulate nucleon and electron elastic scattering as well as $\Delta$ production from inelastic scattering. For this analysis BDNMC was used to simulate nucleon scattering in the MiniBooNE detector (see Sec. 3.2). The number of DM particles produced from a 
given $\varphi$ decay is

$$
\begin{aligned}
N_{\chi, \varphi}=2 N_{V, \varphi} & =2 N_{\varphi} \operatorname{Br}\left(\varphi \rightarrow V+\gamma \rightarrow \chi \chi^{\dagger}+\gamma\right) \\
& =2 N_{\varphi} \operatorname{Br}(\varphi \rightarrow V+\gamma) \operatorname{Br}\left(V \rightarrow \chi \chi^{\dagger}\right) .
\end{aligned}
$$

For large values of $\alpha^{\prime}$, the branching ratio $\operatorname{Br}\left(V \rightarrow \chi \chi^{\dagger}\right) \simeq 1$ and

$$
\operatorname{Br}\left(\pi^{0} \rightarrow V+\gamma\right) \simeq 2 \epsilon^{2}\left(1-\frac{m_{V}^{2}}{m_{\pi^{0}}^{2}}\right)^{3}
$$

with $\operatorname{Br}(\eta \rightarrow V+\gamma)$ having a similar expression [24]. The number of $V$ produced through bremsstrahlung is

$$
N_{V}=\int_{0}^{p_{\perp}^{\max ^{2}}} d p_{\perp}^{2} \int_{z_{\min }}^{z_{\max }} d z \frac{d^{2} N_{V}}{d z d p_{\perp}^{2}} .
$$

This analysis uses BDNMC to simulate DM-nucleon scattering in the MiniBooNE detector. The simulated differential cross section, given in Eq. (2.7), includes the dipole term. The leading term is coupled to the electric charge of the nucleus, i.e. no coupling to neutrons, causing an asymmetry in the production of neutrons and protons. With the $\left(m_{V}^{2}-Q^{2}\right)^{-2}$ in the leading term the ratio of number of protons to number of neutrons produced is dependent on the $m_{V}$ and on the low- $Q^{2}$ cutoff, if one is applied while running the simulation. The low- $Q^{2}$ cutoff speeds up the simulation and only produces scatters that the detector would be sensitive to. Fig. 6.2 shows the ratio of protons to neutrons as a function of $m_{V}, m_{\chi}$ with a cut to not include any scatters that produced a nuclear recoil less than $20 \mathrm{MeV}$. The predicted number of events as a function of $m_{V}$ for a given $m_{\chi}, \epsilon$, and $\alpha^{\prime}$ combination is given in Fig. 6.3. The resonant peak around $780 \mathrm{MeV}$ comes from an increase in the production cross section of $V$ because of contributions from $\rho \rightarrow V$ mixing. Below $\mathrm{m}_{\pi^{0}}, V$ is predominately produced through decay of $\pi^{0} \mathrm{~s}$.

BDNMC produces an event list giving the 4-momentum of the parent meson, if through meson decay channel, the 4-momentum of $V$ and $\chi$, and the 4-momentum and 4-position vectors for the scattered particle in the detector. BDNMC was run under the assumption that the scattered particle is a free nucleon. 


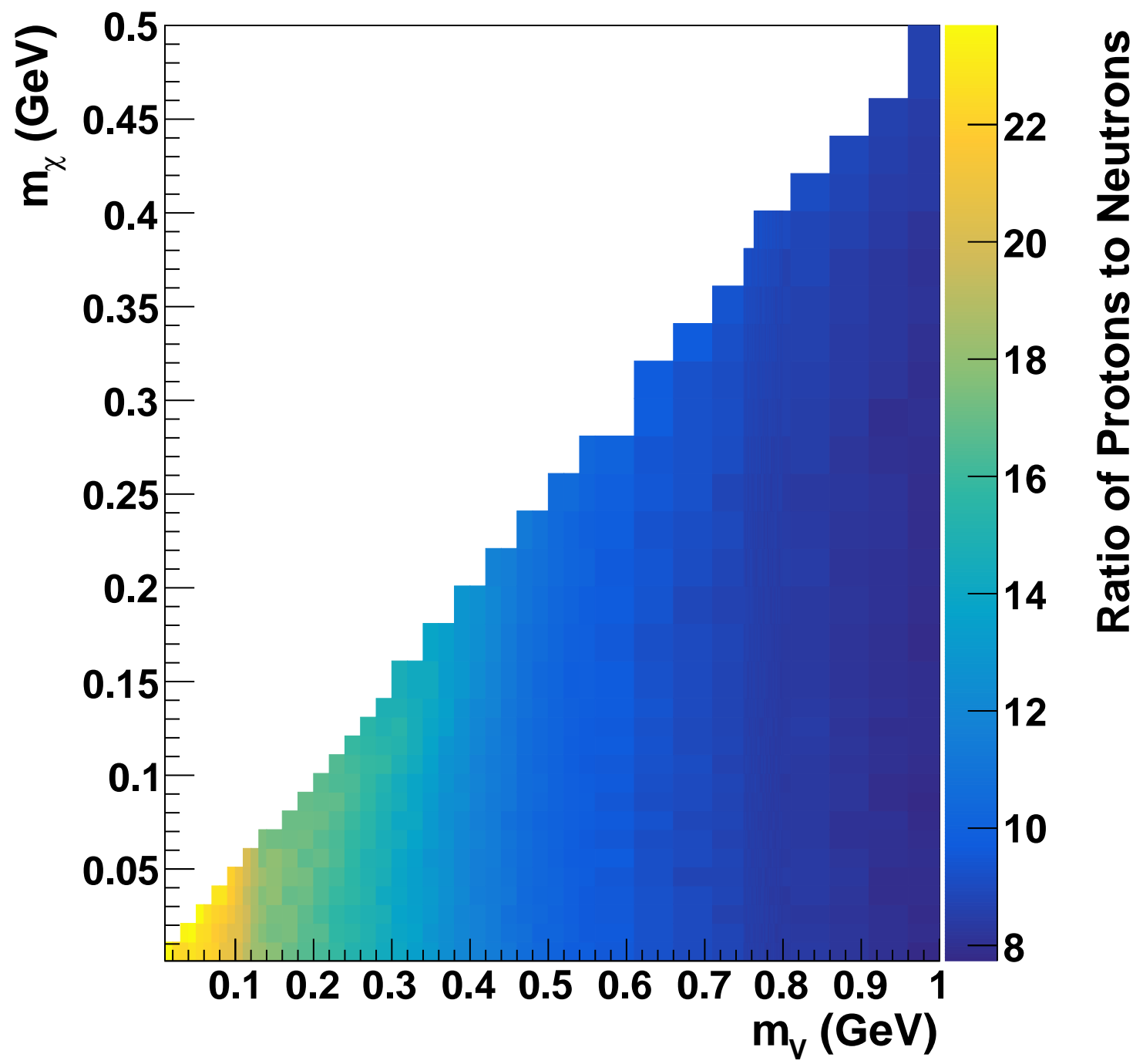

Figure 6.2: Ratio of number of protons to number of neutrons produced in DM-nucleon scattering with a low nuclear recoil cut off at $20 \mathrm{MeV} . \epsilon=10^{-3}$ and $\alpha^{\prime}=0.1$. 


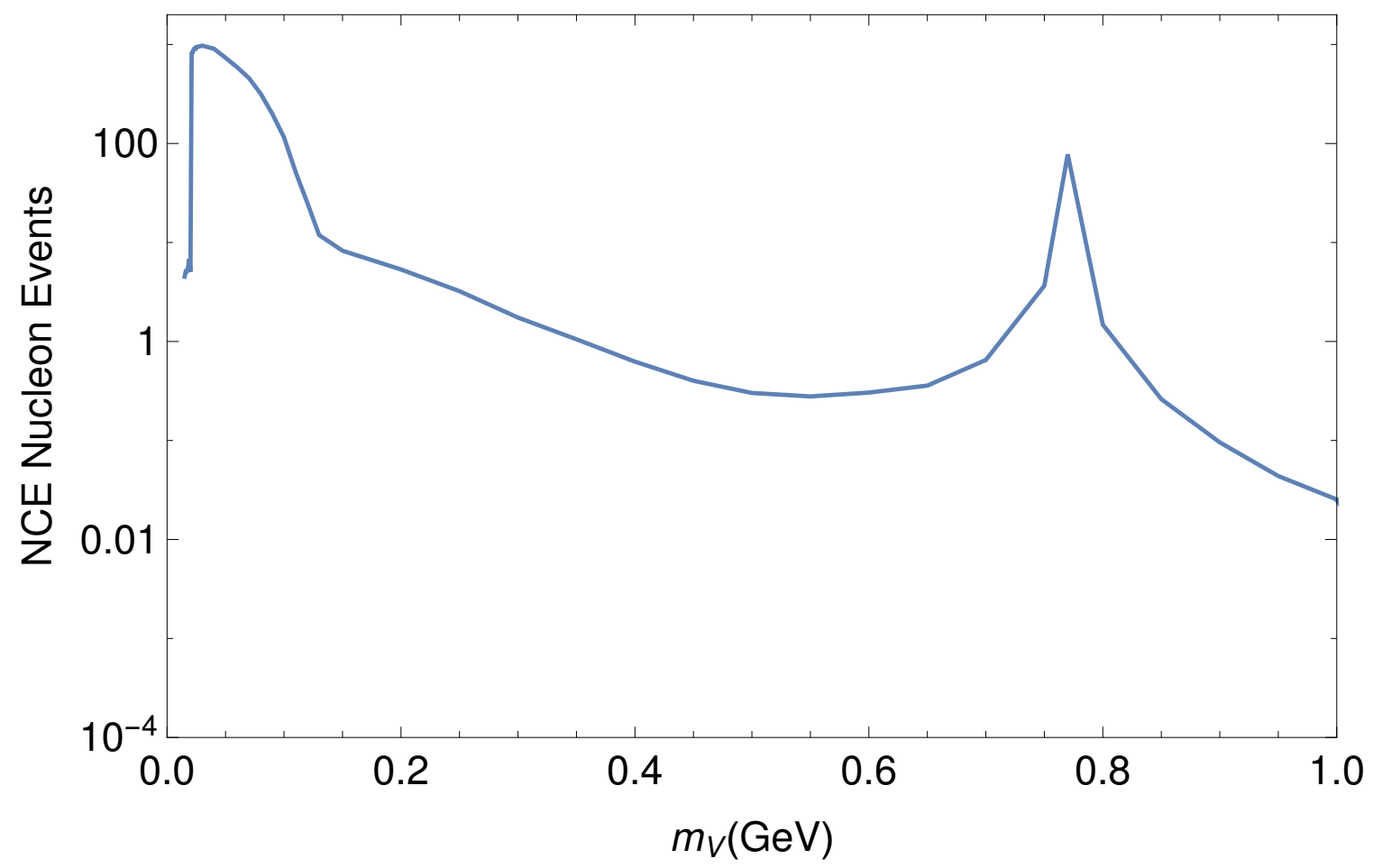

Figure 6.3: Predicted number of nucleons from DM scattering in MiniBooNE, assuming $2 \times 10^{20} \mathrm{POT}$, as a function of $m_{V}$ with $m_{\chi}=10 \mathrm{MeV}, \epsilon=10^{-3}$, and $\alpha^{\prime}=0.1$. Copied from $[28]$. 
The predicted number of events/(1e20 protons on target (POT)) and the average of the true nuclear recoil energy $\left\langle T_{N}^{\text {true }}\right\rangle$ are given in Fig. 6.4. As $m_{V}$ increases $\left\langle T_{N}^{\text {true }}\right\rangle$ also increases

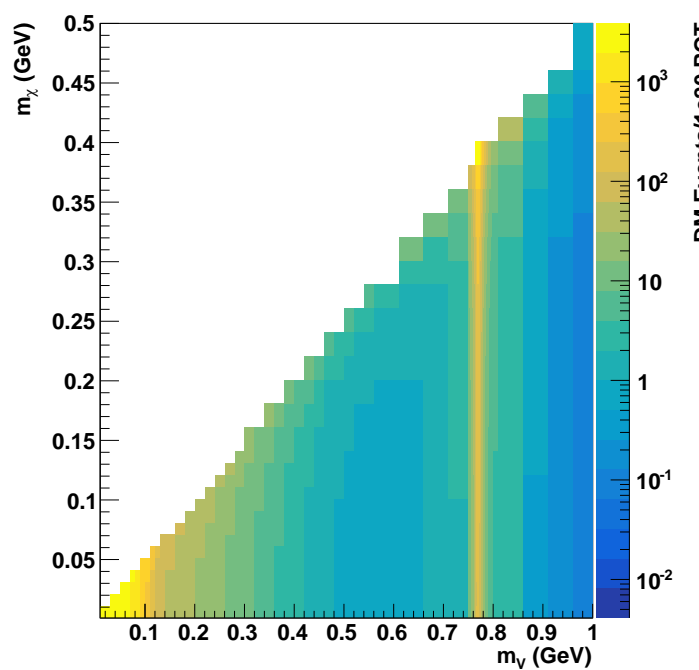

(a) Integral

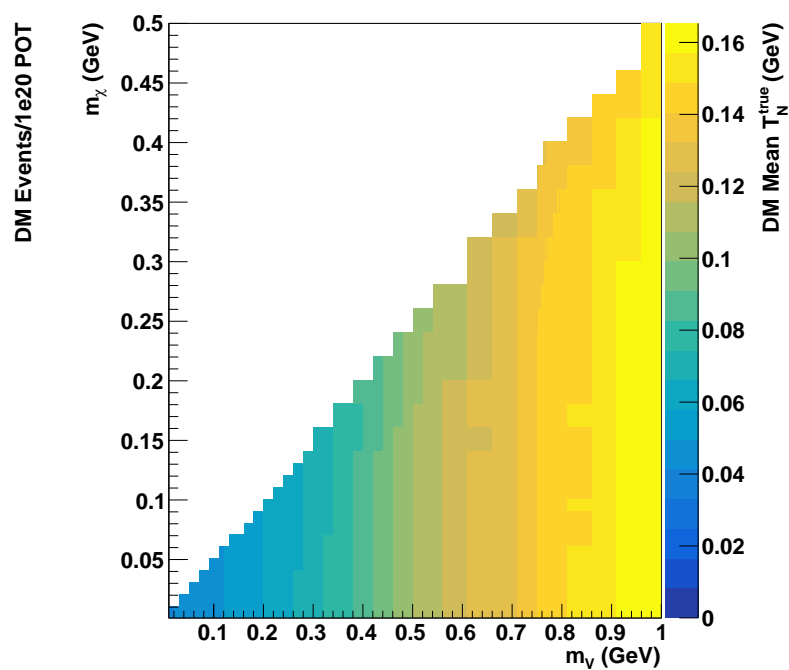

(b) Mean True Recoil Energy

Figure 6.4: The predicted number of events/(1e20 POT $)$ and mean true nucleon recoil energy coming out of BDNMC. Both plots use an efficiency equal to 1 as well as $\epsilon=$ $10^{-3}$ and $\alpha^{\prime}=0.1$. The lighter $m_{V}, m_{\chi}$ combination the softer the recoil nucleon energy spectrum.

while the number of predicted raw events decreases, except for around the $\rho$ mass. There is not much dependence on $m_{\chi}$, though if $m_{\chi}$ equals half of $m_{V}$ there is a discontinuity which causes the probability of decay to increase.

Example $T_{N}^{\text {true }}$ distributions are shown in Fig. 6.5a with an artificially low energy cut off below MiniBooNE detection. The two LDM parameters were chosen to show the sensitivity comparing low and high $m_{V}$ values. Fig. 6.5b applies the ratio of BDNMC prediction to NUANCE neutral-current elastic (NCE) truth information. Protons and neutrons are handled separately because of the asymmetry in production. True neutrino NCE interactions are used regardless of final state interactions to correctly handle all efficiencies correctly.

With the total efficiency in MiniBooNE dropping below $~ 90 \mathrm{MeV}$, MiniBooNE is most 


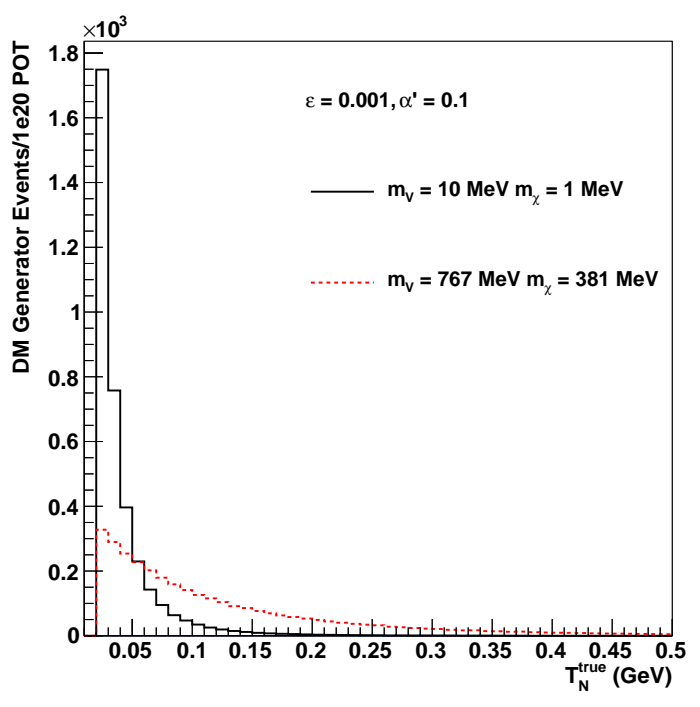

(a) Efficiency $=1$

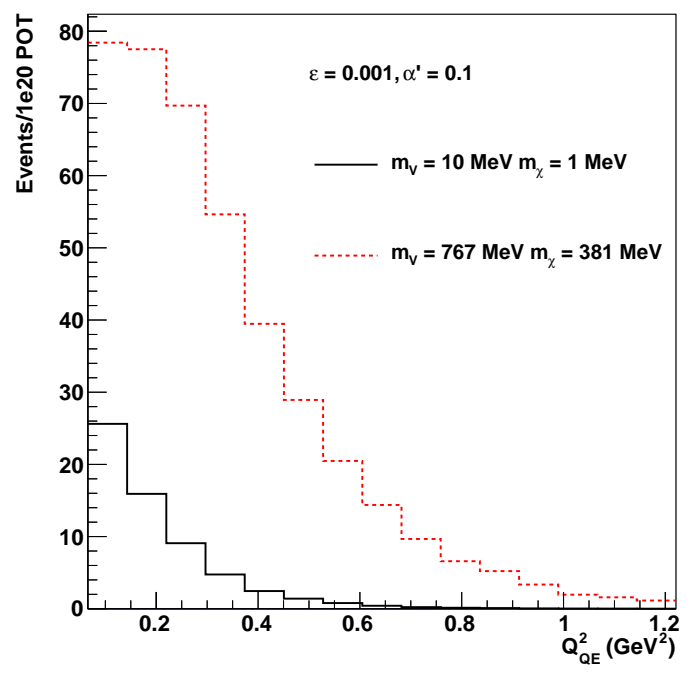

(b) Efficiency Applied

Figure 6.5: (a) Produced $T_{N}^{\text {true }}$ distribution from BDNMC for different combinations of $m_{V}, m_{\chi}$. (b) Reweighted DM prediction as a function of $Q_{Q E}^{2}$ for the same combinations of $m_{V}, m_{\chi}$ used in (a). Both plots have $\epsilon=10^{-3}$ and $\alpha^{\prime}=0.1$.

sensitive to LDM when the average efficiency applied nuclear recoil energy is greater than or equal to $100 \mathrm{MeV}$. The difference in the predicted reconstructed distribution for two combinations of $m_{V}, m_{\chi}$ is shown Fig. 6.5b. The weight of a simulated event is given by Fig. 6.5a divided by Fig. 5.9a. Because BDNMC does not contain a nuclear model, the extrapolated NUANCE result was used so the weight for the event is based on free nucleon interactions only.

In summary, for this analysis the following conditions were used when running BDNMC: 1) only included on-shell decays, $\left.2 m_{\chi}<m_{V}, 2\right)$ mixing angle between dark and light sectors: $\epsilon=0.001$ for all runs, 3) coupling strength between the vector mediator and the particle: $\alpha^{\prime}=0.1$ for all runs, 4) production channels $=\pi^{0}$-decay, $\eta$-decay, bremsstrahlung, 5) included event list of $\pi^{0}$ and $\eta$ generated from the MiniBooNE BooNEG4BEAm simulation for off-target running, and 6) outgoing nucleon kinetic energy restricted to be between 0.02 to $8.9 \mathrm{GeV}$. 


\subsection{CONFIDENCE LIMITS}

For each combination of $m_{V}, m_{\chi}$ BDNMC generated $2 \times 10^{5}$ simulated nucleon scatters in the detector. These events were then scaled by the probability of 1 scatter to occur per 1e20 POT. Each combination of $m_{V}, m_{\chi}$ is treated independently of all other combinations, where the CL is set on the combination $\epsilon^{4} \alpha^{\prime}$. Two different CL methods were considered, the "traditional" $\chi^{2}$ and a frequentist method. The frequentist method follows the MiniBooNE oscillation analysis [89, 93], which is based off the method developed by Feldman and Cousins [97].

With the addition of the DM scale parameter $\epsilon^{4} \alpha^{\prime}$, finding the minimum of the six nuisance parameters (see Sec. 5.2) plus $\epsilon^{4} \alpha^{\prime}$ is dependent on the initial parameter values because the minimum of the parameter space is so wide MINUIT thinks it found the minimum. But if one of the parameters is fixed then a "better minimum", i.e. lower test statistic, could be found. To avoid this, the nuisance parameters were fixed to their initial guesses (see Sec. 5.2) to make sure the fits during the CL procedure behave.

An important difference between this fit and those described in Chapter 5 is the covariance matrix was held fixed. This ensures that if the fake data generated bins with zero content the covariance matrix will still be invertible. The size of the systematic component was based on the original background prediction and the statistical component was determined by the data or fake data generated.

\subsubsection{TRADITIONAL $\chi^{2}$ METHOD}

The traditional $\chi^{2}$ approach to setting confidence limits is applicable when the test statistic is of quadratic form. The CL is deduced from the intersection of

$$
\Delta \chi_{i}^{2}=\chi_{i}^{2}-\chi_{\min }^{2}
$$


with a probability determined from the $\chi^{2}$ probability distribution function (PDF) where the number of degrees of freedom is equal to the number of independent parameters [98]. This method requires no fake data studies and is sometimes referred to as the "graphical method" [98]. Define $\Delta \chi_{c}^{2}$ as the value at which $90 \%$ of Eq. (6.1) is less than itself.

For this analysis the log-likelihood test statistic was used instead of the $\chi^{2}$ test statistic. Wilks' Theorem [99] is traditionally used to approximate the log-likelihood ratio

$$
\Delta(-2 \ln L)=(-2 \ln L)_{i}-(-2 \ln L)_{\min }
$$

as a $\chi^{2}$ distribution. It is valid in large data samples. For this analysis where each combination of $m_{V}, m_{\chi}$ are treated independently, Eq. (6.2) can be rewritten as

$$
\begin{aligned}
& \Delta(-2 \ln L)_{\epsilon^{4} \alpha^{\prime}}=(-2 \ln L)_{m_{V}, m_{\chi}, \epsilon^{4} \alpha^{\prime}}-(-2 \ln L)_{m_{V}, m_{\chi}, \min } \\
\Rightarrow & \Delta(-2 \ln L)_{\mathrm{Sig}}=L_{\mathrm{Sig}}-L_{\mathrm{BF}},
\end{aligned}
$$

since the minimum test statistic should equate to the best fit parameter combinations. $L_{\mathrm{Sig}}$ fixes $\epsilon^{4} \alpha^{\prime}$ to a specified value while $L_{\mathrm{BF}}$ lets $\epsilon^{4} \alpha^{\prime}$ float in the fit. The special case when $\epsilon^{4} \alpha^{\prime}=0$ will be labeled as $L_{\text {Null }}$ since the fit does not contain any DM. Fig. 6.6 gives the $L_{\mathrm{BF}}$ and $-\left(\epsilon^{4} \alpha^{\prime}\right)_{\mathrm{BF}}$ for each combination of $m_{V}, m_{\chi}$ that were tested when fitted to data. $\left(\epsilon^{4} \alpha^{\prime}\right)_{\mathrm{BF}}$ is the resulting DM scale parameter that corresponds to $L_{\mathrm{BF}}$. Over the entire LDM mass parameter space there is little variation in the test statistic, but there is a large range in $\left(\epsilon^{4} \alpha^{\prime}\right)_{\mathrm{BF}}$. The large variation in $\left(\epsilon^{4} \alpha^{\prime}\right)_{\mathrm{BF}}$ is consistent with Fig. 6.4, and the uncertainty on $\left(\epsilon^{4} \alpha^{\prime}\right)_{\mathrm{BF}}$ is always greater than $100 \%$. The small change in $L_{\mathrm{BF}}$ suggests that one $m_{V}, m_{\chi}$ combination is not more likely to describe the difference between data and background prediction.

Using Wilks' theorem, the $90 \% \mathrm{CL}$ is set to the intersection of $\Delta(-2 \ln L)$ with the value of 2.67. If there are two intersections in physical parameter space, i.e. $\epsilon^{4} \alpha^{\prime} \geq 0$, then a bounded limit is reported. If only one intersection is found then a one sided limit is reported. Fig. 6.7 illustrates this method with fits to data as black dots and the line equal 


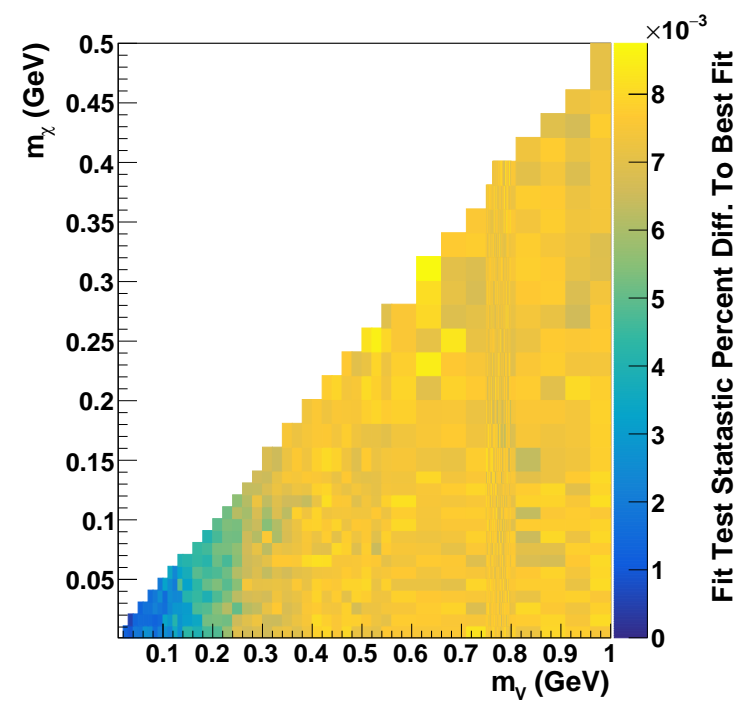

(a) $L_{\mathrm{BF}}$

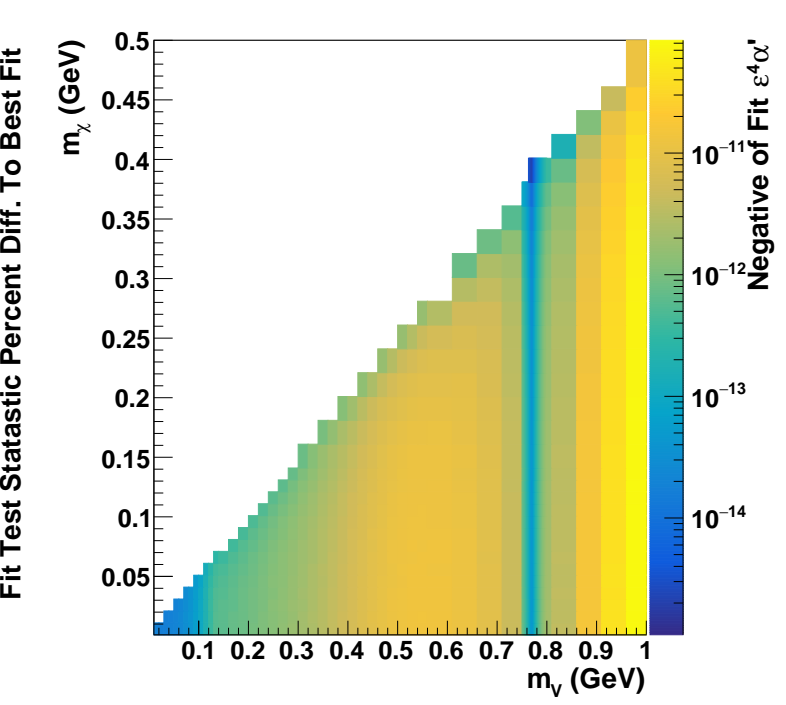

(b) $-\left(\epsilon^{4} \alpha^{\prime}\right)_{\mathrm{BF}}$

Figure 6.6: The percent difference (a) corresponding $-\left(\epsilon^{4} \alpha^{\prime}\right)$ (b) as a function of $m_{V}, m_{\chi}$ are the results of the best fit to data.

to 2.67 given as an orange solid horizontal line. The other lines in Fig. 6.7 are discussed in Sec. 6.2.2.

\subsubsection{FREQUENTIST METHOD}

The only assumption in the frequentist method for setting CLs is the test statistic used, e.g. $\Delta \chi^{2}$ or ratio of log-likelihoods. The ratio of log-likelihoods is more general and is what is used here. A fully frequentist approach uses fake data to generate both sensitivity and CL curves. The sensitivity curve is the predicted CL for the experiment based on background studies only. It shows how much signal has to be added to the background studies before a change is seen at a given confidence level. No data is used in constructing the experiment's sensitivity. Confidence limits are generated using fits to data and to the same fake data used to generate the sensitivity curve.

The fake data were generated using Cholesky decomposition described in Sec. 5.1. Initial parameters were calculated for each fake data generated, so no nuisance parameter 


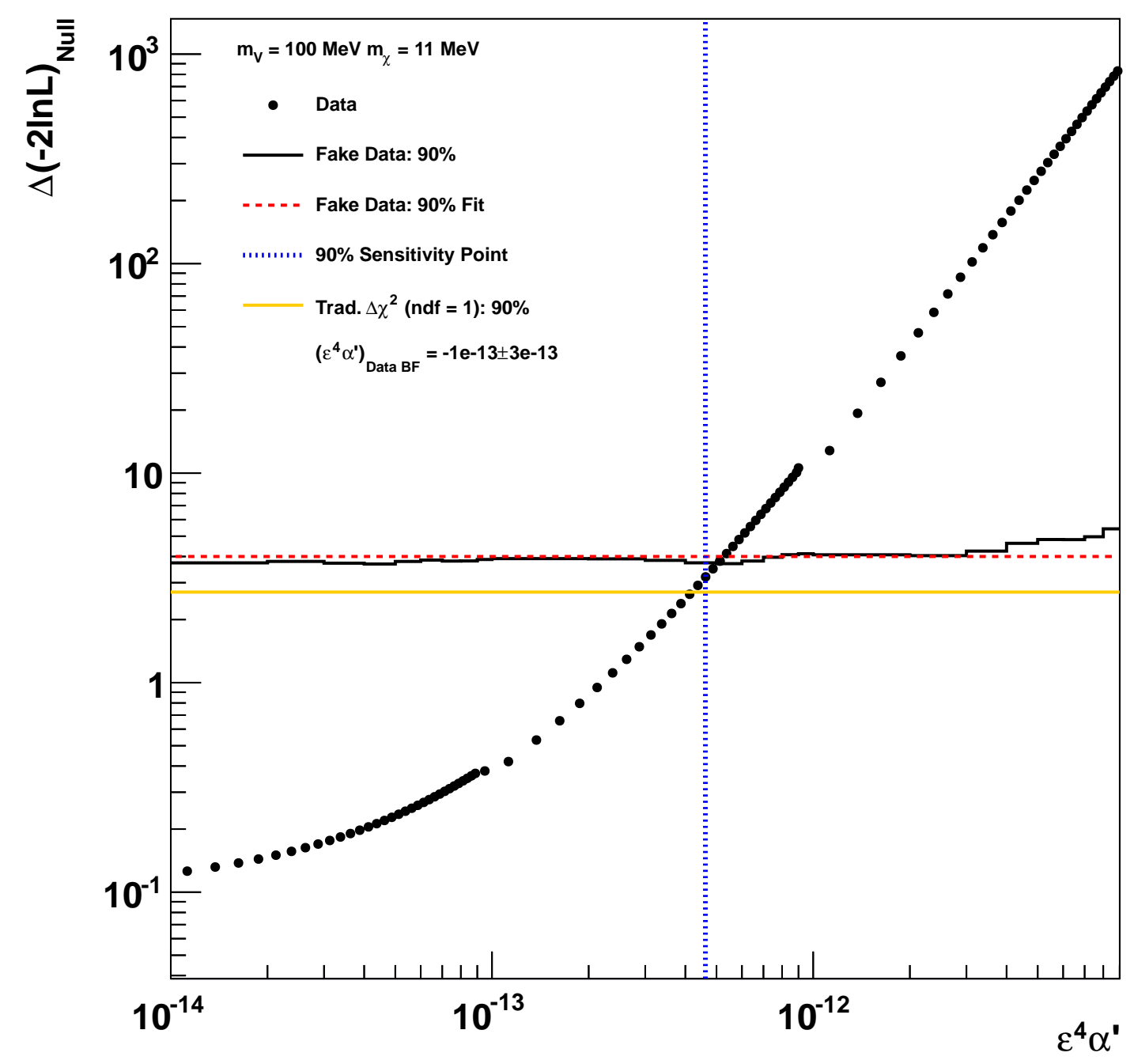

Figure 6.7: An illustration of the CL procedure for a given $m_{V}, m_{\chi}$ combination. The data distribution is given by black points, the $90 \%$ values from frequentist fake data is the black line, a linear fit to the fake data is the red dashed line, the traditional $\chi^{2}$ value used is given by the solid orange line, and the predicted sensitivity using SensMethod1 is shown as a vertical blue dotted line. 
corrections were made to the background prediction before generating the fake data. This ensured the sensitivity was not biased by off-target data.

\section{GENERATING SENSITIVITY}

To generate the sensitivity, 5000 null fake data sets were generated. Null fake data sets are fake data sets where no DM is added to the background prediction before generating the fake data. In contrast, signal fake data sets are added to the background prediction by the predicted amount of signal before generating the fake data. To reduce any bias in the fitting method the difference $\Delta(-2 \ln L)_{\text {Null }}$ is calculated where

$$
\Delta(-2 \ln L)_{\mathrm{Null}}=L_{\mathrm{Null}}-L_{\mathrm{BF}}
$$

Fig. 6.8 gives $\Delta(-2 \ln L)_{\text {Null }}$ compared to different $\chi^{2}$ distributions with different degrees of freedom and different assumptions on $\epsilon^{4} \alpha^{\prime}$ with $m_{V}=100 \mathrm{MeV}, m_{\chi}=11 \mathrm{MeV}$. Calculating $\Delta \chi_{c}^{2}$ for the $\Delta(-2 \ln L)_{\text {Null }}$ distribution (vertical red line in Fig. 6.8a) shows that if Wilks' theorem was assumed (vertical orange dotted line in Fig. 6.8a), the resulting CL will be stronger than the fake data suggests. The effective degrees of freedom for the DM signal is greater than 1 . This alone suggests the traditional $\chi^{2}$ method is not valid in this case.

2000 signal fake data sets were generated for each $\epsilon^{4} \alpha^{\prime}$. For each signal fake data $\Delta(-2 \ln L)_{\text {Null }}$ is calculated and the PDF are shown in Fig. 6.8b. By increasing $\epsilon^{4} \alpha^{\prime}$ there is a point when $\Delta(-2 \ln L)_{\text {Null }}>\Delta \chi_{c}^{2}$ for all fake data sets, but since $\epsilon^{4} \alpha^{\prime} \geq 0$ there will never be a point when $\Delta(-2 \ln L)_{\text {Null }}<\Delta \chi_{c}^{2}$ for all generated fake data sets.

To generate the sensitivity, two methods, labeled SensMethod1 and SensMethod2 were compared with each other.

SensMethod1: This method, used in the oscillation analysis [93], calculates the mean of

$$
\Delta(-2 \ln L)_{\text {Null }}\left\langle\Delta(-2 \ln L)_{\text {Null }}\right\rangle \text { for each } \epsilon^{4} \alpha^{\prime} \text { simulated. The sensitivity for the } m_{V}, m_{\chi}
$$




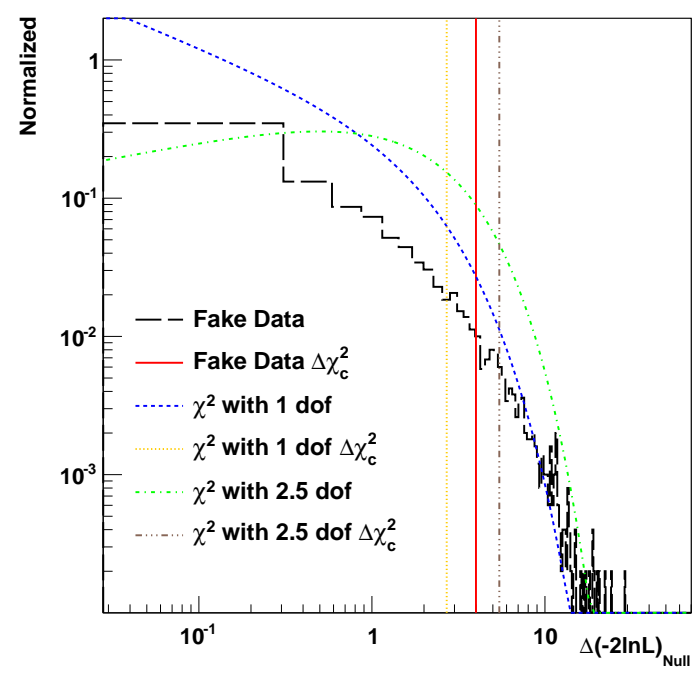

(a) Compare with $\chi^{2}$ assumption

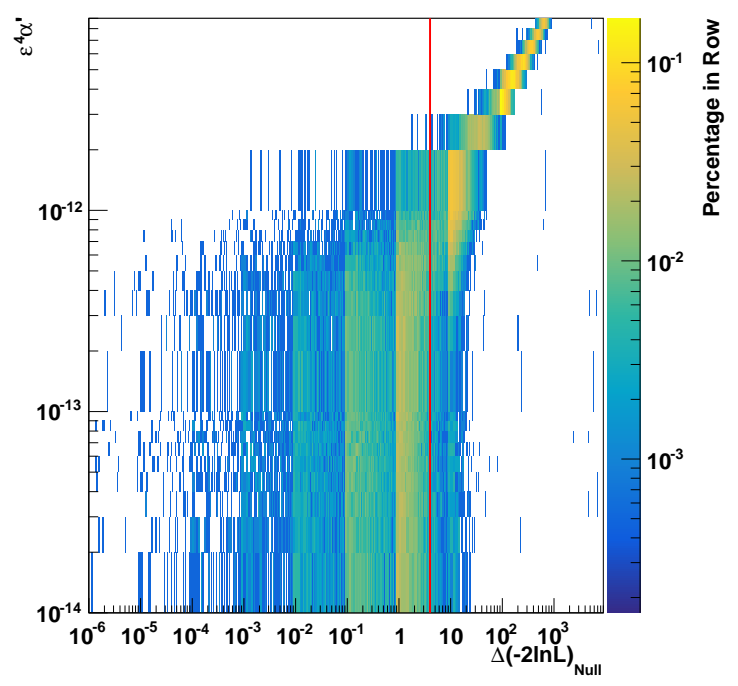

(b) Comparing different $\epsilon^{4} \alpha^{\prime}$

Figure 6.8: (a) The null fake data $\Delta(-2 \ln L)_{\text {Null }}$ distribution compared to $\chi^{2}$ distributions with different number degrees of freedom, and (b) the probability distribution function of $\Delta(-2 \ln L)_{\text {Null }}$ for different $\epsilon^{4} \alpha^{\prime}$ fake data. The vertical red line in (b) is the same as the vertical red line in (a). 
combination is the value of $\left(\epsilon^{4} \alpha^{\prime}\right)_{\text {Sens }}$ that produces $\left\langle\Delta(-2 \ln L)_{\text {Null }}\right\rangle=\Delta \chi_{c}^{2}$. This is achieved by extrapolating $\epsilon^{4} \alpha^{\prime}$ vs $\left\langle\Delta(-2 \ln L)_{\text {Null }}\right\rangle$ at $\Delta \chi_{c}^{2}$ for the generated $\epsilon^{4} \alpha^{\prime}$ values. An example sensitivity result is shown in Fig. 6.7 as the vertical dotted blue line.

SensMethod2: This method tries to employ the $\mathrm{CL}_{s}$ style CL technique $[35,100,101]$ that produces uncertainty on the sensitivity, but using the framework already developed by MiniBooNE. Instead of plotting $\epsilon^{4} \alpha^{\prime}$ vs. $\left\langle\Delta(-2 \ln L)_{\text {Null }}\right\rangle$ as done in SensMethod1, a predicted $\epsilon^{4} \alpha^{\prime}$ vs. $\left\langle\Delta(-2 \ln L)_{\text {Null }}\right\rangle$ distribution is simulated by randomly choosing a $\Delta(-2 \ln L)_{\text {Null }}$ value for each $\epsilon^{4} \alpha^{\prime}$ value based off its PDF (see Fig. 6.8b). For the predicted distribution the corresponding $\left(\epsilon^{4} \alpha^{\prime}\right)_{\text {Sens }}$ is calculated. 5000 predicted $\left(\epsilon^{4} \alpha^{\prime}\right)_{\text {Sens }}$ values are calculated from 5000 predicted $\epsilon^{4} \alpha^{\prime}$ vs. $\left\langle\Delta(-2 \ln L)_{\text {Null }}\right\rangle$ distributions. Fig. 6.9 gives an example cumulative distribution function $(\mathrm{CDF})$ of $\left(\epsilon^{4} \alpha^{\prime}\right)_{\text {Sens }}$. The $\left(\epsilon^{4} \alpha^{\prime}\right)_{\text {Sens }}$ distribution can be fitted with a Gaussian, but the distribution, as shown in Fig. 6.8b may not be symmetric. $\left(\epsilon^{4} \alpha^{\prime}\right)_{\text {Sens }}$ for the combination of $m_{V}, m_{\chi}$ is calculated to be when the $\mathrm{CDF}=0.5$. The $\pm 1 \sigma$ are when $\mathrm{CDF}=0.5 \pm \approx 0.34$ and $\pm 2 \sigma$ are when $\mathrm{CDF}$ $=0.5 \pm \approx 0.475$. Calculating the mean and uncertainty this way will better model the potential asymmetric behavior of $\left(\epsilon^{4} \alpha^{\prime}\right)_{\text {Sens }}$

Fig. 6.10 shows the comparison between SensMethod1 and SensMethod2 for two slices of the $m_{V}, m_{\chi}, \epsilon, \alpha^{\prime}$ parameter space. SensMethod2 is stronger, a.k.a lower, than SensMethod1 but does contain SensMethod1 within $1 \sigma$. SensMethod2 can be "biased" to producing a stronger sensitivity than SensMethod1 because there is no $\epsilon^{4} \alpha^{\prime}$ that will produce all fake data sets with $\Delta(-2 \ln L)_{\text {Null }}<\Delta \chi_{c}^{2}$. Since SensMethod1 falls within $1 \sigma$ of SensMethod2, SensMethod2 is the method used to produced the sensitivity for this analysis because it provides more information. 


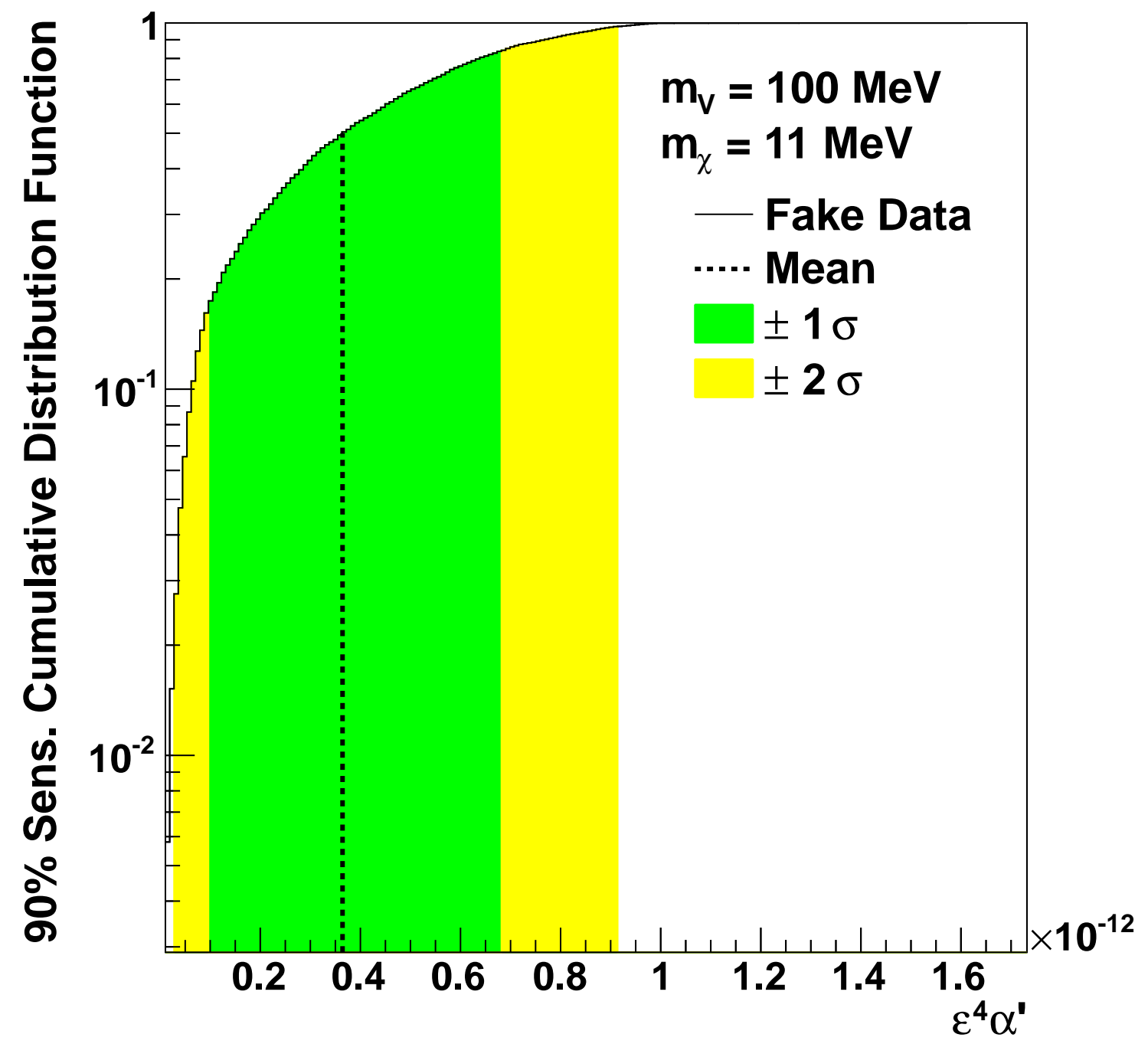

Figure 6.9: The corresponding CDF where the vertical black line is the calculated mean and the green (yellow) regions are the $\pm 1 \sigma( \pm 2 \sigma)$ calculated limits. 


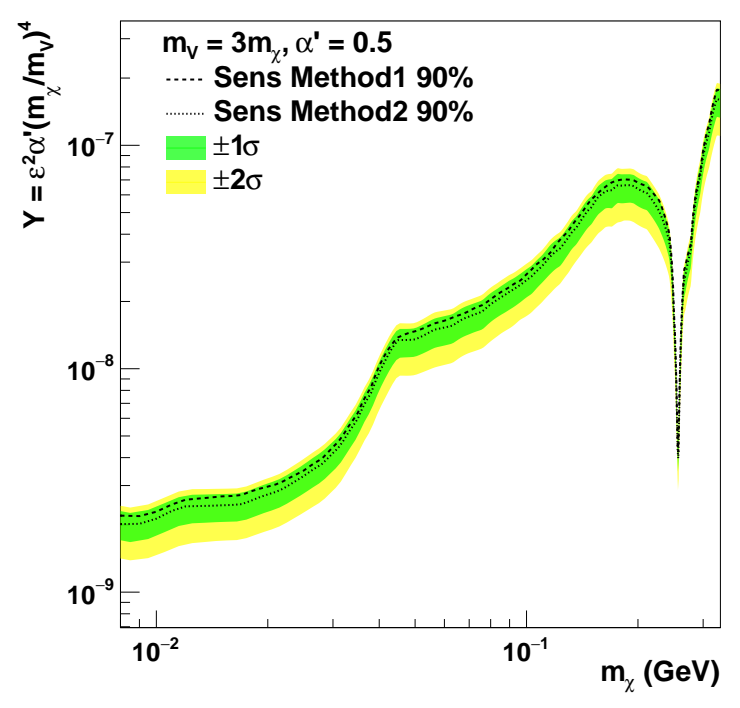

(a) $Y$ vs. $m_{\chi}$

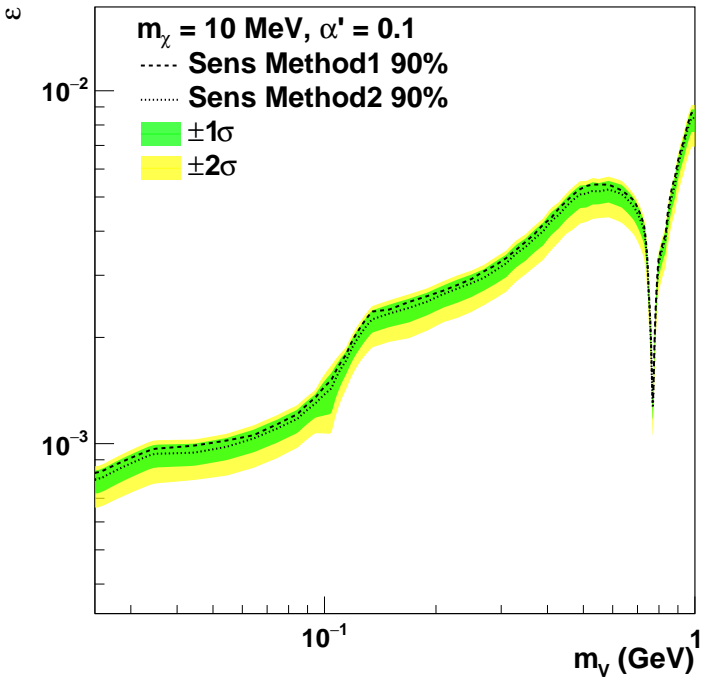

(b) $\epsilon$ vs. $m_{V}$

Figure 6.10: Comparing SensMethod1 and SensMethod2 for (a) $Y$ vs. $m_{\chi}$ with $m_{V}=3 m_{\chi}$ and $\alpha^{\prime}=0.5$, and (b) $\epsilon$ vs. $m_{V}$ with $m_{\chi}=10 \mathrm{MeV}$ and $\alpha^{\prime}=0.1$.

\section{GENERATING CONFIDENCE LIMITS}

All the fake data sets needed for generating the CL were already generated when calculating the sensitivity. For each fake data set $\Delta(-2 \ln L)_{\operatorname{Sig}}$ is calculated. For each $\epsilon^{4} \alpha^{\prime}$ $\Delta(-2 \ln L)_{\text {Sig }}$ distribution $\Delta \chi_{c}^{2}$ is calculated. An example of this is shown by black histogram in Fig. 6.7. The resulting $\Delta \chi_{c}^{2}$ distribution is fitted by a 0th-order polynomial since the distribution of $\Delta \chi_{c}^{2}$ for a given $m_{V}, m_{\chi}$ combination is nearly independent of $\epsilon^{4} \alpha^{\prime} . \Delta(-2 \ln L)_{\text {Sig }}$ is then calculated using data instead of fake data (the black points in Fig. 6.7), and the CL is where data's $\Delta(-2 \ln L)_{\mathrm{Sig}}$ intersects the 0th-order polynomial. The 90\% CL using the fake data sets are shown in Fig. 6.12b.

Fig. 6.11 compares the two CL methods and the two sensitivity methods for two slices in the LDM parameter space. The CL set by the traditional $\chi^{2}$ method is stronger than the frequentist method because the fake data shows the effective number of degrees of freedom is larger than one. The traditional $\chi^{2}$ method also differs from SensMethod2 by 


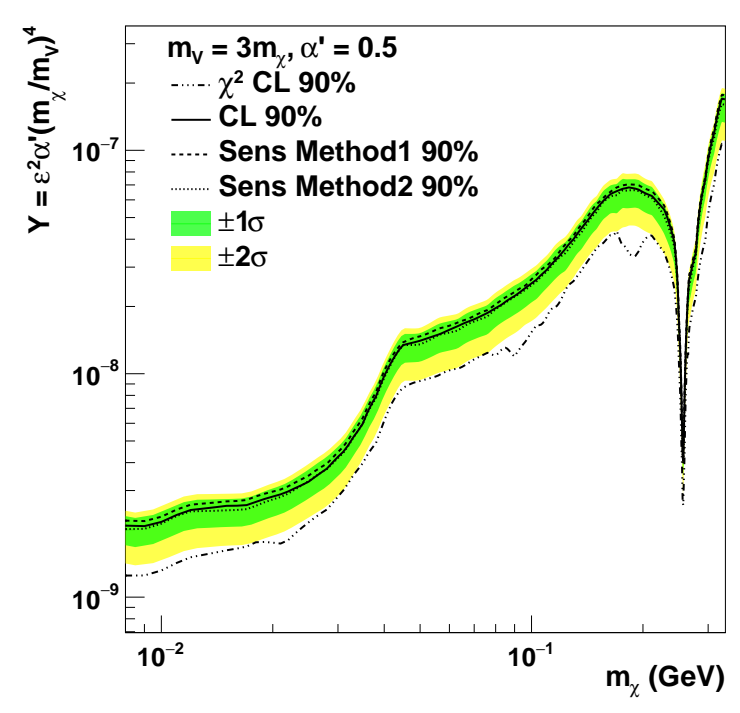

(a) $Y$ vs. $m_{\chi}$

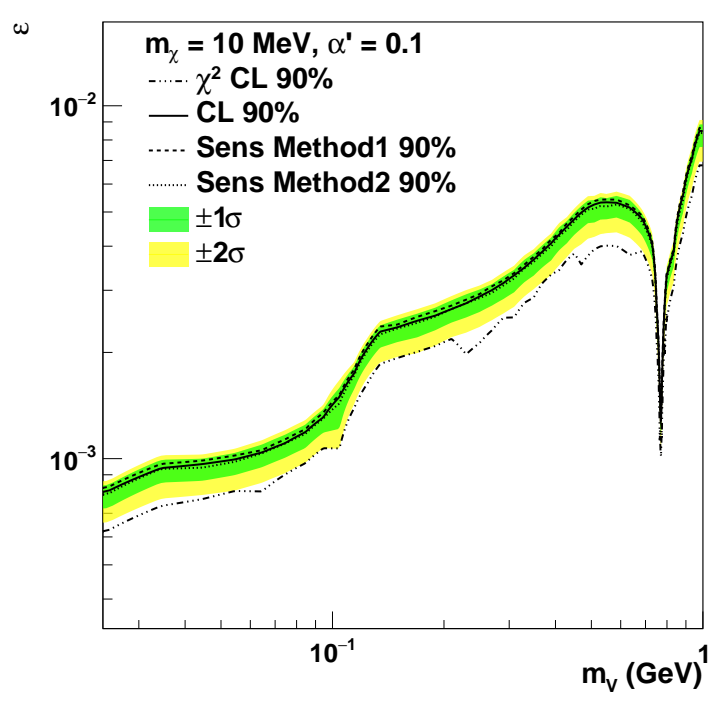

(b) $\epsilon$ vs. $m_{V}$

Figure 6.11: Comparing the two CL and sensitivity methods for (a) $Y$ vs. $m_{\chi}$ with $m_{V}=$ $3 m_{\chi}$ and $\alpha^{\prime}=0.5$, and (b) $\epsilon$ vs. $m_{V}$ with $m_{\chi}=10 \mathrm{MeV}$ and $\alpha^{\prime}=0.1$.

more than $2 \sigma$. Fig. 6.12 gives the CL and sensitivity results for both the frequentist and the traditional methods for the entire parameter space. The best limit is at $m_{V}=769 \mathrm{MeV}$ and $m_{\chi}=381 \mathrm{MeV}$ with a $90 \% \mathrm{CL}$ of $\epsilon^{4} \alpha^{\prime}=1.2 \times 10^{-14}$. Assuming $\alpha^{\prime}=0.1$ the $90 \% \mathrm{CL}$ on $\epsilon=6.0 \times 10^{-4}$ at the best limit. These values of $m_{V}, m_{\chi}$ produce a ratio of $m_{\chi}$ to $m_{V}$ equal to 0.495 .

Taking the best fit $m_{V}, m_{\chi}$ point, a constrained fit test was done to see how probable data matches prediction assuming signal. Fig. 6.13 gives the probability that a signal fit to data is consistent with background plus signal, using Sec. 5.2.1 as the test statistic, to be $19 \%$. 


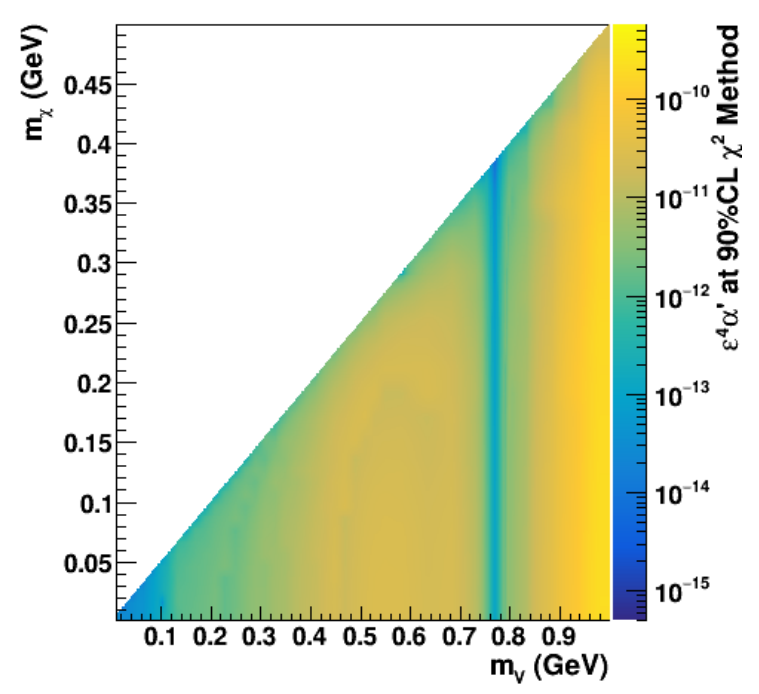

(a) Traditional

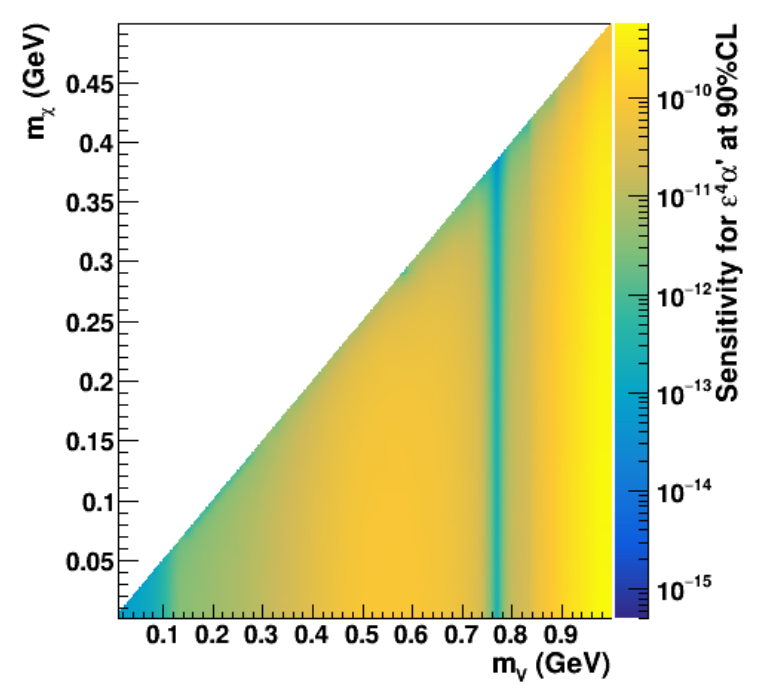

(c) Sensitivity SensMethod1

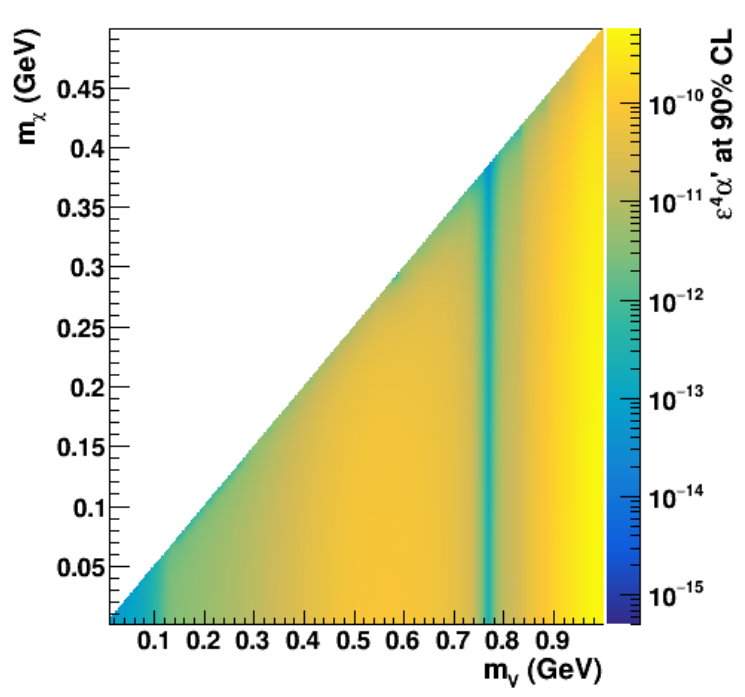

(b) Frequentist

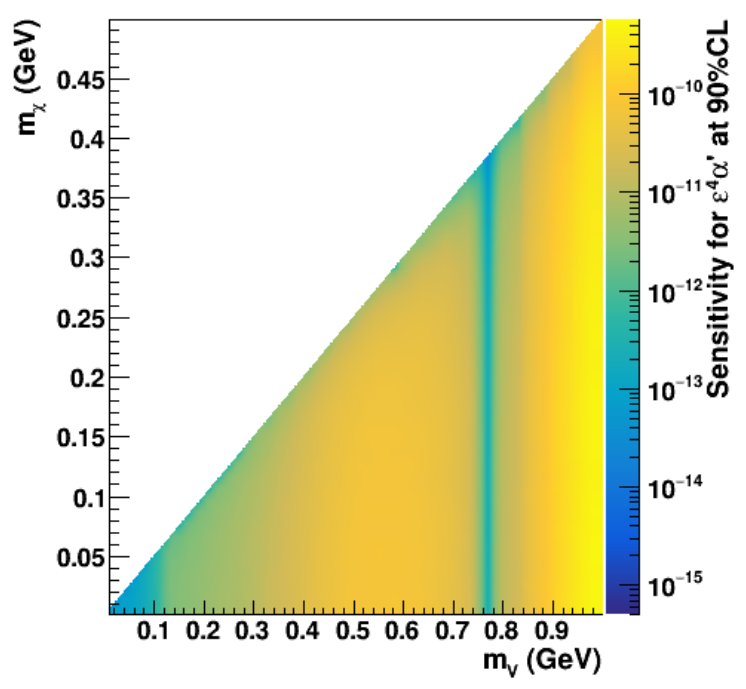

(d) Sensitivity SensMethod2

Figure 6.12: The $90 \% \mathrm{CL}$ on $\epsilon^{4} \alpha^{\prime}$ for each set of $m_{V}, m_{\chi}$ from using the (a) traditional and (b) frequentist approaches. The sensitivity is shown in (c) for SensMethod1 and (d) for SensMethod2. 


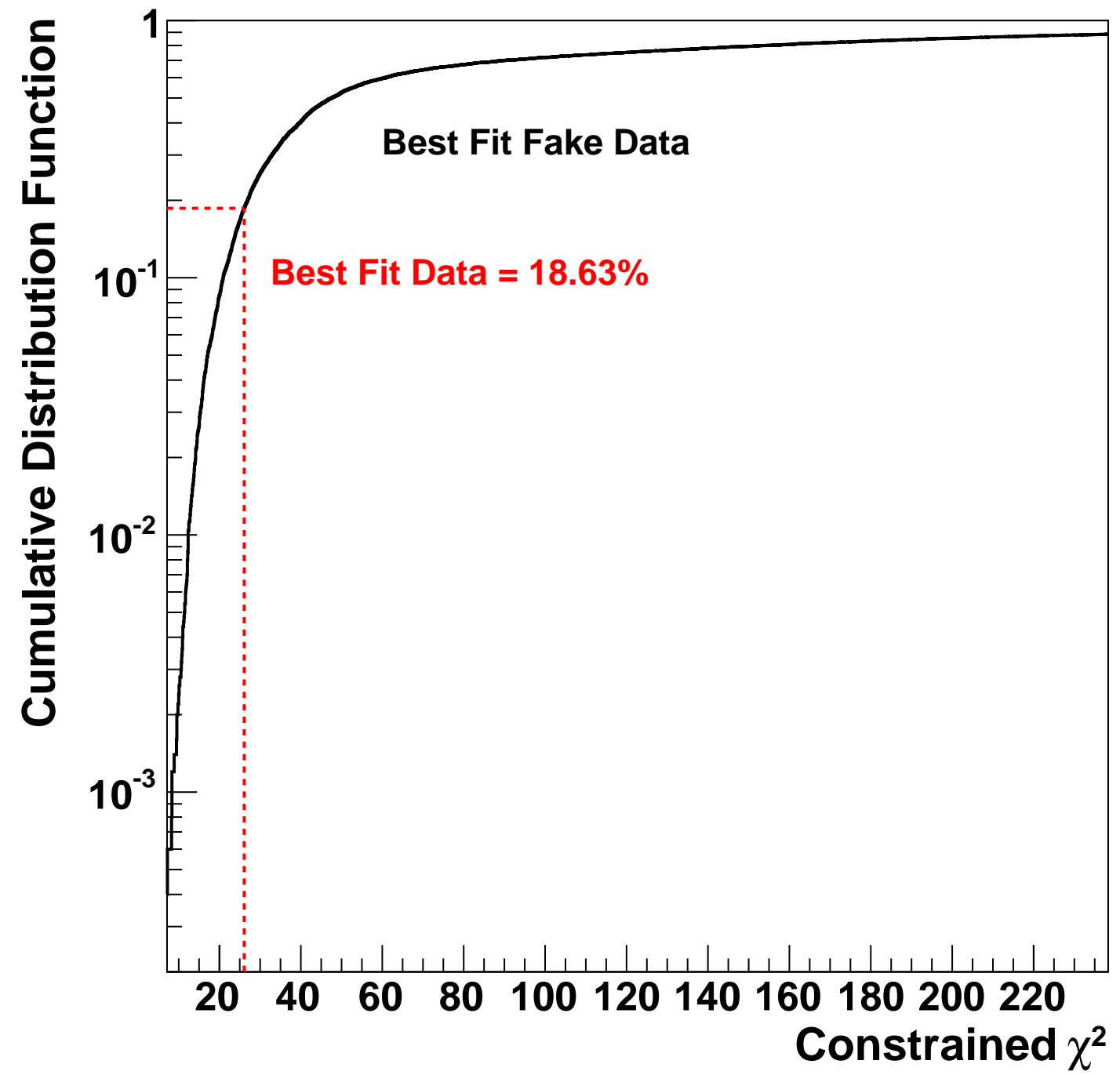

Figure 6.13: The CDF function for signal fake data where the signal parameters are set to the overall best fit results and the result from the signal best fit constrained fit. The probability that data is consistent with background predictions plus best fit dark matter using the constrained fit is $19 \%$. 


\section{CHAPTER 7}

\section{CONCLUSION}

MiniBooNE conducted a dedicated dark matter (DM) search with a special "off-target" run where the Booster Neutrino Beamline protons were steered off-target to reduce the neutrino flux by 26.2 , and made the neutrino spectrum softer by $176 \mathrm{MeV}$ compared to $\nu$-Mode running (see Sec. 4.2). No significant excess over background was observed and a 90\% confidence limit (CL) was placed on the vector portal light dark matter (LDM) model using a frequentist approach (see Sec. 6.2.2 and Fig. 7.1).

Since the LDM model consists of four free parameters (see Sec. 2.3), slices of the LDM parameter space are needed to compare to other experiments. Figs. 7.2 and 7.3 compares this analysis with previous results. Fig. 7.2 plots $Y$, the cross section scaling variable (see Sec. 2.3), verses $m_{\chi}$ where $m_{V}$ is set to three times $m_{\chi}$ and $\alpha^{\prime}=0.5$. Fig. 7.3 plots $\epsilon$ verses $m_{V}$ where $m_{\chi}$ is set to $10 \mathrm{MeV}$ and $\alpha^{\prime}=0.1$. These are standard slices to present in the LDM community.

In all parameter space tested, MiniBooNE excludes this model as a solution to the muon g-2 discrepancy (see Sec. 2.3.1). MiniBooNE excludes new parameter space primarily when $100<m_{\chi}<400 \mathrm{MeV}$, At low to medium $m_{\chi} / m_{V}$ ratio MiniBooNE excludes where this model solely satisfies the DM relic density. All previous curves, including the direct detection results, are reinterpretations of the published results.

Model independent fit (MIF) results have also been obtained by doing a general excess 


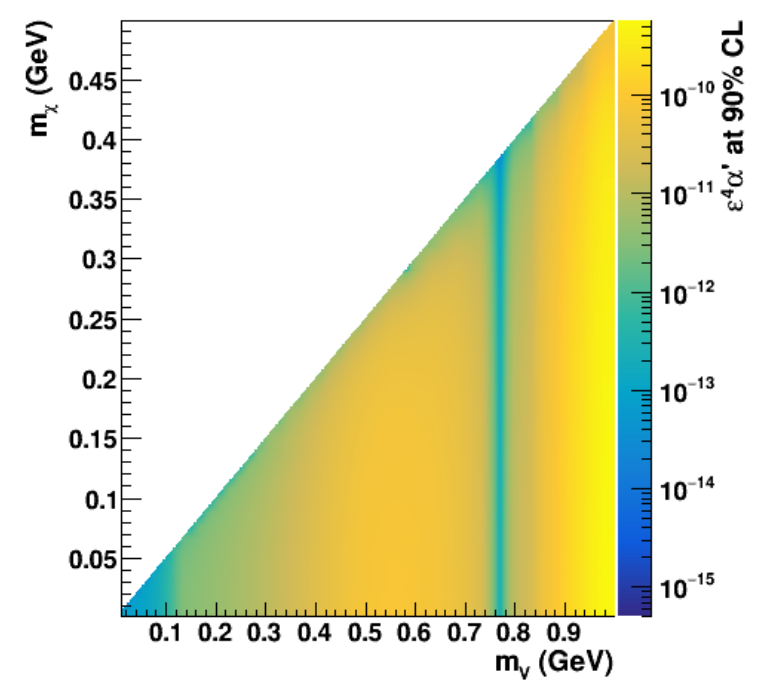

(a) Confidence limit (Frequentist)

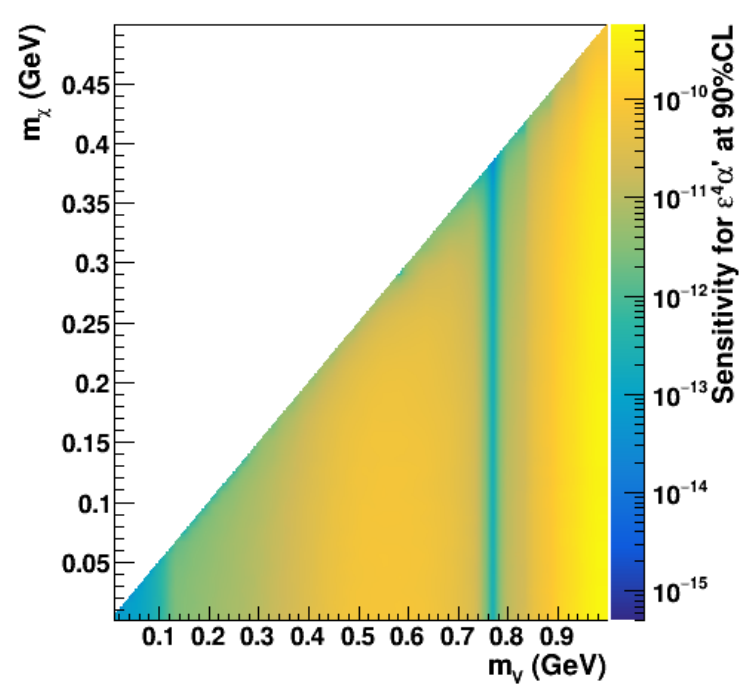

(b) Sensitivity (SensMethod2)

Figure 7.1: (a) The $90 \% \mathrm{CL}$ on $\epsilon^{4} \alpha^{\prime}$ for each set of $m_{V}, m_{\chi}$ from using the frequentist approach discussed in Sec. 6.2. (b) The $90 \%$ sensitivity on $\epsilon^{4} \alpha^{\prime}$ for the same combinations of $m_{V}, m_{\chi}$ as in (a).

search (see Sec. 5.3). The MIF found (130 \pm 270$)$ excess events/(1e20 protons on target) when $0.1 \leq T_{N}^{\text {true }}<2 \mathrm{GeV}$. The data and covariance matrix from the MIF have been used to set limits on a variation of the vector portal LDM model, called leptophobic LDM. In this model, interactions with leptons is highly suppressed by adding a baryonic current $\left(q_{B} V_{\mu} J_{B}^{\mu}\right)$ to Eq. (2.4) and setting $\epsilon=0$. Fig. 7.4 shows MiniBooNE's $90 \%$ CL in the leptophobic LDM model using the MIF results. In the leptophobic LDM model MiniBooNE excludes a large range of new parameter space. Experiments on proton fixed-target beamlines have high sensitivity to the leptophobic LDM by searching for neutral-current elastic (NCE) and inelastic $\Delta$ signatures in their detector. MiniBooNE has produced neutralcurrent inelastic $\pi^{0}$ cross sections, primarily from $\Delta$ decay, from neutrino and anti-neutrino scattering [111], and plans on producing a LDM search results in this channel. The neutralcurrent inelastic $\pi^{0}$ interaction composes only $8 \%$ of the total neutrino production in MiniBooNE, compared to $16 \%$ from the NCE channel [69]. This analysis will not benefit from 


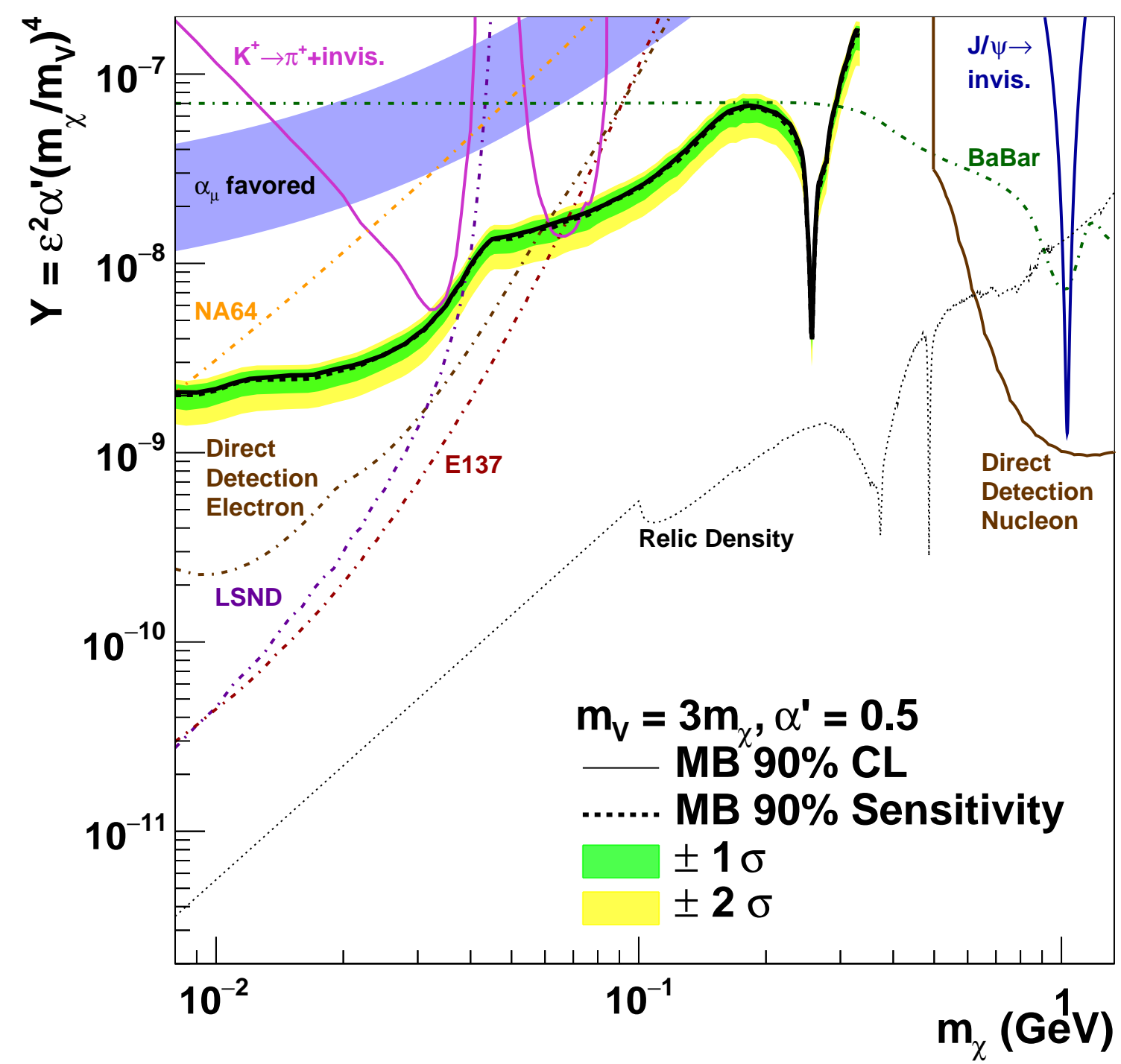

Figure 7.2: Slices of Fig. 6.12 in $Y$ vs. $m_{\chi}$ with a fixed ratio of $m_{\chi} / m_{V}$ and fixed $\alpha^{\prime}$. The blue shaded region is where this model reduces the muon g-2 discrepancy between theory and experiment to $\pm 3 \sigma[29-31]$. E137 was an electron beam dump at SLAC originally searching for axions $[53,55]$. LSND was a stop pion neutrino detector at LANCE $[25,46$, 102]. Direct detection includes limits from the most sensitive low mass results, currently from CDMSlight [103] and CRESST-II [34] for nucleon recoils and Xenon10 [104] for electron recoil. $K^{+} \rightarrow \pi^{+}+$invisible was a rare kion decay search done at BNL (E949) [32, $53,105] . J / \Psi \rightarrow$ invisible was done by BES searching for missing energy in the $J / \Psi$ decay [106]. BaBar was a reinterpretation of $\Upsilon(3 S) \rightarrow \gamma+$ invisible [107, 108]. NA64 searched for missing energy [109]. Dot-dashed lines rely on DM coupling to leptons. 


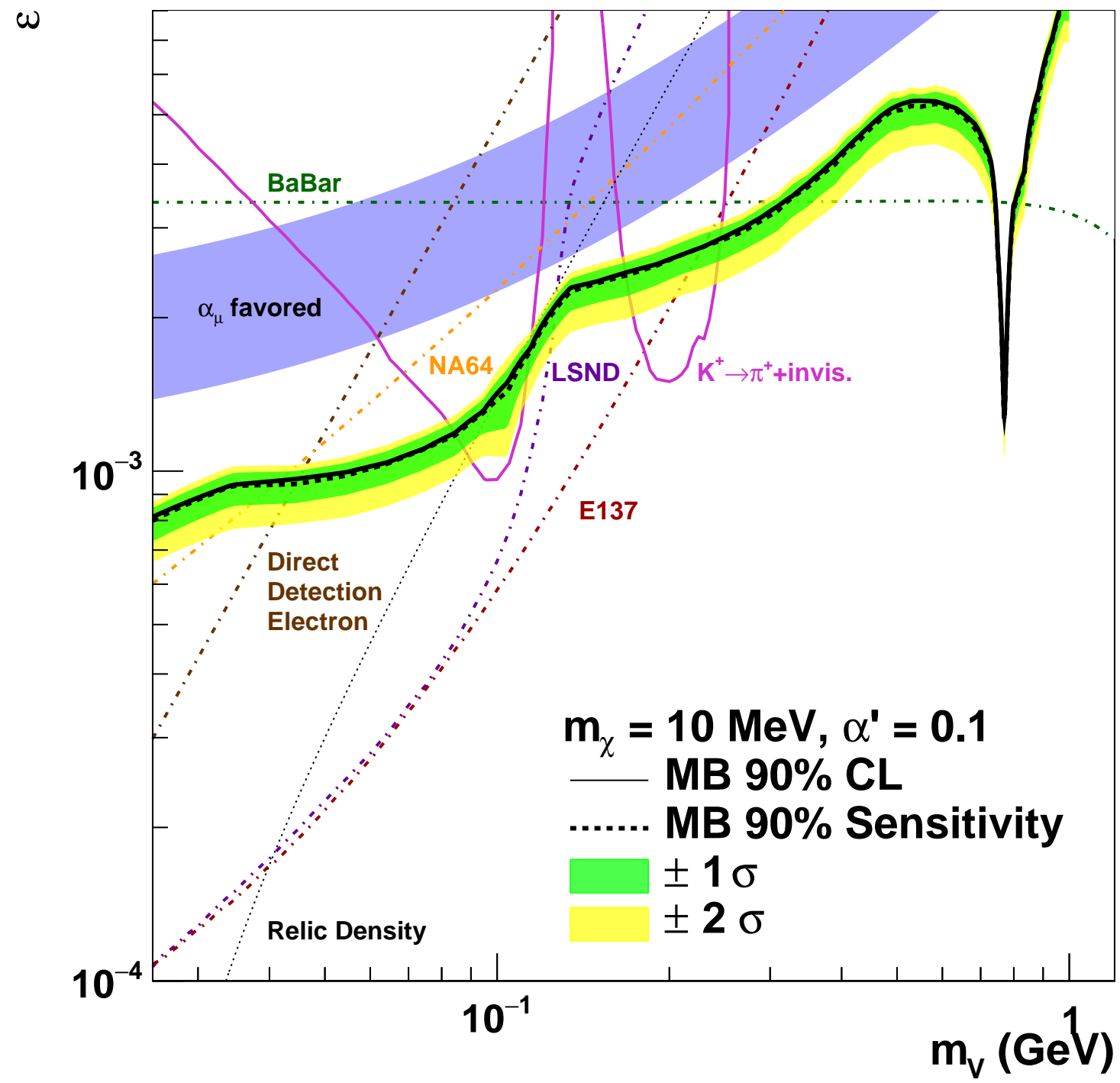

Figure 7.3: Slices of Fig. 6.12 in $\epsilon$ vs. $m_{V}$ with a fixed $m_{\chi}$ and $\alpha^{\prime}$. The blue shaded region is where this model reduces the muon g-2 discrepancy between theory and experiment to $\pm 3 \sigma[29-31]$. E137 was an electron beam dump at SLAC originally searching for axions [53, 55]. LSND was a stop pion neutrino detector at $\operatorname{LANCE}[25,46,102] . K^{+} \rightarrow \pi^{+}+$invisible was a rare kion decay search done at BNL (E949) [32, 53, 105]. BaBar was a reinterpretation of $\Upsilon(3 S) \rightarrow \gamma+$ invisible [107, 108]. NA64 searched for missing energy [109]. Direct detection electron is from Xenon10 [104]. Dot-dashed lines rely on DM coupling to leptons. 


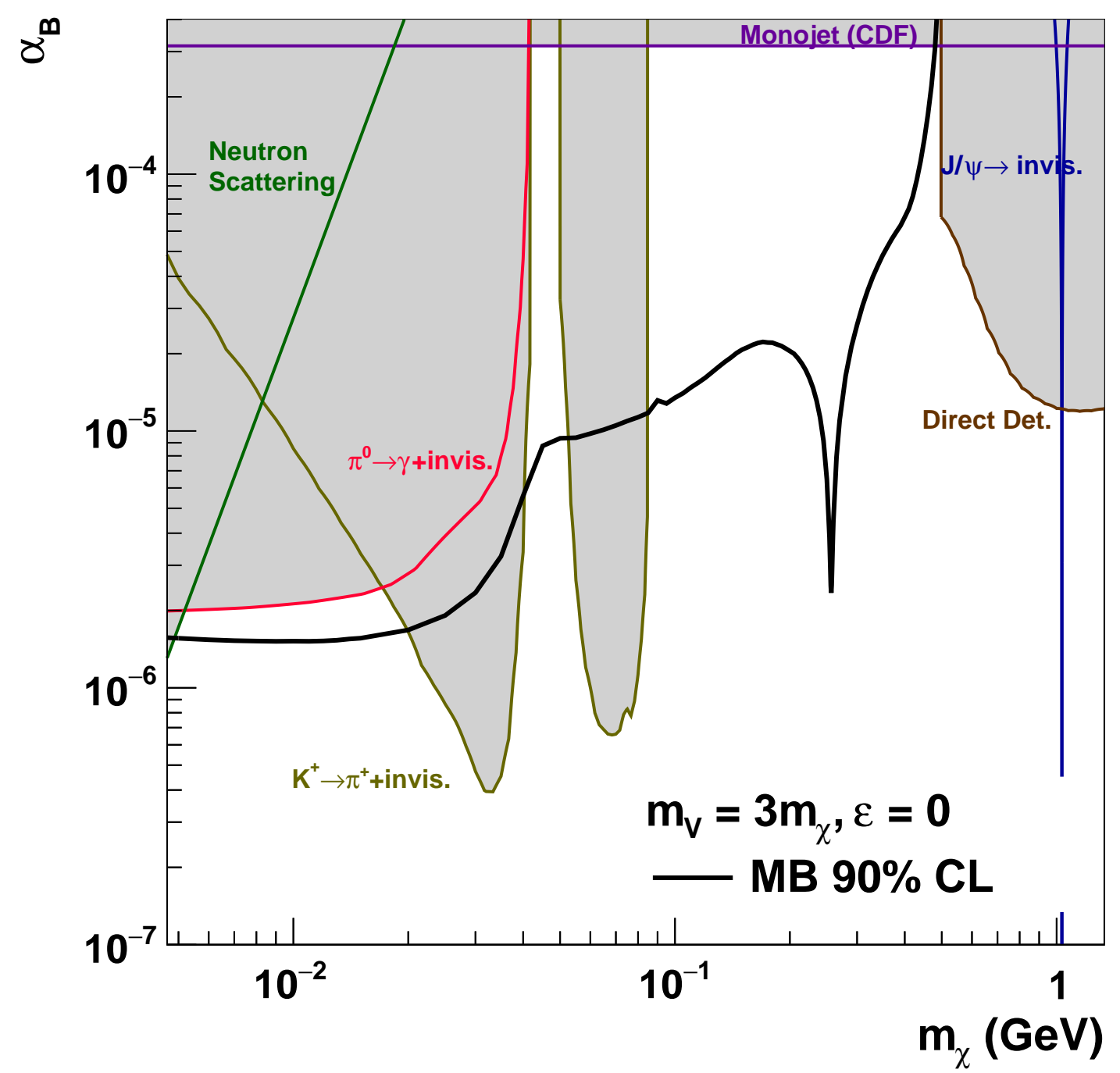

Figure 7.4: Leptophobic 90\% CL using the MIF results. Explanation of the previous limits are given in Ref. [110]. 
more statistics because of it is systematic-limited (see Table 4.8), but more running would help statistical limit analyses such as searching for DM in the $\pi^{0}$ or electron channels.

The biggest improvement that can be made to this analysis is to replace the beryllium target/aluminum horn with a thick steel target. Simulations suggests this will reduce the charged-current quasielastic events in the MiniBooNE detector by about a thousand, and, therefore, reduce all neutrino channels by about the same amount. The implications for a NCE DM search would be to now have a region $T_{N}^{\text {reco }} \leq 300 \mathrm{MeV}$ where the only contributing background would be beam-unrelated background. This would greatly increase MiniBooNE's sensitivity to DM because beam-unrelated background is well measured.

MiniBooNE has conducted the first dedicated proton beam dump DM search, setting a path for continuing DM searches, on the Booster Neutrino Beamline and elsewhere. 


\section{BIBLIOGRAPHY}

[1] F. Zwicky, "Die Rotverchiebung von extraglaktischen", Helv. Phys. Acta 6, 110 (1933).

[2] S. Smith, "The Mass of the Virgo Cluster", Astrophysical Journal 83, 23 (1936).

[3] T. S. van Albada, J. N. Bahcall, K. Begeman, and R. Sancisi, "Distribution of dark matter in the spiral galaxy NGC 3198", Astrophysical Journal 295, 305 (1985).

[4] G. Bertone, D. Hooper, and J. Silk, "Particle dark matter: Evidence, candidates and constraints", Phys.Rept. 405, 279 (2005), arXiv:hep-ph/0404175 [hep-ph] .

[5] R. Adam et al., "Planck 2015 results. I. Overview of products and scientific results", Astron. Astrophys. 594, A1 (2016), arXiv:1502.01582 [astro-ph.CO].

[6] S. Profumo, "Astrophysical Probes of Dark Matter", in Proceedings, Theoretical Advanced Study Institute in Elementary Particle Physics: Searching for New Physics at Small and Large Scales (TASI 2012): Boulder, Colorado, June 4-29, 2012 (2013), pp. 143-189, arXiv:1301.0952 [hep-ph].

[7] P. A. R. Ade et al., "Planck 2015 results. XIII. Cosmological parameters", Astron. Astrophys. 594, A13 (2016), arXiv:1502.01589 [astro-ph.C0].

[8] E. Hupp, D. Brown, S. Roy, and M. Watzke, NASA Finds Direct Proof of Dark Matter, NASA RELEASE 06-297, (Aug. 2006) http://chandra.harvard.edu/press/06_releases/press_082106.html.

[9] T. Falk, K. A. Olive, and M. Srednicki, "Heavy sneutrinos as dark matter", Phys. Lett. B339, 248 (1994), arXiv:hep-ph/9409270 [hep-ph].

[10] T. Moroi, H. Murayama, and M. Yamaguchi, "Cosmological constraints on the light stable gravitino", Phys. Lett. B303, 289 (1993). 
[11] W. Buchmuller, K. Hamaguchi, and M. Ratz, "Gauge couplings at high temperature and the relic gravitino abundance", Phys. Lett. B574, 156 (2003), arXiv:hep-ph/0307181 [hep-ph] .

[12] K. Choi, K. Hwang, H. B. Kim, and T. Lee, "Cosmological gravitino production in gauge mediated supersymmetry breaking models", Phys. Lett. B467, 211 (1999), arXiv:hep-ph/9902291 [hep-ph].

[13] T. Gherghetta, G. F. Giudice, and A. Riotto, "Nucleosynthesis bounds in gauge mediated supersymmetry breaking theories", Phys. Lett. B446, 28 (1999), arXiv:hep-ph/9808401 [hep-ph].

[14] S. A. Bonometto, F. Gabbiani, and A. Masiero, "Mixed dark matter from axino distribution", Phys. Rev. D49, 3918 (1994), arXiv:hep-ph/9305237 [hep-ph].

[15] T. Goto and M. Yamaguchi, "Is axino dark matter possible in supergravity?", Phys. Lett. B276, 103 (1992).

[16] C. Weinheimer, "The Neutrino mass direct measurements", in Neutrino telescopes. Proceedings, 10th International Workshop, Venice, Italy, March 11-14, 2003. Vol. 1+2 (2003), pp. 335-344, arXiv:hep-ex/0306057 [hep-ex] .

[17] S. Dodelson and L. M. Widrow, "Sterile-neutrinos as dark matter", Phys. Rev. Lett. 72, 17 (1994), arXiv:hep-ph/9303287 [hep-ph].

[18] X.-D. Shi and G. M. Fuller, "A New dark matter candidate: Nonthermal sterile neutrinos", Phys. Rev. Lett. 82, 2832 (1999), arXiv:astro-ph/9810076 [astro-ph].

[19] C. Boehm and P. Fayet, "Scalar dark matter candidates", Nucl.Phys. B683, 219 (2004), arXiv:hep-ph/0305261 [hep-ph].

[20] C. Boehm et al., "MeV dark matter: Has it been detected?", Phys.Rev.Lett. 92, 101301 (2004), arXiv:astro-ph/0309686 [astro-ph]. 
[21] P. Jean et al., "Early SPI/INTEGRAL measurements of $511 \mathrm{keV}$ line emission from the 4th quadrant of the Galaxy", Astron. Astrophys. 407, L55 (2003), eprint: astro-ph/0309484.

[22] J. Knodlseder et al., "Early SPI / INTEGRAL contraints on the morphology of the $511 \mathrm{keV}$ line emission in the 4th galactic quadrant", Astron. Astrophys. 411, L457 (2003), arXiv:astro-ph/0309442 [astro-ph].

[23] M. Pospelov, A. Ritz, and M. B. Voloshin, "Secluded WIMP Dark Matter", Phys.Lett. B662, 53 (2008), arXiv:0711.4866 [hep-ph].

[24] B. Batell, M. Pospelov, and A. Ritz, "Exploring Portals to a Hidden Sector Through Fixed Targets", Phys.Rev. D80, 095024 (2009), arXiv:0906.5614 [hep-ph].

[25] P. deNiverville, M. Pospelov, and A. Ritz, "Observing a light dark matter beam with neutrino experiments", Phys.Rev. D84, 075020 (2011), arXiv:1107.4580 $[$ hep-ph]

[26] P. deNiverville, D. McKeen, and A. Ritz, "Signatures of sub-GeV dark matter beams at neutrino experiments", Phys.Rev. D86, 035022 (2012), arXiv:1205.3499 $[$ hep-ph] .

[27] B. W. Lee and S. Weinberg, "Cosmological Lower Bound on Heavy Neutrino Masses", Phys.Rev.Lett. 39, 165 (1977).

[28] P. deNiverville, C.-Y. Chen, M. Pospelov, and A. Ritz, "Light dark matter in neutrino beams: production modelling and scattering signatures at MiniBooNE, T2K and SHiP", (2016), arXiv:1609.01770 [hep-ph].

[29] R. Bouchendira et al., "New Determination of the Fine Structure Constant and Test of the Quantum Electrodynamics", Physical Review Letters 106, 080801, 080801 (2011), arXiv:1012.3627 [physics.atom-ph]. 
[30] T. Aoyama, M. Hayakawa, T. Kinoshita, and M. Nio, "Tenth-Order QED Contribution to the Electron g-2 and an Improved Value of the Fine Structure Constant", Phys. Rev. Lett. 109, 111807 (2012), arXiv:1205.5368 [hep-ph].

[31] D. Hanneke, S. F. Hoogerheide, and G. Gabrielse, "Cavity Control of a Single-Electron Quantum Cyclotron: Measuring the Electron Magnetic Moment", Phys. Rev. A83, 052122 (2011), arXiv:1009.4831 [physics.atom-ph].

[32] M. Pospelov, "Secluded U(1) below the weak scale", Phys.Rev. D80, 095002 (2009), arXiv:0811.1030 [hep-ph].

[33] P. Fayet, "U-boson production in e+ e- annihilations, psi and Upsilon decays, and Light Dark Matter", Phys. Rev. D75, 115017 (2007), arXiv:hep-ph/0702176 $[\mathrm{HEP}-\mathrm{PH}]$.

[34] G. Angloher et al., "Results on light dark matter particles with a low-threshold CRESST-II detector", Eur. Phys. J. C76, 25 (2016), arXiv:1509.01515 [astro-ph.co].

[35] D. S. Akerib et al., "Results from a search for dark matter in LUX with 332 live days of exposure", (2016), arXiv:1608.07648 [astro-ph.C0].

[36] J. P. Lees et al., "Search for a Dark Photon in $e^{+} e^{-}$Collisions at BaBar", Phys. Rev. Lett. 113, 201801 (2014), arXiv:1406.2980 [hep-ex].

[37] A. Adare et al., "Search for dark photons from neutral meson decays in $p+p$ and $d$ + Au collisions at $\sqrt{s_{N N}}=200 \mathrm{GeV}$ ", Phys. Rev. C91, 031901 (2015), arXiv:1409.0851 [nucl-ex].

[38] S. Gardner, R. J. Holt, and A. S. Tadepalli, "New Prospects in Fixed Target Searches for Dark Forces with the SeaQuest Experiment at Fermilab", Phys. Rev. D93, 115015 (2016), arXiv:1509.00050 [hep-ph]. 
[39] S. Gninenko, "Constraints on sub-GeV hidden sector gauge bosons from a search for heavy neutrino decays", Phys.Lett. B713, 244 (2012), arXiv:1204. 3583 [hep-ph].

[40] G. Bernardi et al., "Search for Neutrino Decay", Phys.Lett. B166, 479 (1986).

[41] S. Gninenko, "Stringent limits on the $\pi^{0} \rightarrow \gamma \mathrm{X}, \mathrm{X} \rightarrow$ e+e- decay from neutrino experiments and constraints on new light gauge bosons", Phys.Rev. D85, 055027 (2012), arXiv:1112.5438 [hep-ph].

[42] C. Brown et al., "A New Limit on Axion Production in 800-GeV Hadronic Showers", Phys.Rev.Lett. 57, 2101 (1986).

[43] J. Blümlein and J. Brunner, "New Exclusion Limits on Dark Gauge Forces from Proton Bremsstrahlung in Beam-Dump Data", Phys.Lett. B731, 320 (2014), $\operatorname{arXiv:1311.3870~[hep-ph].~}$

[44] J. Blümlein and J. Brunner, "New Exclusion Limits for Dark Gauge Forces from Beam-Dump Data", Phys.Lett. B701, 155 (2011), arXiv:1104.2747 [hep-ex].

[45] J. Blümlein et al., "Limits on neutral light scalar and pseudoscalar particles in a proton beam dump experiment", Z.Phys. C51, 341 (1991).

[46] L. Auerbach et al., "Measurement of electron - neutrino - electron elastic scattering", Phys.Rev. D63, 112001 (2001), arXiv:hep-ex/0101039 [hep-ex].

[47] P. Astier et al., "Search for heavy neutrinos mixing with tau neutrinos", Phys.Lett. B506, 27 (2001), arXiv:hep-ex/0101041 [hep-ex].

[48] P. Adlarson et al., "Search for a dark photon in the $\pi^{0} \rightarrow e^{+} e^{-} \gamma$ decay", Phys.Lett. B726, 187 (2013), arXiv:1304.0671 [hep-ex].

[49] G. Agakishiev et al., "Searching a Dark Photon with HADES", Phys.Lett. B731, 265 (2014), arXiv:1311.0216 [hep-ex]. 
[50] A. A. Aguilar-Arevalo et al., "Measurement of the Neutrino Neutral-Current Elastic Differential Cross Section on Mineral Oil at $E_{\nu} \sim 1 \mathrm{GeV} "$, Phys. Rev. D82, 092005 (2010), arXiv:1007.4730 [hep-ex] .

[51] A. Aguilar-Arevalo et al., "Measurement of the Antineutrino Neutral-Current Elastic Differential Cross Section", Phys.Rev. D91, 012004 (2015), arXiv:1309.7257 [hep-ex].

[52] R. T. Thornton, "Accelerator-Produced Dark Matter Search using MiniBooNE", (2014), arXiv:1411.4311 [hep-ex].

[53] B. Batell, R. Essig, and Z. Surujon, "Strong constraints on Sub-GeV Dark Sectors from SLAC Beam Dump E137”, Phys.Rev.Lett. 113, 171802 (2014), arXiv:1406.2698 [hep-ph].

[54] J. D. Bjorken, R. Essig, P. Schuster, and N. Toro, "New Fixed-Target Experiments to Search for Dark Gauge Forces", Phys.Rev. D80, 075018 (2009), arXiv:0906.0580 [hep-ph].

[55] J. Bjorken et al., "Search for Neutral Metastable Penetrating Particles Produced in the SLAC Beam Dump", Phys.Rev. D38, 3375 (1988).

[56] E. Riordan et al., "A Search for Short Lived Axions in an Electron Beam Dump Experiment", Phys.Rev.Lett. 59, 755 (1987).

[57] A. Konaka et al., "Search for Neutral Particles in Electron Beam Dump Experiment", Phys.Rev.Lett. 57, 659 (1986).

[58] M. Davier, J. Jeanjean, and H. Nguyen Ngoc, "Search for Axions in Electron Bremsstrahlung", Phys.Lett. B180, 295 (1986).

[59] A. Bross et al., "A Search for Shortlived Particles Produced in an Electron Beam Dump", Phys.Rev.Lett. 67, 2942 (1991). 
[60] H. Merkel et al., "Search for Light Gauge Bosons of the Dark Sector at the Mainz Microtron", Phys.Rev.Lett. 106, 251802 (2011), arXiv:1101.4091 [nucl-ex].

[61] S. Abrahamyan et al., "Search for a New Gauge Boson in Electron-Nucleus Fixed-Target Scattering by the APEX Experiment", Phys.Rev.Lett. 107, 191804 (2011), arXiv:1108.2750 [hep-ex].

[62] A. Aguilar-Arevalo et al., "The Neutrino Flux prediction at MiniBooNE", Phys.Rev. D79, 072002 (2009), arXiv:0806.1449 [hep-ex].

[63] S. Agostinelli et al., "GEANT4: A Simulation toolkit", Nucl. Instrum. Meth. A506, 250 (2003).

[64] M. G. Catanesi et al., "Measurement of the production cross-section of positive pions in the collision of 8.9-GeV/c protons on beryllium", Eur. Phys. J. C52, 29 (2007), arXiv:hep-ex/0702024 [hep-ex].

[65] I. Chemakin et al., "Pion production by protons on a thin beryllium target at 6.4-Ge, 12.3-GeV/c, and 17.5-GeV/c incident proton momenta", Phys. Rev. C77, [Erratum: Phys. Rev.C77,049903(2008)], 015209 (2008), arXiv:0707.2375 [nucl-ex].

[66] J. R. Sanford and C. L. Wang, BNL Note 11299, 1967.

[67] C. Patrignani et al., "Review of Particle Physics", Chin. Phys. C40, 100001 (2016).

[68] A. A. Aguilar-Arevalo et al., "The MiniBooNE Detector", Nucl. Instrum. Meth. A599, 28 (2009), arXiv:0806.4201 [hep-ex].

[69] A. A. Aguilar-Arevalo et al., "First Measurement of the Muon Neutrino Charged Current Quasielastic Double Differential Cross Section", Phys. Rev. D81, 092005 (2010), arXiv:1002.2680 [hep-ex]. 
[70] A. Aguilar-Arevalo et al., "First measurement of the muon antineutrino double-differential charged-current quasielastic cross section", Phys.Rev. D88, 032001 (2013), arXiv:1301.7067 [hep-ex] .

[71] D. C. Cox, "A Measurement of the Neutral Current Neutrino-Nucleon Elastic Cross Section at MiniBooNE", PhD thesis (Indiana University, 2008).

[72] D. Casper, "The nuance neutrino physics simulation, and the future", Nuclear Physics B - Proceedings Supplements 112, 161 (2002).

[73] R. A. Smith and E. J. Moniz, "NEUTRINO REACTIONS ON NUCLEAR TARGETS", Nucl. Phys. B43, [Erratum: Nucl. Phys.B101,547(1975)], 605 (1972).

[74] C. H. Llewellyn Smith, "Neutrino Reactions at Accelerator Energies", Phys. Rept. 3, 261 (1972).

[75] K. Abe et al., "Measurement of the neutrino-oxygen neutral-current interaction cross section by observing nuclear deexcitation $\gamma$ rays", Phys. Rev. D90, 072012 (2014), arXiv:1403.3140 [hep-ex].

[76] R. Dharmapalan, "Antineutrino Neutral Current Interactions in MiniBooNE", PhD thesis (The University of Alabama, 2012).

[77] R. Brun et al., "GEANT3”, (1987).

[78] J. B. Birks, "Scintillations from Organic Crystals: Specific Fluorescence and Relative Response to Different Radiations", Proceedings of the Physical Society. Section A 64, 874 (1951).

[79] R. B. Patterson et al., "The Extended-track reconstruction for MiniBooNE", Nucl. Instrum. Meth. A608, 206 (2009), arXiv:0902.2222 [hep-ex].

[80] A. Bazarko et al., Studies of Scattering in Marcol 7 Mineral Oil, tech. rep. 144 (MiniBooNE, 2004). 
[81] D. Toptygin, Time-Resolved Fluorescence of MiniBooNE Mineral Oil, tech. rep. 122 (MiniBooNE, 2004).

[82] D. Perevalov, "Neutrino-Nucleus Neutral Current Elastic Interactions Measurement in MiniBooNE", PhD thesis (The University of Alabama, 2009).

[83] T. Katori, "A Measurement of the Muon Neutrino Charge Current Quasielastic Interaction and a Test of Lorentz Violation with the MiniBooNE Experiment", PhD thesis (Indiana University, 2008).

[84] R. T. Thornton, Pre-Trigger Activity Study for Dark Matter NCel Cuts, tech. rep. (MiniBooNE, 2015).

[85] E. Amaldi et al., "INCLUSIVE ETA PRODUCTION IN P P COLLISION AT ISR ENERGIES", Nucl. Phys. B158, 1 (1979).

[86] K. Jaeger et al., "Inclusive Gamma, pi0, K0 and Lambda Production in 12.4-GeV/c p p Interactions", Phys. Rev. D11, 1756 (1975).

[87] R. Brun and F. Rademakers, " $\{$ root $\}$ — an object oriented data analysis framework", Nuclear Instruments and Methods in Physics Research Section A: Accelerators, Spectrometers, Detectors and Associated Equipment 389, New Computing Techniques in Physics Research V, 81 (1997).

[88] D. Schmitz, "A measurement of hadron production cross-sections for the simulation of accelerator neutrino beams and a search for $\nu_{\mu} \rightarrow \nu_{e}$ oscillations in the $1 \mathrm{~m}^{2} \mathrm{eV}$ region", PhD thesis (Columbia, 2008).

[89] A. Aguilar-Arevalo, "An Improved Neutrino Oscillations Analysis of the MiniBooNE Data", PhD thesis (Columbia University, 2008).

[90] R. Patterson, "A search for Muon Neutrino to Electron Neutrino Oscillations at $\Delta m^{2}>1 e V^{2} ", \mathrm{PhD}$ thesis (Princeton University, 2007). 
[91] "Note Sur Une Méthode de Résolution des équations Normales Provenant de L'Application de la MéThode des Moindres Carrés a un Système D'équations Linéaires en Nombre Inférieur a Celui des Inconnues. - Application de la Méthode a la Résolution D'un Système Defini D'éQuations LinéAires", Bulletin géodésique 2, 67 (1924).

[92] G. H. Golub and C. F. V. Loan, Matrix Computations, 3rd (Johns Hopkins University Press, 1996).

[93] Z. Pavlovic, Likelihood Fit Method, BooNE Technical Note 291 (June 2010).

[94] M. Shaevitz, Constraining the $\nu_{e}$ Background Uncertainties with the Observed $\nu_{\mu}$ Events, BooNE Technical Note 221 (2007).

[95] M. Shaevitz, Nue Events with Numu Constraints, BooNE Technical Note 225 (2008).

[96] Wikipedia, Confidence interval - wikipedia, the free encyclopedia, [Online; accessed 4-January-2017], 2017.

[97] G. J. Feldman and R. D. Cousins, "A Unified approach to the classical statistical analysis of small signals", Phys. Rev. D57, 3873 (1998), arXiv:physics/9711021 [physics.data-an].

[98] A. G. Frodensen, O. Skjeggestad, and H. Tofte, Probability and Statistics in Particle Physics (Columbia University Press, 1979).

[99] S. S. Wilks, "The Large-Sample Distribution of the Likelihood Ratio for Testing Composite Hypotheses", Ann. Math. Statist. 9, 60 (1938).

[100] A. L. Read, "Presentation of search results: The CL(s) technique", J. Phys. G28, [,11(2002)], $2693(2002)$ 
[101] G. Cowan, K. Cranmer, E. Gross, and O. Vitells, "Asymptotic formulae for likelihood-based tests of new physics", Eur. Phys. J. C71, [Erratum: Eur. Phys. J.C73,2501(2013)], 1554 (2011), arXiv:1007.1727 [physics.data-an] .

[102] B. A. Dobrescu and C. Frugiuele, "GeV-Scale Dark Matter: Production at the Main Injector", JHEP 02, 019 (2015), arXiv:1410.1566 [hep-ph].

[103] R. Agnese et al., "New Results from the Search for Low-Mass Weakly Interacting Massive Particles with the CDMS Low Ionization Threshold Experiment", Phys. Rev. Lett. 116, 071301 (2016), arXiv:1509.02448 [astro-ph.CO].

[104] R. Essig et al., "First Direct Detection Limits on sub-GeV Dark Matter from XENON10”, Phys. Rev. Lett. 109, 021301 (2012), arXiv:1206.2644 [astro-ph. CO].

[105] A. V. Artamonov et al., "Study of the decay $K^{+} \rightarrow \pi^{+} \nu \bar{\nu}$ in the momentum region $140<P_{\pi}<199 \mathrm{MeV} /$ c", Phys. Rev. D79, 092004 (2009), arXiv:0903.0030 $[$ hep-ex].

[106] M. Ablikim et al., "Search for the invisible decay of J / psi in psi(2S) - pi+ pi- J / psi”, Phys. Rev. Lett. 100, 192001 (2008), arXiv:0710.0039 [hep-ex].

[107] B. Aubert et al., "Search for Invisible Decays of a Light Scalar in Radiative Transitions $v_{3 S} \rightarrow \gamma \mathrm{A} 0$ ", in Proceedings, 34th International Conference on High Energy Physics (ICHEP 2008): Philadelphia, Pennsylvania, July 30-August 5, 2008 (2008), arXiv:0808.0017 [hep-ex].

[108] R. Essig et al., "Constraining Light Dark Matter with Low-Energy $e^{+} e^{-}$ Colliders", JHEP 1311, 167 (2013), arXiv:1309.5084 [hep-ph].

[109] D. Banerjee et al., "Search for invisible decays of sub-GeV dark photons in missing-energy events at the CERN SPS", Phys. Rev. Lett. 118, 011802 (2017), arXiv:1610.02988 [hep-ex]. 
[110] B. Batell et al., "Leptophobic Dark Matter at Neutrino Factories", Phys. Rev. D90, 115014 (2014), arXiv:1405.7049 [hep-ph] .

[111] A. A. Aguilar-Arevalo et al., "Measurement of nu(mu) and anti-nu(mu) induced neutral current single pi0 production cross sections on mineral oil at $\mathrm{E}(\mathrm{nu}) \mathrm{O}(1-$ GeV)", Phys. Rev. D81, 013005 (2010), arXiv:0911.2063 [hep-ex]. 


\section{APPENDIX A}

\section{ENERGY-DEPENDENT $L_{t}^{p / e}$ CUT STUDY}

A study was done to see if a distinction could be made between neutrino neutral-current elastic (NCE) events and neutrino NCE-like events. A NCE-like event is where non-NCE neutrino interactions in the detector resulted in producing one and only one nucleon leaving the nucleus; no other particles are allowed to be admitted. These events are primarily from inelastic neutral-current pion production, where the pion get absorbed in the nucleus. Fig. A.1a shows there is a slight difference in the $p / e$ time log-likelihood ratio with the cut that was defined for both the $\nu$-Mode and $\bar{\nu}$-Mode analyses [76, 82], and Fig. A.1b shows that a more optimized cut could be made as a function of reconstructed kinetic energy. The figure of merit was defined as

$$
\mathcal{F}=\frac{\text { signal }}{\sqrt{\text { background }}}=\frac{\text { NUANCE channel }=2}{\sqrt{\mathrm{BUB}^{2}+\text { Dirt }^{2}+(\text { NUANCE channel } \neq 2)^{2}}} .
$$

Although the energy-dependent cut decreases the percentage of neutrino related backgrounds in $\mathrm{NCE}_{\mathrm{Off}}$ the energy independent cut was still decided upon because the gain in $\mathcal{F}$ was not large enough to merit the added complexity from the simple cut already determined for $\nu, \bar{\nu}$ analyses. 


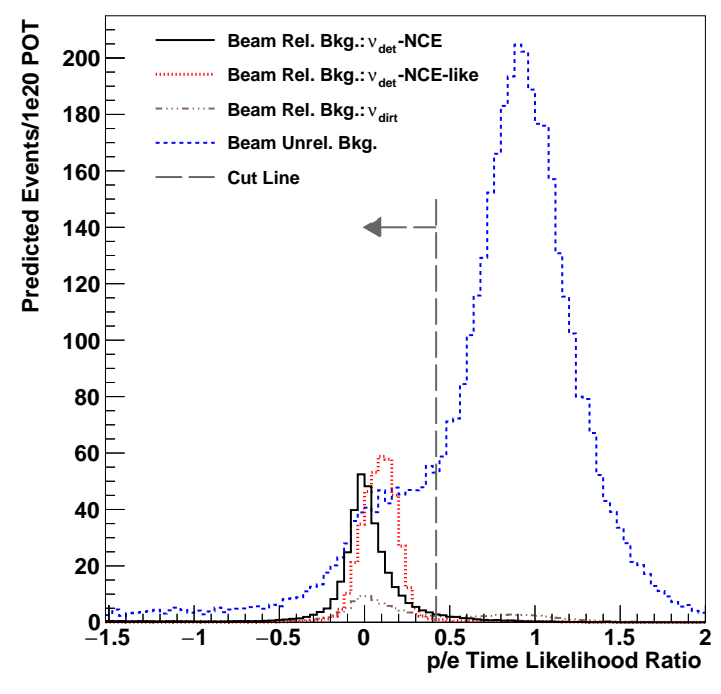

(a) $L_{t}^{p / e}$ Distribution

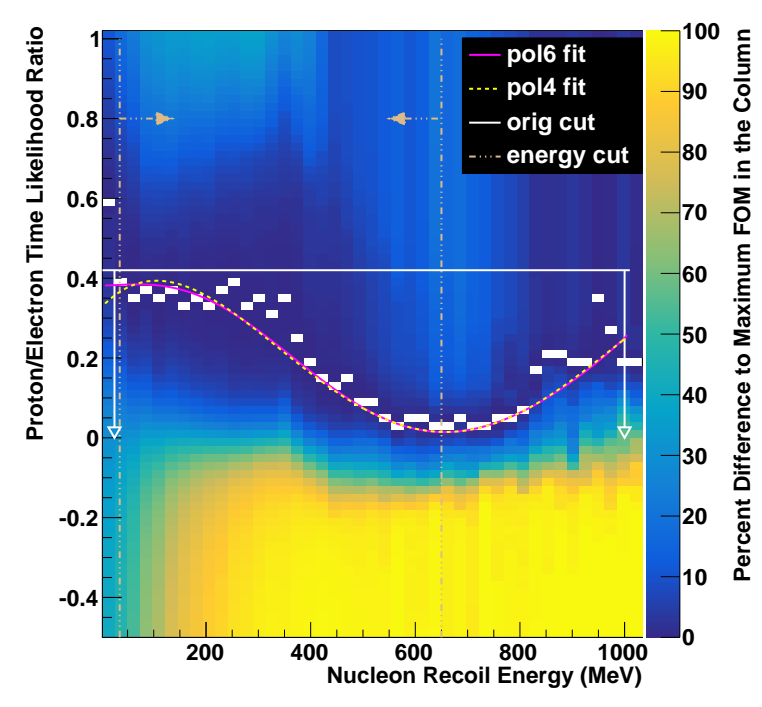

(b) Most Optimal Cut

Figure A.1: (a) The $L_{p / e}^{t}$ distribution broken down by channel; beam unrelated background, neutrino NCE interactions, neutrino NCE-like interactions, and neutrino interactions in the dirt. The vertical line and arrow shows where the cut is placed and which events are kept after the cut. All other cuts are applied. (b) $L_{t}^{p / e}$ vs $T_{N}^{r e c o}$ where the columns are differences to the minimum $\mathcal{F}$ in that column. The white line shows the original cut, the tan vertical lines show the $T_{N}^{r e c o}$ cut, the dashed yellow (magenta) line shows the results of fitting the minimum in each bin with a 4th- (6th-) order polynomial. 


\section{APPENDIX B}

\section{EFFECT OF CORRELATIONS ON FITS}

Consider the follow simple experiment. A measurement takes a measurement at $x_{1}$ and $x_{2}$ and records $y_{1}$ and $y_{2}$ respectively. The assumed underlying distribution is

$$
y(x)=\hat{y}
$$

The covariance matrix of $\hat{y}$ is assumed to be

$$
M=\left[\begin{array}{ll}
\alpha & \gamma \\
\gamma & \beta
\end{array}\right] .
$$

If the measurements are fitted with the fit function $f=\theta$, then the following questions are what we need to answer: (i) what is the best fit value $\theta_{\min }$ ?, and (ii) when is $\theta_{\min }$ $>\max \left(y_{1}, y_{2}\right)$ or $<\min \left(y_{1}, y_{2}\right) ?$

\section{B.1 FIT CALCULATIONS}

It can be shown that determinate of $\mathrm{M}|M|$ is always greater than 0 if the uncertainties and covariances are determined from an ensemble of experiments. We constrain $\alpha, \beta$ and $\gamma$ to make sure that is true causing the inverse of the matrix to exists. Using the $\chi^{2}$ method for produces

$$
\begin{aligned}
\chi^{2} & =\sum_{i=1}^{2} \sum_{j=1}^{2}\left(\theta-y_{i}\right) M_{i j}^{-1}\left(\theta-y_{j}\right) \\
& =\frac{1}{|M|}\left[\beta\left(\theta-y_{1}\right)^{2}-2 \gamma\left(\theta-y_{1}\right)\left(\theta-y_{2}\right)+\alpha\left(\theta-y_{2}\right)^{2}\right]
\end{aligned} .
$$


When fitting to the data the goal is to find the minimum $\chi^{2}$ value. Taking the first derivative of Eq. (B.1) with respect to $\theta$ and setting it equal to zero gives

$$
\theta_{\min }=\frac{\beta y_{1}+\alpha y_{2}-\gamma\left(y_{1}+y_{2}\right)}{\beta+\alpha-2 \gamma} .
$$

It can be shown that $\theta_{\min }$ is the minimum and not the maximum.

\section{B.2 CONDITIONS ON $\theta_{\min }$}

When $\operatorname{can} \theta_{\min }>y_{\max }$ and $\theta_{\min }<y_{\min }$ ? Using the fact that the denominator is positive, using the definition that $\alpha$ and $\beta$ are also positive, and subsituting Eq. (B.2) for $\theta_{\min }$ in the inequality gives

$$
\gamma>\frac{\beta\left(\frac{y_{1}}{y_{\max }}-1\right)+\alpha\left(\frac{y_{2}}{y_{\max }}-1\right)}{\frac{y_{1}}{y_{\max }}+\frac{y_{2}}{y_{\max }}-2} .
$$

We wrote the inequality in the form of Eq. (B.3) because it is easy to see that

$$
\gamma>\left\{\begin{array}{cc}
\alpha, & y_{\max }=y_{1} \\
\beta, & y_{\max }=y_{2}
\end{array} .\right.
$$

For physical data events where $\alpha$ and $\beta=\hat{y}$, this means the correlations must be bigger than the variance which is unphysical because of the following inequality $|\gamma|<\sqrt{\alpha \beta}$. Using this inequality and Eq. (B.4) we can say

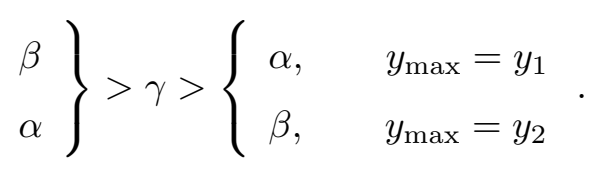

Similarly

$$
\left.\begin{array}{l}
\alpha \\
\beta
\end{array}\right\}>\gamma> \begin{cases}\beta, & y_{\min }=y_{1} \\
\alpha, & y_{\min }=y_{2}\end{cases}
$$

is the condition needed for the fit to be below both points.

Correlations have an effect on the fits. The best fit, independent on the strength of the corrections, occurs when the fit parameter falls the minimum number of standard deviations away in the uncorrelated basis. In the correlated basis in which the data was taken, this best fit may be above or below the data due to the relationship between the elements of the covariance matrix. 


\section{APPENDIX C}

\section{SINGULAR VALUE DECOMPOSITION}

A singular value decomposition of any matrix $A$ that is of size $(m \times n)$ with $m \geq n$ can be obtained by setting $A$ equal to

$$
A=U \Sigma V^{\top}
$$

where $U$ is a $(m \times m)$ matrix, $\Sigma$ is a diagonal $(m \times n)$ matrix and $V$ is a $(n \times n)$ matrix. To construct the $U, \Sigma$, and $V$ matrices one finds the eigenvalues $\lambda$ and eigenvectros $\boldsymbol{v}$ of $A^{\top} A$. Defing $\Sigma$ by

$$
\Sigma_{i i}=\sigma_{i}=\sqrt{\lambda_{i}} \mid \sigma_{1} \geq \sigma_{2} \geq \ldots \geq \sigma_{m}
$$

and $V$ by

$$
V=\left[\begin{array}{lll}
\boldsymbol{v}_{1} & \boldsymbol{v}_{2} \cdots \boldsymbol{v}_{m}
\end{array}\right]
$$

Using Eq. C.1 we can construct $\boldsymbol{u}_{i}=A \boldsymbol{v}_{i} / \sigma_{i}$ which makes

$$
U=\left[\begin{array}{lll}
\boldsymbol{u}_{1} & \boldsymbol{u}_{2} \cdots \boldsymbol{u}_{m}
\end{array}\right]
$$

the equation for $U$. By definition $U$ and $V$ are both column and row orthonormal which means that $U^{\top}=U^{-1}$ and $V^{\top}=V^{-1}$. The basis of $\Sigma$ is represented by $V$, so any $(n \times 1)$ vector $y$ that is in the same basis as $A$ can be rotated to the basis of $\Sigma$ by $V^{\top} y . \Sigma$ is an uncorrelated basis of $A$. 


\section{Curriculum Vitae}

\section{Remington Tyler Thornton}

\section{Research Interests}

Experimental neutrino physics: oscillations, cross sections, interactions

Experimental accelerator physics

Neutron induced fission: better understanding of daughter products and cross sections

\section{Education}

Indiana University

Ph.D., GPA - 3.861/4.0

Major: Nuclear Physics

Indiana University

Masters of Science, GPA - 3.767/4.0

Major: Physics

\section{Abilene Christian University}

Bachelors of Science, GPA - 3.48/4.0

Major: Computational Physics, Minor: Mathematics
Bloomington, IN

2013-2017

\section{Bloomington, IN}

2012-2013

Abilene, TX

2007-2011

\section{Ph.D. Thesis}

Title: Search for Light Dark Matter in a Proton Beam Dump

Supervisor: Professor Rex Tayloe

Description: A neutral current elastic nucleon signature in MiniBooNE from off-target running was used to search for accelerator-produced sub-GeV dark matter. A model inde- 
pendent result and a $90 \%$ confidence limit was put on the vector portal light dark matter model where new regions of phase space were excluded.

\section{Publications}

Collaborator: D. Akimov et al., "The COHERENT Experiment at the Spallation Neutron Source", (2015), arXiv:1509.08702 [physics.ins-det]

Author: R. T. Thornton, "Accelerator-Produced Dark Matter Search using MiniBooNE", (2014), arXiv:1411.4311 [hep-ex]

Collaborator: M. Heffner et al., "A Time Projection Chamber for High Accuracy and Precision Fission Cross Section Measurements", Nucl.Instrum.Meth. A759, 50 (2014),

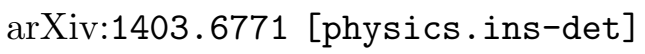

Collaborator: S. Brice et al., "A method for measuring coherent elastic neutrino-nucleus scattering at a far off-axis high-energy neutrino beam target", Phys.Rev. D89, 072004 (2014), arXiv:1311.5958 [physics.ins-det]

\section{Employment}

\section{Indiana University}

Graduate Research Assistant

Supervisor: Prof. Rex Tayloe

- The MiniBooNE experiment: Ph.D thesis

- SciBath: 3D tracking liquid scintillator neutral particle detector

\section{Indiana University}

Undergraduate Research Assistant

Supervisor: Prof. Rex Tayloe

○ SciBath
Bloomington, IN

Aug 2012 - Present

Bloomington, IN

Sept 2011 - Aug 2012

Abilene Christian University

Abilene, TX

Undergraduate Research Assistant

Supervisor: Prof. Rusty Towell
Jun 2008 - Dec 2011 
- Neutron Induced Fission Fragment Tracking Experiment (NIFFTE): Time Projection Chamber to measure back-to-back neutron induced fission

Idaho State University

Undergraduate Research Assistant

Supervisor: Dr. Eric Burgett

○ NIFFTE

\section{Lawrence Livermore National Laboratory}

Temporary Student Intern

Supevisor: Dr. Mike Heffner

○ NIFFTE

\section{Los Alamos National Laboratory}

Undergraduate Student ST 2

Supervisor: Dr. Fredrik Toversson

○ NIFFTE
Pocatello, ID

Jun 2011 - July 2011

Livermore, CA

Jun 2010 - Aug 2010

Los Alamos, NM

Jun 2009 - Aug 2009 


\section{Current Research Activities}

\section{MiniBooNE}

Fermilab, Batavia, IL

Feb 2014 - Present

MiniBooNE is a neutrino appearance oscillation search detector. A beam off-target run also took place to search for low-mass dark matter in 2013-2014. My job is to analyze the beam off-target data in search of a dark matter nucleon scattering signature. Technical Accomplishments include:

- Will produce the first thesis from the beam off-target data set

- Ran a dark matter technical analysis meeting

- Updated beamline simulations to accurately predict neutrino-flux for beam off-target running

- Measured various beam position monitor's offsets to target for better understanding of the beam profile

- Developed fitter that combines on-target and off-target data sets to limit affects of systematic errors

- Improved MiniBooNE's confidence limit algorithm to include uncertainty on expected limit

- Added new cut to reduce the amount of beam uncorrelated background in data sample

\section{COHERENT}

\section{Indiana University/Oakridge National Laboratory}

Sept 2015 - Present

The COHERENT is a collaboration of multiple types of detectors searching for neutrino-nucleon coherent elastic scattering. My efforts are focused on the liquid argon single phase detector (CENNS-10). Technical Accomplishments include:

- Consultant on SciBath's neutron background measurement

- Assembling and disassembling the detector

- Development of remote shift procedure

- Consultant on analysis and simulation development

\section{SciBath}

Indiana University, Bloomington IN

Sept 2011 - Present

SciBath is a novel, large-volume, liquid scintillator tracking detector for neutrino and neutron measurements. It is a prototype for the larger FINeSSE neutrino tracking detector, and it is servicing rare-searches with stringent neutron background mitigation requirements. Technical Accomplishments include:

○ Development of waveform fitting algorithm

- Development of run control graphical interface

- Development of event display

- Development of new codding framework

- Liquid scintillator test to understand how different combinations of PPO, pseudocumene, and mineral oil affect light levels

- Development of fiber-to-fiber calibrations

- Updated simulations

\section{Previous Research Activities}

NIFFTE

Los Alamos National Laboratory, Los Alamos, NM

May 2008 - May 2013 
The Neutron Induced Fission Fragment Tracking Experiment's (NIFFTE) goal is to measure the cross section of neutron induced fission to within one percent using a Time Projection Chamber (TPC) for multiple isotopes. Technical Accomplishments include:

- Implemented active detector of the TPC into GEANT4

- Wrote detector response simulation in stand alone $\mathrm{C}++$ code

- Find and setup a thermistor readout system to measure the temperature inside the TPC chamber and integrated it with the MIDAS data acquisition system

- Participated in integrating the IOTech Slow Control hardware to read out thermocouples for measuring room temperature with MIDAS

- Participated in disassembling and assembling the detector

- Taught the NIFFTE code to three incoming graduate students

\section{Teaching Experience}

\section{Indiana University}

Assistant Instructor, Dept. Chair: Prof. Robert de Ruyter

Taught and graded two non-majors lab (total 46 students)
Bloomington, IN

Spring 2015

Abilene, IN

Aug 2007 - May 2011

Teachers Assistant, Superviosr: Prof. Rusty Towell

- Supervised intro level physics labs and graded homework (max 24 students per semester): three semesters

- Taught Astronomy Lab one night a week to twenty students: three semesters

- Tutor students in calculus and freshmen/sophomore physics: three semesters

\section{Awards}

Fall 2013: Indiana University College of Arts and Science Graduate Student Travel Award

Nov 2010: Travel award from Conference Experience for Undergraduate (CEU) for Fall

Division of Nuclear Physics (DNP) Conference

Oct 2009: Travel award from CEU for Fall DNP Conference

Spring 2009: Sigma Pi Sigma Inductee

Oct 2008: Lodging award from CEU for Fall DNP Conference

Spring 2008: Phi Eta Sigma Inductee

\section{Computer skills}

- ROOT

- GEANT4

- MIDAS 

- $\mathrm{C} / \mathrm{C}++$
- Perl
- IATEX
- Python
- HTML
- PHP
- Shell
- FORTRAN
- MySQL
- JavaScript
- Office
- Linux

\section{Presentations}

Oral

Joint Experimental-Theoretical Physics ("Wine and Cheese") Seminar:

FNAL, Batavia, IL

MiniBooNE Results from the First Proton Beam-Dump Dark Matter Search

April Meeting of the American Physical Society (APS):

Baltimore, MD

Accelerator-Produced sub-GeV Dark Matter Search using MiniBooNE

\section{PhD Candidacy Seminar:}

Bloomington, IN

Accelerator-Produced Light Dark Matter Search using MiniBooNE

Fall Meeting Division of Nuclear Physics (DNP) of APS:

Newport News, VA

Oct 2013

A Measurement of Underground Cosmic Neutron Fluxes with SciBath

\section{NNSA Fission Workshop:}

Los Alamos, NM

Feb 2012

NIFFTE Simulations

\section{American Nuclear Society Summer Meeting:}

Hollywood, FL 


\section{Abilene Christian University Undergraduate Research Festival:}

Abilene, TX

Progress in Nuclear Power Safety: Overview of the NIFFTE Experiment

Fall Texas Section Meeting of APS:

San Antiano, TX

Oct 2010

Simulations of the NIFFTE High Precision TPC

\section{Abilene Christian University Undergraduate Research Festival:}

Abilene, TX

Simulations of the NIFFTE Time Projection Chamber

Fall Texas Section Meeting of APS:

San Marcas, TX

Oct 2009

Simulations of the NIFFTE Time Projection Chamber

\section{Abilene Christian University Undergraduate Research Festival:}

Abilene, TX

Apr 2009

GEANT4 Simulations of the NIFFTE TPC

Fall Texas Section Meeting of APS:

El Paso, TX

Oct 2008

GEANT4 Simulation of the NIFFTE TPC

Poster

XXXIV Physics in Collision:

Bloomington, IN

Accelerator-Produced Dark Matter Search using MiniBooNE

Fermi National Laboratory (FNAL) Users Meeting:

Batavia, IL

Jun 2014

Accelerator-Produced Dark Matter Search using MiniBooNE 
Conference Experience for Undergraduate (CEU) Fall DNP Meeting:

Sante Fe, NM

Nov 2010

Slow Controls of the NIFFTE High Precision TPC

CEU Fall DNP Meeting:

Waikoloa, HI

Oct 2009

Simulations of the NIFFTE Time Projection Chamber

Sigma Pi Sigma Quadrennial Congress:

FNAL, Batavia, IL

GEANT4 Simulation of the NIFFTE TPC

\section{CEU Fall DNP Meeting:}

Oakland, CA

Oct 2008

GEANT4 Simulations of the NIFFTE TPC

\section{Leadership Experience}

Society of Physics Students Abilene Christian University Chapter:

President May 2010 - May 2011

Representing SPS in student government and organizing events

Society of Physics Students Abilene Christian University Chapter:

Secretary/Treasure

May 2008 - May 2009

Collected dues, track of all finances, and helped organize a three-part university wide forum 